\title{
Novel Mesoporous Nano-Titania/Carbon Composite Electrodes for Electrochemical Energy Storage
}

\author{
Micah J. Sussman \\ Department of Mining and Materials Engineering \\ McGill University \\ Montreal, Quebec \\ Canada
}

August 2014

A thesis submitted to McGill University in partial fulfillment of the requirements of the degree of Doctor of Philosophy

CMicah Sussman, 2014 
This thesis is dedicated to Harold Sussman 



\section{Abstract}

The development of novel scalable nanoscale materials and fabrication techniques for high performance $\mathrm{TiO}_{2} \mathrm{Li}$-ion electrodes is investigated in this thesis. The development and study of the novel fabrication methods is approached from the stand point of a binder-free electrode, and its comparison to standard binder-based electrodes, making use of commercial $\mathrm{P} 25 \mathrm{TiO}_{2}$ product as active material. The characterization of novel titania synthesized by manipulating the parameters of an aqueous process, is performed for two phases of $\mathrm{TiO}_{2}$, namely anatase and brookite, based on comprehensive nanocrystal morphological and electrochemical property assessment. Finally all is brought together by investigating the application of the novel fabrication protocol to the aqueous synthesized anatase to engineer binderfree 3-dimensional electrodes, and studying their Li-ion intercalation performance.

The fabrication of binder-free P25 nanotitania/carbon composite electrodes is pursued via formulating pastes and controlled sintering yielding enhanced electrochemical performance in comparison to standard binder-based methods using the same active and conductive components. The new paste formulation and sintering sequence provides a significant increase in dispersion of carbon due to smaller agglomerate size resulting in enhanced inter-particle (active-conduction) mixing and packing density than standard binder-based electrodes. Cyclic voltammetry and galvanostatic charge/discharge proved that the binder-free electrodes possess improved conductivity, specific capacity, and reversibility, as compared to binderbased electrodes. Most significantly, the capacity retention of the binder-free electrode was much higher than that of the binder-based electrode at 94 and 53\%, respectively. 
Using an aqueous solution based synthesis process, 2-dimensional brookite nanoplatelets and $6 \mathrm{~nm}$ anatase nanoparticles are obtained and examined for their physical and electrochemical properties as $\mathrm{Li}$-ion host materials. Brookite, itself is particularly interesting, as it is the least understood of the nanoscale $\mathrm{TiO}_{2}$ phases, especially in the form of 2-D crystal structures that are deemed advantageous for Liion diffusion. This study provides insight into the lithiation mechanism of brookite, concluding that a solid-solution is formed upon lithiation corresponding to $\mathrm{Li}_{0.5} \mathrm{TiO}_{2}$ that is isostructural with brookite. Further, the brookite nanoplatelets are discovered to undergo unique crystal structure enhancement upon cycling that allows them to quickly relax upon delithiation in an extremely efficient ( $>99.7 \%$ Coulombic efficiency) reversible manner translating to excellent $\mathrm{Li}$-ion intercalation stability.

Anatase nanoparticle are described in terms of crystallinity and surface area. Asprepared anatase has low crystallinity $(\sim 70 \%)$, but high surface area $\left(\sim 222 \mathrm{~m}^{2} \mathrm{~g}^{-1}\right)$, and when annealed at $300{ }^{\circ} \mathrm{C}$, the crystallinity increases to $\sim 90 \%$ but the surface area drops to $120 \mathrm{~m}^{2} \mathrm{~g}^{-1}$. Electrochemically, this allows the annealed sample with higher crystallinity to increase its diffusion-based lithium insertion capability, thus giving it high capacity at low rate, and comparable capacity at high rate to the as-prepared anatase, whose high surface area allows for increased pseudocapacitive type storage. These results point to the importance of tailoring anatase's properties (crystallinity vs. surface-area) depending on the desired performance: long-term cycling life at low rate vs. high rate capability at lower capacity retention.

Finally, the novel fabrication method is combined with the aqueous synthesized anatase, and self-supporting 3-dimensional electrodes are investigated. It is demonstrated that the 3-D electrodes fabricated using the novel paste formulation and controlled sintering protocols provide a highly conducting percolating network of carbon along with inter-particle necking resulting in optimum active material/carbon/electrolyte interfacing for facile lithium insertion. Electrochemical tests show that 3-D electrodes have significantly higher capacity over their standard counterparts (180 versus $150 \mathrm{~mA} \mathrm{~h}^{-1}$ ), and also good capacity retention at high rate. The 
newly developed 3-D electrode fabrication concept can provide a fertile platform for superior Li-ion intercalation electrodes involving other than nanotitania anode and cathode materials. 



\section{Résumé}

Le développement de nouveaux matériaux à l'échelle nanométrique et des techniques de fabrication d'électrodes $\mathrm{TiO}_{2} \mathrm{Li}$-ion de hautes performances est étudiée dans cette thèse. Le développement et l'étude des méthodes de fabrication sont abordés du point de vue d'une électrode sans liant et comparés à des électrodes à base de liants standard, faisant usage du produit commerciaux $\mathrm{P} 25 \mathrm{TiO}_{2}$ comme matière active. La caractérisation de l'oxyde de titane synthétisé en manipulant les paramètres d'un procédé aqueux, est effectuée pour deux phases de $\mathrm{TiO}_{2}$, à savoir l'anatase et la brookite, la morphologique nanocristal et les propriétés électrochimiques sont évaluées. Enfin, l'application du protocole de fabrication novateur pour l'anatase synthétisé aqueuse pour concevoir des électrodes 3 dimensions sans liant est étudié, ainsi que leur performance d'intercalation Li-ion.

La fabrication des électrodes P25 nanotitania/carbone composites sans liant est continuée par la formulation de pâtes et frittage contrôlé pour un rendement amélioré de la performance électrochimique par rapport aux méthodes à base de liant standard en utilisant les mêmes composants actifs et conductrices. La nouvelle étape de formulation de la pâte et le frittage donne une augmentation significative de la dispersion du carbone due à une plus petite taille d'agglomérat résultant en un meilleur mélange inter-particules (actif-conduction) et une plus grande densité que les électrodes à base de liants classiques. La voltamétrie cyclique et galvanostatique de charge/décharge ont prouvé que les électrodes sans liant possèdent une conduction, la capacité spécifique et la réversibilité améliorées par rapport aux électrodes à base de liant. Plus important encore, la rétention de la capacité de l'électrode sans liant était beaucoup plus élevée que celle de l'électrode 
à base de liant, passant de 53 à 94\%.

En utilisant un procédé de synthèse de solution à base aqueuse, des nanoplaquettes de brookite en deux dimensions et des nanoparticules d'anatase de $6 \mathrm{~nm}$ sont obtenues et examinées pour leurs propriétés physiques et électrochimiques comme matériaux hôtes de Li-ion. La brookite est particulièrement intéressant, car elle est la phase de $\mathrm{TiO}_{2}$ la moins bien comprise à l'échelle nanométrique, notamment sous forme cristallines 2-D qui sont jugés avantageux pour la diffusion de Li-ion. Cette étude permet de mieux comprendre le mécanisme de lithiation de brookite, concluant qu'une solution solide est formé par lithiation correspondant à $\mathrm{Li}_{0,5} \mathrm{TiO}_{2}$ qui est isistructural avec la brookite. En outre, les nanoplaquettes de brookite ont subi des améliorations uniques de la structure cristalline durant les cycles ce qui leurs permettent de relaxer rapidement durant délithiation et efficacement $(>99,7$ $\%$ d'efficacité de Coulomb) et de manière réversible, ce qui donne une excellente stabilité d'intercalation Li-ion.

Anatase nanoparticules sont décrits en termes de leurs cristallinités et de leurs surfaces. L'anatase a une faible cristallinité $(\sim 70 \%)$, mais une grande surface $\left(\sim 222 \mathrm{~m}^{2} \mathrm{~g}^{-1}\right)$ et quand elle est recuit à $300^{\circ} \mathrm{C}$, la cristallinité augmente à $\sim 90 \%$, mais sa surface diminue $\left(120 \mathrm{~m}^{2} \mathrm{~g}^{-1}\right)$. électrochimiquement, ce qui permet un échantillon recuit avec une cristallinité plus élevé pour augmenter sa capacité d'insertion à base de diffusion de lithium, ce qui lui donne une grande capacité à faible taux et une capacité comparable à l'anatase à haut taux, dont la grande surface permet d'accroître la capacité de stockage. Ces résultats soulignent l'importance d'adapter les propriétés de l'anatase (cristallinité vs l'aire de surface) en fonction de la performance souhaitée : la longue vie de cyclage à taux par rapport à un taux rapide vitesse avec une capacité de rétention inférieure.

Enfin, le nouveau procédé de fabrication est combiné à la synthèse aqueuse de l'anatase et des électrodes 3 dimensions autoportantes sont étudiés. Il est démontré que les électrodes 3-D fabriqués en utilisant la nouvelle formulation de la pâte et des protocoles de frittage contrôlées donnent un réseau de carbone percolant très

viii 
conducteur avec striction entre les particules résultant en une interface matière active/carbone/électrolyte optimum pour l'insertion de lithium. Les tests électrochimiques montrent que les électrodes 3-D ont une capacité nettement plus élevé sur leurs homologues standard ( 180 contre $\left.150 \mathrm{~mA} \mathrm{~h} \mathrm{~g}^{-1}\right)$ et aussi une bonne capacité de rétention à haut taux. La notion de fabrication d'électrode 3-D nouvellement développé peut fournir une plateforme fertile pour des meilleures électrodes d'intercalation Li-ion comportant d'autres matériaux que les nanotitania pour anode et cathode. 



\section{Acknowledgements}

I would like to first thank my funding agency for providing support for this research. This work has been financially supported by a McGill Engineering Doctoral Award, jointly funded by the McGill University Faculty of Engineering and the Natural Sciences and Engineering Research Council's strategic grant program.

There are many individuals that need to be thanked for their contributions to the making of this thesis. First, and foremost, I want to thank my supervisor, Professor George P. Demopoulos (never forget the $P$ ), for everything that he has taught me. From the beginning, he pushed me to take charge and make things happen. It was with his guidance that I learned that even if I was good, I could always be better. I enjoyed every meeting, and even when we did not agree, I always felt like he trusted me to make good research decisions. He also taught me, little by little, how to strengthen my writing, and make effective discussion of my work. Overall, I always felt supported and knew that if I needed something, George was there to provide backing.

I want to thank Matthew Gallaugher and Hendrix Demers. Without friends like you, I would never have made it this far. You guys were a constant source of diversion from the stress of finishing this thesis. The barbeques, video game sessions, and even the Caribou were worth every minute and I cannot think of two people I would rather spend time with at a butcher shop. Also, I want to thank Thomas Feldmann for putting up with sitting next to me in an office for 3 years. I am sure that it was not the easiest thing, but you put up with me and my antics pretty well, and helped me get this thesis to the finish line.

I want to acknowledge my family for their support throughout this process. Even 
though sometimes I was a pain, I really appreciated the chance to rant and rave about problems that seem insignificant now that I look back on them. You guys have been there since the beginning and I know that you have been in my corner since day one. I want to especially thank my parents and step-parents for making trips to come to Montreal in January when no one else would have, just to spend time with me.

Finally, I want to thank my amazing wife, Dr. Amanda Fortier-Sussman, who understood what I was going through when others could not. She was an unwaivering support for me who understood the stress of a $\mathrm{PhD}$ and knew what it took to get one finished. She was and is my biggest fan and my best friend. I am pretty sure that without her this thesis would never have gotten finished and I would be a graduate student for the rest of my life. Also, I want to leave a message in here for my son, Jonathan Harold Sussman. Though you are not born yet, you are one of the best motivations for finishing this $\mathrm{PhD}$ that I had while I lived away from your mother. I know that you will bring us both so much joy, and even more stress, and maybe one day, you will follow in our footsteps and do a $\mathrm{PhD}$ of your own. No matter what, I am excited for you to join us on this crazy planet we call Earth. 


\section{Contributions of Author and Co-Authors of Published Works Presented in This Thesis}

Micah Sussman, Nicolas Brodusch, Raynald Gauvin, and George P. Demopoulos, Binder-Free Fabrication of Nanotitania/Carbon Lithium-ion Intercalation Electrodes. Journal of the Electrochemical Society, 165(5), A3100-3107 (2013).

Micah Sussman, Mert Celikin, Amrita Yasin, George P. Demopoulos, Mesoporous Brookite Nanoplatelets with Superior Lithium-ion Intercalation Stability. Electrochimica Acta, 138, 215-223 (2014).

Micah Sussman, Amrita Yasin, George P. Demopoulos, On the Complex Interplay of Crystallinity and Surface Area Effects on Li-Ion Intercalation and Pseudocapacitive Storage Properties of Nanocrystalline Anatase, Journal of Power Sources, 272, 58-67 (2014).

Micah Sussman, N. Brodusch, R. Gauvin, George P. Demopoulos, Engineering 3-D Li-Ion Electrodes with Enhanced Charge Storage Properties based on SolutionProcessed and Sintered Anatase Nanocrystal-Carbon Mesoporous Structures, ACS Sustainable Chemistry \& Engineering, Submitted (2014).

In all these works, the author has performed detailed analysis and characterization of the materials. All physical characterization was performed by the author, with the exception of Field Emission Scanning Electron Microscopy and Transmission Electron Microscopy, which was performed by Nicolas Brodusch and Mert 
Celikin respectively. While the operation of this equipment was performed by others, the author analyzed all data received therein. Further, all electrochemical characterization and analysis was performed by the author.

The comprehensive supervisory role was conducted by the official supervisor (Prof. George Demopoulos). The guidance and direction on issues of scanning electron microscopy were given to the author by Professor Raynald Gauvin.

In addition, the aqueous $\mathrm{TiO}_{2}$ powders were synthesized by Amrita Yasin (fellow $\mathrm{PhD}$ student). She ran the process to synthesize both the aqueous anatase and brookite nanoparticles that were studied (Electrochimica Acta and J. Power Sources).

The accuracy of the above statements is attested by the student's supervisor.

George P. Demopoulos 


\section{Contents}

Abstract iii

Acknowledgements

Contributions of Author and Co-Authors xiii

Contents $\quad$ xv

$\begin{array}{lc}\text { List of Figures } & \text { xix }\end{array}$

$\begin{array}{ll}\text { List of Tables } & \text { xxvii }\end{array}$

1 Introduction 1

1.1 Motivation .................... 1

1.2 Objective of Present Work .............. . . . 4

1.3 References.................. 5

2 Literature Review $\quad 7$

2.1 Overview ....................... 7

2.2 Lithium-Ion Batteries . . . . . . . . . . . . . . . 7

2.2.1 Introduction . . . . . . . . . . . . . 7

2.2.2 Basic Principles of Lithium-Ion Batteries . . . . . . . 8

2.2.3 Pseudocapacitance ................ 11

2.3 Titania--Physical and Electrochemical Properties . . . . . . . . 15

2.3.1 Rutile .................... 16

2.3 .2 Brookite.................. 20

2.3.3 Anatase ..................... 24

2.4 Binder-Free Electrode Fabrication . . . . . . . . . . . . 30

2.5 References.................... 34 
3 Binder-Free Fabrication of Nanotitania/Carbon Lithium-ion Intercalation Electrodes $\quad 43$

3.1 Abstract ...................... . . 43

3.2 Introduction . . . . . . . . . . . . . . . 44

3.3 Experimental ..................... 47

3.3.1 Electrode Fabrication . . . . . . . . . . . . . . . 47

3.3.2 Physical Characterization . . . . . . . . . . . . 48

3.3.3 Electrochemical Characterization ......... . 50

3.4 Results and Discussion . . . . . . . . . . . . . . . . 51

3.4.1 Powder Characterization ............ 51

3.4.2 Electrode Film Structure . . . . . . . . . . . . . 52

3.4.3 Electrochemical Performance ........... 58

3.5 Conclusions . . . . . . . . . . . . . . . . . 65

3.6 Acknowledgements ..................... 66

3.7 References . . . . . . . . . . . . . . . . 66

4 Mesoporous Brookite Nanoplatelets with Superior Lithium-ion Intercalation Stability $\quad 71$

4.1 Abstract .................... 71

4.2 Introduction . . . . . . . . . . . . . . . 72

4.3 Experimental .................. . . 76

4.3.1 Synthesis and Characterization . . . . . . . . . 76

4.3.2 Electrochemical Measurements . . . . . . . . . . . 77

4.4 Results and Discussion . . . . . . . . . . . . . . . 78

4.4.1 Material Characterization . . . . . . . . . . . 78

4.4.2 Li-Ion Intercalation Behavior . . . . . . . . . . . . . 82

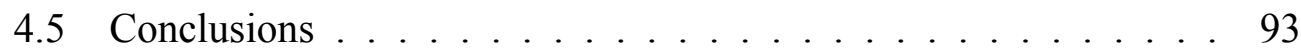

4.6 References . . . . . . . . . . . . . . . . 94

5 On the Complex Interplay of Crystallinity and Surface Area Effects on Li-Ion Intercalation and Pseudocapacitive Storage Properties of Nanocrystalline Anatase $\quad 99$

5.1 Abstract . . . . . . . . . . . . . . . . 100

5.2 Introduction . . . . . . . . . . . . . . . 100

5.3 Experimental ....................... 103

5.3.1 Synthesis and Characterization . . . . . . . . . 103

xvi 
5.3.2 Electrochemical Measurements . . . . . . . . . . . . 104

5.4 Results and Discussion . . . . . . . . . . . . . . . 105

5.4.1 Nanoanatase Characterization . . . . . . . . . . . . 105

5.4.2 Investigation of Storage Mechanisms _ . . . . . . . 110

5.5 Conclusions . . . . . . . . . . . . . . . . 125

5.6 References . . . . . . . . . . . . . . . . . . . . 126

6 Engineering 3-D Li-Ion Electrodes with Enhanced Charge Storage Properties based on Solution-Processed and Sintered Anatase NanocrystalCarbon Mesoporous Structures 131

6.1 Abstract . . . . . . . . . . . . . . . . . 132

6.2 3-D Anatase Electrodes . . . . . . . . . . . . . . . . . 132

6.3 Acknowledgements . . . . . . . . . . . . . . . . . 141

6.4 References . . . . . . . . . . . . . . . . . . . . 141

7 Synopsis 145

7.1 Global Conclusions . . . . . . . . . . . . . . . . . . 145

7.2 Claims to Originality . . . . . . . . . . . . . . . . . . . . 149

7.3 Future Work . . . . . . . . . . . . . . . . . 150

A X-ray Diffraction Peak List 153

B Experimental Methods 159

B.1 Introduction . . . . . . . . . . . . . . . . . 159

B.2 Chemicals and Materials . . . . . . . . . . . . . . . 159

B.2.1 Synthesis of Active Materials . . . . . . . . . . . 159

B.2.2 Electrode Fabrication . . . . . . . . . . . . . . . . 161

B.2.3 Electrochemical Cell Assembly . . . . . . . . . . . . 162

B.3 Treatment and Fabrication . . . . . . . . . . . . . 162

B.3.1 Pre-treatment of Electrode Substrates . . . . . . . . . 162

B.3.2 Electrode Fabrication . . . . . . . . . . . . . . . . 163

B.3.3 Electrochemical Cell Fabrication . . . . . . . . . . . . 165

B.4 Physical Characterization Methods . . . . . . . . . . . . . 167

B.4.1 Brunauer-Emmett-Teller (BET) Surface Area . . . . . 167

B.4.2 X-Ray Diffraction . . . . . . . . . . . . . . . . 168

B.4.3 Scanning Electron Microscopy . . . . . . . . . . . . . 170 
B.4.4 Transmission Electron Microscopy . . . . . . . . . . 172

B.5 Electorchemical Characterization Methods . . . . . . . . . . . 172

B.5.1 Cyclic Voltammetry . . . . . . . . . . . 172

B.5.2 Galvanostatic Cycling ............. . 173

B.5.3 Electrochemical Impedance Spectroscopy . . . . . . . . . 174

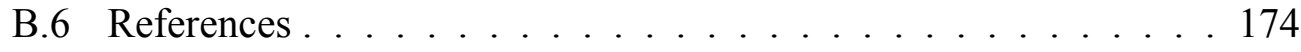

C Electrochemical Testing of Novel and Standard P25 Electrodes 177

C.1 Experimental . . . . . . . . . . . . . . . 177

C.2 Results ....................... 178

D Supplementary Information for Mesoporous Brookite Nanoplatelets with Superior Lithium Intercalation Stability 181

D.1 References . . . . . . . . . . . . . . . . . . 182

E Supplementary Information for On the Complex Interplay of Crystallinity and Surface Area on Li-Ion Diffusion and Pseudocapacitive Storage Properties of Nanocrystalline Anatase

F Supplementary Information for Engineering 3-D Li-Ion Electrodes with Enhanced Charge Storage Properties based on Solution-Processed and Sintered Anatase Nanocrystal-Carbon Mesoporous Structures 211

F.1 Supplementary Characterization Data . . . . . . . . . . . 211

F.2 Sintering Effects . . . . . . . . . . . . . . . . . 219

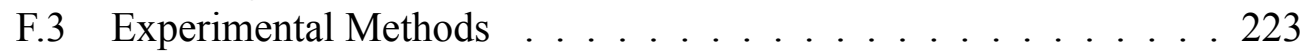

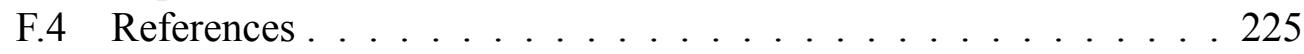




\section{List of Figures}

1.1 Breakdown of greenhouse gas emissions by sector . . . . . . . . 1

1.2 Proposed changes in energy production by percentage showing increases in renewable energy and the addition of carbon capture and sequestration (CCS) for gas and coal plants . . . . . . . . 2

1.3 Hybrid car with battery/supercapacitor pair. The graph qualitatively shows the initial power requirements for starting from a dead stop 3

2.1 Schematic of delithiation of active material in positive electrode ${ }^{1}$. 10

2.2 Schematic of the electric double layer . . . . . . . . . . 12

2.3 Galvanostatic charge/discharge curves representing materials with (a) diffusion control and (b) pseudocapacitance . . . . . . . . . 14

2.4 Ideal rutile crystal structure showing vacancy channels. . . . . . 17

2.5 Discharge (lithiation) curve for rutile $\mathrm{TiO}_{2}$ showing three distinct regions (top) and the crystallographic changes that accompany each region (bottom) . . . . . . . . . . . . . . . . . . . . . 19

2.6 Crystal structure of brookite displaying $\mathrm{TiO}_{6}$ octahedra $\ldots \ldots .21$

2.7 Brookite lattice highlighting the lithium environment . . . . . . 23

2.8 Anatase crystal structure represented by (a) $\mathrm{TiO}_{6}$ polyhedra and (b) planar $\mathrm{Ti}_{3} \mathrm{O}$ building blocks . . . . . . . . . . . . . . 25

2.9 Break down of voltage profile depicting theoretical phase transitions of anatase (a) and experimental phase and mechanism changes during lithiation (b). The hump (circled in red) in the experimental profile shows that the phase change from $\beta$ - $\mathrm{Li}_{\mathrm{x}} \mathrm{TiO}_{2}$ to $\gamma-\mathrm{Li}_{1} \mathrm{TiO}_{2}$ does not occur over a long enough period of time to significantly impact the storage capacity. . . . . . . . . . . . . . . . 27

2.10 Schematic drawing of intercalation and deintercalation processes . 28

3.1 Flowchart of novel electrode fabrication method. . . . . . . . . 49

3.2 STEM-BF and SE images with t-EFSD patterns for anatase (left) and rutile (right) phase particles. . . . . . . . . . . . . . 53 
3.3 Electron micrograph of P25 powder at 200,000x magnification with rutile and anatase particle varieties labelled. . . . . . . 53

3.4 Scanning electron micrographs of the two electrodes at different magnifications: Standard method electrode at 500x (a) and 4000x (b); novel method electrode (7.5 wt\% C) at 500x (c) and 4000x (d). 55

3.5 STEM-BF images of 70 to $90 \mathrm{~nm}$ thick electrode slices of the standard (a), $7.5 \mathrm{wt} \%$ carbon novel (b), and $15 \mathrm{wt} \%$ carbon novel method electrodes $(\mathrm{c}) \ldots \ldots \ldots$. . . . . . . . . . . 56

3.6 Comparison of carbon agglomerate size distribution normalized to percentage of total carbon agglomerates for the two electrodes. ${ }^{2}$. 57

3.7 High magnification scanning electron micrographs of the standard (a), $7.5 \mathrm{wt} \%$ carbon novel (b), and $15 \mathrm{wt} \%$ carbon novel method electrodes (c) . . . . . . . . . . . . . . . . . 59

3.8 Reproducible (second to fifth cycles) cyclic voltammograms for electrodes fabricated using the standard, $7.5 \mathrm{wt} \%$ carbon novel, and $15 \mathrm{wt} \%$ carbon novel methods (a). Differences between the first and fifth cycle CVs for one of the electrodes $(7.5 \mathrm{wt} \%$ carbon novel method) (b) ${ }^{3} \ldots \ldots \ldots \ldots$. . . . . . . . 60

3.9 Figure 9. Galvanostatic (second cycle) discharge (lithiation) (a) and charge (delithiation) (b) curves for electrodes fabricated using the standard, $7.5 \mathrm{wt} \%$ carbon novel, and $15 \mathrm{wt} \%$ carbon novel methods. . . . . . . . . . . . . . . 62

3.10 Galvanostatic cycling of the standard and novel ( $7.5 \mathrm{wt} \%$ carbon) electrodes: Coulombic efficiencies (a) and charge/discharge capacities $(b){ }^{4} \ldots \ldots \ldots \ldots \ldots$

4.1 X-ray diffraction pattern for aqueous synthesized brookite material. 79

4.2 Transmission electron micrographs of brookite nanoplatelets. Low magnification of aggregated nanoplatelets (a), high magnification of large aggregated nanoplatelets (b) and high magnification of a small cluster of nanoplatelets (c). SAED of (d) Low magnification aggregate (a) and (e) high magnification clustered platelets (c). . . 80 
4.3 Voltage profiles of the 2nd cycle lithiation and delithiation of brookite (solid line) and P25 anatase (dashed line). The capacities of the brookite and P25 are based on the total amount of $\mathrm{TiO}_{2}$ in the electrode. This amount includes the rutile component, which does not lithiate. . . . . . . . . . . . . . . . . .

4.4 Voltage profile of first lithiation of brookite nanoplatelets over 36 hours (a), Representative schematic of Nyquist plot during lithiation (b), trends in charge transfer resistance $R_{c t}$ (c) and double layer capacitance $C_{d l}(\mathrm{~d})$ during the voltage drop from $3 \mathrm{~V}$ to $1 \mathrm{~V}^{5}$. . . 86

4.5 Diffraction patterns for pristine electrode (I), electrode after first lithation (II), electrode after first delithiation (III), and electrode after 10 cycles (IV). Amorphous humps caused by glass slide is marked with a "*" while the peaks for PVDF is indicated with an enclosure. ..................... 89

4.6 2nd cycle lithiation and delithiation profiles of the brookite nanoplatelets at various rates (top) and the lithiation (discharge) capacity retention of the brookite component over multiple cycles at various rates (bottom). The capacity values reflect the mass of active brookite only, not the total mass that comprises brookite and inactive rutile.

4.7 Schematic of nanoplatelet (left) and nanoparticle (right) after lithiation over a finite period of time. . . . . . . . . . . . . . . . 93

5.1 X-ray diffraction patterns for as-prepared anatase (A0; I), and anatase samples annealed at $200^{\circ} \mathrm{C}$ (A200; II), $300^{\circ} \mathrm{C}$ (A300; III),

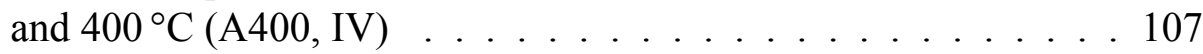

5.2 Transmission electron micrographs of A0 (a), A200 (b), A300 (c), and A400 (d) anatase samples. . . . . . . . . . . . . . . 109

5.3 Specific capacity and coulombic efficiency data for all samples cycled 100 times at $10 \mathrm{C}$ rate $\left(\mathrm{C}=168 \mathrm{~mA} \mathrm{~h}^{-1}\right)$ between 3 and 1 $\mathrm{V}^{6} \ldots \ldots \ldots \ldots \ldots \ldots \ldots$

5.4 Rate capability data for A0 and A300 showing the reversible capacity at $1 \mathrm{C}, 2 \mathrm{C}, 5 \mathrm{C}, 10 \mathrm{C}, 20 \mathrm{C}$ and back at $1 \mathrm{C}$ (a) and the corresponding voltage profiles for A0 (b) and A300 (c). . . . . . . . . 114

5.5 Nyquist plots for A0 (a) and A300 (b) at 0, 25, 50, 75, and 100\% lithiation. Zoomed in view of high frequency impedance for A0 (c) and A300 (d). ${ }^{7} \ldots \ldots \ldots$. . . . . . . . . . . . 116 
5.6 Differential capacity (DQ/DV) plots of A0 (a) and A300 (b) at 1C, 2C, 5C, 10C, and 20C. Comparison of DQ/DV of A0 and A300 at $1 \mathrm{C}(\mathrm{c})$ and $10 \mathrm{C}(\mathrm{d})$ rates. . . . . . . . . . . . . . . . 122

5.7 Specific capacity and coulombic efficiency for A0 and A300 lithiated at $10 \mathrm{C}$ rate $\left(1 \mathrm{C}=168 \mathrm{~mA} \mathrm{~g}^{-1}\right)$ over 500 cycles. . . . . . 123

6.1 SEM micrographs showing topographical, composition, and compositional heat maps for standard (a-c) and 3-D (d-f) electrodes. Heat maps highlight electrode macropores (white), carbon (red), $\mathrm{TiO}_{2}$ (green), and mesopores within $\mathrm{TiO}_{2}$ aggregates (blue). . . . . 137

6.2 Cross-section of 3-D electrode film displaying percolating carbon networks as a topographic micrograph (a), compositional micrograph (b), and a heat map (c) that associates the two images. . . . 138

6.3 Galvanostatic charge/discharge over 100 cycles between cutoff voltages of $1 \mathrm{~V}$ (discharge) and $3 \mathrm{~V}$ (charge) at $1 \mathrm{C}$ rate $\left(168 \mathrm{~mA} \mathrm{~g}^{-1}\right) 139$

6.4 Galvanostatic charge/discharge over 100 cycles between cutoff voltages of $1 \mathrm{~V}$ (discharge) and $3 \mathrm{~V}$ (charge) at $1 \mathrm{C}$ rate $\left(168 \mathrm{~mA} \mathrm{~g}^{-1}\right) 141$

B.1 Chemical structures of $\alpha$-terpineol (left) and ethyl cellulose (right). 160

B.2 Schematic of the continuous synthesis process. . . . . . . . 161

B.3 Time/Temperature profile for controlled sintering process . . . . . 165

B.4 Schematic of commercial three-electrode cell setup. . . . . . . . . 166

B.5 Schematic of in-house three electrode cell setup. . . . . . . . . . . 168

B.6 Schematic of Swagelok-type cell set up. . . . . . . . . . . . . 169

B.7 JCPDS X-ray diffraction patterns . . . . . . . . . . . 170

C.1 Galvanostatic charge/discharge over 100 cycles between cutoff voltages of $1 \mathrm{~V}$ (discharge) and $3 \mathrm{~V}$ (charge) at $1 \mathrm{C}$ rate $\left(168 \mathrm{~mA} \mathrm{~g}^{-1}\right) 180$

D.1 Reitveld refinement of synthesized brookite (a) and the deconvolution of the two main brookite peaks (b). The individual peak convolutions are below the observed and refined patterns, and the gray pattern below the $\mathrm{x}$-axis is the refinement error. . . . . . . 183

D.2 The $\mathrm{N}_{2}$ isotherm for aqueous synthesized brookite . . . . . . . . . 184

D.3 Voltage profiles for the lithiation at various cycles (1-4, 30 and 50 ) at $\mathrm{C} / 10$ rate of the as synthesized brookite. The capacity is calculated based on the total mass of $\mathrm{TiO}_{2} \ldots \ldots \ldots$. . . . . 185 
D.4 Nyquist plots of brookite at various levels of lithiation during the formation step---refer to Figure 4.4 in the published paper. During the initial voltage drop from $3.0 \mathrm{~V}$ to $2.0 \mathrm{~V}$ (a) the diffusion tail length decreases. From $1.9 \mathrm{~V}$ to $1.725 \mathrm{~V}$ (b) the diffusion tail length remains constant. As the voltage $1.7 \mathrm{~V}$ to $1.6 \mathrm{~V}$ (c) the charge-transfer resistance increases while everything else remains constant. Finally as the voltage drops from $1.5 \mathrm{~V}$ to the cutoff voltage at $1 \mathrm{~V}(\mathrm{~d})$, the diffusion tail and charge-transfer resistance increase. . . . . . . . . . . . . . . . . . . . . . 189

D.5 Plot of $Z_{W}$ phase angle versus voltage. . . . . . . . . . . . 190

D.6 Equivalent circuit for both pristine and brookite cycled 10 times (a) and equivalent circuit for brookite during lithiation (b). $R_{e}$ is the electrolyte resistance, $R_{c} s$ is the charge separation resistance, $C_{c} s$ is the charge separation capacitance (the combination of which gives the charge separation overpotential), $R_{c t}$ is the chrage transfer resistance, $C_{d l}$ is the double layer capacitance and $Z_{W}$ is the Warburg impedance. . . . . . . . . . . . . . . . . . . 192

D.7 Annotated Nyquist plots for pristine brookite (a) and delithiated brookite after 10 cycles (b). Re is the electrolyte resistance, $C_{c s}$ is the charge-separation capacitance, $R_{c s}$ is the charge-separation resistance, $C_{d l}$ is the double layer capacitance, and $R_{c t}$ is the charge-

D.8 Zoomed in XRD patterns of pristine (I), lithiated (II) and delithiated(III) electrodes showing peak shift and loss of 121 peak of brookite upon lithiation. The amorphous hump (indicated by "*") is caused by mounting the samples to glass slides. . . . . . . . . . 197

D.9 Higher resolution version of Figure 4.4c, charge transfer resistance as a function of voltage. . . . . . . . . . . . . . . . . . . . . 198

D.10 Higher resolution version of Figure $4.4 \mathrm{~d}$, double layer capacitance as a function of voltage. . . . . . . . . . . . . . . . . . . 198

E.1 Individual X-ray diffraction patterns for A0 (a), A200 (b), A300 (c) and A400 (d). The patterns show the decrease in amorphous hump due to increased crystallinity. . . . . . . . . . . . 201

E.2 Specific capacity and coulombic efficiency data for as-synthesized anatase (A0) cycled 100 times at $1 \mathrm{C}$ rate $\left(\mathrm{C}=168 \mathrm{~mA} \mathrm{~g}^{-1}\right) \ldots$. . 202 
E.3 Plot of reversible capacity difference between A0 and A300 at various rates. At $\sim 4 \mathrm{C}$ the capacity should be the same indicating a balance between diffusion-based and surface area based as the major charge storage method. . . . . . . . . . . . . . 203

E.4 Equivalent circuit model for fitting the electrochemical impedance spectroscopy data. $R_{e}$ is the electrolyte resistance, $R_{1}$ and $C_{1}$ form a parallel element representative of the electrode surface roughness and the inhomogeneity of the lithiation of the active material. $R_{c t}$ is the charge-transfer resistance, $C_{d l}$ is the double layer capacitance and $Z_{W}$ is the Warburg impedance. $R_{c t}, C_{d l}$ and $Z_{W}$ form a Randall's circuit that explains the direct interaction between lithium ions and the active material. . . . . . . . . . . . 204

E.5 Plots showing the trend of $R_{e}$ (a), $C_{1}$ (b), $R_{1}$ (c), $R_{c t}(\mathrm{~d}), C_{d l}$ (e), and phase angle (f) at various points during lithiation. . . . . . . 205

E.6 Plots of OCV vs. $x$ in $\mathrm{Li}_{\mathrm{x}} \mathrm{TiO}_{2}$ used to calculate $d E / d x$ (a) and Z" vs $\omega^{-1 / 2}$ at various points during the lithathiation of $\mathrm{A} 0$ (b) and A300 (c) used to determine the pre-exponential factor, $A$, used in the calaculation of $D_{L} i \ldots \ldots$. . . . . . . . . . . . 206

E.7 Plot comparing the diffusion coefficients of $\mathrm{A} 0$ and $\mathrm{A} 300$ at various lithiation states. . . . . . . . . . . . . . . 207

E.8 Differential voltage profiles for A0 (a) and A300 (b) highlighting the changes in profile shape between the 1st and 500th cycle. . . . 208

E.9 XRD patterns for A0 (a) and A300 (b) comparing the pristine electrode and the electrode after 500 cycles at 10C. Peak shift is marked by lines through the pattern for the electrode after 500 cycles. 209

E.10 Specific capacity and coulombic efficiency data for as-synthesized anatase (A0) cycled 500 times at $2 \mathrm{C}$ rate $\left(\mathrm{C}=168 \mathrm{~mA} \mathrm{~g}^{-1}\right) \ldots$. . 210

F.1 Transmission electron micrograph of the as-prepared anatase crystals. Micrograph shows a non-porous nanospherical morphology, and an average crystallite size of approximately $6 \mathrm{~nm}$. . . . . . 213

F.2 Pore size distribution for standard and 3-D electrode films. . . . . . 214

F.3 $\mathrm{N}_{2}$ Isotherms for anatase nanocrystallites, standard electrode, and 3-D electrode films. . . . . . . . . . . . . . . . . . 215 
F.4 High magnification secondary electron micrograph of the 3-D electrode structure (top) coupled with schematics of the standard and 3-D electrode structures to highlight differences. Standard electrodes have individual nanoparticles (purple) forming a larger secondary particle (blue). These secondary particles contact each other and large carbon clusters (black) at single point and are held together by PVDF binder (yellow). The 3-D electrodes have nanoparticles that neck to form a rod-like structure which builds the secondary particles. These particles also neck to each other and contact well dispersed carbon particles at multiple places within the structure. . . . . . . . . . . . . . . . . 217

F.5 Voltage-capacity profiles for standard (solid line) and 3-D (dashed line) electrodes during the initial lithiation and delithiation cycle at $\mathrm{C} / 5$ rate . . . . . . . . . . . . . . . . . . 218

F.6 Galvanostatic charge/discharge over 100 cycles between cutoff voltages of $1 \mathrm{~V}$ (discharge) and $3 \mathrm{~V}$ (charge) at $10 \mathrm{C}$ rate $\left(1680 \mathrm{~mA} \mathrm{~g}^{-1}\right) 218$

F.7 Sintering profile for 3-D electrodes with temperature steps highlighted. Temperature returns to $25^{\circ} \mathrm{C}$ after 7 hours (not shown on profile) ........................ 220

F.8 Secondary sintering profile with lower necking temperature. Temperature is held for longer period of time before returning back to

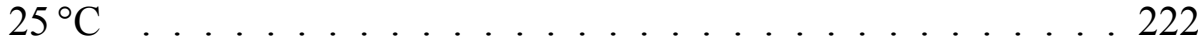

F.9 Rate capability for 3D and 3D400 cycled between upper and lower cutoff voltages of 3 and $1 \mathrm{~V}$, respectively . . . . . . . . . . 222 



\section{List of Tables}

2.1 Physical and electrochemical data for rutile nanotitania anode materials. . . . . . . . . . . . . . . . . 20

2.2 Physical properties and electrochemical cycling data for various types of anatase $\mathrm{TiO}_{2}$ morphologies $\ldots \ldots \ldots . \ldots . \ldots 31$

5.1 Key physical characterization values for the different anatase nanomaterials. . . . . . . . . . . . . . . . . . 106

A.1 JCPDS crystal phase parameters of anatase (\#00-021-1272) . . . 154

A.2 JCPDS crystal phase parameters of rutile (\#00-021-1276) . . . 155

A.3 JCPDS crystal phase parameters of brookite (\#00-016-0617) $\ldots 156$

A.4 JCPDS crystal phase parameters of $\mathrm{Li}_{0.5} \mathrm{TiO}_{2}(\# 00-038-0269) \ldots 157$

D.1 Important parameters associated with EIS over the formation cycle of brookite nanoplatelets. . . . . . . . . . . . . . . . . . 191

D.2 Capacities (based on the mNP brookite fraction only) associated with the charge and discharge as well as the coulombic efficiency for brookite at $\mathrm{C} / 10,1 \mathrm{C}$, and $10 \mathrm{C}$ rate . . . . . . . . . . . 194

E.1 Table of the important values at each point during the lithation for $\mathrm{A} 0$ and $\mathrm{A} 300$, determined by fitting the equivalent circuit to the Nyquist plots. . . . . . . . . . . . . . . . . . . . . 202

E.2 Summary of diffusion coefficient parameters. . . . . . . . . 203

F.1 Key properties of active material and electrode films based on BET surface area analysis. . . . . . . . . . . . . . . . . . . 219 


\section{Chapter 1}

\section{Introduction}

\subsection{Motivation}

In 1997, the Kyoto Protocol was adopted in an effort to reduce anthropogenic interference with the global climate system [1]. The goal of the Protocol is meant to reduce $\mathrm{CO}_{2}$ emissions, which, as seen in Figure 1.1, come mostly from the energy sector and from automobiles [2].

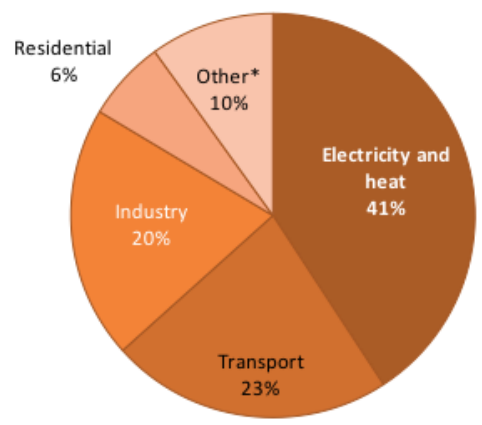

\footnotetext{
* Other includes commercial/public services, agriculture/forestry, fishing, energy industries other than electricity and heat generation, and other emissions not specified elsewhere.
}

Figure 1.1: Breakdown of greenhouse gas emissions by sector [2]

The energy sector itself has started revising its outlook on carbon emissions on its own. Starting in 2007, the Electric Power Research Institute (EPRI) performed analyses that created a technically and economically feasible roadmap for 
the electricity sector to reduce greenhouse gas emissions [3]. The goal was to drop emissions below the levels of 1990. This analysis was updated in 2009 and again in 2012, and more accurately reflects the technological changes that have potential to affect the reduction of emissions [3, 4]. The updated analysis showed the possibility of a $62 \%$ reduction in green house gasses by 2030, based on the Energy Information Agency (EIA) reference case distributed by the United States Department of Energy [5]. As seen in Figure 1.2, replacement of major producers of greenhouse gasses, mainly coal, natural gas, and petroleum, by renewable and nuclear energy production will reduce the greenhouse gas levels significantly.

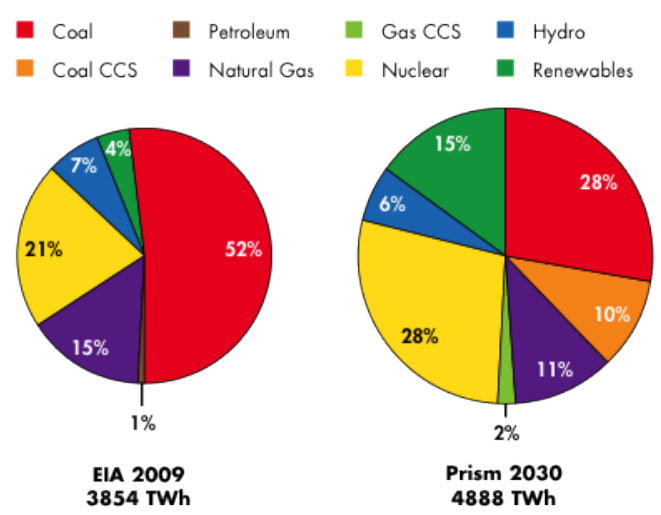

Figure 1.2: Proposed changes in energy production by percentage showing increases in renewable energy and the addition of carbon capture and sequestration (CCS) for gas and coal plants [5]

While most of the changes to the current energy sector are based on standard technology, namely the addition of nuclear plants, newly developed renewable energies pose a major problem for full integration, especially when it comes from wind and Solar (mostly in the form of photovoltaic cells) [6--8]. With reference to solar, for example, electricity is generated during the day, and peak energy usage occurs in the evening, so energy needs to be aptly stored. This need for energy storage causes an increased demand for battery systems that can give variable power and energy according to demand. 
Even though the energy sector is using batteries for larger scale energy storage, batteries are starting to make inroads into the automotive industry as it pushes to reduce emissions and expand gas mileage. With the increase in plug-in hybrid electric vehicles (PHEVs), the demand for batteries increases as well [9]. The automotive industry has started to move toward lithium ion batteries (LIBs) in cars, due to their increased Coulombic efficiency at faster discharge rates when compared to NiMH and NiCd batteries [10]. However, the current LIBs are limited by their ability to charge and discharge and cost of fabrication. In order to meet the requirements of these hybrid vehicles, supercapacitors (SC) are also being integrated, as they provide high power at extremely fast charge and discharge rates [11--15]. SCs, in this case, are used for the initial acceleration of the vehicle. Since they provide high power for a short period of time, they are drained quickly, getting the vehicle moving, before the power source changes to batteries. The batteries are then able to power the vehicle at a lower level, as seen in Figure 1.3.

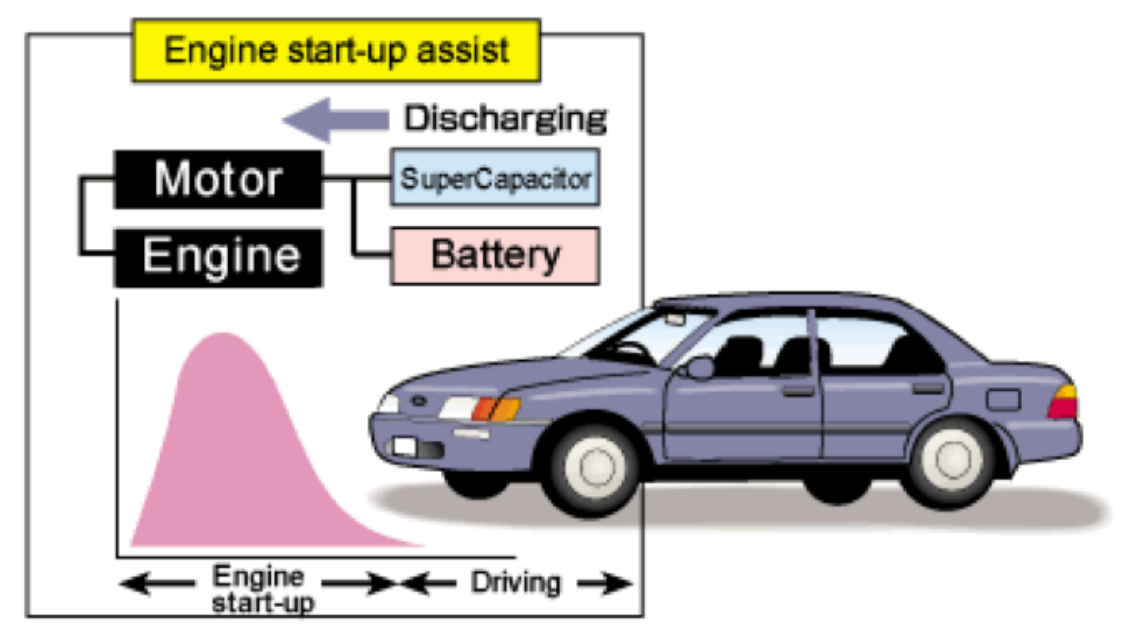

Figure 1.3: Hybrid car with battery/supercapacitor pair. The graph qualitatively shows the initial power requirements for starting from a dead stop [16] 
In this context, there is a tremendous research interest and activity in enhancing electrode performance and reducing cost by manipulating and optimizing their material properties and fabrication processes. This is true for both batteries and supercapacitors. Nanoscale $\mathrm{TiO}_{2}$ has been demonstrated to hold interesting Li-ion storage properties that can potentially find application in battery, supercapacitor, or hybrid systems $[17,18]$. Based on the aformentioned background, this thesis focuses on studying the electrochemical properties of different $\mathrm{TiO}_{2}$ nanophases as Li-ion host materials and in developing novel electrode fabrication processes for enhanced performance and applicability.

\subsection{Objective of Present Work}

In this work, nanoscale titania (anatase and brookite) electrodes have been studied via comprehensive physical characterization and electrochemical analysis to establish key material property-processing-performance relationships that can ultimately lead to fabrication of electrodes with enhanced Li-ion storage properties. Physical characterization using X-ray Diffraction (XRD) and Rietveld refinement, Scanning Electron Microscopy (SEM), Scanning Tunneling Electron Microscopy (STEM), Brunauer-Emmett-Teller (BET) surface area analysis focused on the structure and composition of the titania active material and the electrode film produced. Electrochemical characterization focused on the intercalation behavior of lithium ions into the titania lattice structure. Cyclic Voltammetry (CV), galvanostatic charge/discharge testing, rate capability, and Electrochemical Impedence Spectroscopy (EIS) were used to characterize the electrochemical properties of the titania electrodes.

The thesis is divided into seven chapters, followed by appendices providing supporting information and important external data. Following this introduction, the literature review of lithium-ion batteries and titania is described in Chapter 2. Chapter 3 describes the development of a novel binder-free method of fabricating electrodes, discussing the physical and electrochemical differences as compared 
to the standard binder-based method using Evonik P25 commercial nanotitania as active material and Fluorine-Tin-Oxide coated glass as substrate. Chapters 4 and 5 focus on evaluating the Li-ion storage properties of newly synthesized brookite and anatase titania phases and probing the effects of nanocrystal shape, crystallinity, and surface area on performance via a series of electrochemical and physical characterization tools. Chapter 6 investigates the adaptation of the binder-free fabrication method for use with the aqueous synthesized anatase titania to produce 3-Dimensional electrodes with percolating carbon networks. Lastly, Chapter 8 summarizes the major findings-conclusions of the whole research work.

\subsection{References}

[1] UNFCCC, Kyoto Protocol to the United Nations Framework, Conference of the Parties to the Climate Change Convention (1998).

[2] International Energy Agency, $\mathrm{CO}_{2}$ Emissions from Fuel Combustion Highlights, Conference of the Parties to the Climate Change Convention (2011).

[3] Electric Power Research Institute (EPRI), PRISM/MERGE Analyses 2009 Update (2009).

[4] Electric Power Research Institute (EPRI), PRISM/MERGE Analyses 2.0 (2012).

[5] Energy Information Administration, An Updated Annual Energy Outlook 2009 Reference Case Reflecting Provisions of the American Recovery and Reinvestment Act and Recent Changes in the Economic Outlook, EIA Tech Report (2009).

[6] S. Heier, Grid Integration of Wind Energy Conversion Systems, p. 426, Wiley, Hoboken, New Jersey (1998).

[7] J. M. Carrasco, L. Franquelo, J. T. Bialasiewicz, E. Galvan, R. C. Portillo, M. Angeles, M. PRats, J. I. Leon, N. Moreno-Alfonso, IEEE T Ind. Electron., 53, 1002 (2006). 
[8] S. Guha, Does Your Roof Provide Your Electrical Needs?- the Growth Prospect of Building-Integrated Photovoltaics, IEEE Photovoltaic Specialists Conference (2005).

[9] S. Davis, U.S. Demand for Batteries To Reach \$16.8 Billion in 2012 (2009).

[10] L. O. Valoen and M. I. Shoesmith, The Effect of PHEV and HEV Duty Cycles on Battery and Battery Pack Performance, E-One Moli Energy (Canada) Ltd. Technical Report (2007).

[11] A. Du Pasquier, I. Plitz, S. Menocal, G. Amatucci, J. Power Sources, 115, $171(2003)$.

[12] M. Winter and R. J. Brodd, Chem. Rev., 104, 4245 (2004).

[13] M. S. Halper and J. C. Ellenbogen, Supercapacitors: A Brief Overview, MITRE Nanosystems Group (2006).

[14] M. Jayalakshmi and K. Balasubramanian, Int. J. Electrochem. Sci., 3, 1196 (2008)

[15] C. Arbizzani, M. Mastragostino, L. Meneghello, R. Paraventi, Adv. Mater, 4, 1327 (2011).

[16] NEC/Tokin, Support Intensive Use of Automobile Energy (2004).

[17] T. Brezesinski, J. Wang, J. Polleux, B. Dunn, S. H. Tolbert, J. Am. Chem. Soc., 131, 1802 (2009).

[18] J. Wang, Y. Zhou, Y. Hu, R. O’Hayre, Z. Shao, J. Mater. Sci., 48, 2733 (2013). 


\section{Chapter 2}

\section{Literature Review}

\subsection{Overview}

This chapter reviews the theoretical background necessary for understanding the research studies later described. In the first sections, the operation of lithium ion batteries is discussed in detail. The second part of this review discusses in depth various phases of titania (rutile, brookite, and anatase) and the state of Li-ion battery research for each phase. Finally, a review of the general theory of important electrochemical characterization techniques (cyclic voltammetry, galvanostatic testing techniques, and electrochemical impedance spectroscopy) and their application to lithium ion batteries.

\subsection{Lithium-Ion Batteries}

\subsubsection{Introduction}

The electrochemical cell is made from three basic components: anode (oxidation electrode), cathode (reduction electrode), and electrolyte (ionic conductor) [1]. Batteries are devices that convert chemical energy into electrical energy via oxidation and reduction of the active materials in the electrodes. There are two distinct types of batteries: primary and secondary. The primary battery is one that cannot be easily or effectively recharged, and as such are discharged once and discarded [1]. Primary batteries tend to be convenient low-cost, light-way ways to power electronic devices, and are widely used by consumers worldwide. 
Secondary batteries, which is the focus of this work, are those that can be recharged to their original state after discharge. Secondary batteries, which include Lithium Ion Batteries (LIBs), nickel metal hydride, lead-acid batteries, as well as many others, have a much wider range of applications, from laptops to hybrid cars, even grid level energy storage [2,3]. The subsequent sections will serve as an in-depth view on the function and principles of LIBs.

\subsubsection{Basic Principles of Lithium-Ion Batteries}

A more detailed history of the LIBs that is appropriate for the purposes of this review, may be found in a review by M. Stanley Whittingham [4], which starts with the birth of the technology and describes each type of LIB system in brief detail. It is important to note however, that the earliest lithium battery was made by the Sanyo corporation for its solar powered calculators [4], and that first intercalation cells were developed by researchers at Stanford University [5]. Since then, research on intercalation compounds for LIBs has been a large part of electrochemical research.

In general, when a cell is connected to a load, electrons flow from the negative electrode (anode) toward the positive electrode (cathode) through the external circuit. The negative electrode is oxidized, and the positive electrode, in receiving the electrons is reduced. Anions migrate to the positive electrode and cations migrate to the negative electrode through the electrolyte [1]. When the cell charges, the opposite occurs. While the general concepts remain the same for charge and discharge, LIBs display some unique characteristics due to the transport of $\mathrm{Li}^{+}$ions within the electrode structures, via insertion (lithiation) and extraction (de-lithiation)

processes $[1,6,7]$. Thus the operation of LIBs is more complex than the average electrochemical cell.

The positive electrode of a LIB not only must be an electron acceptor, but it must serve as the source of $\mathrm{Li}^{+}$ions [7]. The delithiation of the positive electrode (the discharge) occurs typically by phase transformation [8]. For the most common 
commercial system $\left(\mathrm{LiCoO}_{2}\right)$, half of the lithium is removed from the positive electrode material and transported to the negative electrode, leaving $\mathrm{Li}_{0.5} \mathrm{CoO}_{2}$ [4]. While this phase transformation is stable, it leaves the cell vulnerable to combustion due to, and reactions with, the electrolyte. These phase changes affect the crystal structure, and can cause low cycle life. However, stable structures, such as $\mathrm{LiFePO}_{4}$, can be fully delithiated, and then re-lithiated over many cycles [9]. This is due to the fact that $\mathrm{PO}_{4}$ does not release an oxygen.

With the change in phase, $\mathrm{a} \mathrm{Li}^{+}$ion and an electron are released. The ion enters the electrolyte where it migrates to the other electrode, while the electron enters the electrode after traveling through the external circuit. However, the ions do not just migrate directly from the electrodes to electrolyte. The ions must diffuse from the solid-state electrode material to the electrode/electrolyte interface. Once they reach that interface, charge transfer occurs between the electrode and the electrolyte allowing the ions to cross the interface. Figure 2.1 shows a schematic of these processes.

\subsubsection{LIBs and the Importance of Nanosizing}

The rate-limiting step in the delithiation and lithiation of an intercalation compound, is the diffusion of ions within a particle $[10,11]$. Currently, commercial LIBs use micron size active material particles in their electrodes [11]. This has to do with the ease of synthesis at the commercial level. Many of the synthesis techniques currently available for nanosized particles are not feasible for commercial use due to high cost. There has previously been work to develop aqueous synthesized nanoparticles for dye-sensitized solar cells that could have application in with battery technology [12]. Other nanosizing has been attempted with $\mathrm{LiFePO}_{4}$ [9], and has been commercialized by companies such as A123 Systems and HydroQuebec.

Nanosizing is very beneficial for the electrochemical properties of the battery. By shrinking the particles, there is a large increase in surface area (from about 


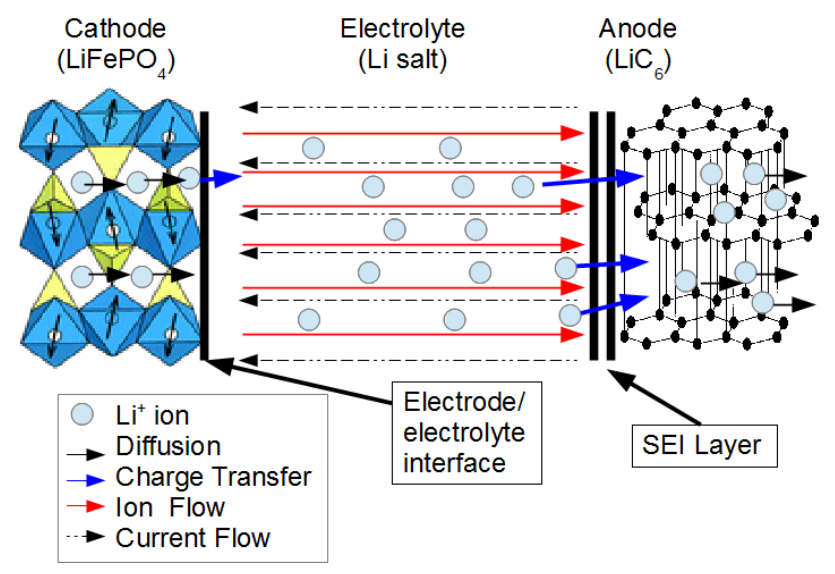

Figure 2.1: Schematic of delithiation of active material in positive electrode ${ }^{1}$

$10 \mathrm{~m}^{2} \mathrm{~g}^{-1}$ to $\left.\sim 300 \mathrm{~m}^{2} \mathrm{~g}^{-1}[11,13]\right)$. This increase in the surface area allows for a higher specific capacity owing to a larger electrode/electrolyte contact area. This is currently so because shorter diffusion paths allow full lithiation to occur within shorter time periods, as well as surface lithiation. Surface lithatiation (pseudocapacitance) depends on the surface area and not diffusion time, leading to more lithium that can be cycled at higher rate [11]. Furthermore, large surface area also provides a great reduction to the specific current density of the active material, which effectively stabilizes the electrode to preserve high capacity at higher charge/discharge current densities [14].

By shrinking the size of the particle, the ion and electron the relative transport distances are decreased. For the same length of time, an ion that enters a $6 \mathrm{~nm}$ particle will travel the same distance as one that enters a $20 \mathrm{~nm}$ particle, but the fraction of the particle size that the ion has diffused through will be greater for the smaller particle than the larger one. This means that in a smaller particle, for the same period of time, a larger fraction of a particle can store lithium, causing a

${ }^{1}$ Current does not flow through the electrolyte but rather through the external circuit. It is shown as a broken line to indicate that there are electrons transferred between the electrodes but that it is not by direct injection into the electrolyte. 
larger total charge storage.

Another aspect of nanosizing can be seen in olivine materials $\left(\mathrm{LiMPO}_{4}, \mathrm{M}=\mathrm{Fe}\right.$, $\mathrm{Mn}$ ), which are poor ionic conductors in the bulk material [15]. This is because these materials have a one-dimensional diffusion path along the [010] direction [16]. However, nanosized $\mathrm{LiFePO}_{4}$ has a smaller dimension along the [010] direction, and can be achieved using nanoparticles, nanorods with a long $a-$ or $c$-axis [17], or nanoplates with a [010] basal plane [15]. These structures have allowed more facile diffusion of lithium into the $\mathrm{LiFePO}_{4}$ lattice. $\mathrm{TiO}_{2}$ also shows interesting properties at the nanoscale. Jiang, et al [18] were able to achieve $234 \mathrm{~mA} \mathrm{~h} \mathrm{~g}^{-1}$ at high current density $\left(10 \mathrm{~A} \mathrm{~g}^{-1}\right)$ with $6 \mathrm{~nm}$ particles because they could store lithium both in the lattice and on the surface. By shrinking the diffusion path length, and increasing the surface area, they were able to take advantage of multiple aspects of nanosizing at once.

\subsubsection{Pseudocapacitance}

Storage of energy, as seen in batteries, occurs through Faradaic (indirect chemical energy storage; oxidation and reduction of electrodes) storage processes involving diffusion inside the electrode as discussed in Section 2.2.2.1 rather than direct storage charge as in electrochemical capacitors. In the case of capacitors energy is stored non-Faradaically [19] in the form of an electrical double layer. The negative and positive charges align themselves on opposite plates. The charge separation forms a gradient that is seen in the schematic in Figure 2.2. At the surface, the aligned negative charge attracts the positive charges in solution. Solvated ions will not be able to come in full contact with the surface forming the second layer of the double layer. This solvation also acts to shield the effect of the charge causing two distinct regions: the Helmholtz Layer and the Diffuse Double Layer [20--22].

There are some materials that store energy Faradaically, but exhibit the behavior of non-Faradaic capacitance. In 1963, Robert de Levie [23, 24] achieved 


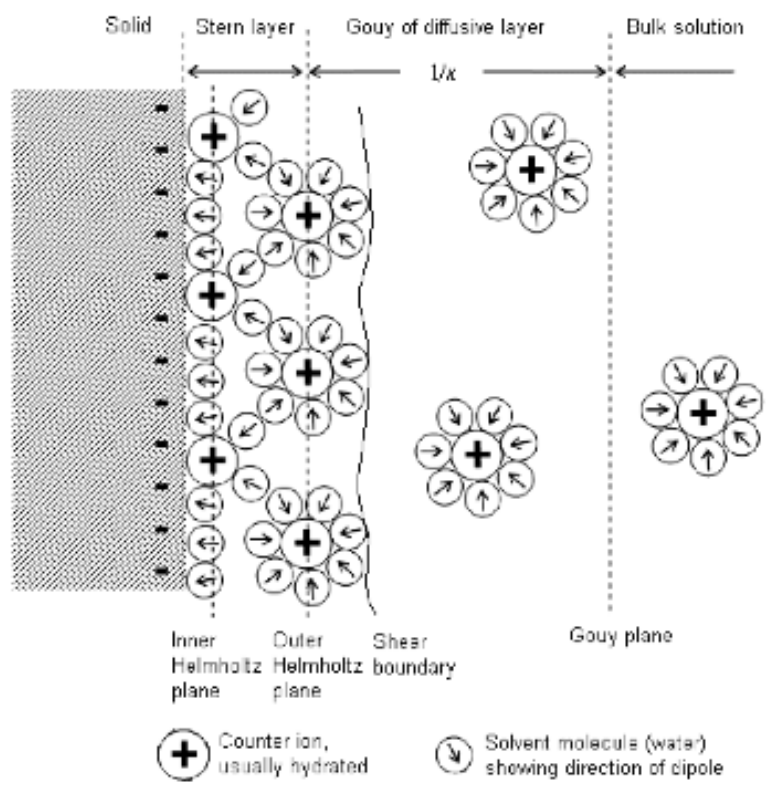

Figure 2.2: Schematic of the electric double layer [22]

capacitance using Faradaic processes, discovering what would be later called pseudocapacitance. Faradaic cells have an active material mass that is altered in direct proportionality to the quantity of electricity transferred. This is always the case for batteries, but for materials which have pseduocapacitive properties this is not always the case. Faraday's Law, which governs battery operation is as follows:

$$
m=\frac{Q M}{n F}
$$

where $m$ is the active mass in grams, $Q$ is the total charge in Coulombs, $M$ is the molar mass of the substance in grams per mole, $n$ is the valence charge and $F$ is Faraday's constant in coulombs per mole. For the case of pseudocapacitance, Faradaic charge-transfer leads to passage of extent of charge $d Q$, which, for thermodynamic reasons depend on changes in potential $d E[19,25,26]$. In this case, $d Q / d E$ is equal to a constant, $C_{\phi}$, representing the pseudocapacitance. Pseudoca- 
pacitive storage, which is different from electric double layer capacitance, presents itself either intrinsically or extrinsically [27]. Intrinsic pseudocapacity is a material property while extrinsic pseudocapacitance is usually caused by nanosizing and is not seen in bulk materials. Thus by increasing surface area, the contribution from pseudocapacitive storage is increased significantly [28]. Whether, intrinsic or extrinsic, pseudocapacitance may arise via one of three different mechanisms: adsorption, redox reactions, and ion intercalation.

With each of these mechanisms, pseudocapacitance is calculated by the same equation, varying a single term depending on the mechanism:

$$
C_{\phi}=\frac{Q F}{R T} M \bullet(1-M)
$$

Pseudocapacitance in the form of adsorption and redox reactions has been thoroughly studied and more details about it can be found in the literature [25, 29--34]. Ion intercalation, on the other hand, is the basis for pseudocapacitance seen in battery electrodes [35]. This type of intercalation storage differs from the standard intercalation as it occurs in the surface sites of the crystal structure. Though it is insertion into the crystal, it is dependent on the surface area of the particles, rather than the diffusion length. Ion intercalation, coupled with adsorption, allows some intercalation compounds to display high specific energy capacity as well as rate capability. Capacitive contributions to specific capacity are explained by the sorption of lithium into a lattice in terms of site-fraction, $X$ [36]. Intercalation is considered a three-dimensional analog to adsorption, whose pseudocapacitive contribution is based on surface coverage fraction. These contributions are given as follows:

$$
\begin{gathered}
C_{\phi}=\frac{Q F}{R T} X \bullet(1-X) \\
C_{\phi}=\frac{Q F}{R T} \theta_{B} \bullet\left(1-\theta_{B}\right)
\end{gathered}
$$


where $X$ and $\theta_{B}$ replace the general mechanism based fraction $M$. In this case, $\mathrm{X}$ is the fraction of the lattice occupied by intercalated ions, and $\theta_{B}$ is the fraction surface coverage of adsorbed ions. For pseudocapacitance within a battery, the kinetics of intercalation are not diffusion-limited, but rather are limited by surfaces processes causing the overall reaction to seem capacitive [37]. This phenomenon is rarely seen, as most intercalation compounds, including some thin films, are limited by solid state diffusion [38]. This is seen using cyclic voltammetry, where the current is proportional to $\nu^{1 / 2}$, the square root of the scan rate. With galvanostatic charge and discharge, a physical interpretation is possible, as non-diffusion controlled storage is represented by a sloped curve, while diffusion control is represented by a plateau (Figure 2.3). There are those materials that display both diffusion (true intercalation) and surface storage (pseudocapacitance) within the same curve. These materials tend to be considered pseudocapacitive under specific conditions. The non-diffusion controlled storage is assumed to be extra storage in vacant sites near the surface of the material, as is commonly seen in $\mathrm{TiO}_{2}$ electrodes [40].

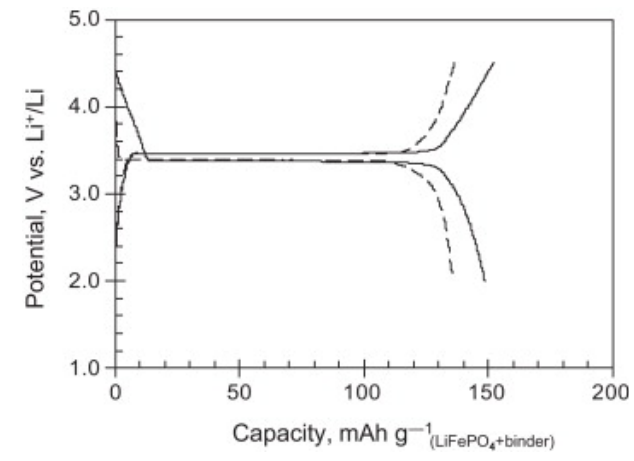

(a)

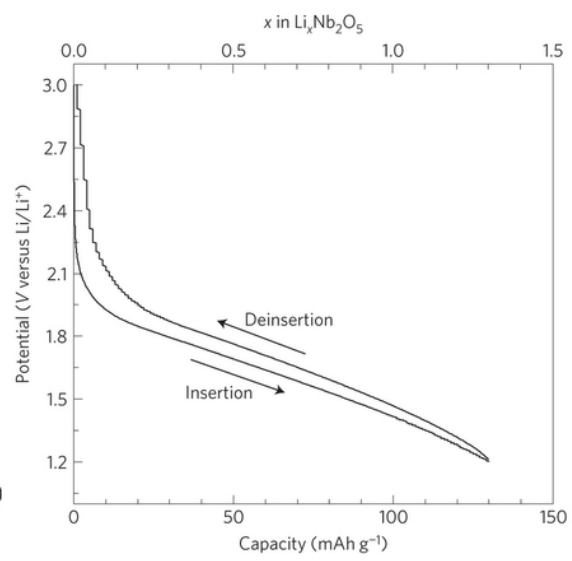

(b)

Figure 2.3: Galvanostatic charge/discharge curves representing materials with (a) diffusion control and (b) pseudocapacitance [37, 39] 


\subsection{Titania--Physical and Electrochemical Properties}

The most common material for LIB anodes is graphite [41, 42], however there are problems associated with its safety/long-life performance that is required of LIBs destined for electric vehicles arising from the formation of the well known Surface-Electrolyte Interface (SEI) layer [43--45]. This SEI layer forms as the result of side reactions with the electrolyte. It is necessary for a lithium storage in graphitic electrodes [3]. When the side reactions proliferate, and the layer becomes too thick, the cell can no longer support intercalation, and ceases to function $[3,46]$. Moreover, the formation of the SEI layer is strongly affected by the electrolyte used [41]. With different electrolyte solvents, crystallinity changes occur within the carbon, limiting its performance [47].

Graphene [48, 49], $\mathrm{SnO}_{2}$ [50], Si-based [51, 52], $\mathrm{Li}_{4} \mathrm{Ti}_{5} \mathrm{O}_{12}$ [53--55], and most recently $\mathrm{TiO}_{2}[50,56--60]$ have attracted attention as alternative to graphite anode materials. Titania, unlike many of the other graphite alternatives is unique in that can potentially find application in both LIBs (as an anode), or in supercapacitors (SCs) due to its ability to store energy via lithiation as well as via pseudocapacitive behavior at high charge and discharge rates [38, 61], and its lack of an SEI layer. Even more attractive is titania's non-toxic nature, abundance, and low cost.

The intercalation of lithium-ions into titania is described by the following reaction:

$$
T i^{I V} O_{2}+x\left(L i^{+}+e^{-}\right) \Leftrightarrow L i_{x} T i_{1-x}^{I V} T i_{x}^{I I I} O_{2}
$$

As lithium ions are discharged from the cathode, they reach the anode and enter the $\mathrm{TiO}_{2}$ lattice. They are accompanied by an electron (in order to balance the charge), and the $\mathrm{Ti}^{4+}$ is thus converted to $\mathrm{Ti}^{3+}$ for each lithium ion that is inserted. Upon the charge, Lithium ions and electrons are removed from the lattice, and the $\mathrm{Ti}_{3}^{+}$ideally has full conversion back to $\mathrm{Ti}^{4+}$. This reaction occurs for all of the phases of titania at room temperature, however, anatase is the most active form, 
and is thus the preferred phase for lithium ion storage.

\subsubsection{Rutile}

There are eight known polymorphs of $\mathrm{TiO}_{2}$ : rutile (space group $P 4_{2} / \mathrm{mnm}$ ), anatase $\left(I_{1} /\right.$ amd $)$, brookite $(\mathrm{Pbca}), \mathrm{TiO}_{2}(\mathrm{~B})$ ("Bronze," C2/m), $\mathrm{TiO}_{2}(\mathrm{R})$ ("Ramsdellite," Pbnm), $\mathrm{TiO}_{2}(\mathrm{H})$ ("Hollandite," I4/m), TiO 2 (II) ( Columbite," [ $\left.\alpha-\mathrm{PbO}_{2}\right]$ $P b c n$ ), and $\mathrm{TiO}_{2}$ (III) ("Baddeleyite," $P 2_{1} / c$ ) [62, 63]. Of these, the most common are rutile, anatase, and brookite, with rutile being the most common and stable form. Crystallographically, the ideal rutile structure is a hexagonally close packed oxygen lattice, with octahedrally coordinated metal ions forming edge shared infinite chains along [001] in the tetragonal cell. The chains are crosslinked by sharing corners to form an equal number of identical vacant channels as seen in Figure 2.4. These channels contain two tetrahedral and one octahedral site per metal ion [64]. More specifically, rutile $\mathrm{TiO}_{2}$ is composed of $\mathrm{TiO}_{6}$ octahedra which share edges along the $c$-direction and corners in the $a b$-plane [65].

Because of rutile's crystal structure, it has highly anisotropic elastic and electric properties [65]. Moreover, the diffusion of lithium ions through the rutile lattice is also seen to be highly anisotropic. This is due to the regularity of the shape of the vacancies. The octahedral site is not fully regular, as it has a height of $3.33 \AA$ and a rectangular (rather than square for $100 \%$ regular octahedra) base of $2.96 \times 3.33 \AA$, forcing the tetragonal site to be a distorted pyramid with 2.79 and $2.96 \AA$ sides [66]. Due to the size of the tetragonal vacancies, lithium ions cannot diffuse through without significant lattice distortions. Based on ionic radius, the octahedral sites also must undergo structural deformation in the $a b$-plane, but to a less significant degree [66].

As previously stated, the diffusion of lithium in rutile $\mathrm{TiO}_{2}$ is highly anisotropic, to the extent that diffusion in the $a b$-plane is at least eight orders of magnitude slower than diffusion through the $c$-plane channels $[65,67]$. However, in order 


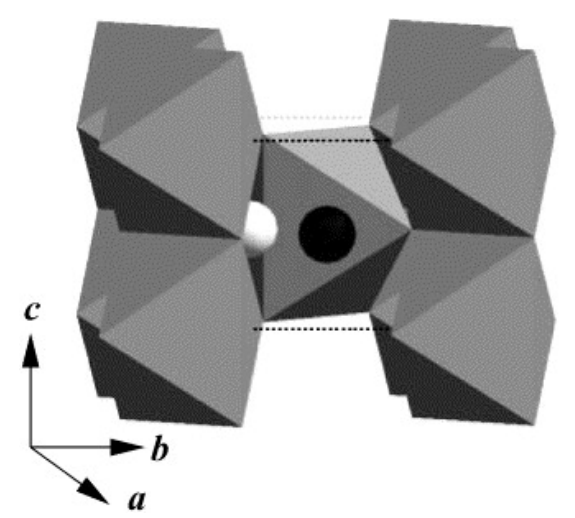

Figure 2.4: Ideal rutile crystal structure showing vacancy channels [65].

to reach the preferred insertion sites, the ions must migrate through the $a b$-plane [68], resulting in nearly one-dimensional diffusion. In bulk (micron-sized or larger) rutile, this means that the fraction of $\mathrm{Li}$ in $\mathrm{Li}_{\mathrm{x}} \mathrm{TiO}_{2}$ is as low as $x=0.03$ [69]. At high temperature $\left(120^{\circ} \mathrm{C}\right)$, bulk rutile intercalates lithium at a $\mathrm{Li} / \mathrm{Ti}$ ratio of $x=0.5$ $[70,71]$. At high operating temperatures, rutile goes through two phase equilibria, showing crystalline structures at $x=0.13$ and $x=0.5$ in $L i_{\mathrm{x}} \mathrm{TiO}_{2}$. Because this operating temperature is not practical, bulk rutile is not viable as an active material for Li-ion batteries.

As previously stated, the anistropy and structure of the rutile crystal causes lithium diffusion to be one dimensional for bulk rutile, limiting its use in LIBs. However, when rutile nanoparticles are employed, lithiation becomes feasible. Particle size reduction results in the decrease of the rate-limiting Li-ion transport distances through the solid-state electrode material. Subsequently, this allows for the general increase of charge and discharge rates. However, in the case of rutile, it means that lithiation can occur at a much larger fraction $[62,69,72]$.

Lithiation of rutile occurs in three stages, as seen in Figure 2.5. The first stage, ranging from $x=0$ to $x=0.15$ is a surface area controlled region [73] that has been suggested to be stored on the surface [62], however has been shown that the 
lithium ions intercalated form a solid solution [72]. Lithiation above $x=0.15$ gives a potential plateau (Region II) that is indicative of two phase behavior [62, 72]. When insertion reaches $x \approx 0.25$, the structure of the rutile crystal begins to convert from its original crystal structure (tetragonal, $\mathrm{P} 4_{2} / \mathrm{mnm}$ ) to a two phase region of both tetragonal and monoclinic crystals $(\mathrm{P} 2 / \mathrm{m})$, and by $x=0.53$, the particles are completely shifted to the monoclinic structure [72]. Region III shows the final structural change that occurs in the crystal lattice. This is the shift to $\mathrm{LiTiO}_{2}$. The structure, which could be either hexagonal or cubic, is still under discussion [73]. The proposed structural change, including hexagonal $\mathrm{LiTiO}_{2}$, can also be seen in Figure 2.5.

Though significant lithiation into nanorutile has only been achieved recently, there have been several undertakings, with various synthesis techniques and morphologies, to lithiate nanorutile at room temperature. Table 2.1 shows select data from various research studies on nanorutile. Hu, et al. [62] achieved a maximum reversible capacity of $150 \mathrm{~mA} \mathrm{hg}^{-1}$, however, this was achieved at very low rate, whereas the capacity at $30 \mathrm{C}$ was only $70 \mathrm{~mA} \mathrm{~h} \mathrm{~g}^{-1}$. Baudrin, et al. [69] also achieved $150 \mathrm{~mA} \mathrm{~h}^{-1}$, but they were testing at an even slower rate $(\mathrm{C} / 60)$. Boghols, et al. [72] was able to obtain the most lithiation $\left(\mathrm{Li}_{0} \cdot 85 \mathrm{TiO}_{2}\right.$, corresponding to $285 \mathrm{~mA} \mathrm{~h} \mathrm{~g}^{-1}$ ), however, their lithiation was perfromed chemically. In fact, the only group that could maintain $100 \mathrm{~mA} \mathrm{~h} \mathrm{~g}^{-1}$ and above at high rate (30C) was Kubiak, et al. [73], who used sol-gel synthesis to produce high surface area nanorutile particles. Their work also showed a maximum charge capacity of approximately $190 \mathrm{~mA} \mathrm{~h}^{-1}$. Zhao, et al [75] used electrospinning to produce woven nanofiber composites, which acted as a self standing, binder-free electrodes with a maximum charge capacity of $200 \mathrm{~mA} \mathrm{~h} \mathrm{~g}^{-1}$. This was achieved at $10 \mathrm{~mA} \mathrm{~g}^{-1}$ (an extremely low rate), but at $1 \mathrm{C}$ rate they were able to achieve a capacity of $\sim 120 \mathrm{~mA} \mathrm{~h} \mathrm{~g}^{-1}$ with a near $100 \%$ Coulombic efficiency for 100 cycles.

${ }^{2}$ Fraction $\mathrm{x}$ in the intercalated compound: $\mathrm{Li}_{\mathrm{x}} \mathrm{TiO}_{2}$ 

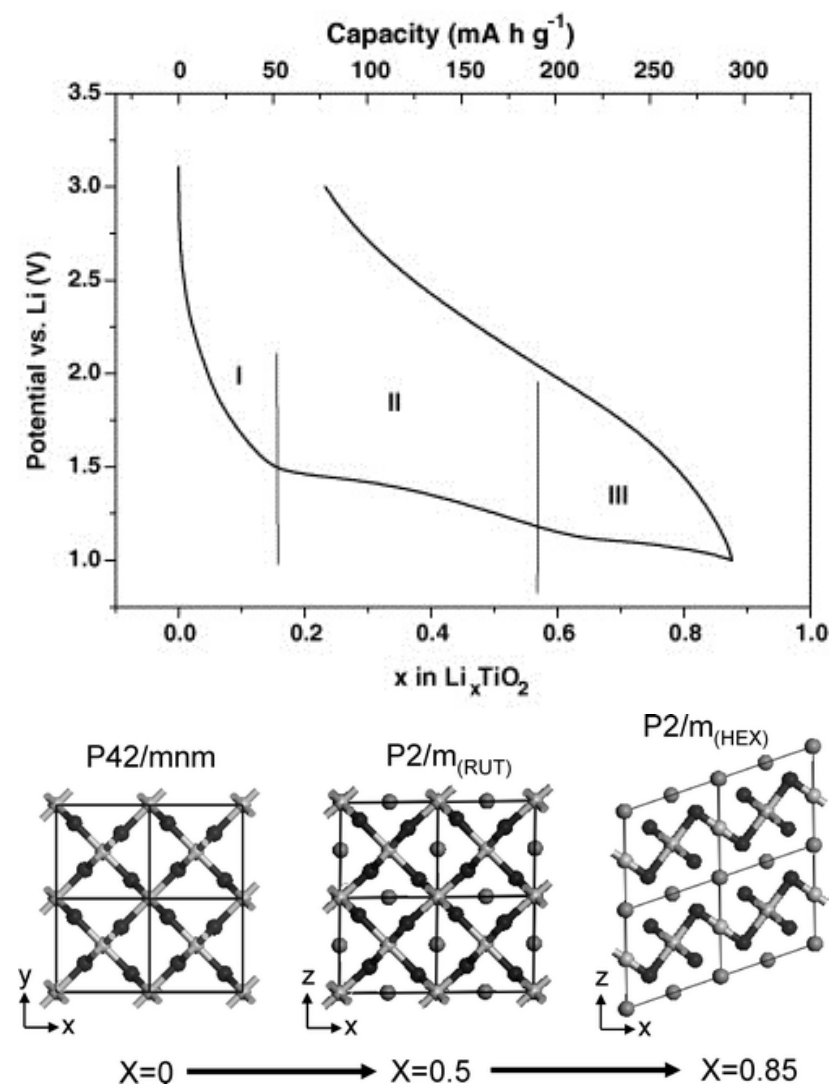

Figure 2.5: Discharge (lithiation) curve for rutile $\mathrm{TiO}_{2}$ showing three distinct regions [73] (top) and the crystallographic changes that accompany each region [72] (bottom). 
Table 2.1: Physical and electrochemical data for rutile nanotitania anode materials.

\begin{tabular}{|c|c|c|c|c|c|}
\hline Group & $\begin{array}{l}\text { Particle Size } \\
\mathrm{nm}\end{array}$ & $\begin{array}{l}\text { Lithium } \\
\text { Insertion }^{2}\end{array}$ & $\begin{array}{l}\text { Maximum Specific } \\
\text { Capacity }\left(\mathrm{mA} \mathrm{h} \mathrm{g}^{-1}\right)\end{array}$ & $\begin{array}{l}\text { Particle } \\
\text { Type }\end{array}$ & $\begin{array}{l}\text { Lithiation } \\
\text { Rate }\end{array}$ \\
\hline Hu, et al. [62] & 10 & 0.5 & 160 & needles & $\mathrm{C} / 20(30 \mathrm{C})$ \\
\hline Chen and Lou [68] & 3 & 0.55 & 187.2 & particles & $1 \mathrm{C}(30 \mathrm{C})$ \\
\hline Baudrin, et al. [69] & 10 & 0.5 & 150 & rods & $\mathrm{C} / 60$ \\
\hline Borghols, et al.[72] & 11 & 0.85 & 285 & needles & $\diamond$ \\
\hline Kubiak, et al. [73] & 5 & 0.6 & 190 & particles & $\mathrm{C} / 5(30 \mathrm{C})$ \\
\hline Reddy, et al. [74] & $5-20$ & 0.6 & 190 & particles & $\mathrm{C} / 10$ \\
\hline Zhao, et al. [75] & $20-200$ & 0.6 & 200 & fibers & $1 \mathrm{C}(10 \mathrm{C})$ \\
\hline
\end{tabular}

\subsubsection{Brookite}

Like rutile, brookite is one of the three most common forms of $\mathrm{TiO}_{2}$. Structurally, brookite is built using the same $\mathrm{TiO}_{6}$ octahedra as rutile, having edge and corner sharing, as well. However, the position of these octahedra forms an orthorhombic structure [76]. The structure of brookite can be seen in Figure 2.6.

The relative stability of the various common phases of $\mathrm{TiO}_{2}$ was examined by Swamy, et al. [77] as well as Zhang and Banfield [78] and Ranade, et al [79]. Mathematical modeling by Swamy, et al. showed that brookite was the second most stable polymorph of $\mathrm{TiO}_{2}$. However, in order to account for the low occurence of natural brookite, other factors must affect the stability more highly. Zhang and Banfield determined that, at the nano scale, crystal size affects stability in the opposite way [78]. Brookite crystals were stable only between 11 and $35 \mathrm{~nm}$; larger and smaller crystals were stable as rutile and anatase, respectively. Ranade, et al. described stability based on surface area and crystal size, thus including crystalline densities as a third factor [79]. The findings indicated that brookite is stable when the surface area is between 592 and $3174 \mathrm{~m}^{2} \mathrm{~mol}^{-1}$---equivalent to 7 to $40 \mathrm{~m}^{2} \mathrm{~g}^{-1}$ or 200 to $40 \mathrm{~nm}$ crystal size.

Because of its metastability, brookite was not believed to be a viable intercalation compound. While it is possible that the channels that form along the $c$-axis in the 


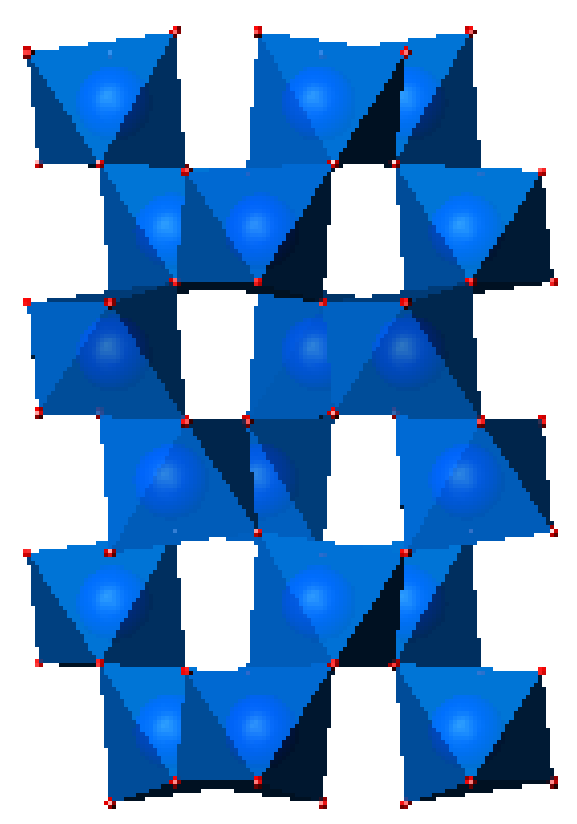

Figure 2.6: Crystal structure of brookite displaying $\mathrm{TiO}_{6}$ octahedra

brookite structure allow lithium diffusion, very low amounts of lithium are inserted in bulk material, as it does in the case of bulk rutile [80]. At the nanoscale, however, brookite does allow lithium diffusion at a larger scale, but the crystal structure behaves differently compared to the other polymorphs of $\mathrm{TiO}_{2}$. Reddy, et al. [81] used ex-situ XRD at various stages of $\mathrm{Li}$ insertion to determine the changes in crystal structure. The results suggested that during intercalation, brookite undergoes an amorphization of the crystal. The crystal loses periodicity as it shifts to allow lithium to sit within the interstitial sites. This is indirectly suggestive of a solid-state solution behavior upon insertion [82]. The XRD pattern of pristine brookite has two peaks next to each other at $2 \theta=25.340^{\circ}$ and $25.689^{\circ}$, respectively. During lithiation the peaks of brookite broaden, and the close proximity of the peaks cause them to appear as if they broaden together, showing one extremely broad peak. This broad peak is what led to the idea of amorphization, but Dambournet, et al. [82] used 
the pair distribution function (PDF) and computational modeling of an electrode lithiated to $x=0.75$ to better determine the physical changes in the lattice. It was determined that upon lithiation, brookite undergoes a $2.9 \%$ and $4.2 \%$ change in the $a$ - and $b$-direction respectively, with a negligible contraction in the $c$-direction $(0.6 \%)$. The PDF study showed that for brookite, Li enters the channels, and forms a distorted octahedron with six $\mathrm{O}$ atoms, as seen in Figure 2.7. This lattice distortion is what causes the peak broadening in the XRD even though the crystal structure itself is not changing. The distortion is reversed upon delithiation, which explains the "partial recovery of crystalinity" described initiatlly [81]. However, to date, no experimental work has been done to verify the claims made by Dambournet.

Part of the reason for the lack of information on brookite and its intercalation properties the physical effects of that lithation on the crystal structure, is that it is difficult to synthesize brookite. Nano-sized brookite has been synthesized for electrochemical cells in a variety of ways, all of which require either extra steps or complex intermediates. Reddy, et al. synthesized a brookite/rutile mixture using thermolysis of $\mathrm{TiCl}_{4}$ at $100{ }^{\circ} \mathrm{C}[81,83]$. Rutile was separated out of the mixture by peptization in $3 \mathrm{M}$ nitric acid followed by centrifugation. The remainder was dispersed in water and centrifuged, then freeze-dried, leaving a $20 \mathrm{~nm}$ particle. This technique was based on that of Pottier, et al. [84] which yielded similar particle size and morphology. In a separate study, Lee, et al. [85] dissolved urea in an aqueous solution containing titanium trichloride $\left(\mathrm{TiCl}_{3}\right)$, and refluxed the solution at $100^{\circ} \mathrm{C}$ for 7 hours. This process produced large aggregates of nanospherical, 10-20 nm crystallites. Dambournet, et al. [87] synthesized a blend of rutile, anatase, and brookite through the thermolysis of $0.9 \mathrm{M}$ titanium oxysulfate $\left(\mathrm{TiOSO}_{4}\right)$ in an aqueous solution. The $\mathrm{Ti}_{2} \mathrm{O}_{3}\left(\mathrm{H}_{2} \mathrm{O}\right)_{2}\left(\mathrm{C}_{2} \mathrm{O}_{4}\right) \cdot \mathrm{H}_{2} \mathrm{O}$ intermediate formed from this process was then thermally decomposed, yielding highly mesoporous micron-size "egg-shaped" particles.

Electrochemically, brookite has shown some promise in the limited number of studies that have been performed. Because of the difficulties in synthesis, and 


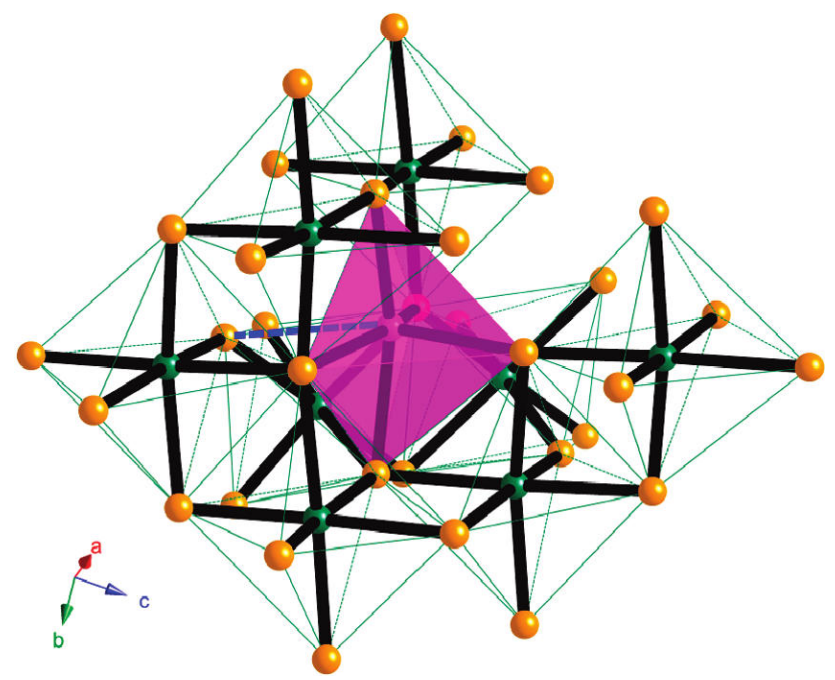

Figure 2.7: Brookite lattice highlighting the lithium environment [82].

its metastable nature, the lithium intercalation characteristics of brookite have only been examined recently. Reddy, et al [81] were the first to examine the electrochemical properties of brookite. They made electrodes by mixing brookite active material with acetylene black and PVDF in a weight ratio of 70:20:10, and were able to achieve a reversible capacity of $170 \mathrm{~mA} \mathrm{~h} \mathrm{~g}^{-1}$ over 40 cycles at a $35 \mathrm{~mA} \mathrm{~g}^{-1}$ (corresponding to a rate of $\mathrm{C} / 10 ; 1 \mathrm{Li}$ in 10 hours). Lee, et al [85] compared pristine brookite (mixed with super $\mathrm{P}$ and PVDF in a ration of 68:20:12) and composites of brookite with multi walled carbon nanotubes (MWCNT) using the same binder ratio, but replacing super $\mathrm{P}$ with composite active material. At $\mathrm{C} / 5$ rate (in this case corresponding to $0.5 \mathrm{Li}$ in 5 hours), reversible capacity of $160 \mathrm{~mA} \mathrm{~h} \mathrm{~g}^{-1}$ was achieved over 50 cycles. A second publication by Lee, et al.[86] focused on the effect on rate capability and capacity retention of brookite/MWCNT composites. This work showed that the electrodes benefited from the addition of MWCNTs with an increase of almost $60 \mathrm{~mA} \mathrm{~h} \mathrm{~g}^{-1}$ at every rate. Immediately after, Reddy, et al. [83] published research focusing on the effect of increased crystallite size on lithiation. They found that as the crystallite (and particle) size increased, 
the capacity decreased significantly. Dambournet, et al. [87], mixed their active material with acetylene black and PVDF in a ratio by weight of 80:10:10, and cycled their electrodes at rates varying from $\mathrm{C} / 25$ to $5 \mathrm{C}$, achieving a reversible capacity of just under $200 \mathrm{~mA} \mathrm{~h} \mathrm{~g}^{-1}$ at $\mathrm{C} / 25$ and approximately $55 \mathrm{~mA} \mathrm{~h} \mathrm{~g}^{-1}$ at $5 \mathrm{C}$. Electrodes were cycled for 20 cycles at $\mathrm{C} / 15$ and achieved stable cycling behavior with the retention of $155 \mathrm{~mA} \mathrm{~h} \mathrm{~g}^{-1}$ [87]. Dambournet, et al. [88] focused later on the effect of annealing temperature on rate capability. They found that while increasing the annealing temperature increased the particle size and incurred losses in capacity at low rate---consistent with previous work done by Reddy, et al. [83]---but at high rate performed better due to higher degree of crystallinity. There are still significant questions that are left unanswered regarding the lithiation of brookite and the effect it has on the crystal structure. While Dambournet, et al. used modeling to explain it, experimental confirmation remains unattained.

\subsubsection{Anatase}

Thermodynamically, rutile is the most stable phase of titania. However, like brookite, anatase is metastable and can both occur naturally and be synthesized. Crystallographically, anatase and brookite differ only in the way the atomic layers are stacked, while anatase and rutile only share a chemical composition. As previously mentioned, all three phases of $\mathrm{TiO}_{2}$ have structures based on the stacking of $\mathrm{TiO}_{6}$ octahedra. Anatase, like rutile, has a tetragonal structure, but its threedimensional structure is built by edge-shared bonding of the octahedra [76]. In this

case, the octahedra share four edges and are arranged in chains that zig-zag, as seen in Figure 2.8a.

Another representation of the crystal structure of anatase, given by three-fold coordination of $\mathrm{O}$ ions in trigonal-planar-type $\mathrm{Ti}_{3} \mathrm{O}$ building blocks is seen in Figure $2.8 \mathrm{~b}$. This representation shows the units conforming to a T-shape. Rutile, on the other hand, displays a Y-shape, while brookite displays both T- and Y-shape. 


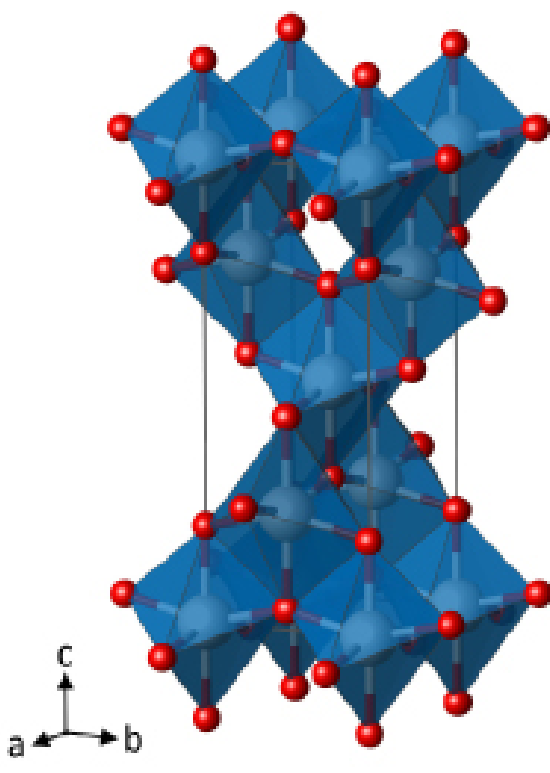

(a)

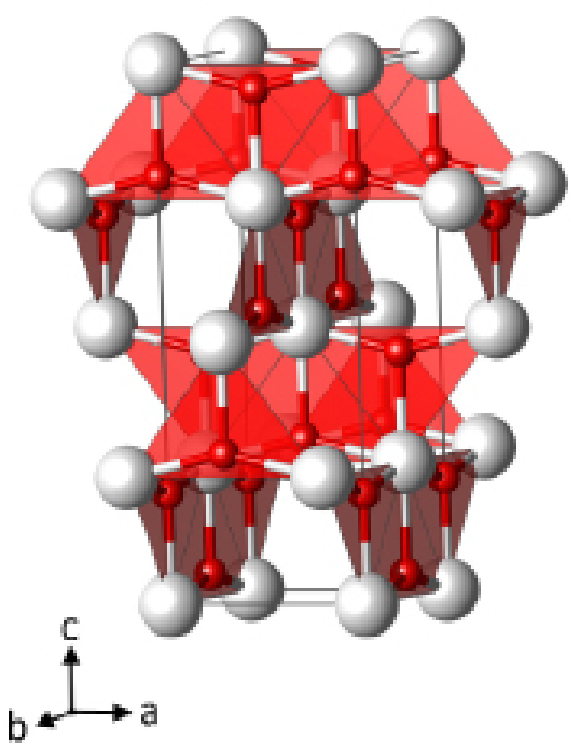

(b)

Figure 2.8: Anatase crystal structure represented by (a) $\mathrm{TiO}_{6}$ polyhedra and (b) planar $\mathrm{Ti}_{3} \mathrm{O}$ building blocks [89]

This model highlights the insertion points for lithium within the crystal lattice. Anatase, unlike rutile and brookite lithiates via a multi step process (Figure 2.9 $[18,40]$ :

1. From $0 \leq x \leq 0.1$ in $\mathrm{Li}_{\mathrm{x}} \mathrm{TiO}_{2}$, a solid solution of lithium and anatase forms $\left(\alpha-\mathrm{LiO} .1 \mathrm{TiO}_{2}\right)$

2. Insertion into the octahedral sites of the bulk anatase crystals, corresponding to the plateau at $1.78 \mathrm{~V}$, indicates a bi-phasic stability between $\alpha-\mathrm{Li} 0.1 \mathrm{TiO}_{2}$ and $\beta-\mathrm{Li}_{\mathrm{x}} \mathrm{TiO}_{2}$

3. As $\alpha$ converts to $\beta$, the voltage drops again and at $1.5 \mathrm{~V} \beta-\mathrm{Li}_{0.5} \mathrm{TiO}_{2}$ is the only stable phase 
4. Below 1.5 V a second bi-phasic diffusion plateau occurs (for $0.6 \leq x \leq 1$ ) where a phase change $\beta-\mathrm{Li}_{0.5} \mathrm{TiO}_{2}$ and $\gamma-\mathrm{Li}_{1} \mathrm{TiO}_{2}$ occurs

5. At $x=1, \gamma-\mathrm{Li}_{1} \mathrm{TiO}_{2}$ is stable and complete lithiation occurs

In some cases, during the formation cycle, the initial solid solution step may go as high as $x=0.22 \mathrm{Li}$ in $\mathrm{Li}_{\mathrm{x}} \mathrm{TiO}_{2}$, however, the loss of capacity in the first cycle suggests that the ions inserted here are mostly irreversible [18]. In later cycles, the storage in the initial voltage drop $(x \leq 0.07)$ arises from an electric double layer, which builds on the surface before being inserted into the octahedral sites. As the lithium inserts into the octahedral sites, a diffusion front occurs as seen in Figure 2.10. At time $t=t_{1}$, the concentration within the lattice reaches $x^{*}$, the critical concentration for a phase change to occur [90]. After this time, lithium entering the lattice inserts within the phase front of $\mathrm{Li}_{\mathrm{x}} \mathrm{TiO}_{2}$. Further, the $\mathrm{Li}_{\mathrm{x}} \mathrm{TiO}_{2}$ undergoes its own phase changes as $x$ increases. At the initial voltage drop, $\mathrm{Li}_{\mathrm{x}} \mathrm{TiO}_{2}$ is considered to be $\alpha-\mathrm{Li}_{\mathrm{x}} \mathrm{TiO}_{2}$, this is the solid solution in the initial step. However, at $1.78 \mathrm{~V}, \alpha-\mathrm{Li}_{\mathrm{x}} \mathrm{TiO}_{2}$ has two-phase coexistence with the orthorhombic $\beta-\mathrm{Li}_{\mathrm{x}} \mathrm{TiO}_{2}$. Individual crystallites can only have a single phase, therefore, the particle structure will shift during the 2-phase plateau region to allow for facile lithiation [40]. This continues until $x=0.5$, when $\beta-\mathrm{Li}_{\mathrm{x}} \mathrm{TiO}_{2}$ is the only stable phase [40]. This phase remains until $x=0.6$ when a second phase shift occurs from $\beta-\mathrm{Li}_{\mathrm{x}} \mathrm{TiO}_{2}$ to $\gamma-\mathrm{Li}_{\mathrm{x}} \mathrm{TiO}_{2}$ with a tetragonal structure, seen as $\mathrm{Li}_{1} \mathrm{TiO}_{2}$ [91]. A two phase stability occurs from $x=0.6$ to $x=1.0$ wherein the $\mathrm{TiO}_{2}$ has fully lithiated and changed to cubic $\mathrm{Li}_{1} \mathrm{TiO}_{2}$. This represents the theoretical maximum capacity that anatase can reach, and has only been reached using chemical lithiation $[40,91]$.

While theoretically it is possible to reach a lithiation point of $x=1$, practically the diffusivion coefficient of $\gamma-\mathrm{Li}_{1} \mathrm{TiO}_{2}$ is orders of magnitude lower than that of $\beta-\mathrm{Li}_{\mathrm{x}} \mathrm{TiO}_{2}$, making it kinetically disadvantaged during electrochemical lithiation [40]. During practical lithiation, at low rate, a hump is seen at $1.5 \mathrm{~V}$ indicating 

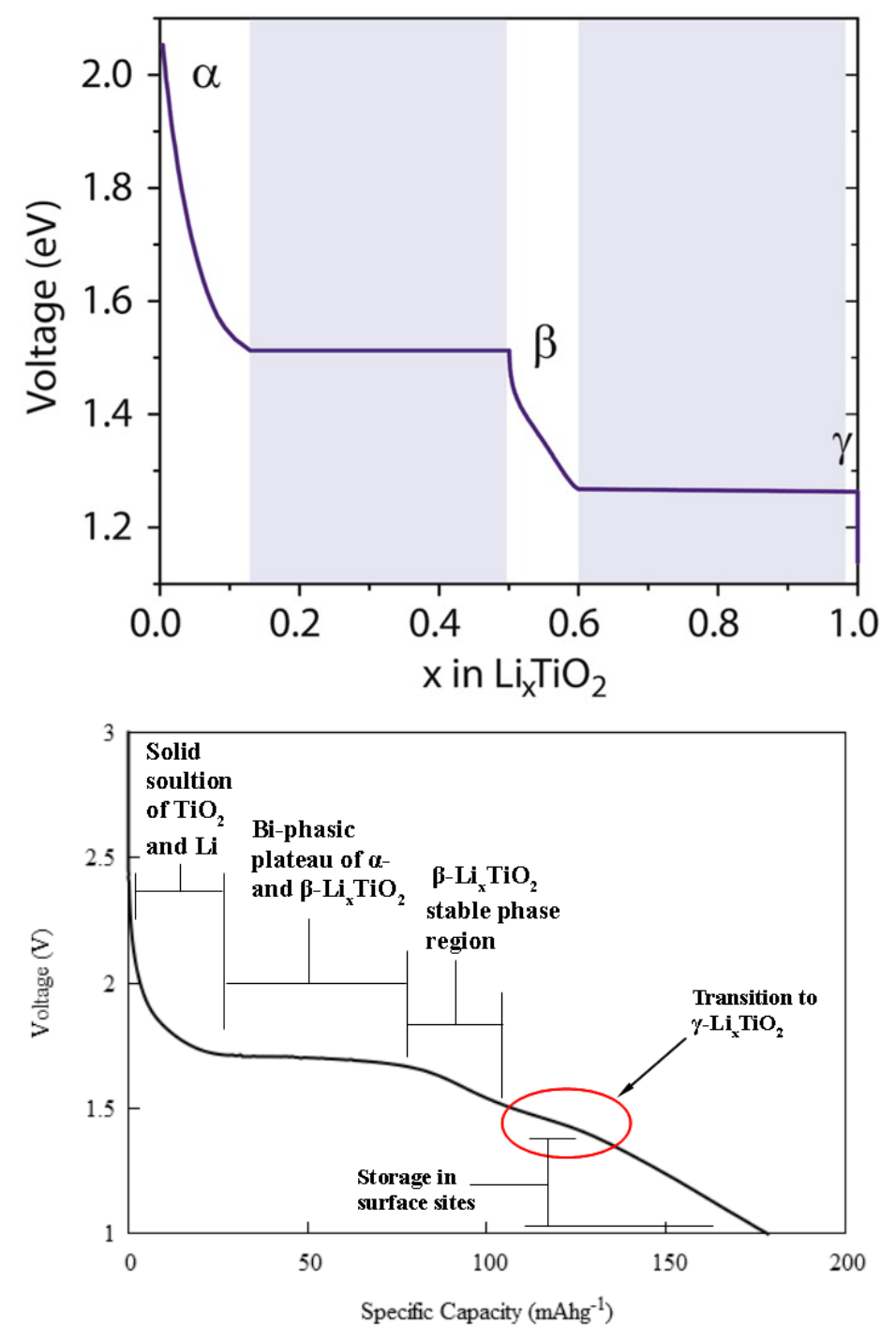

Figure 2.9: Break down of voltage profile depicting theoretical phase transitions of anatase [40] (a) and experimental phase and mechanism changes during lithiation (b). The hump (circled in red) in the experimental profile shows that the phase change from $\beta-\mathrm{Li}_{\mathrm{x}} \mathrm{TiO}_{2}$ to $\gamma-\mathrm{Li}_{1} \mathrm{TiO}_{2}$ does not occur over a long enough period of time to significantly impact the storage capacity. 
that some particles shift from $\beta-\mathrm{Li}_{0.5} \mathrm{TiO}_{2}$ to $\gamma-\mathrm{Li}_{1} \mathrm{TiO}_{2}$ [92], however this accounts for only a minor portion of the capacity, as at this point $\mathrm{Li}^{+}-\mathrm{Li}^{+}$repulsive forces inside the lattice make diffusion-based storage impossible [93]. Instead, the mechanism changes from diffusion to surface storage [18] until the cutoff voltage is reached.
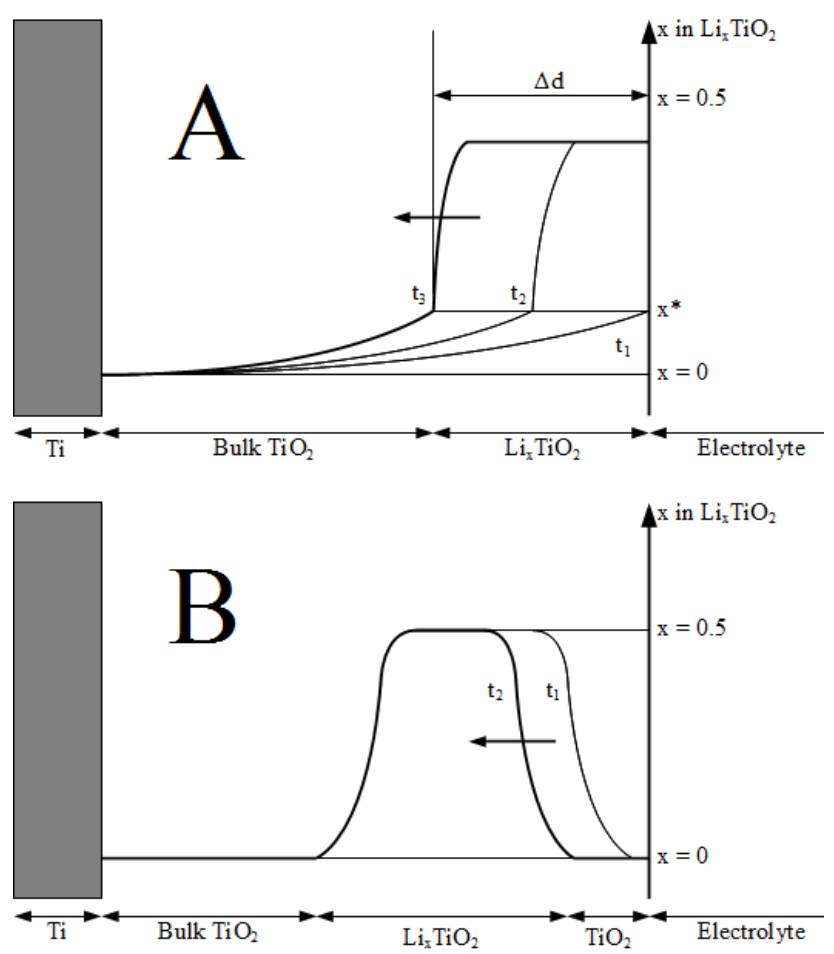

Figure 2.10: Schematic drawing of intercalation process as taken from reference [90]. Panel A shows concentration of $\mathrm{Li}$ in the lattice at various stages of lithation, while Panel B shows the expected Li concentration profile during deintercalation.

It is anatase's ability to easily accommodate a large fraction of lithium that makes it the preferred phase of titania for intercalation electrodes. However, the practical maximum $\left(x=0.5\right.$ in $\left.\mathrm{Li}_{\mathrm{x}} \mathrm{TiO}_{2}\right)$ has forced researchers to look toward nanosizing to take advantage of surface area based charge storage $[95,96]$. While this has 
been achieved by shrinking nanospheres to sizes below $10 \mathrm{~nm}[18,95,97]$, or by making the spheres hollow [98], the largest focus has been on changing the aspect ratio of nanosized particles. A comprehensive list of investigations of physical and electrochemical properties of various morphologies is available in Table 2.2. Chen, et al. [99] investigated hollow microspheres built from $\mathrm{TiO}_{2}$ nanotubes. The spheres were synthesized via hydrothermal processing of amorphous $\mathrm{TiO}_{2}$ precursor. The precursor was prepared by controlled hydrolysis of titanium tetraisopropoxide (TTIP) in an ethanol-water solution. The precursor was mixed with $1 \%$ $\mathrm{H}_{2} \mathrm{O}_{2}$ and $0.2 \mathrm{M} \mathrm{NaOH}$, then hydrolyzed for 12 hours at $180^{\circ} \mathrm{C}$, before being calcined at $450^{\circ} \mathrm{C}$ for 5 hours. The particles achieved $220 \mathrm{~mA} \mathrm{~h} \mathrm{~g}^{-1}$ at $1 \mathrm{C}$, but faded considerably (68\% retention) after 500 cycles. Hemalatha, et al. [100] synthesized $\mathrm{TiO}_{2}$-coated carbon nanotubes (CNTs). To achieve $\mathrm{TiO}_{2}$ coating, functionalized CNTs were suspended in an ethanol solution, and TTIP solution was slowly added. Slow hydrolysis was performed at room temperature by stirring for 12 hours, before the precipitate was obtained, via drying, and calcined at $350{ }^{\circ} \mathrm{C}$ for 48 hours. Four compositions (100-0, 75-25, 50-50, and 25-75 $\left.\mathrm{TiO}_{2}-\mathrm{CNT}\right)$ were synthesized. The nanotubes were cycled at C-rate, with 25-75 achieving the highest capacity of $430 \mathrm{~mA} \mathrm{~h} \mathrm{~g}^{-1}$ after 100 cycles. However, much of that is attributed to the CNTs. As the ratio shifted toward a majority $\mathrm{TiO}_{2}$, the capacity decreased dramatically. Zhu, et al. [101] synthesized a novel rice grain shaped particle via electrospinning a solution of polyvinyl acetate, N,N-dimethyl acetamide, acetic acid, titanium isopropoxide. The product was then sintered at $450{ }^{\circ} \mathrm{C}$ for 1 hour. The nanorods were cycled 800 times at $0.9 \mathrm{C}$ rate $\left(150 \mathrm{~mA} \mathrm{~g}^{-1}\right)$ achieving $81 \%$ capacity retention and an impressive specific capacity of $140 \mathrm{~mA} \mathrm{~h} \mathrm{~g}^{-1}$ at the end of cycling. Yang, et al. [102] synthesized $40 \mathrm{~nm}$ thick nanoflakes by spreading a mixture of stearic acid, low surface tension hydrocarbon and titanium n-butoxide on the surface of high purity water to form a slurry, which was then calcined at $400{ }^{\circ} \mathrm{C}$. The resulting flakes were then cycled at C/20 20 times. Though initially they had a specific capacity of $175 \mathrm{~mA} \mathrm{~h} \mathrm{~g}^{-1}$, it faded to $160 \mathrm{~mA} \mathrm{~h} \mathrm{~g}^{-1}$ before remaining constant until 
the 20th cycle. While these morphologies gave interesting, and desirable results, synthesis scale-up poses problems due to process complexity and use of harmful chemicals.

Overall, however, it is the properties of the nanospheres, whether porous [105, $110,111]$ or dense $[96,97,104,112]$ that provide the best properties. Wang et al. [111] synthesized mesoporous nanospheres by adding an acetic acid solution dropwise into an acetone solution of tetrabutyl titanate to form TiOH. The product was then annealed at $400{ }^{\circ} \mathrm{C}$ to produce porous nanocrystalline titania particles. Their particles were cycled at various rates for 50 cycles (from 20 to $2000 \mathrm{~mA} \mathrm{~g}^{-1}$ ) but 100 cycles at $200 \mathrm{mAg}^{-1}$. The particles achieved a specific capacity of $147 \mathrm{~mA} \mathrm{~h} \mathrm{~g}^{-1}$ (as listed in Table 2.2), but only retained $75 \%$ of its initial capacity. Das, et al. [112] obtained carbon coated nanospheres by heating a clear sol with molar composition of TTIP:CTAB:HCl:sucrose: $\mathrm{H}_{2} \mathrm{O}$ :ethanol = 3.1:1:16:1.84:167:64.2 for 6 hours at $150{ }^{\circ} \mathrm{C}$. The spheres were cycled at $66 \mathrm{~mA} \mathrm{~g}^{-1}$ (0.4C rate) 30 times and achieved a reversible capacity of $270 \mathrm{~mA} \mathrm{~h}^{-1}$. While these types of nanoparticles can be synthesized at the lab scale, commercial scaling is potentially more complicated and costly because of the expensive organic precursors and the acidic environment required [112] or the need for dropwise addition of an acidic solution to produce porous spheres [111]. However, advances in aqueous-based synthesis of $\mathrm{TiO}_{2}$ may provide commercially scalable synthesis routes for not only anatase nanoparticles [12], but other phases and morphologies of $\mathrm{TiO}_{2}[116,117]$

\subsection{Binder-Free Electrode Fabrication}

Commercially, electrodes for lithium ion batteries are fabricated using active material, a conductive additive, and a polymeric binder [118]. For the most part, that binder is poly(vinylidene) fluoride (PVDF). While PVDF is an adequate binder, it must be disolved in a harsh solvent to activate its adhesive properties, namely n-methyl 2-pyrrolidone (NMP). NMP is not only toxic but potentially carcino- 


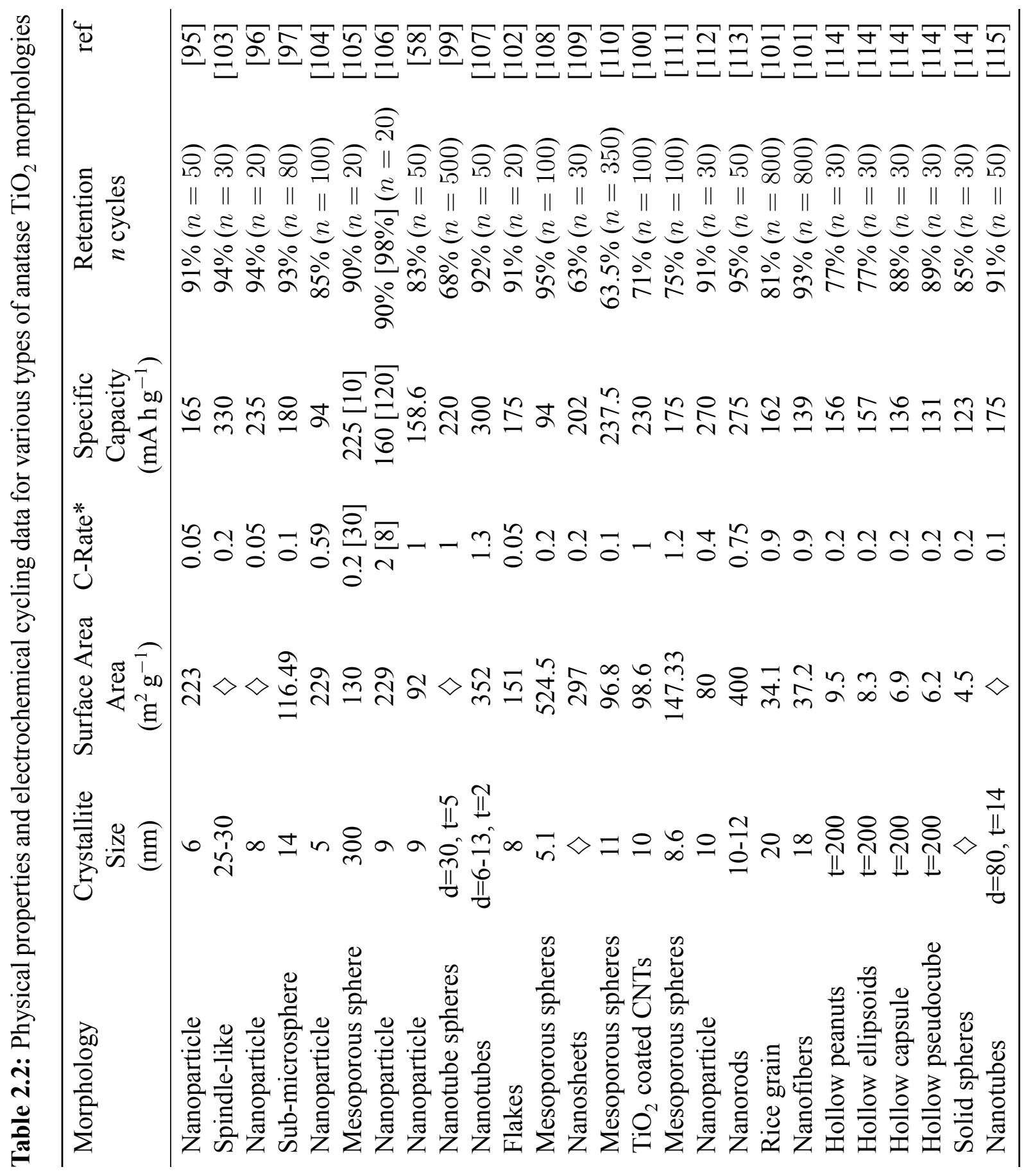


genic, making the binder/solvent combination an undesirable ingredient for battery electrodes. Moretti, et al. [119] has done some work to find different, green binding agents to replace PVDF. The work showed that a combination of sodium carboxymethyl cellulose (CMC) and styrene butadiene rubber (SBR) performed almost as well as PVDF (160.6 vs $163.4 \mathrm{~mA} \mathrm{~h}^{-1}$ ) at $\mathrm{C} / 2$, and better at $5 \mathrm{C}(109 \mathrm{vs}$ $75.2 \mathrm{~mA} \mathrm{~h}^{-1}$. Further, CMC/SBR can be used with deionized water as its solvent, making it safer for use in electrodes. However, both binders are non-electroactive, meaning that they take up mass without contributing to the performance of the cell.

Therefore, focus on removing binders altogether has become the goal. For cathode materials, monolithic $\mathrm{LiCoO}_{2}$ has been studied by Lai, et al. [120], focusing on increasing energy density by using microbatteries. In their work, a monolith was fabricated by milling commercial, battery-grade $\mathrm{LiCoO}_{2}$ and pressing into pellets and sintered for 90 minutes. They provided high energy density $\left(\sim 400 \mathrm{~W} \mathrm{~h} \mathrm{~L}^{-1}\right.$ over 100 cycles and a specific capacity of approximately $150 \mathrm{~mA} \mathrm{~h} \mathrm{~g}^{-1}$ at $\mathrm{C} / 50$ rate. Others have attempted to manufacture monolithic electrodes [121], but could not produce self-standing materials.

The focus on cathode materials is very limited compared to their anodic counterparts. Carbon, the commercial anode material of choice, has been investigated using binder-free techniques to develop flexible anodes [122], high rate electrodes [123], and even supercapacitor electrodes [124]. Other, novel anode materials such as silicon [125], $\mathrm{Fe}_{2} \mathrm{O}_{3}$ [126], even $\mathrm{CuO}$ for Na-ion insertion [127] have been developed. Notably, cobalt oxide $\left(\mathrm{Co}_{3} \mathrm{O}_{4}\right)$ was deposited on copper using electrophoretic deposition [128]. The technique provided a mechanically robust electrode that required neither binder or conductive additives to achieve almost the theoretical capacity of $890 \mathrm{~mA} \mathrm{~h} \mathrm{~g}^{-1}$ after 50 cycles.

$\mathrm{TiO}_{2}$ is also a material of focus for binder-free fabrications. Ortiz, et al. [129] grew $\mathrm{TiO}_{2}$ nanotubes by anodizing Ti-metal potentiostatically $(20 \mathrm{~V})$ in an electrolyte solution of $1 \mathrm{M} \mathrm{H}_{3} \mathrm{PO}_{4}+1 \mathrm{M} \mathrm{NaOH}+0.5 \mathrm{wt} \% \mathrm{HF}$ at room temperature. The nanotubes achieved $210 \mathrm{~mA} \mathrm{~h} \mathrm{~g}^{-1}$ at $\mathrm{C} / 2$ rate and $184.8 \mathrm{~mA} \mathrm{~h} \mathrm{~g}^{-1}$ at $2.5 \mathrm{C}$, retaining 
86 and $75 \%$ of its capacity after 50 cycles. Lee, et al. [130] fabricated electrodes by electrospinning $\mathrm{TiO}_{2}$ nanofibers using a $\mathrm{TiO}_{2}$ precursor solution of poly(vinyl acetate) dissolved in dimethylformamide, titanium(IV) isopropoxide and acetic acid. The nanofibers were then pressed to form a nanoweb. The nanoweb achieved $188 \mathrm{~mA} \mathrm{~h} \mathrm{~g}^{-1}$ after 100 cycles at $0.2 \mathrm{C}$, retaining $90.5 \%$ of its original charge. Tammawat and Meethong [131] also used electrospinning to produce $\mathrm{TiO}_{2}$ nanofibers. In their work, a solution of titanium (diisopropoixe) bis (2, 4-pentanedionate) 75 $\mathrm{wt} \%$ in 2-propanol, polyvinylpyrrolidone, acetic acid, and ethanol was used to form nanofibers that were then calcined at $400-1000{ }^{\circ} \mathrm{C}$. The fibers calcined at $400{ }^{\circ} \mathrm{C}$ showed the best electrochemical properties with a discharge capacity of $160 \mathrm{~mA} \mathrm{~h}^{-1}$ after 50 cycles at $\mathrm{C} / 3$ rate. However, the best results for binder-free $\mathrm{TiO}_{2}$ came from Cheng, et al. [132] who deposited $\mathrm{TiO}_{2}$ particles on a CNT scaffold using a surfactant and water, then mixing them in a $10 \mathrm{M} \mathrm{NaOH}$ solution in an autoclave for 48 hours at $150^{\circ} \mathrm{C}$ to form a CNT- $\mathrm{H}_{2} \mathrm{Ti}_{3} \mathrm{O}_{7}$ intermediate, before thermally decomposing at $500{ }^{\circ} \mathrm{C}$ to obtain a $\mathrm{CNT}-\mathrm{TiO}_{2}$ conformal film. The film achieved an impressive $129 \mathrm{~mA} \mathrm{~h} \mathrm{~g}^{-1}$ after 1000 cycles at $10 \mathrm{C}$ rate, with an equivalent capacity loss of $0.007 \%$ per cycle, indicative of excellent cycling stability at high rate. They are also the only group to have tested binder-free electrodes in full cells (with $\mathrm{LiFePO}_{4}$ as the cathode), and saw almost $100 \%$ capacity retention at $2 \mathrm{C}$ over 400 cycles. However, their fabrication process is deemed very complex for scaled-up application. 


\subsection{References}

[1] D. Linden and T. B. Reddy, Handbook of Batteries, 3rd ed., McGraw-Hill, New York, (2002).

[2] P. J. Hall and E. J. Bain, Energy Policy, 36(12), 4352 (2008).

[3] P. G. Bruce, B. Scrosati, J.-M. Tarascon, Angew. Chem. Int. Edit., 47, 2930 (2008).

[4] M. S. Whittingham, Chem. Rev., 104(10), 4271 (2004).

[5] F. R. Gamble, J. H. Osiecki, M. Cais, R. Pisharody, F. J. Disalvo, T. H. Geballe, Science, 174, 493 (1971).

[6] M. S. Whittingham, J. Electrochem. Soc., 123(3), 315 (1976).

[7] M. Winter and R. J. Brodd, Chem. Rev., 104, 4245 (2004).

[8] V. Etacheri, R. Marom, R. Elazari, G. Salitra, D. Aurbach, Energy Environ. Sci., 4, 3243 (2011).

[9] A. K. Padhi, K. S. Nanjundaswamy, J. B. Goodenough, J. Electrochem. Soc., 144(4), 1188 (1997).

[10] B. Kang and G. Ceder, Nature, 458, 190 (2009).

[11] C. Jiang, E. Hosono, H. Zhou, Nano Today, 1(4), 28 (2006).

[12] C. Charbonneau, R. Gauvin, G. P. Demopoulos, J. Electrochem. Soc., 158(3), H224 (2011).

[13] W. Xu, Z. Wang, Z. Guo, Y. Liu, N. Zhou, B Niu, Z. Shi, H. Zhang, J. Power Sources, 232, 193 (2013).

[14] H. Zhou, D. Li, M. Hibino, I. Honma, Angew. Chem. Int. Ed., 44, 797 (2005).

[15] K. T. Lee and J. Cho, Nano Today, 6, 28 (2011).

[16] S. Nishimura, G. Kobayashi, K. Ohoyama, R. Kanno, M. Yashima, A. Yamada, Nat. Mater., 7, 707 (2008). 
[17] D.Y. Wang, H. Buqa, M. Crouzet, G. Denghengi, T. Drezen, I. Exnar, N. H. Kwon, J. H. Miners, L. Poletto, M. Grätzel, J. Power Sources, 189, 505 (2009).

[18] C. Jiang, M. Wei, Z. Qi, T. Kudo, I. Honma, H. Zhou, J. Power Sources, 166, 239 (2007).

[19] B. E. Conway, Electrochemical Supercapacitors, p. 698, Kluwer Academic/Plenum Publishers, New York, (1999).

[20] D. T. Oakes and E. J. Burcik, Clay Clay Miner.,.4, 225 (1956).

[21] C. Cagel, Charging Kinetics of Electric Double Layer Capacitors, Clemson University (2009).

[22] T. Miettinen, J. Ralston, D. Fornasiero, Miner. Eng., 23, 420 (2010).

[23] R. de Levie, Electrochim. Acta, 8, 751 (1963).

[24] R. de Levie, Electrochim. Acta, 9, 1231 (1964).

[25] B. E. Conway, J. Electrochem. Soc., 138, 1539 (1991).

[26] B. E. Conway, V. Birss, J. Wojtowicz, J. Power Sources, 66, 1 (1997).

[27] V. Augustyn, P. Simon, B. Dunn, Energy Environ. Sci., Accepted (2014).

[28] L. Kavan, J. Solid State Electrochem., Accepted (2014).

[29] D. Galizzioli, F. Tantardini, S. Trasatti, J. Appl. Electrochem., 5, 203 (1975).

[30] I.-H. Kim and K.-B. Kim, Electrochem. Solid St., 4, A62 (2001).

[31] H. Kim and B. N. Popov, J. Power Sources, 104, 52 (2002).

[32] Y. Wang, Z. Wang, Y. Xia, Electrochim. Acta, 50, 5641 (2005).

[33] Y. R. Ahn, M. Y. Song, S. M. Jo, C. R. Park, D. Y. Kim, Nanotechnology, 17, 2865 (2006).

[34] J. W. Long, D. Belanger, T. Brousse, W. Sugimoto, M. B. Sassin, O. Crosnier, Mater. Res. Bull., 36, 513 (2011). 
[35] M. S. Halper and J. C. Ellenbogen, Supercapacitors: A Brief Overview, MITRE Nanosystems Group (2006).

[36] R. Haering and R. MacKinnon, Modern Aspects of Electrochemistry, Plenum Press, New York (1983).

[37] V. Augustyn, J. Come, M. A. Lowe, J. W. Kim, P.-L. Taberna, S. H. Tolbert, H. D. Abruña, P. Simon, B. Dunn, Nat. Mater., 12(6), 518 (2013).

[38] T. Brezesinski, J. Wang, J. Polleux, B. Dunn, S. H. Tolbert, J. Am. Chem. Soc., 131, 1802 (2009).

[39] M. Yao, H. Senoh, T. Sakai, T. Kiyobayashi, J. Power Sources, 202, 364 (2012).

[40] A. A. Belak, Y. Wang, A. Van der Ven, Chem. Mater, 24(15), 2894 (2012).

[41] N. Takami, A. Satoh, M. Hara, T. Ohsaki, J. Electrochem. Soc., 142(2), 371, (1995).

[42] H. Fujimoto, Y. Shoji, Y. Kida, R. Ohshita, T. Nohma, K. Nishio, J. Power Sources, 72, 226 (1998).

[43] E. Peled, J. Electrochem. Soc, 126(12), 2047 (1979).

[44] R. Fong, V. von Schen, J. R. Dahn, J. Electrochem. Soc., 137(7), 2009 (1990).

[45] R. Hanno, Y. Kawamoto, Y. Takeda, S. Ohashi, N. Imanishi, O. Yamamoto, J. Electrochem. Soc., 139(12), 3397 (1992).

[46] A. S. Aricò, P. G. Bruce, B. Scrosati, J.-M. Tarascon, W. van Schalkwijk, Nat. Mater., 4, 366 (2005).

[47] H. Fujimoto, M. Fujimoto, H. Ikeda, R. Ohshita, S. Fujitani, I. Yonezu, J. Power Sources, 93, 224 (2001).

[48] G. Wang, X. Shen, J. Yao, J. Park, Carbon, 47, 2049 (2009).

[49] X. Xiao, P. Liu, J. S. Wang, M. W. Verbrugge, M. P. Balogh, Electrochem. Commun., 13, 209 (2011). 
[50] J. S. Chen, L. A. Archer, X. W. Lou, J. Mater. Chem., 21, 9912 (2011).

[51] A. Magasinski, P. Dixon, B. Hertzberg, A. Kvit, J. Ayala, G. Yushin, Nat. Mater, 9, 353 (2010).

[52] Y. Hwa, C.-M. Park, H.-J. Sohn, J. Power Sources, 222, 129 (2013).

[53] L. Cheng, X.-L. Li, H.-J. Liu, H.-M. Xiong, P.-W. Zhang, Y.-Y. Xia, J. Electrochem. Soc., 154, A692 (2007).

[54] G.-N. Zhu, H.-J. Liu, J.-H. Zhuang, C.-X. Wang, Y.-G. Wang, Y.-Y. Xia, Energ. Environ. Sci., 4, 4016 (2011).

[55] A. Laumann, M. Bremholm, P. Hald, M. Holzapfel, K. T. Fehr, B. B. Iverson, J. Electrochem. Soc., 159(2), A166 (2012).

[56] G. Armstrong, A. R. Armstrong, P. G. Bruce, P. Reale, B. Scrosati, $A d v$. Mater., 18, 2597 (2006).

[57] J. S. Chen and X. W. Lou, Electrochem. Commun., 11, 2332 (2009).

[58] M. Mancini, P. Kubiak, J. Geserick, R. Marassi, N. Husing, M. WohlfahrtMehrens, J. Power Sources, 189, 585 (2009).

[59] S. K. Panda, Y. Yoon, H. S. Jung, W.-S. Yoon, H. Shin, J. Power Sources, 204, 162 (2012).

[60] X. Su, Q.-L. Wu, X. Zhan, J. Wu, S. Wei, Z. Guo, J. Mater. Sci., 47, 2519 (2012).

[61] J. Wang, J. Polleux, J. Lim, B. Dunn, J. Phys. Chem. C, 111, 14925 (2007).

[62] Y. S. Hu, L. Kienle, Y.-G. Guo, J. Maier, Adv. Mater., 18(11), 1421 (2006).

[63] L. Kavan, M. Grätzel, S. E. Gilbert, C. Klemenz, H. J. Scheel, J. Am. Chem. Soc., 118, 6716 (1996).

[64] D. W. Murphy, F. J. Di Salvo, J. N. Carides, J. V. Waszczak, Mater. Res. Bull., 13, 1395 (1978). 
[65] M. Koudriachova, N. Harrison, S. de Leeuw, Solid State Ionics, 157, 35 (2003).

[66] M. Koudriachova, N. Harrison, S. de Leeuw. Phys. Rev. B, 65, 235423-1 (2002).

[67] D. Deng, M. G. Kim, J. Y. Lee, J. Cho, Energ. Environ. Sci., 2, 818 (2009).

[68] J. S. Chen and X. W. Lou, J. Power Sources, 195, 2905 (2010).

[69] E. Baudrin, S. Cassaigon, M. Koelsch, J.-P. Jolivet, L. Dupont, J.-M. Tarascon, Electrochem. Commun., 9, 337 (2007).

[70] B. Zachau-Christiansen, K. West, T. Jacobsen, S. Atlung, Solid State Ionics, 28-30 Part 2, 1176 (1988).

[71] W. J. Macklin and R. J. Neat, Solid State Ionics, 53-56 Part 1, 694 (1992).

[72] W. J. H. Borghols, M. Wagemaker, U. Lafont, E. M. Kelder, F. M. Mulder, Chem. Mater., 20(9), 2949 (2008).

[73] P. Kubiak, M. Pfanzelt, J. Geserick, U. Hormann, N. Husing, U. Kaiser, M. Wohlfarht-Mehrens, J. Power Sources, 194, 1099 (2009).

[74] M. A. Reddy, M. S. Kishore, V. Pralong, V. Caignaert, U. V. Varadaraju, B. Raveau, Electrochem. Commun., 8(8), 1299 (2006).

[75] B. Zhao, R. Cai, S. Jiang, Y. Sha, Z Shao, Electrochim. Acta, 85, 636 (2012).

[76] A. Khataee and G. A. Mansoori, Nanostructured Titanium Dioxide Materials: Properties, Preparation, and Applications, World Scientific, New Jersey (2012).

[77] V. Swamy, J. D. Gale, L. S. Dubrovinsky, J. Phys. Chem. Sol., 62, 887 (2001).

[78] H. Zhang and J. F. Banfield, J. Phys. Chem. B, 104, 3481 (2000).

[79] M. R. Ranade, A. Navrotsky, H. Z. Zhang. J. F. Banfield, S. H. Elder, A. Zaban, P. H. Borse, S. K. Kulkarni, G. S. Whitfield, Proc. Natl. Acad. Sci. USA, 99, 6476 (2002). 
[80] D. W. Murphy, R. J. Cava, S. M. Zahurak, A. Santoro, Solid State Ionics, 9\&10, 413 (1983).

[81] M. A. Reddy, M. S. Kishore, V. Pralong, U. V. Varadaraju, B. Raveau, Electrochem. Solid St., 10(2), A29 (2007).

[82] D. Dambournet, K. W. Chapman, M. V. Koudriachova, P. J. Chupas, I. Belharouak, K. Amine, Inorg. Chem., 50(13), 5855 (2011).

[83] M. A. Reddy, V. Pralong, U. V. Varadaraju, B. Raveau, Electrochem. Solid St., 11(8), A132 (2008).

[84] A. Pottier, C. Chanéac, E. Trone, L. Mazerollesm, and J-P. Joliet, J. Mater. Chem., 11, 116 (2001).

[85] D.-H. Lee, J.-G. Park, K. J. Choi, H.-J. Choi, D.-W. Kim, Eur. J. Inorg. Chem., 2008(6), 878 (2008).

[86] D.-H. Lee, J.-G. Park, D.-W. Kim, Cryst. Growth Des., 8, 4506 (2008).

[87] D. Dambournet, I. Belharouak, K. Amine, Chem. Mater, 22, 1173, (2010).

[88] D. Dambournet, I. Belharouak, J. Ma, K. Amine, J. Mater. Chem., 21, 3085 (2011).

[89] M. Landmann, E. Rauls, W. G. Schmidt, J. Phys.: Condens. Matter, 24(19), 195503 (2012).

[90] R. van de Krol, A. Goossens, J. Schoonman, J. Phys. Chem. B., 103, 7151 (1999).

[91] M. Wagemaker, W. J. H. Borghols, F. M. Mulder, J. Am. Chem. Soc., 129, 4323 (2007).

[92] D. Bresser, E. Paillard, E. Binetti, S. Krueger, M. Striccoli, M. Winter, S. Passerini, J. Power Sources, 206, 301 (2012).

[93] A. G. Dylla, P. Xiao, G. Henkelman, K. J. Stevenson, J. Phys. Chem. Lett., 3, 2015 (2012). 
[94] W. J. H. Borghols D. Lutzenkirchen-Hecht, U. Haake, E. R. H. van Eck, F. M. Mulder, M. Wagemaker Phys. Chem. Chem. Phys., 11, 5742 (2009).

[95] G. Sudant, E. Baudrin, D. Larcher, J.-M. Tarascon, J. Mater. Chem., 15, 1263 (2005).

[96] J. W. Kang, D. H. Kim, V. Mathew, J. S. Lim, J. H. Gim, and J. Kim, J. Electrochem. Soc., 158, A59 (2011).

[97] H.-G. Jung, S. W. Oh, J. Ce, N. Jayaprakash, Y.-K. Sun, Electrochem. Commun., 11, 756 (2009).

[98] F. Zhang, Y. Zhang, S. Song, H. Zhang, J. Power Sources, 196, 8618 (2011).

[99] J. Z. Chen, L. Wang, Y. F. Tang, J. Power Sources, 195, 6893 (2010).

[100] K. Hemalatha, A. S. Prakash, K. Guruprakash, M. Jayakumar, J. Mater. Chem. A, 2, 1757 (2014).

[101] P. Zhu, Y, Wu, M. V. Reddy, A. S. Nair, B. V. R. Chowdari, S. Ramakrishna, $R S C A d v ., 2,531$ (2012).

[102] M.-C. Yang, Y.-Y. Lee, B. Xu, K. Powers, Y. S. Meng, J. Power Sources, 207, 166 (2012).

[103] K.-F. Chiu, K. M. Lin, H. J. Leu, C.-L. Chen, C. C. Lin, J. Electrochem. Soc., 159(3), A264 (2012).

[104] W. Li, F. Wang, S. Feng, J. Wang, Z. Sun, B. Li, Y. Li, J. Yang, A. A. Elzatahry, Y. Xia, D. Zhao, J. Am. Chem. Soc., 135, 18300 (2013).

[105] Y.-G. Guo, Y.-S. Hu, W. Sigle, J. Maier, Adv. Mater., 19, 2087 (2007).

[106] M. Mancini, F. Nobili, R. Tossici, M. Wohlfahrt-Mehrens, R. Marassi, J. Power Sources, 196, 9665 (2011).

[107] S.-J. Park, Y.-J. Kim, H. Lee, J. Power Sources, 196, 5133 (2011).

[108] J. Y. Shin, D. Samuelis, J. Maier, Adv. Funct. Mater., 21, 3464 (2011).

[109] S. B. Yang, X. L. Feng, K. Mullen, Adv. Mater., 23, 3575 (2011). 
[110] W. Wang, Q. Sa, J. Chen, Y. Wang, H. Jung, Y. Yin, Appl. Mater. Interfaces, 5, 6478 (2013).

[111] J. Wang, Y. Zhou, Y. Hu, R. O’Hayre, Z. Shao, J. Mater. Sci., 48, 2733 (2013).

[112] S. K. Das and A. J. Bhattacharyya, J. Electrochem. Soc., 158(6), A705 (2011).

[113] S.-J. Park, H. Kim, Y.-J. Kim, H. Lee, Electrochim. Acta, 56, 5355 (2011).

[114] Y. Wang, X. W. Su, S. Lu, J. Mater. Chem., 22, 1969 (2012).

[115] S. K. Panda, S. Lee, W.-S. Yoon, H. Shin, J. Power Sources, 249, 59 (2014).

[116] C. Charbonneau, R. Gauvin, G. P. Demopoulos, J. Cryst. Growth, 312, 86 (2009)

[117] A. Yasin, F. Guo, G. P. Demopoulos, Materials Research Society Spring Meeting, (2013).

[118] J. Li, C. Daniel, D. Wood, J. Power Sources, 196(5), 2452 (2011).

[119] A. Moretti, G.-T. Kim, D. Bresser, K. Renger, E. Paillard, R. Marassi, M. Winter, S. Passerini, J. Power Sources, 221, 419 (2013).

[120] W. Lai, C. K. Erdonmez, T. F. Marinis, C. K. Bjune, N. J. Dudney, F. Xu, R. Wartena, Y.-M. Chiang, Adv. Energy Mater., 22, E139 (2010).

[121] C. M. Doherty, R. A. Caruso, B. M. Smarsly, P. Adelhelm, C. J. Drummond, Chem. Mater., 21, 5300 (2009).

[122] X. Li, Y. Hu, J. Wang, Y. Li, M. Cai, R. Li, X. Sun, J. Mater. Chem., 22, 18847 (2012).

[123] K. T. Lee, J. C. Lytle, N. S. Ergang, S. M. Oh, A. Stein, Adv. Funct. Mater, 15(4), 547 (2005).

[124] S. Ye, J. Feng, P. Wu, ACS Appl. Mater. Interfaces, 5, 7122 (2013). 
[125] H. Tang, J.-P. Tu, X.-Y. Liu, Y.-J. Zhang, S. Huang, W.-Z. Li, X.-L. Wang, C.-D. Gu, J. Mater. Chem., 2, 5834 (2014).

[126] C. Ban, Z. Wu, D. T. Gillaspie, L. Chen, Y. Yan, J. L. Blackburn, A. C. Dillon, Adv. Mater., 22, E145 (2010).

[127] S. Yuan, X.-L. Huang, D.-L. Ma, H.-G. Wang, F.-Z. Meng, X.-B. Zhang, Adv. Mater., 26, 2273 (2014).

[128] D.-H. Ha, M. A. Islam, R. D. Robinson, Nano Lett., 12, 5122 (2012).

[129] G. F. Ortiz, I. Hanzu, T. Djenizian, P. Lavela, J. L. Tirado, P. Knauth, Chem. Mater, 21, 63 (2009).

[130] S. Lee, J. Ha, J. Choi, T. Song, J. W. Lee, U. Paik, ACS Appl. Mater. Interfaces, 5(22), 11525 (2013).

[131] P. Tammawat and N. Meethong, J. Nanomaterials, 2013, 1 (2013).

[132] J. Cheng, B. Wang, H. L. Xin, C. Kim, F. Nie, X. Li, G. Yang, H. Huang, J. Mater. Chem. A, 2, 2701 (2014). 


\section{Chapter 3}

\section{Binder-Free Fabrication of Nanotitania/Carbon Lithium-ion Intercalation Electrodes}

In the first of the 4 paper-chapters in this thesis, the effect of binder removal is investigated. Polymeric binders are a non-electroactive component of the electrode fabrication process. Removal allows for active material or conductive additive loading in order to achieve higher capacities, and better coulombic efficiency. In removing the binder, a chemo-mechanical paste fabrication, based on the fabrication of dye-sensitized solar cells, was developed in order to adjust for the lack of adhesive binding. This allowed for chemical dispersants to be used which allowed for a more homogeneous paste. By removing the binder, and adding dispersants, conductivity was increased, making lithiation easier. As well, by sintering particles to build a self-supporting structure, better interconnection between particles aided in increasing the capacity by $25 \%$ and the coulombic efficiency to $>90 \%$ as compared to $53 \%$ for standard method electrodes (previously published in: M. J. Sussman, N. Brodusch, R. Gauvin, G. P. Demopoulos, Binder-Free Fabrication of Nanotitania/Carbon Lithium-ion Intercalation Electrodes, Journal of the Electrochemical Society, 165(5), (2013) A3100-3107).

\subsection{Abstract}

A novel fabrication method comprising screen-printing and sintering of nanotitania/carbon paste has been developed allowing the construction of binder-free electrodes with superior lithium-ion intercalation properties. As a model active 
material to demonstrate the advantages of the new fabrication process, commercial P25 nanotitania product was used. The newly fabricated electrodes were compared to those fabricated using the standard binder-based method. Physical characterization demonstrated that the novel binder-free paste provides for significant carbon dispersion due to smaller agglomerate size resulting in enhanced inter-particle (active-conducting) mixing and packing density than the standard one. Cyclic voltammetry and galvanostatic charge/discharge testing proved the novel method-built electrodes to exhibit dramatically improved performance over the standard electrode in terms of conductivity, specific charge capacity, and reversibility. Thus the specific charging (delithiation) capacity of the novel method electrode $\left(92 \% \mathrm{TiO}_{2} / 7.5 \% \mathrm{C}\right)$ was $174 \mathrm{~mA} \mathrm{~h} \mathrm{~g}^{-1}$, compared to $124 \mathrm{~mA} \mathrm{~h} \mathrm{~g}^{-1}$ for the standard method electrode $\left(85 \% \mathrm{TiO}_{2} / 7.5 \% \mathrm{C} / 7.5 \% \mathrm{PVDF}\right)$, representing 103 , and $74 \%$ of the theoretical capacity (corresponding to $\mathrm{Li}_{0.5} \mathrm{TiO}_{2}$ ), respectively. At the same time after 10 cycles, the novel-built electrode exhibited 90-94\% coulombic efficiency and more than $92 \%$ capacity retention while the corresponding values for the standard electrode were only $80-82 \%$ and $53 \%$ respectively.

\subsection{Introduction}

With the growing demand for Electric Vehicles (EV), R\&D in Lithium-Ion Batteries (LIB) has intensified in recent years [1--4]. Current LIBs are limited, among other reasons, by their energy storage, power density, ability to charge and discharge at high rates, but also while LIBs show promise, commercial scale-up and application of these electrochemical energy storage devices has exposed another limiting factor for the acceptance of EVs: cost $[5,6]$. This has driven research to focus on higher performance energy storage solutions [3, 4, 7--10]. Researchers have centered their efforts on experimenting with various electrode chemistries, nanomaterials, and fabrication routes [6, 11--17].

While much research revolves around cathode materials, the anode materials 
have been studied extensively as well. The most common material for anodes is graphite $[18,19]$, however there are problems associated with its safety/long-life performance that is required of LIBs destined for electric vehicles arising from the formation of the well known Surface-Electrolyte Interface (SEI) layer [20]. Graphene [21, 22], $\mathrm{SnO}_{2}$ [12], Si-based [23, 24], $\mathrm{Li}_{4} \mathrm{Ti}_{5} \mathrm{O}_{12}[9,25,26]$, and most recently $\mathrm{TiO}_{2}[12,27--31]$ have attracted attention as alternative to graphite anode materials. Titania, at the nano scale, can potentially find application in both LIBs (as an anode), or in supercapacitors due to its ability to store energy via lithiation as well as via pseudocapacitive behavior at high charge and discharge rates [32, 33], and its lack of an SEI layer. Other attractive features of titania are its non-toxic nature, abundance, and low cost.

The intercalation of $\mathrm{Li}^{+}$ions into the $\mathrm{TiO}_{2}$ lattice is described by the following reaction:

$$
\mathrm{Ti}^{\mathrm{IV}} \mathrm{O}_{2}+\mathrm{x}\left(\mathrm{Li}^{+}+\mathrm{e}^{-}\right) \rightleftharpoons \mathrm{Li}_{\mathrm{x}} \mathrm{Ti}_{1-\mathrm{x}}^{\mathrm{IV}} \mathrm{Ti}_{\mathrm{x}}^{\mathrm{III}} \mathrm{O}_{2}
$$

For anatase, the preferred titania phase [12], lithium intercalation typically reaches $x \approx 0.5 \mathrm{in}_{\mathrm{Li}_{\mathrm{x}}} \mathrm{TiO}_{2}$, which translates to a capacity of $168 \mathrm{~mA} \mathrm{~h} \mathrm{~g}^{-1}$ [34]. Work by Wagemaker, et al. [35] as well as other researchers [36, 37] have provided evidence of formation of lithiated anatase above $x=0.5$, reaching close to $x=1$ or $\mathrm{Li}_{1} \mathrm{TiO}_{2}$, when nanocrystallite size decreases to less than $50 \mathrm{~nm}$. However, these results were achieved using chemical lithiation techniques. Electrochemically attained capacities are much lower than that of the fully lithiated titania, although still possible to be over the theoretical value when slow cycling rates are employed, as seen in the work of Jiang et al. [38]. Belak, et al. [36] reported recently that the fully lithiated titania $\left(\mathrm{LiTiO}_{2}\right)$ is characterized by a diffusion coefficient several orders of magnitude lower $\left(10^{-26} \mathrm{~cm}^{2} \mathrm{~s}^{-1}\right.$ as compared to $10^{-16} \mathrm{~cm}^{2} \mathrm{~s}^{-1}$ for $\mathrm{Li}_{\mathrm{x}} \mathrm{TiO}_{2}$ ) hence explaining why such extreme capacities are practically inaccessible. At the same time, with decreasing nanocrystallite size, in addition to intercalation, 
surface-based charge storage, referred to as pseudocapacitance, may be manifested [29, 32, 33, 39].

Over the years various charge storage properties have been reported for nanosized anatase $\mathrm{TiO}_{2}$ based electrodes. While factors like crystallite size and nanostructure form (particles, nanotubes, nanowires, nanosheets etc.) $[38,40]$ have been found to impact on the storage properties of nanotitania less attention has been placed into the role the method of electrode fabrication itself may have on its performance but equally important on cost of production. Thus, for example Wang et al. [32] prepared their anatase electrode film by drop casting the nanoparticles $(10 \mathrm{~nm}$ size; $150 \mathrm{~m}^{2} \mathrm{~g}^{-1}$ ) onto glassy carbon as substrate (no binder was used), which after air-drying was annealed (calcined) in air at $350-450{ }^{\circ} \mathrm{C}$ and reported a rather modest storage capacity of only $83 \mathrm{~mA} \mathrm{~h} \mathrm{~g}^{-1}$. On the other hand $\mathrm{Hu}$, et al [41] built their electrode by embedding $20 \mathrm{~nm}$ anatase particles into graphene sheets and measured $175 \mathrm{~mA} \mathrm{~h} \mathrm{~g}^{-1}$ charge capacity. However most commonly, electrodes are fabricated with the use of a binder such as poly(vinilydene) fluoride (PVDF). Thus typically the active material, conductive carbon additive, and binder are made into a slurry using a solvent, tape cast onto a current collector substrate and then dried at $\sim 120^{\circ} \mathrm{C}[6]$. For example Chen and Lou [28] using this method built mesoporous electrodes with anatase nanosheets of 20 to $100 \mathrm{~nm}$ dimensions as active material, 20\% Super $\mathrm{P}$ carbon and 10\% PVDF and reported $164.8 \mathrm{~mA} \mathrm{~h} \mathrm{~g}^{-1}$ charge capacity. Similar results $\left(158.6 \mathrm{~mA} \mathrm{~h}^{-1}\right)$ were reported by Mancini et al. [29], who this time used $9 \mathrm{~nm}$ anatase particles with $92 \mathrm{~m}^{2} \mathrm{~g}^{-1}, 12 \%$ super $\mathrm{P}$, and $12 \%$ PVDF but pressed the films before drying. The use of PVDF, however has been questioned [42] because of its high cost and the requirement for a volatile and toxic organic solvent such as n-methylpyrrolidone (NMP). Hence efforts are made to find alternative binders $[42,43]$. Removal of the binder altogether, as investigated in this work, may be an even better strategy, as it will allow for packing more active material into the electrode, lowering the overall cost per kilowatt hour.

It is indeed the scope of the work described herein to develop/describe a novel 
mesoporous electrode fabrication process that not only avoids the use of a binder but also delivers higher capacity and cyclability performance than the standard binder-based method. To demonstrate the advantages of the new fabrication method, commercially available P25 nanotitania is used as model active material. In this method, active material is blended with carbon and formulated as paste by adapting protocols from the fabrication of mesoporous nanotitania electrodes for dyesensitized solar cells $[44,45]$. Electrodes prepared by screen printing and sintering, employing the novel method are compared, both in terms of physical characterization and electrochemical performance, to electrodes prepared using the standard binder-based electrode method.

\subsection{Experimental}

For this work, commercially available P25 titania (Evonik Aeroxide ${ }^{\circledR} \mathrm{TiO}_{2}$ ), was used as the active material because its properties are well studied [46, 47]. Acetylene black was used as the conductive component. For electrodes fabricated with the standard method, poly(vinylidene) fluoride (PVDF 6020) (Solvay) was used as a binder, and 1-methyl 2-pyrrolidone (NMP) (Sigma Aldrich) as the solvent. Fluorine-doped Tin Oxide (FTO) glass (Sigma Aldrich) was used as a current collector for all electrodes. The FTO glass used was $2 \mathrm{~mm}$ thick with a sheet resistance of $\sim 7 \Omega \mathrm{cm}^{-2}$.

\subsubsection{Electrode Fabrication}

Electrodes were fabricated using two different methods, standard and novel. Applying the standard method, electrodes were made by mechanically mixing P25 powder, acetylene black, and PDVF in NMP to produce a paste with constitution $85 \mathrm{wt} \% / 7.5 \mathrm{wt} \% / 7.5 \mathrm{wt} \%$. The paste was deposited on FTO glass substrate and dried for 24 hours at $120^{\circ} \mathrm{C}$. 
Figure 3.1 provides a flow chart depicting the basic steps followed to fabricate electrodes using the novel method. P25 was dry mixed with acetylene black, and then ethanol was added as a diluent and dispersant for the carbon. The slurry was alternately mixed mechanically and by ultrasonication, after adding $\alpha$-terpineol as a rheological agent, and ethyl cellulose as a spacer. ${ }^{1}$ The slurry was condensed to the desired viscosity, screen printed on FTO glass, dried and sintered at $450{ }^{\circ} \mathrm{C}$ by employing a specific heating profile as reported elsewhere [48, 49]. Sintering under these conditions allowed not only for inter-particle necking, but also for the destruction and removal of the non-active paste ingredients: $\alpha$-terpineol and ethyl cellulose. Before the slurry was spread on the FTO glass, the latter was cleaned using UV/Ozone, and then pre-treated by soaking in $50 \mathrm{mM} \mathrm{TiCl}_{4}$ for 30 minutes at $70^{\circ} \mathrm{C}$ to improve film adhesion. After sintering, the electrode film was post-treated by soaking in $50 \mathrm{mM} \mathrm{TiCl}_{4}$ for 30 minutes at $70^{\circ} \mathrm{C}$ and then sintered again at $450{ }^{\circ} \mathrm{C}$ in order to ensure optimum inter-particle necking. Two electrode compositions were prepared using the novel method: the first having the same active material content as the standard electrode ( $85 \mathrm{wt} \%$ P25 and $15 \mathrm{wt} \%$ carbon) and the other having the same carbon content as the standard one, i.e. $92.5 \mathrm{wt} \%$ P25 and $7.5 \mathrm{wt} \%$. Both the standard and novel method fabricated electrodes were degassed in a vacuum oven at $120^{\circ} \mathrm{C}$ for $24 \mathrm{~h}$ before being put in the glovebox.

\subsubsection{Physical Characterization}

Physical characterization was performed both on the P25 powder, and on the electrode films. X-ray Diffraction (XRD), performed with a Bruker D8 Discover Diffractometer equipped with a copper target $\left(\lambda_{C u K 1}=0.15406 \mathrm{~nm}\right)$, was used to determine the type of crystal phases present in the P25 powder, and Rietveld refine-

\footnotetext{
${ }^{1}$ The function of the rheologic agent $\left(\alpha\right.$-terpineol, $\left.\mathrm{C}_{10} \mathrm{H}_{18} \mathrm{O}\right)$ is to maintain viscosity and bind the colloidal suspension during the formulation process, while the ethyl cellulose acts to form the pore structure and disperse $\mathrm{TiO}_{2}$ in suspension. Both are removed in the sintering process leaving the as-sintered electrode binder-free. Chemical structures associated with $\alpha$-terpineol and ethyl cellulose are found in Figure B.1
} 


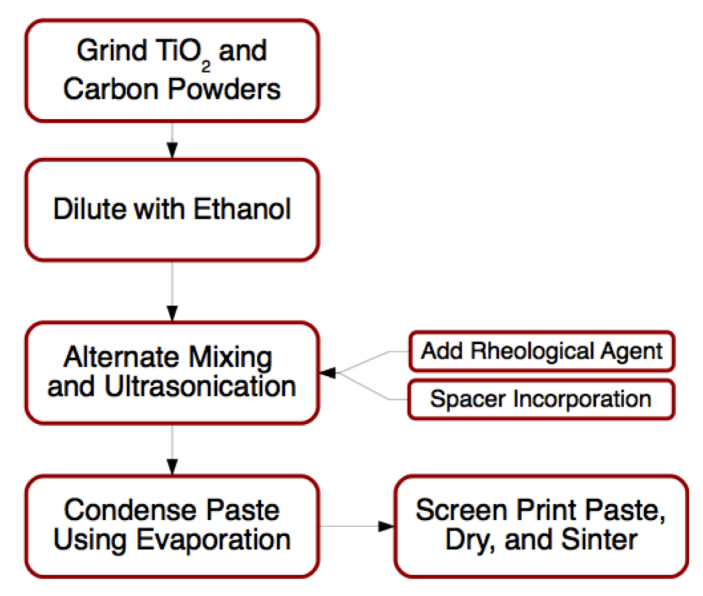

Figure 3.1: Flowchart of novel electrode fabrication method.

ment was performed to determine the quantitative phase composition. BrunauerEmmett-Teller (BET) surface area was used to verify particle size, surface area, and film pore size distribution, using a Micrometrics TriStar 3000 apparatus under nitrogen-controlled atmosphere. All samples weighed approximately $0.1 \mathrm{~g}$ and were degassed for 40 hours at $300^{\circ} \mathrm{C}$ prior to nitrogen physisorption.

Morphological characterization was done with a Hitachi SU-8000 cold fieldemission scanning electron microscope (CFE-SEM) (Hitachi, Rexdale, Canada) equipped with an Xmax silicon drift detector (SDD) as the energy dispersive spectrometer (EDS) and an electron backscatter diffraction (EBSD) Nordlys II system, both provided by Oxford Instruments (Oxford Instruments, Concord, US). The EBSD system was used to acquire the Kikuchi pattern. The diffuse background was acquired with a large number of particles and subtracted to the raw pattern by flat fielding.

Secondary electron (SE) imaging was carried out at an accelerating voltage of 0.5 $\mathrm{kV}$ using the deceleration mode of the microscope. Scanning transmission electron microscopy (STEM) imaging was carried out at an accelerating voltage of $30 \mathrm{kV}$. The transmitted electrons (TE) were collected by means of an Yttrium Aluminum 
Garnet (YAG) type scintillator placed at the bottom of the specimen chamber, below the thin specimen. A set of apertures was used to select the collection semi-angle for bright-field (BF) imaging, which was $10 \mathrm{mrad}$.

Transmission electron forwardscatter diffraction (t-EFSD) in the SEM [50--52] was used to characterize the crystallography of the $\mathrm{TiO}_{2}$ material. In this technique, a thin specimen is placed above the commercial EBSD camera and a Kikuchi pattern is recorded. The pattern is composed of pairs of Kikuchi lines which are representative of the angular distribution of the specific Bragg diffracted transmitted electron [53]. Phase identification is then automatically made with the conventional EBSD software by comparing simulated patterns of expected phases with the experimental pattern [54]. Simultaneous t-EFSD, STEM-BF imaging and X-ray microanalysis by EDS was performed for this work, and additional information can be found in Brodusch et al. [52].

The thin specimen used for STEM imaging and t-EFSD was prepared as follows: the coating was scrapped off from the glass substrate, embedded in LR White resin (Electron Microscopy Sciences, Hatfield, US) and cured in a vacuum oven at $60^{\circ} \mathrm{C}$ for $48 \mathrm{~h}$. After trimming, the polymerized block was cut with a diamond knife with a LKB Ultrotome NOVA (LKB, Bromma, Sweden) ultramicrotome. The sections were retrieved on a TEM copper grid with a lacey carbon film. The sections thicknesses were determined from the resin color under reflected light (silver to gold) and were estimated to be between 70 and $90 \mathrm{~nm}$.

\subsubsection{Electrochemical Characterization}

Electrochemical testing was performed with a Biologic VSP Potentiostat/Galvanostat/EIS system using three-electrode cells constructed in an argon-filled glovebox. Titania anodes were the working electrodes, while lithium foil was used as both the reference and counter electrodes. The working electrode material was applied to the substrate and then shaped into approximately $2 \times 1.5 \mathrm{~cm}$ areas. The 
electrolyte used in this work was a solution of $1 \mathrm{M} \mathrm{LiPF}_{6}$ in EC:DMC:DEC (2:2:1 by weight) mixed solvent (BASF).

Cyclic voltammetry (CV) was performed on both the standard and novel fabrication method electrodes. $\mathrm{CV}$ was performed from 1 and $3 \mathrm{~V}$ at a scan rate of $0.1 \mathrm{mV} \mathrm{s}^{-1}$. CV was performed for 5 cycles, in order to attain a representative voltammogram.

Galvanostatic charge/discharge testing was also performed on electrodes for both types of fabricated electrode. A current of $168 \mathrm{~mA} \mathrm{~g}^{-1}$ (representative of $1 \mathrm{C}$ rate) was used for both charging and discharging. Cells were first discharged to insert Li-ions into pristine $\mathrm{TiO}_{2}$, and then charged. This testing was carried out over 11 cycles in order to observe 10 cycles after the initial discharge and charge couple.

\subsection{Results and Discussion}

\subsubsection{Powder Characterization}

Though P25 is commonly used, its composition is not reported by Degussa (now Evonik). What is known is that $\mathrm{P} 25$ consists of more than $70 \%$ anatase with some rutile, although the compositions may vary somewhat from batch to batch [47]. The energy storage properties of anatase and rutile are quite different, thus performance of the material is dependent on the relative ratios of the phases present in the batch used $[55,56]$. In order to fully analyze the electrochemical data and characterize the structure of the electrodes, physical analysis of the P25 batch used in this work was performed.

XRD analysis involving Rietveld refinement, as described in detail elsewhere [57], determined the following P25 phase composition: $86 \%$ anatase, $11 \%$ rutile and $3 \%$ brookite. In addition to $\mathrm{XRD}$, identification of the different phase nanoparticles was made by t-EFSD. Figure 3.2 shows a STEM-BF image (top left) and a SE image (top right) recorded at $30 \mathrm{kV}$. T-EFSD patterns were acquired on the particles pointed 
by the arrows. Because the crystal structures for each phase of titania give a unique t-EFSD pattern, the twinned particle in the STEM image was clearly identified as anatase whereas the particle on the SE image was found to be rutile. Nanoparticle differences among the major anatase and minor rutile phase present can be seen in Figure 3.3. Due to the small amount present, brookite was difficult to discern. Anatase nanoparticles can be seen to be smaller and rounder (around 20-25 nm), while rutile particles are somewhat larger and more angular (typically 50-60 nm). These numbers are in general agreement with the BET surface area measurements conducted that yielded $50 \mathrm{~m}^{2} \mathrm{~g}^{-1}$, corresponding to an average particle size of 30 $\mathrm{nm}$.

Carbon black, unlike P25 is amorphous. Therefore, because it does not exhibit any Kikuchi bands it is easy to identify these particles through phase mapping (the amorphous phase would appear completely black in the map). However, this kind of mapping on nanoparticles requires long acquisition time and several maps are necessary to evaluate reliably the phase distribution. Because the STEM-BF signal is a mix of mass-thickness and diffraction contrasts, amorphous carbon easily stands out in STEM mode from the crystalline P25 [57]. The particles have a distinctly different morphology from that of P25. This makes them easier to identify in both STEM-BF and SE modes. Finally, the acetylene black variety used in this work was determined to have a surface area of approximately $60 \mathrm{~m}^{2} \mathrm{~g}^{-1}$.

\subsubsection{Electrode Film Structure}

The different characteristics of the two electrode fabrication methods translate into significant electrode structure differences that were found to influence performance. The standard fabrication method is a rather mechanical process, in which the powdered active material, conductive additive and binder are mixed with solvent to form a thick slurry that is deposited on a substrate followed by drying. This process works well to build a mesoporous electrode structure thanks to the role of 

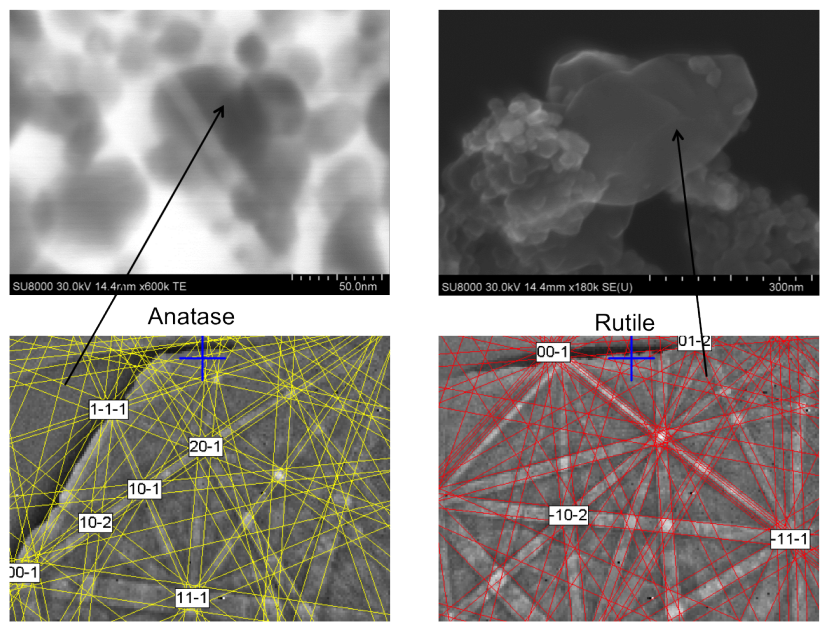

Figure 3.2: STEM-BF and SE images with t-EFSD patterns for anatase (left) and rutile (right) phase particles.

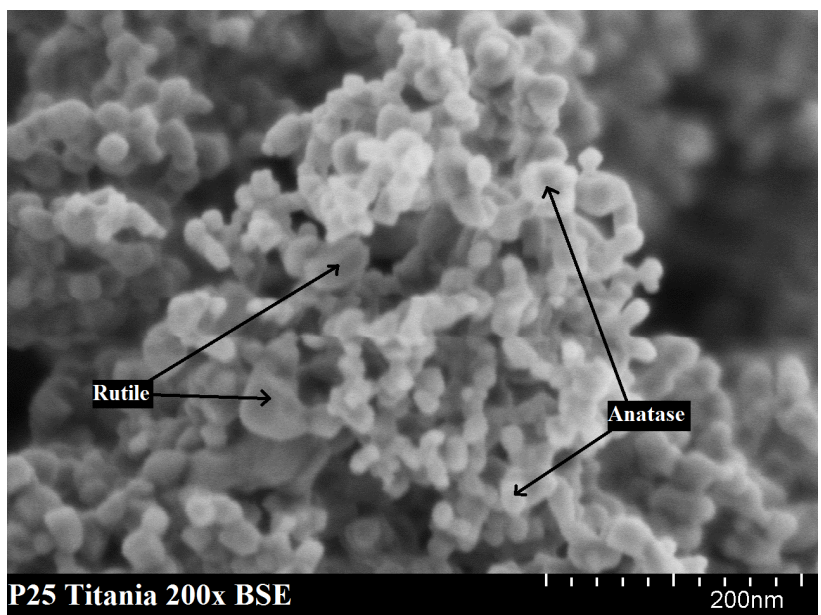

Figure 3.3: Electron micrograph of $\mathrm{P} 25$ powder at 200,000x magnification with rutile and anatase particle varieties labelled. 
the polymeric binder. However, the binder is non-electroactive, translating to lower energy density. Moreover, mechanical mixing and the low drying temperature $\left(120^{\circ} \mathrm{C}\right)$ used may not provide uniform dispersion and inter-particle contacting necessary for good electronic conductivity.

The novel fabrication method aims to eliminate these deficiencies by removing the polymeric binder, and replacing it with a different chemical paste formulation suitable for high temperature sintering $\left(450^{\circ} \mathrm{C}\right)$. By sintering the film, inter-particle necking takes place creating a structural scaffold. This scaffolding gives mechanical stability to the particles and eliminates the need for a polymeric binder. Figure 3.4 shows top-view images of the two types of electrodes. In Figure 3.4(a), the standard electrode shows a somewhat rough surface. This is better seen with the higher magnification micrograph in Figure 4(b). There is clear evidence of large agglomerates resulting in a heterogeneous structure. By contrast, the novel method electrode (Figures 3.4(c) and 3.4(d) have exhibited a more homogeneous surface structure. The 500x magnification image of the novel method electrodes (Figure 3.4(c)), shows small cracks on the otherwise homogeneous surface. It is likely that these cracks formed during sintering as a result of the escape of volatile organics from the destruction of paste ingredients like $\alpha$-terpineol and ethyl cellulose. It is postulated that the new paste formulation, with its well-engineered colloidal constitution (in terms of rheological agent and spacer), accompanied by the controlled sintering protocol is responsible for rendering the novel film with significantly improved homogeneous structure.

In order to further probe the film structure high resolution images were obtained in STEM-BF mode in the CFE-SEM. Contrary to SE bulk imaging, STEM was applied on thin slices of 70 to $90 \mathrm{~nm}$ (approximately 2-3 particles thick) cut with an ultramicrotome. Figure 3.5 shows slices of standard and novel method electrodes in STEM mode. Comparison of the three slices clearly indicates significant differences in terms of particle dispersion and packing among the three electrodes. Note that the large amount of open space that characterizes the standard electrode is an indicator 


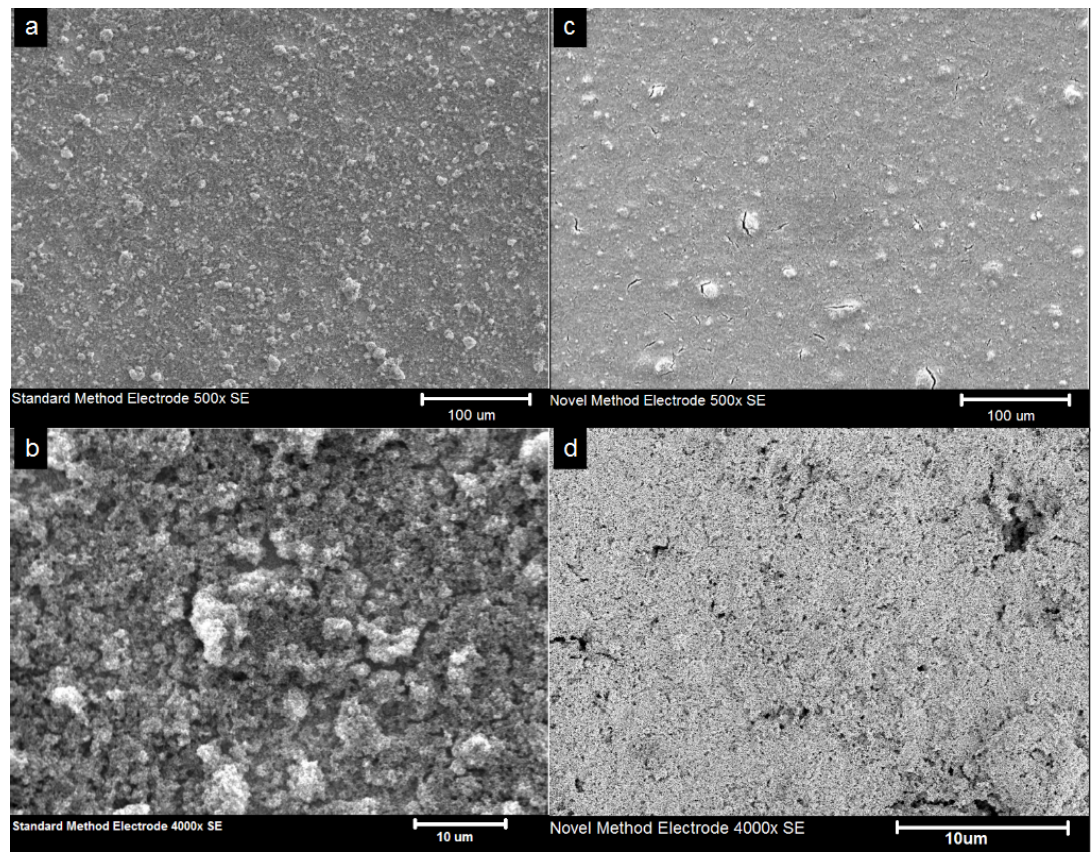

Figure 3.4: Scanning electron micrographs of the two electrodes at different magnifications: Standard method electrode at 500x (a) and 4000x (b); novel method electrode $(7.5 \mathrm{wt} \% \mathrm{C})$ at 500x (c) and 4000x (d). 
of increased film roughness. Looking at the slices for both electrodes fabricated using the novel method (Figures 3.5(b) and 3.5(c)), there is an obvious increase in packing, as more of the slice area is occupied by $\mathrm{TiO}_{2}$ nanoparticle interconnections, as compared to the standard method. Despite, however, the apparent higher packing density observed in the case of the novel electrode, its surface area and pore size distribution (as determined from the BET measurements) was similar to that of the binder-based electrode, namely 50 versus $48 \mathrm{~m}^{2} \mathrm{~g}^{-1}$ and $14-18 \mathrm{vs}$. $16-19 \mathrm{~nm}$, respectively. In other words because of the spacer used in the formulation, the mesoporous structure was maintained.

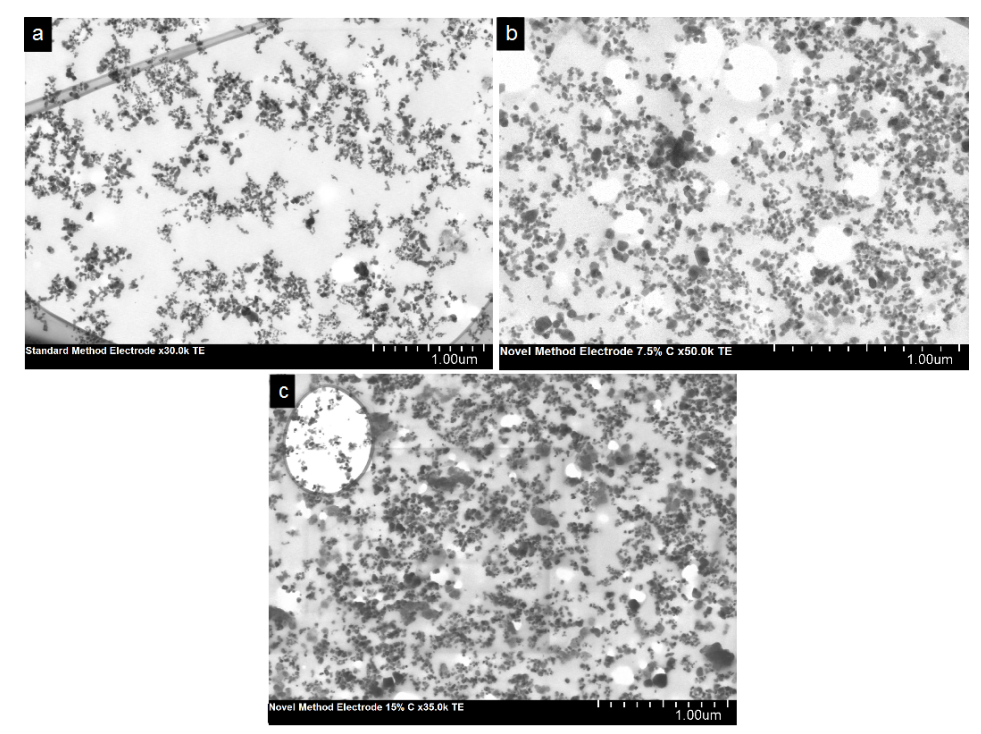

Figure 3.5: STEM-BF images of 70 to $90 \mathrm{~nm}$ thick electrode slices of the standard (a), $7.5 \mathrm{wt} \%$ carbon novel (b), and $15 \mathrm{wt} \%$ carbon novel method electrodes (c).

As mentioned earlier, in connection to Figure 3.4, the binder-based electrode was observed to have significant carbon agglomeration occurrence. In order to substantiate this observation, STEM mode images (at higher magnification) were used to quantify the agglomerate size (described in absolute area) and the number 
of agglomerates per unit area. Figure 3.6 shows a histogram of the agglomerate size distribution for carbon in both electrodes. For the novel method electrode, most of the agglomerates were $0.01 \mu \mathrm{m}^{2}$ or smaller, while for the standard method electrodes there was a wider (and larger) size distribution. As a result, there were fewer (but larger) carbon agglomerates per unit area in the case of the standard electrode in comparison to the novel electrode; for example, there were approximately 2.5 carbon agglomerates per $\mu \mathrm{m}^{2}$ in the standard method electrodes versus 4.8 agglomerates per $\mu \mathrm{m}^{2}$ for the $7.5 \mathrm{wt} \% \mathrm{C}$ novel electrode. This means that there is better carbon dispersion in the novel electrode providing for more contact points between the conductive additive and the active materials, i.e. creating a better percolation network.

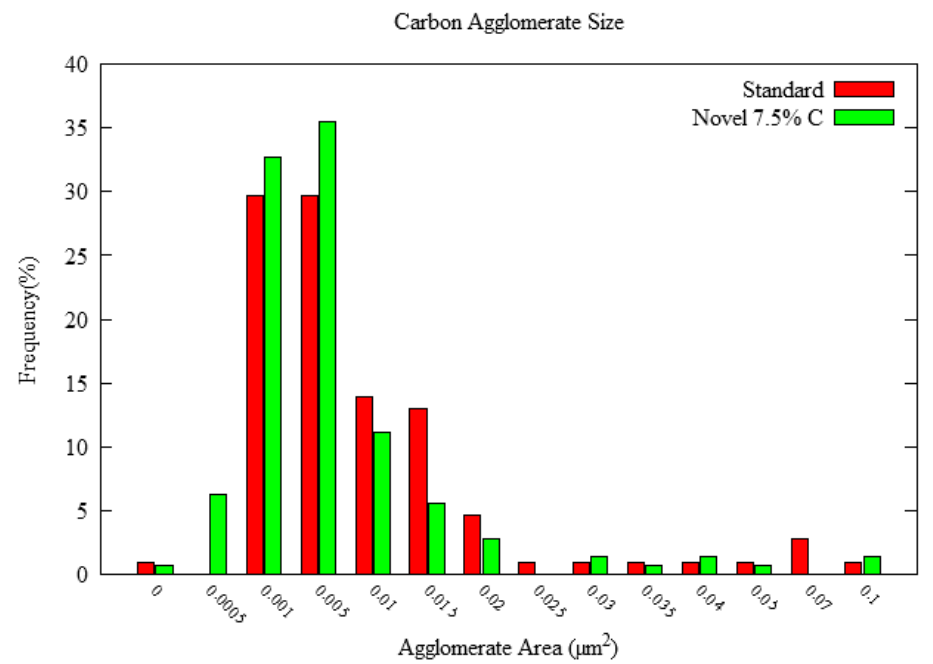

Figure 3.6: Comparison of carbon agglomerate size distribution normalized to percentage of total carbon agglomerates for the two electrodes. ${ }^{2}$

When the films are observed in the CFE-SEM in SE imaging mode at high

\footnotetext{
${ }^{2}$ Measurement of agglomeration distribution was performed on 30 slices from various areas of the electrode film.
} 
magnifications, the nonstructural differences become more apparent (Figure 3.7). In comparison to Figure 3.7(a) depicting the standard method electrode nanostructure, the novel electrode film images (Figure 3.7(b) and 3.7(c)) clearly show, as a result of the applied sintering protocol, a monolithic structure to have forms resulting in an interconnected percolation network of titania. The particles are connected to each other via necking rather than single point contact exhibited by the standard method electrode. As a result, not only a continuous high conductivity network forms but also a mechanically robust structure that can better accommodate strain during lithium-ion insertion and extraction processes, as shown in the next section discussing electrochemical performance.

\subsubsection{Electrochemical Performance}

Figure 3.8(a) shows the reproducible (second to fifth scans) cyclic voltammograms for the standard (binder-based) and novel (binder-free) electrodes. Both are seen to display intercalation peaks at approximately 1.7 (lithiation) and $2.1 \mathrm{~V}$ (delithiation), which is in general agreement with the values found in the literature [58]. Delithiation should occur at approximately $2 \mathrm{~V}$, but it is likely that the shift in delithiation peak seen in all three samples was due to the FTO substrate. The glass had a sheet resistance of $\sim 7 \Omega \mathrm{cm}^{-2}$, which is high compared to commonly used metal substrate. All electrodes exhibited slight differences between the first $\mathrm{CV}$ cycle and the subsequent ones that were all the same. This can be evaluated by referring to Figure 3.8(b), where the first and fifth cycles are shown for the novel method fabricated electrode with $7.5 \mathrm{wt} \%$ carbon. A shallow plateau appears in the first cycle after the lithiation peak $(\sim 1.4 \mathrm{~V})$. This shallow plateau disappears in the second cycle, which had identical shape to the fifth cycle shown in the Figure. The appearance of the shallow plateau in the first cycle may be attributed to gas evolution stemming from trace humidity remaining after degassing in the vacuum oven. 


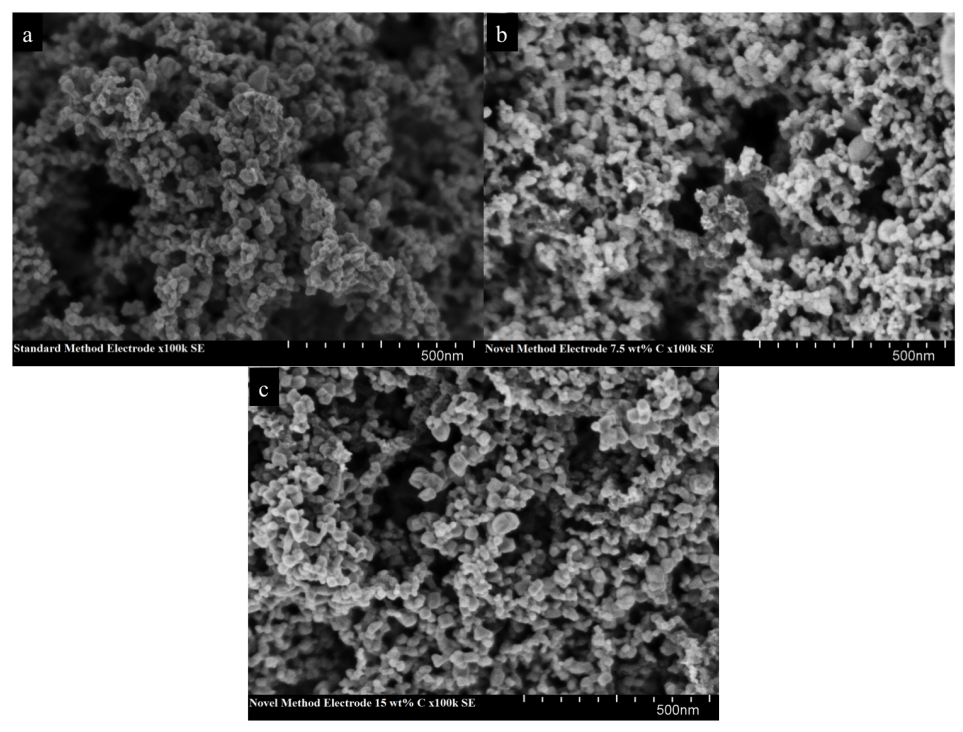

Figure 3.7: High magnification scanning electron micrographs of the standard (a), $7.5 \mathrm{wt} \%$ carbon novel (b), and $15 \mathrm{wt} \%$ carbon novel method electrodes (c).

When comparing the cyclic voltammograms of all three electrodes in Figure 3.8(a), the novel method electrodes were found to clearly exhibit higher electrode conductivity as compared to the standard method. This apparently stems from the improved microstructure of the novel electrodes as discussed in the previous section. It is interesting to note that the $15 \mathrm{wt} \%$ carbon electrode had the same conductivity as the $7.5 \mathrm{wt} \% \mathrm{C}$ electrode, which proves that the higher conductivity of the new electrode is not due to higher carbon content vis-á-vis the standard electrode but due to its improved microstructure. This means that the removed binder $(7.5 \mathrm{wt} \%)$ can be advantageously replaced with active material for higher energy density.

Galvanostatic charge/discharge tests were also performed to evaluate further the electrochemical properties of the electrodes fabricated using the two different methods. Figure 3.9 shows the second cycle lithiation and delithiation, the discharge 

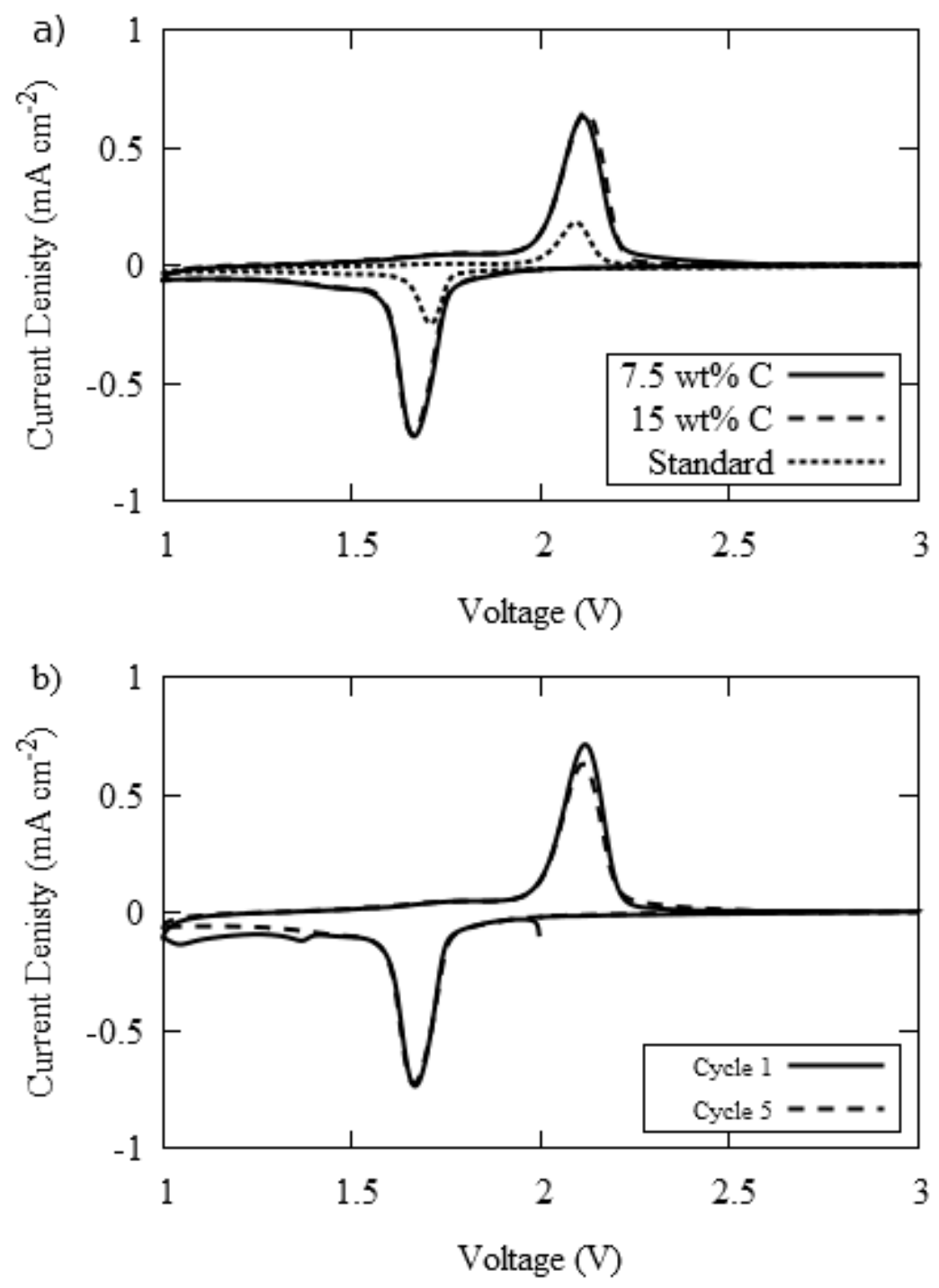

Figure 3.8: Reproducible (second to fifth cycles) cyclic voltammograms for electrodes fabricated using the standard, $7.5 \mathrm{wt} \%$ carbon novel, and 15 wt $\%$ carbon novel methods (a). Differences between the first and fifth cycle CVs for one of the electrodes ( $7.5 \mathrm{wt} \%$ carbon novel method) (b). ${ }^{3}$ 
and charge, respectively. The resulting charge and discharge curves coincide with the data from the cyclic voltammograms that show higher lithiation and delithiation peaks for the novel method electrodes as compared to the standard method.

It is known that during lithiation (refer to reaction 3.1), a phase change occurs from the tetragonal anatase to orthorhombic $\mathrm{Li}_{0.5} \mathrm{TiO}_{2}$ [34]. In general, the discharge (delithiation) curve can be broken into three parts: Region I in Figure 3.9(a) represents the initial insertion of lithium into the anatase lattice for the formation of a solid-solution up to the solubility limit of anatase, corresponding to $x=0.12$ in $\mathrm{Li}_{\mathrm{x}} \mathrm{TiO}_{2}[36,59]$. This dilute Li-anatase was labeled recently $\alpha$-phase by Belak et al. [36]. The voltage plateau region (region II) represents a reversible bi-phasic equilibrium between the lithium-poor anatase and its lithium-rich ordered $\mathrm{Li}_{0.5} \mathrm{TiO}_{2}$ counterpart $[35,36,38]$, otherwise called $\beta$-phase [36]. The longer the plateau, the higher the number (approaching $x=0.5$ ) of Li-ions inserted into the $\mathrm{TiO}_{2}$ lattice, thus a larger charge storage capacity. The tail part of the lithiation curve, up to $\sim 200 \mathrm{~mA} \mathrm{~h} \mathrm{~g}^{-1}$ corresponding to $x \sim 0.6$, according to Belak et al. [36] reflects extra Li storage in vacant sites of the $\beta$-phase.

The charge curve (Figure 3.9(b)) behaves much like the discharge curve, however it acts in reverse. The first region of the charge curve is the surface area dependent slope (region I) corresponding to Li-ion extraction from the Li-poor anatase phase $\left(\mathrm{Li}_{0.12} \mathrm{TiO}_{2}\right)$ [38]. This process happens very quickly as this phase is characterized by high ionic activity [36]. Contrary, the diffusion-controlled region of the charge curve (region II) is where the ions are extracted from the lithium-rich $\mathrm{Li}_{0.5} \mathrm{TiO}_{2}$ phase. Unlike the discharge, the end of the charge curve is asymptotic. This is because a finite number of Li-ions are being reversibly stored in the lattice. It is noteworthy here that Li-ion extraction from the tail zone of the discharge curve (where $x>0.5$ in $\mathrm{Li}_{\mathrm{x}} \mathrm{TiO}_{2}$ ) is not energetically favored [36] and as result cycle life

\footnotetext{
${ }^{3}$ Separation of peaks seen in $\mathrm{CV}$ is associated with the phase change that $\mathrm{TiO}_{2}$ undergoes. In order for the anatase to change from $\alpha-\mathrm{Li}_{\mathrm{x}} \mathrm{TiO}_{2}$ to $\beta-\mathrm{Li}_{\mathrm{x}} \mathrm{TiO}_{2}$ (and vice versa), it must overcome an activation energy at $1.75 \mathrm{~V}$ and $2.1 \mathrm{~V}$ for oxidation and reduction, respectively. Thus, there is an inherent polarization associated with the charge and discharge of anatase.
} 

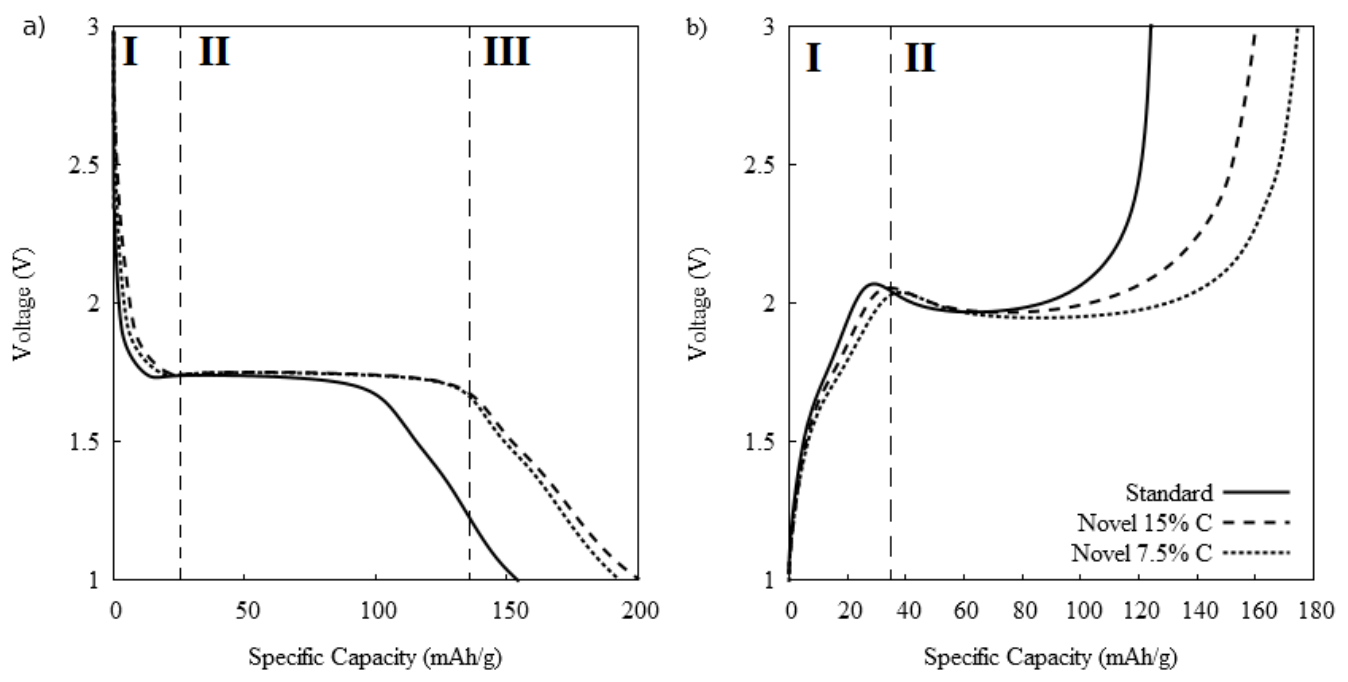

Figure 3.9: Figure 9. Galvanostatic (second cycle) discharge (lithiation) (a) and charge (delithiation) (b) curves for electrodes fabricated using the standard, $7.5 \mathrm{wt} \%$ carbon novel, and $15 \mathrm{wt} \%$ carbon novel methods.

is negatively impacted [29].

Upon examination of the galvanostatic curves in Figure 3.9, it is apparent that the two electrode types exhibited dramatically different charge and discharge behavior despite that both used the same active material. Thus the standard method electrode had charge and discharge capacities of 124 and $154 \mathrm{~mA} \mathrm{~h} \mathrm{~g}^{-1}$, respectively; $154 \mathrm{~mA} \mathrm{~h} \mathrm{~g}^{-1}$ corresponds to $x=0.46$ in $\mathrm{Li}_{\mathrm{x}} \mathrm{TiO}_{2}$. In the meantime, the novel method $7.5 \mathrm{wt} \%$ and $15 \mathrm{wt} \%$ carbon electrodes had charge capacities of 174 and $160 \mathrm{~mA} \mathrm{~h} \mathrm{~g}^{-1}$, while their discharge capacities were 193 and $201 \mathrm{~mA} \mathrm{~h} \mathrm{~g}^{-1}$, corresponding to $x=0.57$ and 0.6 in $\mathrm{Li}_{\mathrm{x}} \mathrm{TiO}_{2}$, respectively. In comparing the surface area-controlled region (region I) of the charge curve, the specific capacities are seen to be almost the same, reflecting the fact that the surface areas of the standard and novel method electrodes are essentially the same (48 versus $50 \mathrm{~m}^{2} \mathrm{~g}^{-1}$ ). It is in the diffusion-controlled region (plateau, region II), where the two electrodes show significant difference in charge capacity. Since both electrodes were built with the 
same active material $\left(\mathrm{P} 25 \mathrm{TiO}_{2}\right)$, their different storage capacities apparently are due to the higher electronic conductivity endowed to the electrode by the novel binder-free method that allowed for higher lithiation and delithiation degrees to be attained. It is known that nanosizing of particles is accompanied by nanoeffects, namely the decrease of diffusion path length [60]. In this case, the particle sizes are identical as the same particles are used in both methods. While the nanosized particles do in fact decrease path length, increasing diffusion rate of ions through the lattice, the novel method electrodes do this more effectively. Two reasons are suggested for the increased conductivity exhibited by the novel method electrodes. The conductivity is higher in the novel method electrodes because of more intimate mixing of the carbon and titania phases. The other contributing factor to attainment of higher conductivity by the novel-built electrodes may be in-situ carbon deposition from the removal of paste ingredients like ethyl cellulose during the sintering process. This, combined with the necking of particles creates a conductive "highway network" along the electrode structure that leads to higher reversibly accessed storage capacities. The performance of the novel-built electrodes becomes even more impressive if it is considered that the P25 active material used in this work was not pure or specially nanoengineered anatase ( $86 \%$ anatase content) in comparison to exquisite anatase nanostructures with similar response reported in the literature $[28,41]$.

But in addition to their superior charge storage properties the novel electrodes yielded drastically better cyclability than the standard method electrode. For this comparison the novel electrode with $7.5 \%$ carbon was selected for testing as it was found earlier (Figure 3.9) to have higher capacity and advantageously contains more active materials hence makes it a better candidate for application. For each of the electrodes, 11 galvanostatic cycles were performed. Figure 3.10 shows the cycling results in terms of charge/discharge capacities and coulombic efficiency for the two electrodes. As it can be seen, the novel $7.5 \mathrm{wt} \%$ electrode had by comparison excellent reversibility. Ignoring the first cycle, the novel electrode had 
a coulombic efficiency between 90 and $94 \%$ compared to $80-82 \%$ for the standard electrode. It is interesting that the coulombic efficiency improved with cycling for the novel electrode from 90 to $94 \%$. But the biggest difference between the two electrodes was in the degree by which the charge and discharge capacities decreased upon cycling. Thus in the case of the novel electrode over the course of the 10 cycles, the charge capacity decreased gradually approaching a constant value at about $92 \%$ retention level. By comparison the charge capacity of the standard electrode had dropped down to only 53\%. It is clear that the novel electrode owing to the better active material/carbon intermixing and inter-particle necking endowed by the controlled sintering provides a superior electrode platform.
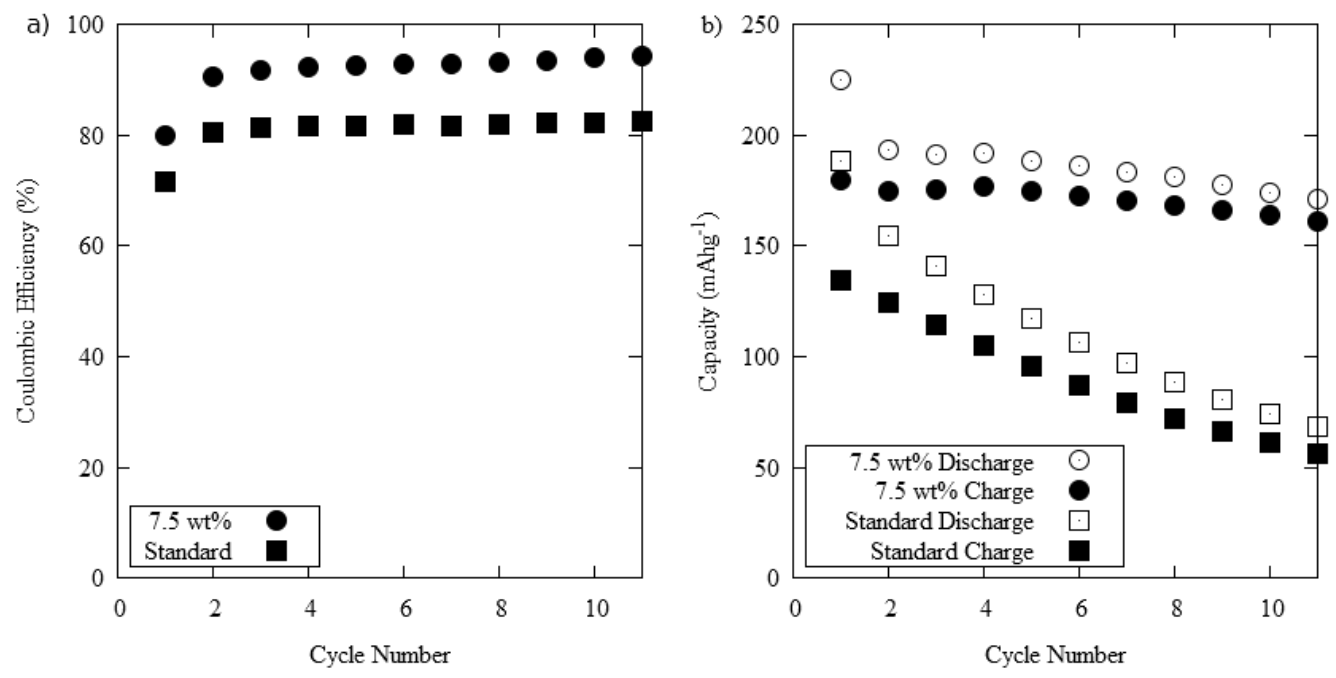

Figure 3.10: Galvanostatic cycling of the standard and novel ( $7.5 \mathrm{wt} \%$ carbon) electrodes: Coulombic efficiencies (a) and charge/discharge capacities (b). ${ }^{4}$

\footnotetext{
${ }^{4} 100$ cycles at $1 \mathrm{C}$ was preformed later, as P25 was fabricated with an aluminum foil substrate.
} This can be seen in Appendix C 


\subsection{Conclusions}

A novel electrode fabrication method has been developed allowing the construction of binder-free electrodes with superior lithium-ion intercalation properties when compared to electrodes prepared by the standard binder-based method. As a model active material, to demonstrate the advantages of the new fabrication process, the commercially available P25 nanoanatase was used. The new fabrication process involves the preparation of an ethanolic $\mathrm{TiO}_{2} /$ carbon paste employing $\alpha$-terpineol and ethyl cellulose as rheological agent and spacer, respectively. The paste, that is suitable for screen printing was shown to endow the fabricated mesoporous electrode with significantly better carbon dispersion due to smaller agglomerate size, resulting in enhanced inter-particle (active-conducting) mixing and packing. Upon deposition of the paste by screen-printing, sintering was performed under air at $450{ }^{\circ} \mathrm{C}$ following a controlled heating profile. Such relatively high temperature sintering treatment was shown to promote inter-particle necking leading to a mechanically robust mesoporous electrode structure that better accommodated strain induced by the lithium-ion insertion and extraction process.

Cyclic voltammetry and galvanostatic charge/discharge testing proved the novel method-built electrodes to exhibit dramatically improved performance over the standard electrodes in terms of conductivity, specific charge capacity, and cyclability. Thus the specific charge (delithiation) capacity of the novel method electrode (92.5 $\mathrm{wt}^{\mathrm{t}} \% \mathrm{TiO}_{2} / 7.5 \mathrm{wt} \% \mathrm{C}$ ) was $174 \mathrm{~mA} \mathrm{~h} \mathrm{~g}^{-1}$ compared to $124 \mathrm{~mA} \mathrm{~h} \mathrm{~g}^{-1}$ for the standard method electrode $\left(85 \mathrm{wt} \% \mathrm{TiO}_{2} / 7.5 \mathrm{wt} \% \mathrm{C} / 7.5 \mathrm{wt} \% \mathrm{PVDF}\right.$ ), representing 103 and $74 \%$ of the theoretical capacity (corresponding to $\mathrm{Li}_{0.5} \mathrm{TiO}_{2}$ ), respectively. At the same time, after 10 cycles, the novel electrode exhibited $90-94 \%$ coulombic efficiency and more than $92 \%$ capacity retention, while the corresponding values for the standard electrode were only $80-82 \%$ and $53 \%$ respectively. The performance of the novel-built electrodes becomes more impressive if it is considered that the P25 active material used in this work was not pure or specially nanoengineered 
anatase ( $86 \%$ anatase content). It is clear that the novel binder-free fabrication method has the potential of building superior electrode platforms in a scalable and cost-effective way.

\subsection{Acknowledgements}

This research is supported with a strategic project grant from the Natural Sciences and Engineering Research Council of Canada (NSERC).

\subsection{References}

[1] J. Goodenough, Y. Kim, Chem. Mater., 22(3), 587 (2010).

[2] D. A. Notter, M. Gauch, R. Widmer, R. Zah, H.-J. Althaus, Environ. Sci. Technol., 44, 6550 (2010).

[3] B. Scrosati, J. Hassoun, Y.-K. Sun, Energ. Environ. Sci., 4, 3287 (2011).

[4] V. Etacheri, R Marom, R. Elazari, G. Salitra, D. Aurbach, Energy Environ. Sci., 4, 3243 (2011).

[5] C. Daniel, JOM-J. Min. Met. Mat. S., 60(9), 43 (2008).

[6] J. Li, C. Daniel, D. Wood, J. Power Sources, 196(5), 2452 (2011).

[7] P. G. Bruce, S. A. Freunberger, L. J. Hardwick, J.-M. Tarascon, Nat. Mater., 11(1), 19 (2012).

[8] K. T. Lee and J. Cho, Nano Today, 6(1), 28 (2011).

[9] L. Cheng, X.-L. Li, H.-J. Liu, H.-M. Xiong, P.-W. Zhang, Y.-Y. Xia, J. Electrochem. Soc., 154, A692 (2007).

[10] G.-N. Zhou, H.-J. Liu, J.-H. Zhuang, C.-X. Wang, Y.-G. Wang, Y.-Y. Xia, Energ. Environ. Sci., 4, 4016 (2011).

[11] B. L. Ellis, K. T. Lee, L. F. Nazar, Chem. Mater, 22(3), 691 (2010). 
[12] J. S. Chen, L. A. Archer, X. W. Lou, J. Mater. Chem., 21(27), 9912 (2011).

[13] R. Marom, S. F. Amalraj, N. Leifer, D. Jacob, D. Aurbach, J. Mater. Chem., 21(27), 9938 (2011).

[14] C. M. Julien, A. Mauger, K. Zaghib, J. Mater. Chem., 21(27), 9955 (2011).

[15] F. M. Courtel, H. Duncan, Y. Abu-Lebdeh, I. J. Davidson, J. Mater. Chem., 21(27), 10206 (2011).

[16] K.-H. Lee and S.-W. Song, ACS Appl. Mater. Interf., 3(9), 3697 (2011).

[17] X. Lou and Y. Zhang, J. Mater. Chem., 21, 4156 (2011).

[18] N. Takami, A. Satoh, M. Hara, T. Ohsaki, J. Electrochem. Soc., 142(2), 371 (1995).

[19] H. Buqa, D. Goers, M. Holzapfel, M. E. Spahr, P. Novak, J. Electrochem. Soc., 152(2), A474 (2005).

[20] M. Fujimoto, Y. Shoji, Y. Kida, R. Ohshita, T Nohma, K. Nishio, J. Power Sources, 72, 226 (1998).

[21] X. Xiao, P. Liu, J. S. Wang, M. W. Verbrugge, M. P. Balogh, Electrochem. Commun., 13, 209 (2011).

[22] G. Wang, X. Shen, J. Yao, and J. Park, Carbon, 47, 2049 (2009).

[23] Y. Hwa, C.-M. Park, H.-J. Sohn, J. Power Sources, 222, 129 (2013).

[24] A. Magasinski, P. Dixon, B. Hertzberg, A. Kvit, J. Ayala, G. Yushin, Nat. Mater., 9, 353 (2010).

[25] G.-N. Zhu, H.-J. Liu, J.-H. Zhuang, C.-X. Wang, Y.-G. Wang, Y.-Y. Xia, Energ. Environ. Sci., 4, 4016 (2011).

[26] A. Laumann, M. Bremholm, P. Hald, M. Holzapfel, K. T. Fehr, B. B. Iverson, J. Electrochem. Soc., 159(2), A166 (2012).

[27] G. Armstrong, A. R. Armstrong, P. G. Bruce, P. Reale, B. Scrosati, Adv. Mater., 18, 2597 (2006). 
[28] J. S. Chen and X. W. Lou, Electrochem. Commun., 11(12), 2332 (2009).

[29] M. Mancini, P. Kubiak, J. Geserick, R. Marassi, N. Husing, M. WohlfahrtMehrens, J. Power Sources, 189, 585 (2009).

[30] S. K. Panda, Y. Yoon, H. S. Jung, W.-S. Yoon, H. Shin, J. Power Sources, 204, 162 (2012).

[31] X. Su, Q.-L. Wu, X. Zhan, J. Wu, S. Wei, Z. Guo, J. Mater. Sci., 47, 2519 (2012).

[32] J. Wang, J. Polleux, J. Lim, B. Dunn, J. Phys. Chem. C, 111, 14925 (2007).

[33] T. Brezesinski, J. Wang, J. Polleux, B. Dunn, S. H. Tolbert, J. Am. Chem. Soc., 131, 1802 (2009).

[34] H. Lindstrom, S. Sodergren, A. Solbrand, H. Rensmo, J. Hjelm, A. Hagfeldt, S.-E. Lindquist, J. Phys. Chem. B, 101(39), 7717 (1997).

[35] M. Wagemaker, W. J. Borghols, F. M. Mulder, J. Am. Chem. Soc., 129(14), 4323 (2007).

[36] A. A. Belak, Y. Wang, A. Van der Ven, Chem. Mater., 24(15), 2894 (2012).

[37] W. J. Borghols, D. Lutzenkirchen-Hecht, U. Haake, E.R. van Eck, F. M. Mulder, M. Wagemaker, Phys. Chem. Chem. Phys., 11, 5742 (2009).

[38] C. Jiang, M. Wei, Z. Qi, T. Kudo, I. Honma, H. Zhou, J. Power Sources, 166, 239 (2007).

[39] M. Zukalova, M. Kalbac, L. Kavan, I. Exnar, M. Grätzel, Chem. Mater, 17, 1248 (2005).

[40] S.-T. Myung, N. Takahashi, S. Komaba, C. S. Yoon, Y.-K. Sun, A. Khalil, H. Yashiro, Adv. Func. Mater, 21, 3231 (2011).

[41] T. Hu, X. Sun, H. Sun, M. Yu, F. Lu, C. Liu, J. Lian, Carbon, 51, 322 (2013).

[42] M. Mancini, F. Nobili, R. Tossici, M. Wolfahrt-Mehrens, R. Marassi, J. Power Sources, 196(22), 9665 (2011). 
[43] A. Moretti, G.-T. Kim, D. Bresser, K. Renger, E. Paillard, R. Marassi, M. Winter, S. Passerini, J. Power Sources, 221, 419 (2013).

[44] S. Ito, P. Chen, P. Comte, M. K. Nazeeruddin, P. Pechy, M. Grätzel, Prog. Photovoltaics, 15, 603 (2007).

[45] C. Charbonneau, K. E. Lee, G.-B. Shan, M. A. Gomez, R. Gauvin, G. P. Demopoulos, Electrochem. Solid St., 13(8), H257 (2010).

[46] L. Kavan, M. Grätzel, J. Rathousky, A. Zukal, J. Electrochem. Soc., 143(2), 394 (1996).

[47] B. Ohanti, O. O. Prieto-Mahaney, D. Li, R. Abe, J. Photoc.Photobio., 216, 179 (2010).

[48] L. N. Lewis, J. L Spivack, S. Gasaway, E. D. Williams, J. Y. Gui, V. Manivannan, O. P. Siclovan, Sol Energy Mater. Sol. Cells, 90, 1041 (2006).

[49] K. E. Lee, C. Charbonneau, G.-B. Shan, G. P. Demopoulos, R. Gauvin, JOMJ. Min. Met. Mat. S., 61(4), 52 (2009).

[50] P. W. Trimby, Ultramicroscopy, 120, 16 (2012).

[51] R. R. Keller and R. H. Geiss, J. Microsc., 245, 245 (2012).

[52] N. Brodusch, H. Demers, R. Gauvin, J. Microsc., 250, 1 (2013)

[53] S. Zaefferer, Ultramicroscopy, 107, 254 (2007).

[54] S. I. Wright, in Electron Backscatter Diffraction in Materials Science, A. J. Schwartz, M. Kumar, B. L. Adams, Editors, p 51 Plenum, New York (2000).

[55] M. Koudriachova, N. Harrison, S. de Leeuw, Phys. Rev. B, 65(23), 1 (2002).

[56] D. Deng, M. Kim, J. Lee, J. Cho, Energ. Environ. Sci., 2, 818 (2009).

[57] M. Sussman, N. Brodusch, R. Gauvin, G. P. Demopoulos, ECS Trans., 50(24), 37 (2013).

[58] R. van de Krol, A. Goossens, E. Meulenkamp, J. Electrochem. Soc., 146(9), 3150 (1999). 
[59] Y. Ma, G. Ji, B. Ding, J. Y. Lee, J. Mater Chem., 22, 24380 (2012).

[60] C. Jiang, E Hosono, H. Zhou, Nano Today, 1(4), 28 (2006). 


\section{Chapter 4}

\section{Mesoporous Brookite Nanoplatelets with Superior Lithium-ion Intercalation Stability}

In chapter 3, it was seen that by removing non-electroactive binders and using a chemo-mechanical fabrication protocol, the reversible capacity of $\mathrm{TiO}_{2}$ was increased significantly. As well, it has been proven that 2-D and mesoporous structures can increase the reversible capacity of electrode materials. In this chapter the focus shifts to the characterization of mesoporous brookite nanoplatelets produced using aqueous solution synthesis. Using electrochemical and diffraction techniques, the lithiation mechanism of brookite is examined, and the effect on the crystal structure is determined. Moreover, the unique structure lends itself to higher capacities than its nanoparticle predecessors, and has excellent capacity retention and stability even at high rate (previously published in: M. Sussman, M. Celikin, A. Yasin, G. P. Demopoulos, Mesoporous Brookite Nanoplatelets with Superior Lithium-ion Intercalation Stability, Electrochimica Acta, 138, (2014) 215-223).

\subsection{Abstract}

Nanoscale $\mathrm{TiO}_{2}$ attracts a lot of research interest as a Li-ion intercalation material. By far anatase in different nanostructures including nanosheets and mesoporous particles has been the center of attention. In this work the Li-ion intercalation behavior of another less explored nanoscale $\mathrm{TiO}_{2}$ phase, brookite, is studied. In particular the study focuses on a unique mesoporous nanoplatelet structured brookite material synthesized via a novel aqueous solution process. These unique meso- 
porous single crystal brookite nanoplatelets ( $\mathrm{mNPl} ; 135 \mathrm{~m}^{2} \mathrm{~g}^{-1}$ surface area with $\sim 5-10 \times 20 \times 50 \mathrm{~nm}$ dimensions and $2-8 \mathrm{~nm}$ pore size) provide both favorable large electrolyte/material interface and short Li-ion diffusion length, important considerations for good Li-ion intercalation performance. A study of the mechanism of lithiation using electrochemical impedance spectroscopy at different states of discharging and X-ray diffraction of the lithiated and de-lithiated mNP1 brookite is reported along cycling data at different rates. It is shown that a solid-solution forms upon lithiation corresponding to orthorhombic $\mathrm{Li}_{0.5} \mathrm{TiO}_{2}$ that is isostructural with brookite. The brookite nanoplatelets were found to progressively undergo crystal structure enhancement upon cycling despite certain lattice distortion occurring during lithiation that allows them to quickly relax upon delithiation in an extremely efficient ( $>99.7 \%$ coulombic efficiency) reversible manner translating to excellent Li-ion intercalation stability.

\subsection{Introduction}

Titania $\left(\mathrm{TiO}_{2}\right)$ has been studied extensively for its lithium intercalation properties $[1,2]$. While much of the focus has been on anatase [3--5], other naturally occurring [6--8] and synthetic phases [9--11] of $\mathrm{TiO}_{2}$ have been investigated.

While all phases of $\mathrm{TiO}_{2}$ lithiate as follows:

$$
\mathrm{Ti}^{\mathrm{IV}} \mathrm{O}_{2}+\mathrm{x}\left(\mathrm{Li}^{+}+\mathrm{e}^{-}\right) \rightleftharpoons \mathrm{Li}_{\mathrm{x}} \mathrm{Ti}_{1-\mathrm{x}}^{\mathrm{IV}} \mathrm{Ti}_{\mathrm{x}}^{\mathrm{III}} \mathrm{O}_{2}
$$

anatase attracts much interest as it can easily intercalate lithium in both the bulk $(\geq 120 \mathrm{~nm})[12]$ and nano scales $[13,14]$. Anatase can theoretically intercalate $x=0.5 \mathrm{Li}$ in $\mathrm{Li}_{\mathrm{x}} \mathrm{TiO}_{2}$ (approximately $168 \mathrm{~mA} \mathrm{~h}^{-1}$ ) in the bulk, and $x \geq 0.5$ at the nanoscale [15]. Anatase lithiates in several stages[16, 17]: 1) solid solution of $\mathrm{TiO}_{2}$ and $\mathrm{Li}$ forming $\left.\alpha-\mathrm{Li}_{0.1} \mathrm{TiO}_{2} ; 2\right)$ biphasic $\left(\mathrm{TiO}_{2}-\mathrm{Li}_{\mathrm{x}} \mathrm{TiO}_{2}\right)$ diffusion where the lithiated $\mathrm{TiO}_{2}$ incurs a transition from $\alpha-\mathrm{Li}_{0.1} \mathrm{TiO}_{2}$ to $\left.\beta-\mathrm{Li}_{0.5} \mathrm{TiO}_{2} ; 3\right)$ lithiation into 
octahedral sites of stable $\beta-\mathrm{Li}_{\mathrm{x}} \mathrm{TiO}_{2}$ from $x=0.5$ to $x=0.58$ [17]; 4) a second biphasic diffusion above $x=0.6$ where $\beta-\mathrm{Li}_{\mathrm{x}} \mathrm{TiO}_{2}$ coexists with $\gamma-\mathrm{Li}_{1} \mathrm{TiO}_{2}$ until 1 $\mathrm{Li}$ is stored within the $\mathrm{TiO}_{2}$ lattice. While the last bi-phasic stage can lead to full lithiation, this level of charge storage---due to poor ion conductivity of $\mathrm{LiTiO}_{2}$ as discussed by Belak, et al.---cannot in general be materialized [17].

Nanosizing $[12,16,18]$ and in particular nanostructuring in the form of mesoporous spheres comprising 6-8 $\mathrm{nm}$ anatase crystallites [19], nanowire networks [20] or 2-D structures (nanosheets or nanoplatelets) $[17,21]$ have provided avenues for achieving high reversible storage capacity at good rates with anatase. In addition to the naturally occuring tetragonal anatase phase, the synthetic $\mathrm{TiO}_{2}-\mathrm{B}$ (bronze) phase, which is monoclinic, also attracts a lot of research attention as it is shown, in particular in the form of nanosheets, [22] to be superior in terms of rate capability due to its open structure.[23] However, the large scale synthesis of $\mathrm{TiO}_{2}-\mathrm{B}$ is rather difficult to achieve because of the complex solvothermal process employed. Rutile, in the meantime suffers in terms of poor Li-ion diffusion through tetrahedral vacancies [24] and complex phase transition sequence during lithiation $[18,25]$.Nanoneedles, extremely high surface area nanoparticles, and nanoporous crystals have shown promise for lithiation. [26--28] However, as with $\mathrm{TiO}_{2}$-B, they are difficult to synthesize in large scale because of complex synthesis techniques.

Beyond anatase, $\mathrm{TiO}_{2}-\mathrm{B}$ and rutile, less is known about the Li-intercalation properties of the brookite polymorph. This is owed to the difficulty in producing this phase and partly because in the bulk state it has proven to be a poor intercalation compound [29]. Nanoscale brookite, however is interesting to study as some recent reports provide evidence of potentially attractive Li-ion battery relevant properties. For one, brookite is an orthorhombic crystal that differs from the tetragonal anatase and rutile or the monoclinic bronze phase [30]. Its discharge curves are rather slanted without a plateau region indicative of a different intercalation mechanism than that of anatase. Further, nanostructuring has not been explored to the same extent, especially outside of nanoparticle sizing [31]. 
Reddy, et al. [7] were the first to investigate brookite as an electrode material. They synthesized nanocrystalline brookite via thermolysis of highly acidic (3M $\mathrm{HCl}) \mathrm{TiCl}_{4(\mathrm{aq})}$ at $100^{\circ} \mathrm{C}$ for 48 hours. Their product was a colloidal suspension of rutile and brookite, from which the rutile phase was removed via peptization with $\mathrm{HNO}_{3}$. They were able to achieve a reversible capacity of $170 \mathrm{~mA} \mathrm{~h} \mathrm{~g}^{-1}$ at a rate of $\mathrm{C} / 10$. Further, the voltage profile of the lithiation (discharge) was completely different compared to rutile and anatase. The mechanism postulated was that during the lithiation of brookite the crystal undergoes amorphization (as evidenced by peak broadening seen in ex-situ XRD), then on delithiation partially recovers its crystallinity. Lee, et al. [32] synthesized $10 \mathrm{~nm}$ brookite nanoparticles by dissolving urea in $\mathrm{TiCl}_{3(\mathrm{aq})}$ and refluxing the solution at $100^{\circ} \mathrm{C}$ for 7 hours. They were able to achieve $150 \mathrm{~mA} \mathrm{~h} \mathrm{~g}^{-1}$ but had a large capacity fading even at the very slow rate of $\mathrm{C} / 5$. Reddy, et al. [31] again published work on brookite, focusing this time on the nanoparticle size effect. They determined that brookite's intercalation behavior is more size sensitive than that of anatase. Thus, by comparison reversible capacities were $170 \mathrm{~mA} \mathrm{~h} \mathrm{~g}^{-1}$ versus $35 \mathrm{~mA} \mathrm{~h} \mathrm{~g}^{-1}$ at $\mathrm{C} / 5$ after 40 cycles in the case of 10 and $33 \mathrm{~nm}$ crystallites respectively. However, judging from the slope of the discharge capacity vs. cycle number curves, their brookite nanoparticles did not appear to be stable over the long run.

Dambournet, et al. [33] have recently reported on a novel synthesis method for brookite involving high temperature $\left(300-400^{\circ} \mathrm{C}\right)$ thermal decomposition of a titanium oxalate hydrate $\left(\mathrm{Ti}_{2} \mathrm{O}_{3}\left(\mathrm{H}_{2} \mathrm{O}\right)_{2}\left(\mathrm{C}_{2} \mathrm{O}_{4}\right) \cdot \mathrm{H}_{2} \mathrm{O}\right)$ produced by aqueous precipitation [34]. The resultant micron-sized brookite particles were mesoporous with $255 \mathrm{~m}^{2} \mathrm{~g}^{-1}$ surface area. After the formation cycle the specific capacity was $175 \mathrm{~mA} \mathrm{~h} \mathrm{~g}^{-1}$ (at C/7.5) but dropped to $155 \mathrm{~mA} \mathrm{~h} \mathrm{~g}^{-1}$ after 20 cycles with evidence of further capacity fading at longer number of cycles. In the meantime its capacity decreased down to $55 \mathrm{~mA} \mathrm{~h} \mathrm{~g}^{-1}$ at $10 \mathrm{C}$ rate. In contrast to anatase and rutile, brookite was found to exhibit a slanted (no plateau) galvanostatic voltage profile indicative of a solid solution insertion mechanism.[33] The group later attempted 
to explain the mechanism of lithium insertion using first principles modeling.[35] In contrast to the empirical observations of Reddy, et al. of "amorphization" of lithiated brookite, their modeling suggested that the $\mathrm{TiO}_{2}$ framework maintained connectivity throughout intercalation, with expansions along the alpha and beta axes pointing to a flexible brookite framework that can distort and relax upon lithiation and delithiation, respectively, but no experimental proof was provided. They further proposed, based on their first principles analysis that $\mathrm{Li}$-ions intercalate through a solid solution mechanism with local distortion of the brookite crystal lattice to accommodate lithium in 5-coordinated sites. In their model, Dambounet, et al. [35] assumed the following lithiated composition: $\mathrm{Li}_{0.75} \mathrm{TiO}_{2}$. However, this composition corresponds to $260 \mathrm{~mA} \mathrm{~h} \mathrm{~g}^{-1}$ capacity that is observed only during the formation cycle. Thereafter it is known that up to $1 / 3$ of the capacity is lost. In other words the material upon further cycling exhibits a capacity of $170 \mathrm{~mA} \mathrm{~h} \mathrm{~g}^{-1}$ corresponding to a lithiated composition $\mathrm{Li}_{0.5} \mathrm{TiO}_{2}$ or less. Hence, further work is needed to elucidate the lithiation/de-lithiation mechanism in the reversible capacity region.

At present, in addition to yet unconfirmed experimentally intercalation mechanism, the effects of 1-D or 2-D morphology on lithium intercalation of brookite has not been studied as all of the previous work was performed on nanoparticles or mesoporous micron-sized particles. This may be due to the level of difficutly associated with synthesizing brookite, as opposed to anatase and rutile, because of its known metastability [33]. Recent research in our laboratory focusing on forced hydrolysis of aqueous $\mathrm{TiCl}_{4}$ solution[36] has led to the discovery that metastable brookite in the form of nanoplatelets can be produced and recovered via a fast low temperature reaction before appreciable conversion to rutile takes place [37]. 2-dimensional nanostructures have become ideal for providing a platform for facile lithiation at high rate versus the core-shell model of spherical nanoparticles [17, 21, 22, 38, 39]. More specifically, for $\mathrm{TiO}_{2}$, anatase nanosheets have been extensively studied for their rate capability, cyclability, and capacity retention[21, 39]. It is there- 
fore the goal of this work to report on the lithiation/delithiation properties of a new crop of nano- $\mathrm{TiO}_{2}$ materials comprising unique single brookite crystal mesoporous nanoplatelets, elucidate their intercalation mechanism, and demonstrate their superior stability.

\subsection{Experimental}

\subsubsection{Synthesis and Characterization}

Brookite nanoplatelets were synthesized using a novel continuous aqueous process developed in our laboratory[37] upon modification of an earlier batch method developed by Charbonneau, et al.[36]. This process uses forced hydrolysis of $\mathrm{TiCl}_{4}$ to produce $\mathrm{TiO}_{2}$ based on the following reaction:

$$
\mathrm{TiCl}_{4}+\mathrm{H}_{2} \mathrm{O} \longleftrightarrow \mathrm{TiO}_{2}+\mathrm{HCl}
$$

In a typical synthesis run, ${ }^{1}$ an $0.1 \mathrm{M} \mathrm{TiCl}_{4(\mathrm{aq})}$ solution was continuously pumped into a stirred reactor preheated to $80^{\circ} \mathrm{C}$. After the attainment of steady-state operation at 1 hour mean residence time a colloidal nanobrookite suspension was produced. The outflow was collected and treated with $\mathrm{NH}_{4} \mathrm{OH}$ to $\mathrm{pH}=3$ to promote colloid aggregation, an important step in its recovery via centrifugation. The material was washed 3 times with DI water and once with anhydrous ethanol, then dried at $80^{\circ} \mathrm{C}$ overnight. The resulting solid was mechanically ground prior to its use and characterization.

As-prepared samples were characterized by X-ray Diffraction (XRD), performed with a Philips PW1710/00 Diffractometer equipped with a curved graphite monochromator and a copper long fine focus radiation source $(2200 \mathrm{~W}, 60 \mathrm{kV} \max , \lambda \mathrm{CuK} \alpha 1$ $=0.15406 \mathrm{~nm}$ ) operated at $40 \mathrm{~mA}$ and $20 \mathrm{kV}$. Phase composition was determined using Reitveld refinement. Furthermore, electrodes before and after lithiation and

\footnotetext{
${ }^{1}$ Approximate yield of forced hydrolysis reaction is $85 \%$.
} 
delithiation were examined using XRD. The electrodes were removed from the cells, washed in dimethyl carbonate (DMC) and dried under argon. They were then mounted to glass microscope slides with carbon tape. Brunauer-Emmett-Teller (BET) surface area measurements involving $\mathrm{N}_{2}$ adsorption were used to determine specific surface area, pore size distribution, and equivalent dense particle size using a Micromeritics TriStar 3000. All samples weighed approximately $0.1 \mathrm{~g}$ and were degassed for 24 hours at $80^{\circ} \mathrm{C}$ prior to nitrogen physisorption. A Philips CM200 Transmission Electron Microscope (TEM) was used at 200kV to determine particle morphology and crystal structure, as well as to verify crystallinity. TEM specimens were prepared by depositing particles on $\mathrm{Cu}$-grid. Selected-Area Electron Diffraction (SAED) for crystal structure analysis was conducted using a double-tilt specimen holder ( 2 different rotation axes with a maximum tilt angle of $\sim 20$ degrees).

\subsubsection{Electrochemical Measurements}

Electrochemical testing was performed with a Biologic VSP Potentiostat/Galvanostat/EIS system using Swagelok-type cells assembled in an argon-filled glovebox. The synthesized nanobrookite was the active material for the working electrodes, while lithium foil was used for the counter electrode. Electrodes were fabricated using $80 \%$ active material, $10 \%$ acetylene black, and $10 \%$ poly(vinylidene) fluoride (PVDF, HSV 900, Solvay) binder dissolved in 1-methyl 2-pyrrolidone (NMP, Sigma Aldrich) solvent. The resulting slurry was then doctor bladed onto aluminum foil and dried at $120^{\circ} \mathrm{C}$ for 24 hours. The electrodes were then pressed for 5 minutes at $6 \mathrm{t} \mathrm{cm}^{-2}$. The electrolyte used in this work was a solution of $1 \mathrm{M}$ $\mathrm{LiPF}_{6}$ in EC:DMC:DEC (2:2:1 by weight) mixed solvent (BASF).

Galvanostatic charge/discharge testing was performed on the half-cells between 3 and $1.2 \mathrm{~V}$. A specific current of $16.8 \mathrm{~mA} \mathrm{~g}^{-1}$ (corresponding to $\mathrm{C} / 10$ rate) was used for both charging and discharging. Cells were first discharged to insert Li-ions 
into pristine $\mathrm{TiO}_{2}$, and then charged. This testing was carried out over 50 cycles, at which time the cells were charged at $\mathrm{C} / 5, \mathrm{C} / 2,1 \mathrm{C}, 2 \mathrm{C}$, and $10 \mathrm{C}$ rates. Each of these subsequent rates were performed for 5 cycles until 10C which was performed for 10 cycles to observe the effects of charge retention at high rate. Electrochemical Impedence Spectroscopy (EIS) was performed at various points within the first lithiation of the electrode. EIS was performed over the frequency range of 100,000 to $0.01 \mathrm{~Hz}$ and the a.c. modulation was controlled at $5 \mathrm{mV}$. The cells were lithiated to a cut-off voltage (e.g. $2.1,1.8,1.725 \mathrm{~V}$ vs. $\mathrm{Li}^{-} \mathrm{Li}^{+}$), and EIS was performed substituting the cut-off voltage for the open-circuit potential.

\subsection{Results and Discussion}

\subsubsection{Material Characterization}

Figure 4.1 shows the diffraction pattern of the synthesized brookite. The pattern shows the main peak at $2 \theta \approx 25.3^{\circ}$, which is in good agreement with the peak for the (120) plane of brookite. By zooming-in and de-convolution (see Figure D. 1 in the ESI section in Appendix A) it becomes evident that next to the peak at $2 \theta=25.3^{\circ}$ is a second peak seen at $2 \theta=25.7^{\circ}$ that corresponds to brookite's [111] plane. Because these peaks are so close together, it is believed to have created problems in the past with the characterization of the changes the brookite structure undergoes during lithiation---an issue revisited later in this work. Thus in an earlier study[7] that first observed the broadening of brookite's main peaks as a result of lithium insertion, they attributed the broadening specifically to the peak at $2 \theta=25.3^{\circ}$ instead to both, $2 \theta=25.3^{\circ}$ and $25.7^{\circ}$. While the broadening was slight for each of the two adjacent peaks, the combined broadening seen by the convoluted peak couple caused the pattern to look as though brookite was becoming amorphous.

In addition to the characteristic brookite peaks, the XRD pattern of Figure 4.1 shows peaks belonging to rutile. As mentioned earlier brookite is metastable,[40] 


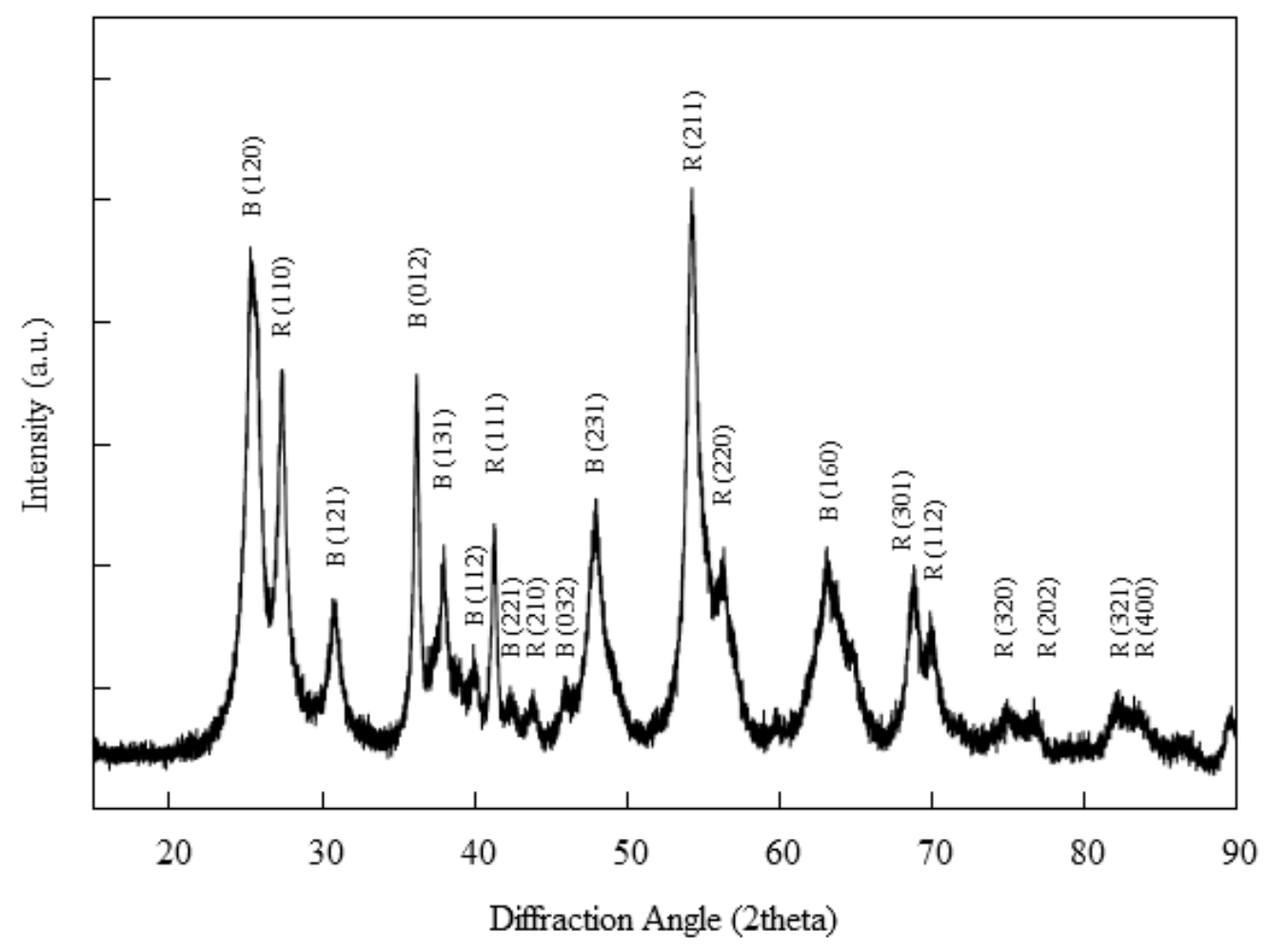

Figure 4.1: X-ray diffraction pattern for aqueous synthesized brookite material.

and if left long enough in the acidic chloride sythnesis solution it starts converting to rutile[37] hence the appearance of rutile. This means that the aqueous solution synthesized brookite is not phase-pure. Via Reitveld refinement (see Figure D.1) it was determined that the composition of the active material was $72.65 \%$ brookite and $27.35 \%$ rutile.

The presence of rutile raises the question of whether the two $\mathrm{TiO}_{2}$ phases (brookite and rutile) are present as individual (phase pure) crystallites or as mixedphase crystallites. To answer this question TEM combined with SAED analysis was undertaken (Figure 4.2). According to the low magnification image in Fig 4.2a the $\mathrm{TiO}_{2}$ material is made up of aggregates approximately 100 to $170 \mathrm{~nm}$ in size. 

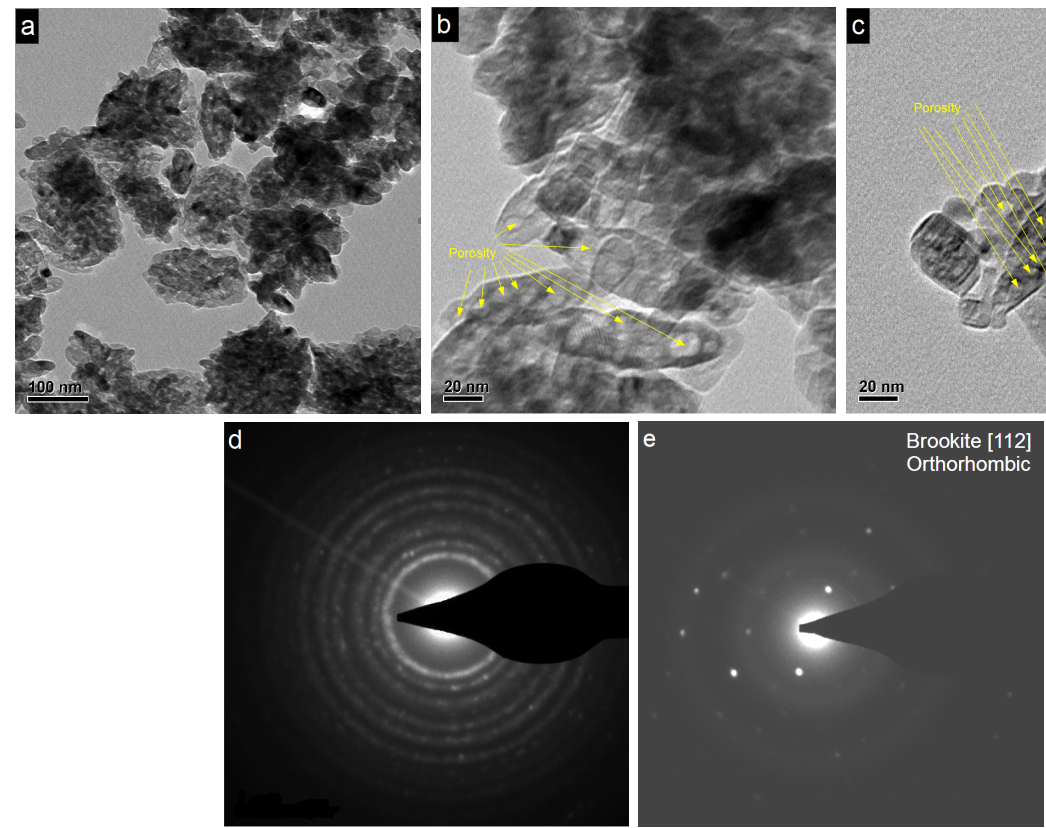

Figure 4.2: Transmission electron micrographs of brookite nanoplatelets. Low magnification of aggregated nanoplatelets (a), high magnification of large aggregated nanoplatelets (b) and high magnification of a small cluster of nanoplatelets (c). SAED of (d) Low magnification aggregate (a) and (e) high magnification clustered platelets (c).

SAED was performed on the entire area of Figure 4.2a for phase identification purposes. Figure $4.2 \mathrm{~d}$ shows the SAED pattern of the aggregate area. The pattern shows rings associated with a polycrystalline solid, and analysis of the pattern determined that the primary phase is brookite. When the aggregates are viewed at higher magnification (Figure 4.2b), individual particles can be seen. Moreover, the higher magnification image shows the true morphology of the particles. The particles are nanoplatelets with 20 to $50 \mathrm{~nm}$ dimensions. The 2-dimensional platelet morphology is confirmed in Figure 4.2c, where overlapping particle boundaries are projected within the individual particles. The platelets are thin enough $(\leq 10 \mathrm{~nm})$ to allow for electrons to pass through them to show the shape of the particle below 
it. The SAED for this small cluster (Figure 4.2e) shows that the nanoplatelets are single brookite crystals. The minor rutile presence, as separate particles, may be explained by the known brookite to rutile transition,[40] and the fact that a small portion of particles spends longer time within the continuous reactor than the mean residence time.[41, 42]

Upon closer examination of the brookite nanoplatelets (Figure $4.2 \mathrm{~b}$ and $\mathrm{c}$ ) they are seen to be porous with pores in the range of few $\mathrm{nm}$ (1-10) in size. The morphological evidence of mesoporous nanoplatelets has been confirmed by $\mathrm{N}_{2}$ adsorption. Figure D.2 shows a typical type II isotherm,[43] which is consistent with a mesoporous nanosized solid. The average pore size was $5.4 \mathrm{~nm}$, which is consistent with the pores seen in the TEM images. The pore size (measured using BET) ranged from 1.8 to $8.3 \mathrm{~nm}$ which is within the mesoporous range (2 to 50 $\mathrm{nm})$. The mesoporosity of the nanoplatelets, as well as their aspect ratio resulted in a high surface area $\left(135 \mathrm{~m}^{2} \mathrm{~g}^{-1}\right.$ as calculated by the BET method). Based on the surface area, the equivalent dense particle size, assuming spherical particles, is $10.5 \mathrm{~nm}$.

As per above data, the produced material is made of individual phase-pure nanoplatelets with the bulk of it (73\%) being brookite and the rest rutile. Moreover the single crystal brookite nanplatelets are found to be porous, a truly unique property that recently was described for other $\mathrm{TiO}_{2}$ crystals, namely anatase.[44, 45] But in contrast to previous synthesis protocols based on template crystal growth,[45] here the mesoporous single crystals of brookite are made via a simple solution synthesis method. The origin of pore formation in single crystals is an open scientific question.[44] For the present system this may have arisen as result of the transformation mechanism via dissolution and recrystallization of the metastable brookite to stable rutile. One thing is certain that the mesoporous brookite nanoplatelets constitute an attractive active material as they provide both favorable large electrolyte/material interface and short Li-ion diffusion length, properties that are characterized in the following section. 


\subsubsection{Li-Ion Intercalation Behavior}

\subsubsection{Galvanostatic Profiles.}

Figure 4.3 shows the voltage profile of galvanostatic charge and discharge of the mixed-phase brookite (73\%)-rutile (27\%) titania as compared to P25 nanoparticles (commercially available mixed-phase anatase (86\%)-rutile $(14 \%)[46,47]) .^{2}$ The discharge (2nd cycle) capacity of the mixed-phase brookite-rutile nanoplatelet $\mathrm{TiO}_{2}$ material (achieved at $\mathrm{C} / 10$ rate) is $135 \mathrm{~mA} \mathrm{~h} \mathrm{~g}^{-1}$, slightly less than that of the mixed phase (anatase-rutile) P25 nanoparticle ( $\sim 30 \mathrm{~nm}$ size). However, these capacity values are based on the total mass of each material that includes rutile in addition to brookite or anatase. It can be assumed that the rutile in either material will not be measurably lithiated as its characteristic plateau at $1.4 \mathrm{~V}$ observed during the formation cycle was not present (see Figure D.3).[25] Further, it is known that nanorutile undergoes phase transformation during cycling resulting in a change in its XRD fingerprint $[18,27]$ not observed in the present work (Fig 5) not to mention that its lithiation is limited at room temperature in larger size crystals. Thus recalculating the discharge capacity on the basis of the brookite active component only (73\%) gives approximately $183 \mathrm{~mA} \mathrm{~h} \mathrm{~g}^{-1}$ slightly over the theoretical value of $168 \mathrm{~mA} \mathrm{~h} \mathrm{~g}^{-1}$ corresponding to $\mathrm{Li}_{0.5} \mathrm{TiO}_{2}$. The slight excess may be taken to reflect the marginal contribution of rutile or simply non-faradaic surface charging. By comparison the corresponding capacity for the active anatase component of $\mathrm{P} 25$ was $160 \mathrm{~mA} \mathrm{~h} \mathrm{~g}^{-1}$.

The lithiation curve of anatase has a plateau at $1.75 \mathrm{~V}$, corresponding to the biphasic diffusion front discussed previously.[47] The lithiation/delithiation curves for brookite, however do not have this plateau, but instead are slanted. Such behavior is characteristic of solid solution mechanism.[33, 35] During lithiation the octahedral interstitial sites expand in the $a$ - and $b$-direction in order to accommodate the insertion of lithium.[35] As the amount of inserted lithium increases, Li-ion dif-

\footnotetext{
${ }^{2}$ In this analysis, rutile is considered inactive in both P25 and mixed-phase brookite.
} 
fusion progressively slows down resulting in lower attained capacity in comparison to the anatase material. On the delithiation side, the curve is indicative of crystal relaxation as the lithium exits the lattice.[35] For P25, a second plateau is seen at $2 \mathrm{~V}$ preceeded by a sharp "hump" that begins at approximately $30 \mathrm{mAhg}-1$. This plateau is associated bi-phasic diffusion of Li-ions out of the crystal structure, and the hump is associated with the activation energy required for the phase change to occur. Another difference between the two materials is their degree of polarization. Thus because there is no phase transition associated with brookite, the polarization of brookite is less than that of the bi-phasic lithiation of P25 (anatase). This polarization difference may in part explain the good capacity retention exhibited by brookite in previous studies.[7, 35]

\subsubsection{Electrochemical Impedance Spectroscopy.}

In order to further understand the lithiation mechanism of brookite, electrochemical impedance spectroscopy (EIS) was performed at various degrees of lithiation during the formation cycle (Figure 4.4a) using a frequency range of $100 \mathrm{MHz}$ to $0.01 \mathrm{~Hz}$. The full set of EIS data in the form of Nyquist plots, the equivalent circuits, and key parameters are reported in the SI part of the paper (Figure D.4, Figure D.6 and Table D.1). During lithiation, the Nyquist plots maintained a constant shape (depicted schematically in Figure 4.4b) representative of a simple Randle's circuit. By reviewing the collected data (Table D.1) and examining the Nyquist plots (Figure D.4) the following observations can be made. First the electrolyte resistance feature, $R_{e}$ (first intercept on the $Z^{\prime}$ axis at the high-frequency end) remains essentially constant throughout the lithiation process as would be expected in the absence of an interfacial decomposition reaction. However the other parameters, namely charge transfer resistance, $R_{c t}$, the double layer capacitance, $C_{d l}$, and the Warburg impedance, $Z_{W}$, were found to vary during the lithiation process. In the case of $R_{c t}$ and $C_{d l}$, variation during lithiation is in direct correlation to each other. $R_{c t}$ 


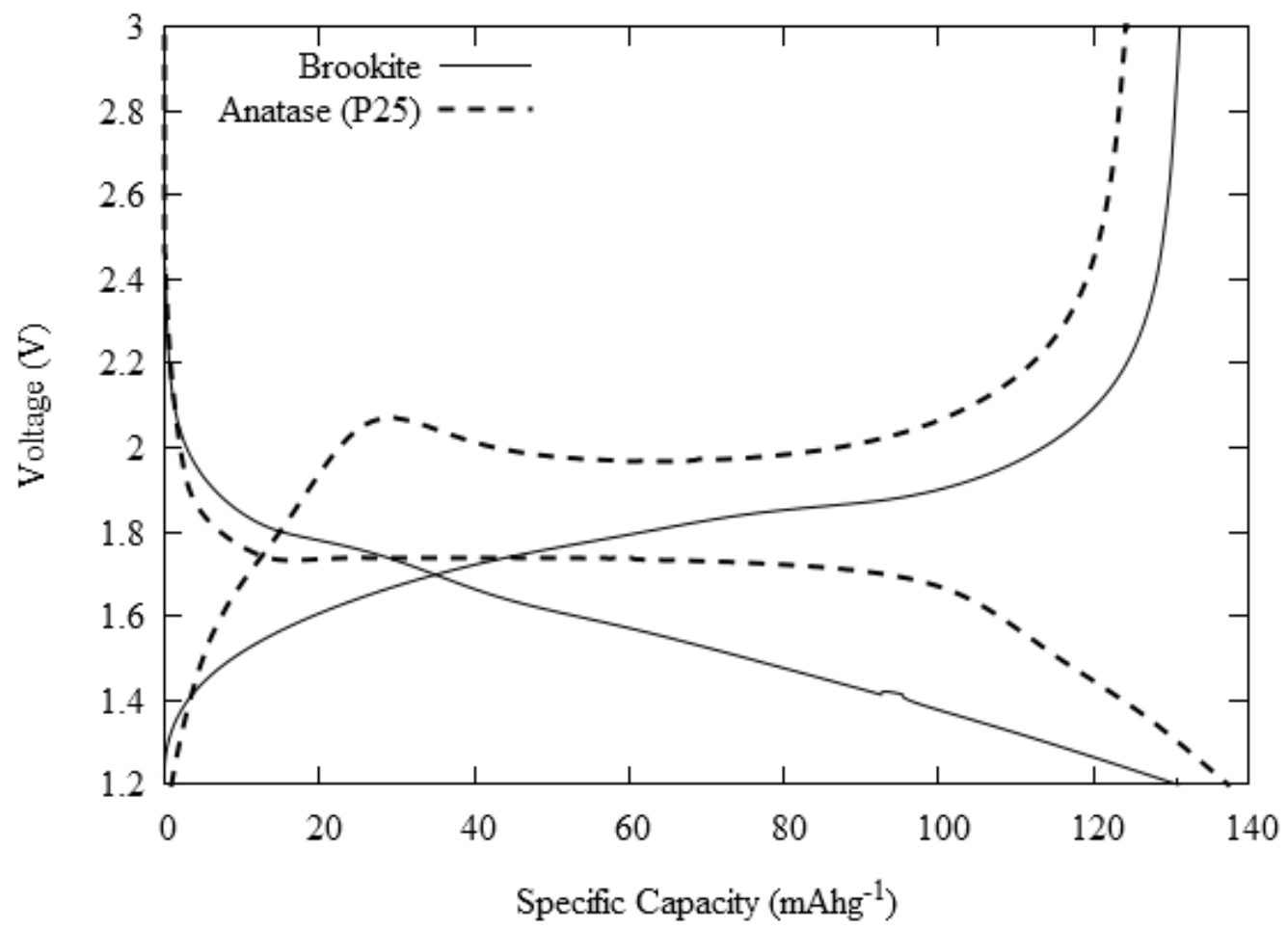

Figure 4.3: Voltage profiles of the 2nd cycle lithiation and delithiation of brookite (solid line) and P25 anatase (dashed line). The capacities of the brookite and $\mathrm{P} 25$ are based on the total amount of $\mathrm{TiO}_{2}$ in the electrode. This amount includes the rutile component, which does not lithiate.

and $C_{d l}$ see an initial increase as the voltage drops from $3 \mathrm{~V}$ to $2.4 \mathrm{~V}$, then reach a minimum at $1.8 \mathrm{~V}$ with $R_{c t}$ showing the biggest decline. Between $3 \mathrm{~V}$ and 2.4 $\mathrm{V}$, there is a negligible amount of lithium that has entered the lattice (Figure 4a). As such, the initial increase apparently relates to the start of migration of Li-ions toward the working electrode where they align on the surface forming a double layer, thus the capacitance increase. The initial increase apparently relates to the start of migration of Li-ions toward the working electrode where they align on the surface forming a double layer, thus the capacitance increase. At the same 
time, the electrons must overcome the low conductivity of the $\mathrm{TiO}_{2}$ host before they can cause the reduction of $\mathrm{Ti}^{4+}$ to $\mathrm{Ti}^{3+}$. This leads to the initial increase in charge transfer resistance. When the voltage has dropped past $2.4 \mathrm{~V}$, the reduction of $\mathrm{Ti}^{4+}$ to $\mathrm{Ti}^{3+}$ begins, leading to a decrease in $R_{c t}$. In other words, the decrease of both $R_{c t}$ and $C_{d l}$ indicates the initiation of lithium intercalation into the brookite lattice. This is seen electrochemically by the initiation of significant increases in capacity seen in Figure 4a. Just as $R_{c t}$ decreases, when the electrons reduce $\mathrm{Ti}^{4+}$ to $\mathrm{Ti}^{3+}$, the entrance of ions into the lattice causes $C_{d l}$ to decrease and remain realtively constant until nearly full lithiation happens. At the same time, $R_{c t}$ is seen to increase as the voltage drops below $1.8 \mathrm{~V}$. This increase most likely arises from a slow down in Li-ion diffusion as ions progressively fill up the host sites, not to mention the compounding effect of resistance due to $\mathrm{TiO}_{2}$ lattice stretching induced by the insertion of lithium as further discussed later. The voltage profile shows a decrease in slope into the linear portion of the curve. This is indicative of the ions inserting steadily into the lattice, rather than the steeply sloping line of surface storage above $2 \mathrm{~V}$.

Considering now $Z_{W}$ that represents Li-ion diffusion resistance (refer to Figure D.4 in the SI section of the paper), we note that initially ( $3 \mathrm{~V}$, i.e. zero state of charge) the diffusion tail is very long and almost vertical (low frequency response), a characteristic of electrode blocking behavior. This behavior means that Li-ions at this stage remain at the surface of $\mathrm{TiO}_{2}$ not yet engaged in charge transfer.[48] With the gradual drop in voltage however the slope of the linear tail part (low frequency range) (Figure D.5) tends to decrease towards $45^{\circ}$ (see data in Table D.1, indicating that the diffusion of $\mathrm{Li}$-ions into the lattice accelerates and the rate limiting step shifts progressively to charge transfer in correspondence to the equivalent circuit of Figure D.6 that has $Z_{W}$ in series with $R_{c} t$. As, however, the voltage drops below $1.5 \mathrm{~V}$ (Figure D.4), not only does $R_{c} t$ increase (Figure $4.4 \mathrm{c}$ and Table D.1) but also the Warburg impedance $Z_{W}$ increases (length of tail part in the low frequency range). As interstitial sites are filled with lithium ions, the driving force for diffu- 

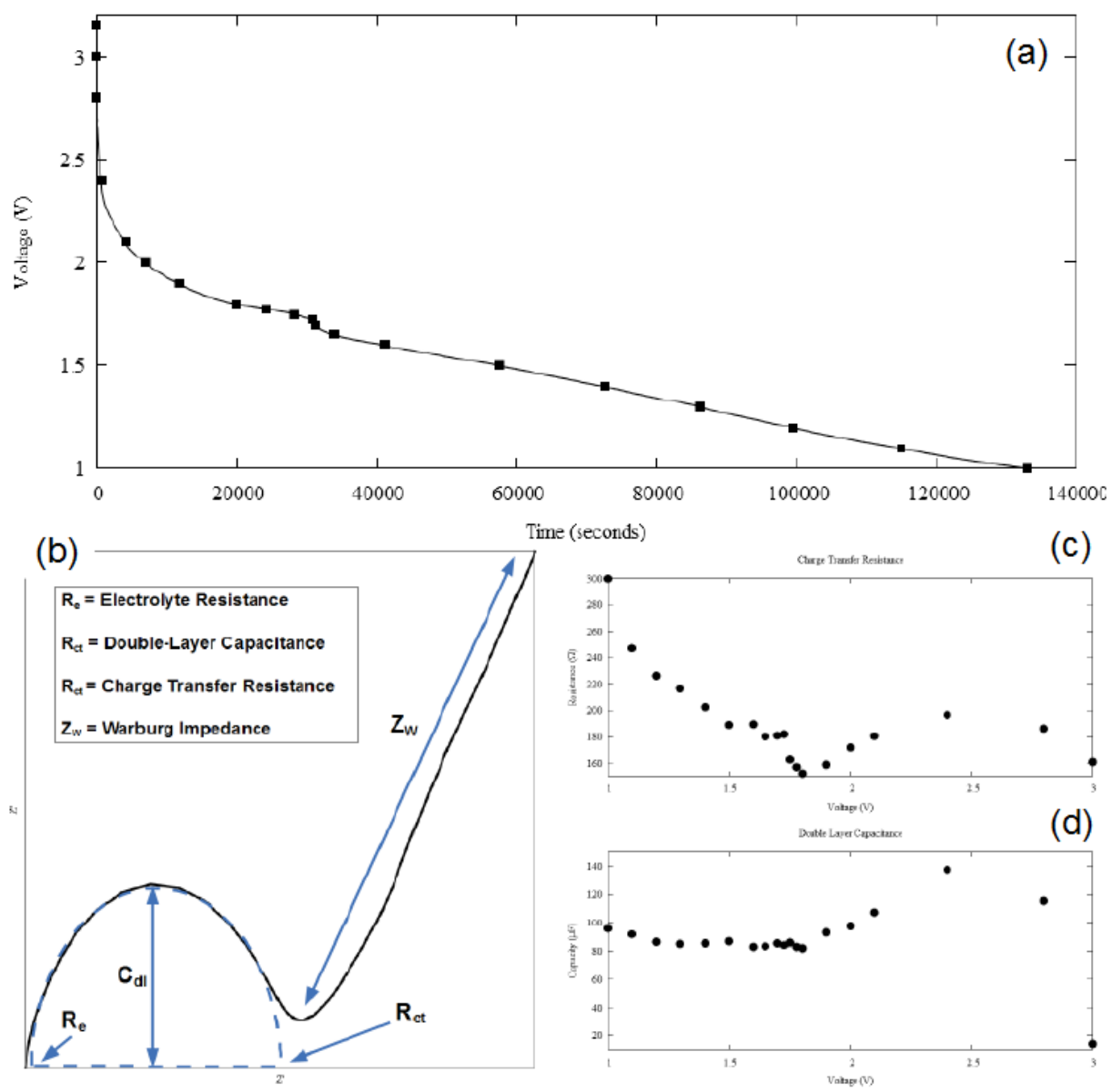

Figure 4.4: Voltage profile of first lithiation of brookite nanoplatelets over 36 hours (a), Representative schematic of Nyquist plot during lithiation (b), trends in charge transfer resistance $R_{c t}$ (c) and double layer capacitance $C_{d l}(\mathrm{~d})$ during the voltage drop from $3 \mathrm{~V}$ to $1 \mathrm{~V} .^{3}$ 
sion decreases. The rate of lithiation, however, remains constant (as the profile is linear, rather than asymptotic as seen in other phases of $\mathrm{TiO} 2$ when lithium can no longer diffuse [48]). The linear profile, in conjunction with the increase in Warburg impedance, indicates that there is a force acting against the continued insertion of lithium. Thus, as interstitial sites are filled with lithium ions, the driving force for diffusion decreases. As well, $\mathrm{Li}^{+}-\mathrm{Li}^{+}$repulsive interactions,[22] increase resistance to intercalation into the lattice, contributing to $\mathrm{Li}$-ion diffusion slow down.

The shape of the delithiation curve (Figure 4.3) shows that the crystal relaxes as the lithium exits the lattice.[35] This is an important feature in terms of good cycling stability, hence EIS was performed on brookite that was cycled previously in order to probe it further. The Nyquist Plots of pristine brookite and brookite cycled 10 times (Figure D.7) display a similar shape, but the cycled brookite has a much lower charge transfer resistance. This may be due to a small fraction of lithium that is irreversibly stored during the formation cycle (see Figure D.3, a feature common among the $\mathrm{TiO}_{2}$ polymorphs.[33] While this lowers the capacity slightly, it helps increase the conductivity of the crystal hence allowing for facile lithiation during cycling.

\subsubsection{XRD Probe of Litiated Brookite.}

The solid solution lithiation mechanism suggested by the slanted galavnostatic discharge curve (Figure 4.3 and 4.4a) was also confirmed structurally using XRD. Figure 4.5 shows XRD patterns for a pristine electrode; an electrode after the first lithiation; a delithiated electrode; and an electrode after 10 cycles. The pristine electrode shows peaks for both brookite and rutile, as were seen in the XRD of the powder (Figure 4.1), but also peaks for the PVDF binder. When lithiated, some of the brookite peaks no longer appear (112 and 231 peaks), while the rutile and PVDF peaks remain. This means that the rutile fraction $(27 \%)$ of the $\mathrm{TiO}_{2}$ nanoplatelet

\footnotetext{
${ }^{3}$ EIS performed over frequency range of 100,000 to $0.01 \mathrm{~Hz}$. Higher magnification of Figures 4.4c
} and $\mathrm{d}$ are located in Appendix D. 
material does not undergo lithiation in any measurable degree. With reference to brookite however, upon closer inspection (Figure D.8), the peak for brookite 121, seen in the pristine and delithiated materials, disappear and the peaks that do remain do not belong to brookite, but instead belong to orthorhombic $\mathrm{Li}_{0.5} \mathrm{TiO}_{2}$. $\mathrm{Li}_{0.5} \mathrm{TiO}_{2}$ does not have a peak at $2 \theta=30.8$, where the 121 peak is (hence its disappearance), as well, the main peak shifts from $2 \theta=25.69$ to $2 \theta=25.252$. This shift is subtle (indicated by dashed lines) due to peak broadening associated with lattice expansion from the insertion of lithium. In the previous work of Dambournet, et al.,[35] the studied lithiated brookite had the composition $\mathrm{Li}_{0.75} \mathrm{TiO}_{2}$, rather than $\mathrm{Li}_{0.5} \mathrm{TiO}_{2}$ as determined in this study. That composition however was attained only during the formation cycle followed by significant irreversible capacity loss in subsequent cycles, a loss not observed with the $\mathrm{Li}_{0.5} \mathrm{TiO}_{2}$ form of the present work (see Figure D.3) and as discussed in the next section. Hence $\mathrm{Li}_{0.5} \mathrm{TiO}_{2}$ better reflects the lithiated composition of brookite under reversible cycling conditions.

Upon delithiation (pattern III) the brookite peaks reform, and are sharper than the peaks of the lithiated $\mathrm{TiO}_{2}$. This is better seen with the XRD pattern obtained after cycling 10 times (Figure 4.5(IV)), where the characteristic brookite peaks are sharper and more intense than even the pristine material. This could signify the refinement of the crystal structure as the electrode cycles, a rather unique behavior that has gone unnoticed previously. This allows the rutile (211) peak to be seen in Figure 5(IV) which was not seen in the other patterns. Because of the refinement of the crystal, this peak is deconvoluted and visualized in the pattern. In one of the earlier studies[7] of brookite lithiation, "partial recovery of the crystallinity" was observed following the first lithiation cycle but not monitoring of the crystal structure evolution of brookite beyond the first cycle was done. It is proposed that the brookite crystal evolution behavior observed to occur during cycling, in combination with Dambournet et al's[35] modeling predictions that the brookite $\mathrm{TiO}_{2}$ framework retains its connectivity during lithiation, is a key property in rendering brookite very stable in terms of cycling as demonstrated with the data 
presented in Figure 4.6(bottom) and Figure D.3.

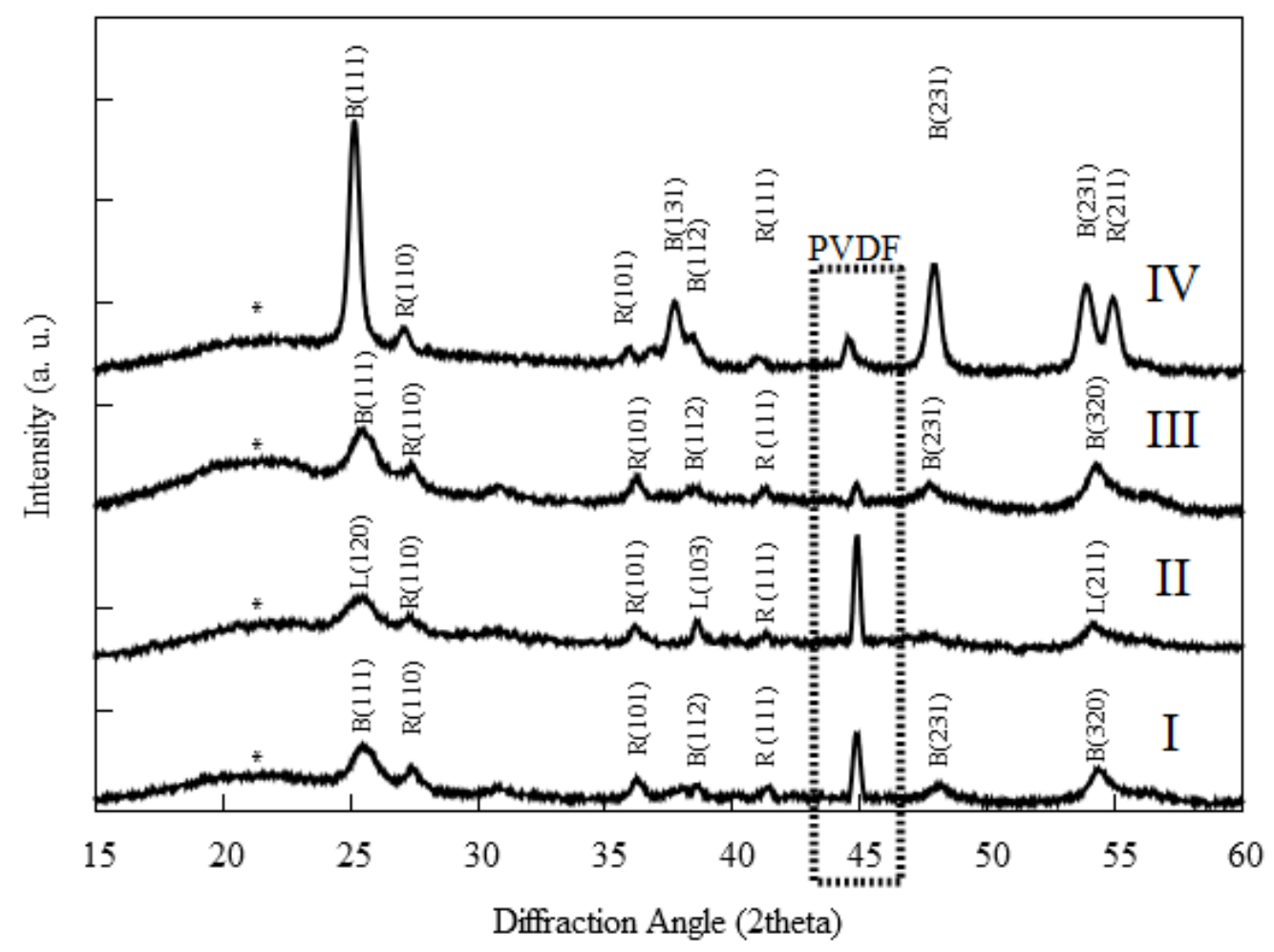

Figure 4.5: Diffraction patterns for pristine electrode (I), electrode after first lithation (II), electrode after first delithiation (III), and electrode after 10 cycles (IV). Amorphous humps caused by glass slide is marked with a "*" while the peaks for PVDF is indicated with an enclosure.

\subsubsection{Rate Capability and Cycling Stability.}

Figure 4.6a,b shows the rate capability and cyclability of the brookite material. At $\mathrm{C} / 10$ rate (where $\mathrm{C}=168 \mathrm{~mA} \mathrm{~g}^{-1}$ ) the discharge capacity of the brookite nanoplatelet active material (specific surface area $135 \mathrm{~m}^{2} \mathrm{~g}^{-1}$ ) was $183 \mathrm{~mA} \mathrm{~h} \mathrm{~g}^{-1}$. By comparison, the discharge capacities (2nd cycle) for other nanobrookite materi- 
als were: $200 \mathrm{mAhg}^{-1}$ (at $\mathrm{C} / 5$ ) for $10 \mathrm{~nm}$ nanocrystallites,[31] $185 \mathrm{~mA} \mathrm{hg}^{-1}$ (at $\mathrm{C} / 7.5$ ) for mesoporous brookite of high surface area $\left(250 \mathrm{~m}^{2} \mathrm{~g}^{-1}\right)$ [34] and $150 \mathrm{~mA} \mathrm{~h} \mathrm{~g}^{-1}(\mathrm{C} / 5)$ for $10-20 \mathrm{~nm}$ crystallites $\left(100 \mathrm{~m}^{2} \mathrm{~g}^{-1}\right)$.[32] As the rate increases, progressively the charge and discharge capacities decrease (Figure 4.6a) reflecting the relatively high Li-ion diffusion resistance $\left(Z_{W}\right.$ in Table D.1) and low electronic conductivity of $\mathrm{TiO}_{2}$. Thus at $1 \mathrm{C}$, the brookite naoplatelets register $135 \mathrm{~mA} \mathrm{~h} \mathrm{~g}^{-1}$ capacity that is reduced to $60 \mathrm{~mA} \mathrm{~h}^{-1}$ at $10 \mathrm{C}$. However, what is particularly impressive is the exceptional stability exhibited by the $\mathrm{mNPl}$ brookite material (mNP1 stands for mesoporous nanotlatelet) upon cycling. Figure $4.6 \mathrm{~b}$ shows the reversible capacity over multiple cycles at increasing rate. As it can be seen (consult also Figure D.3 and the full set of data given in Table D.2) mNP1 brookite's (cycled 50 times at $\mathrm{C} / 10$ rate) lithiation capacity is stabilized after the first formation cycle at $\sim 180 \mathrm{~mA} \mathrm{~h} \mathrm{~g}^{-1}$ and remains effectively constant. At the same time the coulombic efficiency is seen to approach $100 \%$ as the number of cycles increases, i.e. $>99.7 \%$ at C/10 (Table D.2). This is a rather unique characteristic of these mesoporous nanopleatelets among the different nano- $\mathrm{TiO}_{2}$ polymorphs not reported/observed previously, reflecting their discovered tendency to assume higher degree of crystallinity upon cycling as evident from the XRD data in Figure 4.5(IV). By contrast the nanobrookite materials previously studied have shown variable degrees of capacity fading. Thus the capacity of Reddy et al's nanocrystallites dropped from $200 \mathrm{~mA} \mathrm{~h}^{-1}$ to $170 \mathrm{~mA} \mathrm{~h} \mathrm{~g}^{-1}$ after 40 cycles at $\mathrm{C} / 5$; [31] the capacity of the mesoporous brookite of Dambournet et al dropped from 185 to $155 / 140 \mathrm{~mA} \mathrm{~h} \mathrm{~g}^{-1}$ (after 20/50 cycles at C/7.5)(32 and SI in ref 34); and the capacity of Lee et al's 10-20 nm crystallites dropped from 150 to only $50 \mathrm{~mA} \mathrm{~h} \mathrm{~g}^{-1}$ after 50 cycles (at 5C).[32] Finally, the mNP1 brookite material found to yield the same exceptional stability and coulombic efficiency $(99.8 \%$ ) cycled 10 times at high rate (10C) even after the electrode had been cycled 70 times previously at various rates. 

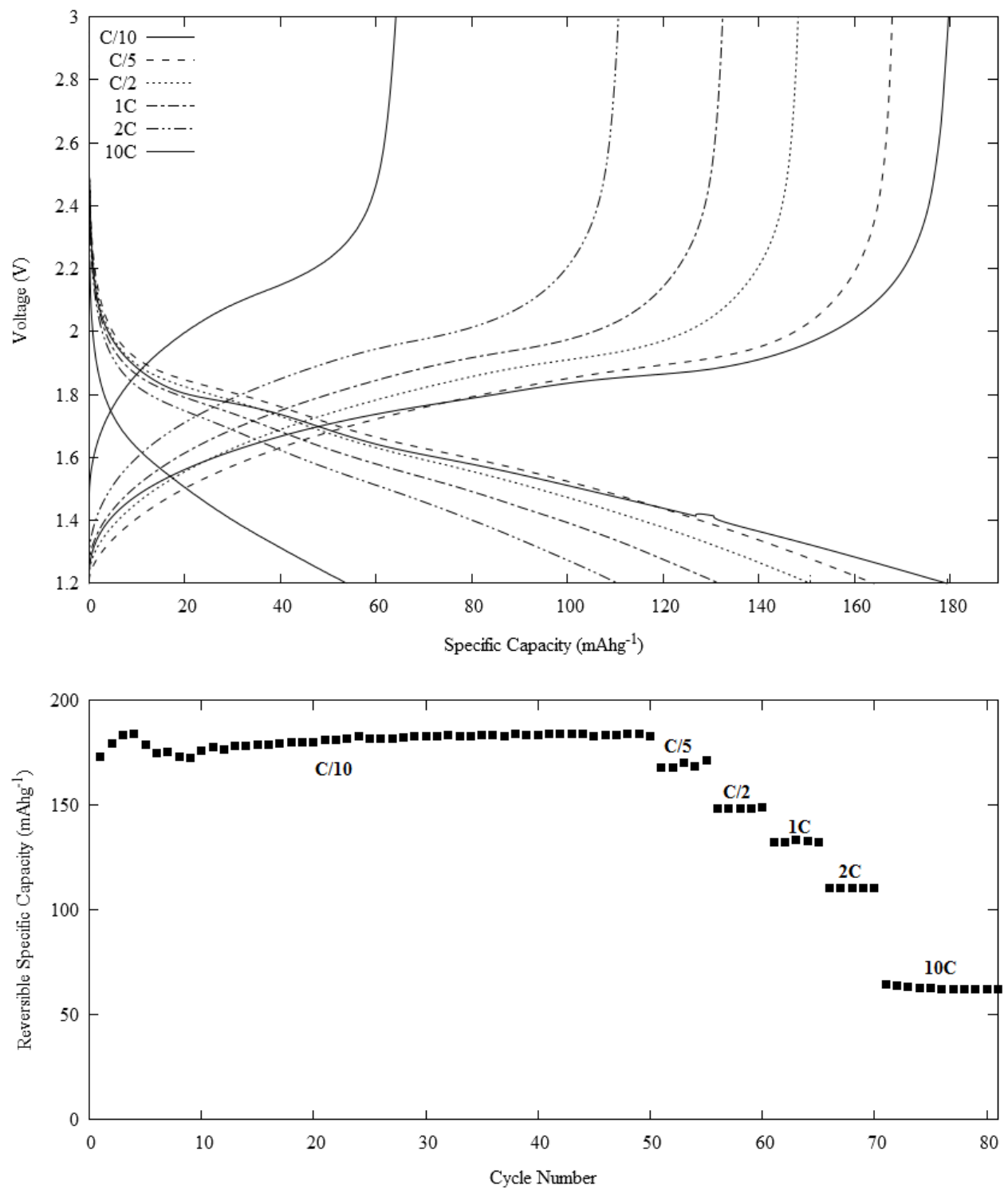

Figure 4.6: 2 nd cycle lithiation and delithiation profiles of the brookite nanoplatelets at various rates (top) and the lithiation (discharge) capacity retention of the brookite component over multiple cycles at various rates (bottom). The capacity values reflect the mass of active brookite only, not the total mass that comprises brookite and inactive rutile. 
The exceptional stability/high degree of reversible capacity retention at different rates reported in this work is attributed to the unique single crystal mesoporous brookite nanoplatelets that do not only provide short Li-ion diffusion path and excellent active material/carbon/electrolyte contacting, but also undergo crystal evolution during cycling into a highly flexible and relaxed structure. The mesoporous nanoplatelets allow for optimized material interaction with the electrolyte and short diffusion path for the intercalating/deintercalating Li-ions outperforming the most common nanoparticle-structured brookite varieties.[33, 49] The mNPls show better capacity at higher rate compared to other brookite varieties because of the short diffusion path. The increased diffusion resistance discussed previously is partially mitigated by the shortened path due to the unique morphology. This also has to do with the fact that a larger fraction of each nanoplatelet can be lithiated per unit time as compared to a spherical nanoparticle. Figure 4.7 shows that while both nanoparticles and nanoplatelets essentially undergo a core-shell diffusion front, the increased interaction with the electrolyte and favorable Li-ion diffusion perpendicular to the flat face of the nanoplatelets allows a greater fraction of the $\mathrm{TiO}_{2}$ to lithiate. In the meantime the progressive brookite crystal structure enhancement upon cycling despite the lattice distortion occurring during lithiation as discussed by Dambournet et al.[35] allows the nanoplatelets to quickly relax upon delithiation in an extremely efficient ( $>99.7 \%$ coulombic efficiency) reversible manner. Although at this stage, the origin of the discovered brookite crystallinity enhancement upon cycling, which contributes to excellent reversible stability, is not known it may very well be due to favorable nanoplatelet anisotropy as was discussed in the case of anatase by Belak et al.[17] Undoubtedly, further work focusing on understanding the crystal evolution mechanism and pursuing of strategies to improve rate capability via carbon coating[50] and/or doping can prove very valuable in fully exploiting the highly stable reversible cycling potential of the mesoporous brookite nanoplatelets. 


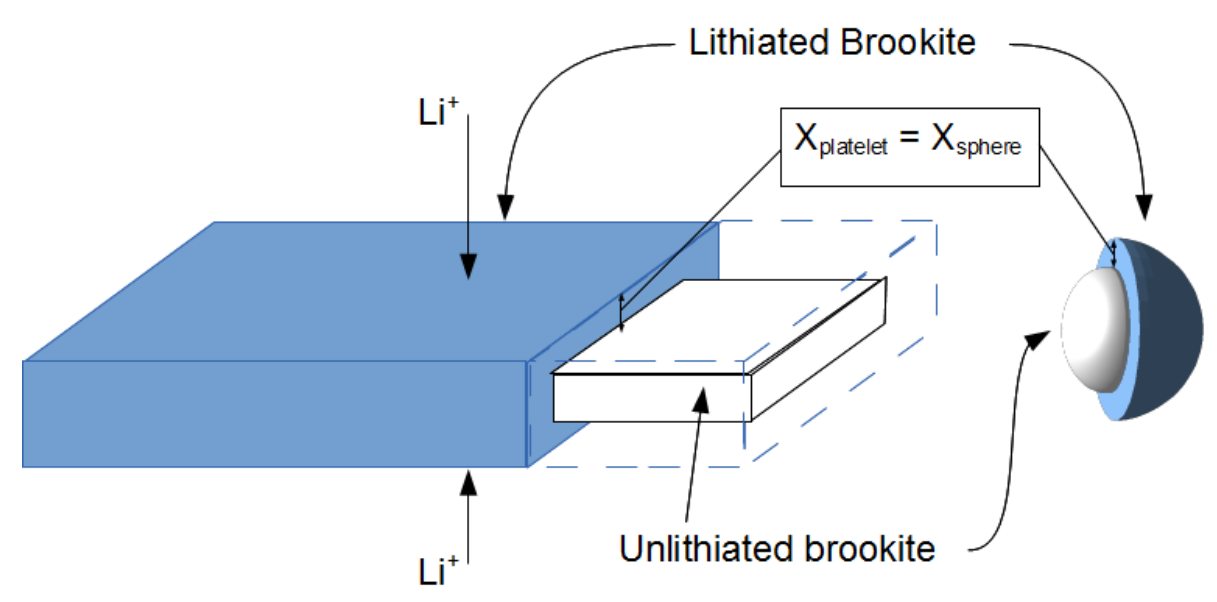

Figure 4.7: Schematic of nanoplatelet (left) and nanoparticle (right) after lithiation over a finite period of time.

\subsection{Conclusions}

A new variety of nano- $\mathrm{TiO}_{2}$ material comprising unique single crystal brookite mesoporous nanoplatelets has been synthesized via a novel aqueous solution process carried out in a continuous-stirred tank reactor. The synthesis involves forced hydrolysis of $\mathrm{TiCl}_{4}$ solution at $80^{\circ} \mathrm{C}$ over one hour reaction time. These unique mesoporous single crystal brookite nanoplatelets (mNPl; $135 \mathrm{~m}^{2} \mathrm{~g}^{-1}$ surface area with $\sim 5-10 \times 20 \times 50 \mathrm{~nm}$ dimensions and 2-8 nm pore size) provide favorable large electrolyte/active material interface, short Li-ion diffusion length, and stable structure, important considerations for good Li-ion intercalation performance. The brookite nanoplatelets achieved $183 \mathrm{~mA} \mathrm{~h} \mathrm{~g}^{-1}$ (2nd cycle) at $\mathrm{C} / 10$ rate (where $\mathrm{C}$ $=168 \mathrm{~mA} \mathrm{~g}^{-1}$ ), this being reduced to $130 \mathrm{~mA} \mathrm{~h} \mathrm{~g}^{-1}$ at $1 \mathrm{C}$ and $60 \mathrm{~mA} \mathrm{~h} \mathrm{~g}^{-1}$ at $10 \mathrm{C}$. In contrast to previously synthesized nanobrookite materials the $\mathrm{mNPl}$ brookite produced in this work was found to exhibit unparallel stability (reversible capacity retention) upon cycling. For example the reversible capacity of the mNP1 
brookite (cycled 50 times at $\mathrm{C} / 10$ rate) was stabilized after the first few cycles at $182 \mathrm{~mA} \mathrm{~h}^{-1}$. Coulombic efficiency also improved with cycling reaching a level $>99.7 \%$, independent of the applied rate. Post-mortem XRD characterization of lithiated and de-lithiated materials revealed the $\mathrm{mNPl}$ brookite's crystallinity to increase with cycling following lattice distortion during lithiation, a rather unique characteristic among the different nano- $\mathrm{TiO}_{2}$ polymorphs. According to galvanostatic, impedance and $\mathrm{x}$-ray diffraction and selected area electron diffraction analysis the lithiation of the brookite nanoplatelets was determined to proceed via solidsolution formation corresponding to orthorhombic $\mathrm{Li}_{0.5} \mathrm{TiO}_{2}$ that is isostructural with brookite. Overall it is concluded that the newly synthesized brookite material delivers exceptional stability/high degree of reversible capacity retention thanks to its unique single crystal mesoporous nanoplatelet structure that is highly flexible and relaxed following lithiation in addition to allowing short Li-ion diffusion path and excellent active material/carbon/electrolyte contacting.

\section{Acknowledgements}

The authors would like to thank Jeanne Clouteau for assisting with the fabrication of the electrodes, and Dr. Fuqiang Guo for his help with the synthesis tests. This research is supported with a strategic project grant from the Natural Sciences and Engineering Research Council of Canada (NSERC) and sponsored by Hydro-Quebec's Energy Conversion and Storage department.

\subsection{References}

[1] X. Su, Q.-L. Wu, X. Zhan, J. Wu, S. Wei, Z. Guo, J. Mater. Sci., 47, 2519 (2012).

[2] C. Jiang, J. Zhang, J. Mater. Schi. Technol., 29, 97 (2013). 
[3] M. Mancini, F. Nobili, R. Tossici, M. Wolfahrt-Mehrens, R. Marassi, J. Power Sources, 196(22), 9665 (2011).

[4] T. Hu, X. Sun, H. Sun, M. Yu, F. Lu, C. Liu, J. Lian, Carbon, 51, 322 (2013).

[5] J.-Y. Shin, D. Samuelis, J. Maier, Adv. Funct. Mater., 21(18), 3464 (2011).

[6] M. A. Reddy, M. S. Kishore, V. Pralong, V. Caignaert, U. V. Varadaraju, B. Raveau, Electrochem. Commun., 8(8), 1299 (2006).

[7] M. A. Reddy, M. S. Kishore, V. Pralong, U. V. Varadaraju, B. Raveau, Electrochem. Solid St., 10(2), A29 (2007).

[8] B. Zhao, R. Cai, S. Jiang, Y. Sha, Z Shao, Electrochim. Acta, 85, 636 (2012).

[9] S. Brutti, V. Gentili, H. Menard, B. Scrosati, P. G. Bruce, Adv. Energy Mater, 2(3), 322 (2014).

[10] R. Yu, Z. Liu, F. Pourpoint, A. R. Armstrong, C. P. Grey, P. G. Bruce, Angew. Chem. Int. Ed., 51, 2164 (2012).

[11] S. Liu, Z. Wang, C. Yu, H. B. Wu, G. Wang, Q. Dong, J. Qiu, A. Eychmuller, X. W. Lou Adv. Mater, 25, 3462 (2013).

[12] W. J. Borghols, D. Lutzenkirchen-Hecht, U. Haake, E.R. van Eck, F. M. Mulder, M. Wagemaker, Phys. Chem. Chem. Phys., 11, 5742 (2009).

[13] M. Wagemaker, W. J. Borghols, F. M. Mulder, J. Am. Chem. Soc., 129(14), 4323 (2007).

[14] S. K. Panda, Y. Yoon, H. S. Jung, W.-S. Yoon, H. Shin, J. Power Sources, 204, 162 (2012).

[15] H. Lindstrom, S. Sodergren, A. Solbrand, H. Rensmo, J. Hjelm, A. Hagfeldt, S.-E. Lindquist, J. Phys. Chem. B, 101(39), 7717 (1997).

[16] C. Jiang, M. Wei, Z. Qi, T. Kudo, I. Honma, H. Zhou, J. Power Sources, 166, 239 (2007).

[17] A. A. Belak, Y. Wang, A. Van der Ven, Chem. Mater, 24(15), 2894 (2012). 
[18] W. J. Borghols, M. Wagemaker, U. Lafont, E. M. Kelder, F. M. Mulder, Chem. Mater., 20, 2949 (2008).

[19] Y. Ma, G. Ji, B. Ding, J. Y. Lee, J. Mater. Chem., 22, 24380 (2012).

[20] D. Bresser, E. Paillard, E. Binetti, S. Krueger, M. Striccoli, M. Winter, S. Passerini, J. Power Sources, 206, 301 (2012).

[21] J. S. Chen, L. A. Archer, X. W. Lou, J. Mater. Chem., 21(27), 9912 (2011).

[22] A. G. Dylla, P. Xiao, G. Henkelman, K. J. Stevenson, J. Phys. Chem. Lett., 3, 2015 (2012).

[23] M. Fehse, F. Fisher, C. Tessier, L. Stievano, L. Monconduit, J. Power Sources, 231, 23 (2013).

[24] M. Koudriachova, N. Harrison, S. de Leeuw, Phys. Rev. B, 65(23), 1 (2002).

[25] P. Kubiak, M. Pfanzelt, J. Geserick, U. Hormann, N. Husing, U. Kaiser, M. Wohlfarht-Mehrens, J. Power Sources, 194, 1099 (2009).

[26] Y. S. Hu, L. Kienle, Y.-G. Guo, J. Maier, Adv. Mater., 18, 1421 (2006).

[27] J. S. Chen and X. W. Lou, J. Power Sources, 195, 2905 (2010).

[28] Z. Hong, W. Wei, T. Lan, G. Cao, Nano Energy, 1, 466 (2012).

[29] D. W. Murphy, R. J. Cava, S. M. Zaburak, A. Santoro, Solid State Ionics, 9 \& 10, $413(1983)$.

[30] A. Beltran, L. Garcia, J. Andres, J. Phys. Chem. B, 110, 23417 (2006).

[31] M. A. Reddy, V. Pralong, U. V. Varadaraju, B. Raveau, Electrochem. Solid St., 11(8), A132 (2008).

[32] D.-H. Lee, J.-G. Park, K. J. Choi, H.-J. Choi, D.-W. Kim, Eur. J. Inorg. Chem., 2008(6), 878 (2008).

[33] D. Dambournet, I. Belharouak, K. Amine, Chem. Mater, 22, 1173, (2010). 
[34] D. Dambournet, I. Belharouak, J. Ma, K. Amine, J. Mater. Chem., 21, 3085 (2011).

[35] D. Dambournet, K. W. Chapman, M. V. Koudriachova, P. J. Chupas, I. Belharouak, K. Amine, Inorg. Chem., 50(13), 5855 (2011).

[36] C. Charbonneau, R. Gauvin, G. P. Demopoulos, J. Electrochem. Soc, 158(3), H224 (2011).

[37] A. Yasin, F. Guo, G. P. Demopoulos, Materials Research Society Spring Meeting, (2013).

[38] J. Liu and X.-W. Liu, Adv. Mater., 24, 4097 (2012).

[39] J. S. Chen and X. W. Lou, Electrochem. Commun., 11(12), 2332 (2009).

[40] H. Zhang. and J. F. Banfield, J. Phys. Chem. B, 104, 3481 (2000).

[41] J. A. Dirksen and T. A. Ring, Chem. Eng. Sci., 46, 2389 (1991).

[42] G. P. Demopoulos, Hydrometallurgy, 96, 199 (2009).

[43] J. B. Condon, Surface Area and Porosity Determination by Physisorption, Elsevier Science (2006).

[44] C. Ducati, Nature, 495, 180 (2013).

[45] E. J. W. Crossland, N. Noel, V. Sivaram, T. Leijtens, J. A. Alexander-Webber and H. J. Snaith, Nature, 495, 215 (2013).

[46] B. Ohtani, O. O. Prieto-Mahaney, D. Li, R. Abe, J. Photoc.Photobio., 216, 179 (2010).

[47] M. J. Sussman, N. Brodusch, R. Gauvin, G. P. Demopoulos, J. Electrochem. Soc., 160(5), A3100 (2013).

[48] N. Ogihara, S. Kawauchi, C. Okuda, Y. Itou, Y. Takeuchi and Y. Ukyo, J. Electrochem. Soc., 159, A1034 (2012).

[49] D.-H. Lee, J.-G. Park, D.-W. Kim, Cryst. Growth Des., 8, 4506 (2008).

[50] J. Wang and X. Sun, Energy. Environ. Sci., 5, 5163 (2012). 



\section{Chapter 5}

\section{On the Complex Interplay of Crystallinity and Surface Area Effects on Li-Ion Intercalation and Pseudocapacitive Storage Properties of Nanocrystalline Anatase}

As the phase of choice, anatase is the most widely studied of the $\mathrm{TiO}_{2}$ phases. Though many of these studies have developed synthesis methods for various morphologies, they tend toward complex chemistry, harsh chemicals, and low output. However, by manipulating the aqueous synthesis technique explained in the previous chapter, we are able to easily produce anatase with a small particle size and high surface area. As such, it makes an excellent candidate as a high rate electrode material. Further, because the as-synthesized particles are not very crystalline, we have the opportunity to test claims that highly crystalline materials have better properties at high rate. The aqueous anatase is therefore characterized physically and electrochemically to determine both its properties and the role surface area and crystallinity play in lithium storage at high rate (previously published in: M. J. Sussman, A. Yasin, G. P. Demopoulos, On the Complex Interplay of Crystallinity and Surface Area Effects on Li-Ion Intercalation and Pseudocapacitive Storage Properties of Nanocrystalline Anatase, Journal of Power Sources, 272, (2014) 58-67). 


\subsection{Abstract}

Lithium insertion in anatase is studied by considering simultaneously the effects of crystallinity and surface area on storage mechanism, namely intercalation vs. pseudocapacitive. $6 \mathrm{~nm}$ anatase crystallites with high surface area $\left(222 \mathrm{~m}^{2} \mathrm{~g}^{-1}\right)$, synthesized via a novel continuous aqueous process and annealed at different temperatures $\left(200^{\circ} \mathrm{C}, 300^{\circ} \mathrm{C}\right.$, and $\left.400^{\circ} \mathrm{C}\right)$, were electrochemically tested. By annealing, crystallinity was increased, while surface area decreased allowing for the investigation of the contributions of each toward lithiation and delithiation behavior. The as-synthesized and best-annealed (at $300^{\circ} \mathrm{C}$ ) samples were compared at various rates. At low rate ( $1 \mathrm{C}$ and $2 \mathrm{C}$ ) the annealed anatase had higher reversible capacity than the as-synthesized, due to increased diffusion-based storage. At high rate however, as-synthesized anatase had much higher capacity due to increased surface area and ability to store Li-ions pseudocapacitively. Increased crystallinity leads to increased diffusivity as determined by electrochemical impedance spectroscopy, explaining why the $300^{\circ} \mathrm{C}$ anneal had higher capacity at low rate. Long-term cycling at high rate, however, showed that reliance on diffusion-based storage (intercalation) in the case of the $300^{\circ} \mathrm{C}$ anneal causes increased polarization that leads to electrode performance decline. These results point to the importance of simultaneous nanoanatase property (crystallinity and surface area) optimization for achieving stable performance.

\subsection{Introduction}

Various nanophases of $\mathrm{TiO}_{2}$ have been evaluated as active material for anodes in lithium ion batteries [1--3]. Research focusing on each of the naturally occurring phases (rutile, anatase, and brookite) [4--8], as well as synthetic $\mathrm{TiO}_{2}(\mathrm{~B})[9,10]$, have all shown that $\mathrm{TiO}_{2}$ has promise as a replacement for the current commercially used graphitic materials. Part of this is ascribed to the pseudocapacitive storage 
that is seen in $\mathrm{TiO}_{2}$, not associated with graphite [11--15].

Pseudocapacitance presents itself in $\mathrm{TiO}_{2}$ as reversible surface storage [16]. However, this is only a partial contribution to the capacity. This contribution is usually ascribed to the amount of surface area associated with active material particles $[11,17]$. The other contributing storage mechanism is diffusion-based lithium insertion and extraction that is accompanied by crystallographic phase change as per the following reaction:

$$
\mathrm{TiO}_{2}+x\left(\mathrm{Li}^{+}+\mathrm{e}^{-}\right) \Longleftrightarrow \mathrm{Li}_{\mathrm{x}} \mathrm{TiO}_{2}
$$

Insertion of lithium into $\mathrm{TiO}_{2}$, and anatase in particular, can theoretically reach $\mathrm{x}=$ 1 in $\mathrm{Li}_{\mathrm{x}} \mathrm{TiO}_{2}$, which translates to $335 \mathrm{~mA} \mathrm{~h} \mathrm{~g}^{-1}$, however, practical electrochemical lithiation occurs to $\mathrm{x}=0.5\left(168 \mathrm{~mA} \mathrm{~h}^{-1}\right)$ [18]. Part of this has to do with the fact that during diffusive insertion anatase undergoes a phase change from tetragonal $\mathrm{TiO}_{2}$ to orthorhombic $\mathrm{Li}_{0.5} \mathrm{TiO}_{2}$ and then to tetragonal $\mathrm{LiTiO}_{2}[19,20]$. Belak, et al. [20] explained this transition as a full particle transition, determining that a particle can either be $\mathrm{TiO}_{2}$ or $\mathrm{Li}_{0.5} \mathrm{TiO}_{2}$ or $\mathrm{LiTiO}_{2}$ but not any two at one time, whereas Borghols, et al. [19] stated that a layer of $\mathrm{LiTiO}_{2}$ diffuses $4 \mathrm{~nm}$ deep within the $\mathrm{TiO}_{2}$ particle making the $\mathrm{x}$ in $\mathrm{Li}_{\mathrm{x}} \mathrm{TiO}_{2}$ a function of particle depth, thus by shrinking the particle size, from bulk to nano $(\sim 7 \mathrm{~nm})$, the fraction of the particle that was converted to $\mathrm{LiTiO}_{2}$ would increase to $100 \%$. This, however, was in direct disagreement with Jiang, et al. [21] who demonstrated that for $7 \mathrm{~nm}$ particles (as compared to $30 \mathrm{~nm}$ particles) lithiation by diffusion was only part of the process.

The $7 \mathrm{~nm}$ particles (commercially synthesized) showed shorter diffusion plateaus than their $30 \mathrm{~nm}$ counterparts, which indicated that surface-based storage played a role as important in the storage of charge as diffusion-based storage[21]. As well, Kang, et al. [22] showed that the optimal particle size range was 8-25 nm, determined by annealing $5 \mathrm{~nm}$ particles synthesized via solvothermal methods. The $8 \mathrm{~nm}$ particle material had the best capacity even at high rate (20C), namely $75 \mathrm{~mA} \mathrm{~h} \mathrm{~g}^{-1}$, 
by contrast their as-synthesized crystallites $(5 \mathrm{~nm})$ performed extremely poorly, something that was ascribed to poor crystallinity. However, they did not investigate further these differences to identify the underlying causes.

Further, Belak, et al. [20] also explained that, from a diffusion stand point, by changing the aspect ratio, rather than just shrinking the particle, facile insertion of lithium could occur leading to higher capacity even at high rate. Synthesis of non-spherical particles has provided increasing lithiation capacity. Chen and Lou [23] produced nanosheets via a hydrothermal method using titanate isopropoxide, that achieved 120 and $112 \mathrm{~mA} \mathrm{~h} \mathrm{~g}^{-1}$ at $10 \mathrm{C}$ and $20 \mathrm{C}$, respectively, however their anatase saw large capacity fading at high rate. In the meantime Shim, et al. [24], who synthesized high surface area $\left(95 \mathrm{~m}^{2} \mathrm{~g}^{-1}\right)$ nanowires, achieved $200 \mathrm{~mA} \mathrm{~h} \mathrm{~g}^{-1}$ at $1 \mathrm{C}$ and $115 \mathrm{~mA} \mathrm{~h}^{-1}$ at $20 \mathrm{C}$. The short diffusion distance allowed for facile lithiation at high rate allowing more capacity even with a surface area lower than that of the nanoparticles characterized by Jiang, et al. Finally Bresser, et al. [25] had impressive intercalation results with nanorods $3-4 \mathrm{~nm}$ in diameter, achieving $250 \mathrm{~mA} \mathrm{~h} \mathrm{~g}^{-1}$ at $\mathrm{C} / 10$ and $130 \mathrm{~mA} \mathrm{~h} \mathrm{~g}^{-1}$ at $10 \mathrm{C}$ rate.

While all of this work has focused on the intercalation properties of anatase at both high and low rate, the contributions from faradaic (bulk insertion) and non-faradaic (surface-area controlled capacitance) have not been clearly distinguished. The ability of nanoparticles to deliver good Li-ion storage performance over longer cycling ranges at high rate is usually attributed to shortened diffusion length $[26,27]$. Wang, et al. [26] synthesized porous nanoparticles that showed excellent cycling stability at high rate $\left(\sim 100 \mathrm{~mA} \mathrm{~h}^{-1}\right.$ after 100 cycles at $\left.20 \mathrm{C}\right)$, and though their particles had a high specific surface area, they ascribed the high capacity and retention to shortened diffusion paths. The possibility of pseudocapacitive storage associated with the high rate was never mentioned.

Presently, to the best of our knowledge, there is no published work focusing on the effects of both crystallinity and surface area on high rate Li-ion storage in nanoscale anatase. While Kang, et al. [22] mentioned the low crystallinity of 
their particles as a reason for poor performance, they did not quantify this, nor did they compare to the crystallinity of their annealed samples. The other previously mentioned works assumed that their nano-anatase was highly crystalline, leaving questions as to the effect that crystallinity has had on the performance of their material. Further, as the anatase investigated is usually high surface area and thus a small crystallite size, it seems that it is simply assumed that the diffusion length is the primary reason for increased capacity without any mention of the surface area-based storage.

Recent research in our laboratory focusing on the forced hydrolysis of aqueous $\mathrm{TiCl}_{4}$ solution [28], has led to the discovery that $\sim 6 \mathrm{~nm}$ anatase crystallites can be produced and recovered via fast (30 min residence time), low temperature (at $80^{\circ} \mathrm{C}$ ) reaction carried in a continuous-stirred reactor at $\mathrm{pH} 3$ [29]. The nanoparticles produced possess high surface area, but are poorly crystalline giving an opportunity to understand the role crystallinity and surface area play in lithium intercalation and pseudocapacitance upon annealing. It is thus the goal of this work to report on the electrochemical energy storage properties of a new type of anatase nanoparticles synthesized via a high throughput continuous aqueous solution process and to elucidate the effect that both crystallinity and surface area play on the mechanism of Li-ion storage and cyclability at low and high rate.

\subsection{Experimental}

\subsubsection{Synthesis and Characterization}

Anatase nanoparticles were synthesized using a continuous aqueous process developed in our laboratory [29], based on a modification of an earlier batch method developed by Charbonneau, et al. [28] In a typical synthesis run, a $0.1 \mathrm{M} \mathrm{TiCl}_{4(\mathrm{aq})}$ solution was continuously pumped into a stirred reactor preheated to $80{ }^{\circ} \mathrm{C}$ and neutralized with $\mathrm{NH}_{4} \mathrm{OH}$ or $\mathrm{NaOH}$ to $\mathrm{pH}=3$. After the attainment of steady-state 
operation at 30 minute mean residence time, a colloidal nanoanatase suspension was produced and separated by centrifugation. The material was washed 3 times in deionized water and once with anhydrous ethanol, then dried at $80^{\circ} \mathrm{C}$ overnight. The resulting solid was mechanically ground prior to its use and characterization.

As-prepared anatase samples were annealed at $200^{\circ} \mathrm{C}, 300^{\circ} \mathrm{C}$, and $400{ }^{\circ} \mathrm{C}$ for 24 hours to increase crystallinity. The samples were characterized by X-ray Diffraction (XRD), performed with a Bruker D8 Discover Diffractometer equipped with a copper target $\left(\lambda_{C u K \alpha 1}=0.15406 \mathrm{~nm}\right)$, to identify the crystal phases present. The degree of crystallinity was determined by comparing the integrated area under the broad (amorphous) hump and sharp crystalline peak corresponding to the (101) anatase crystal plane. Further, electrodes cycled 500 times at high rate were examined using XRD. The electrodes were removed from the cells, washed in dimethyl carbonate (DMC) and dried under argon. They were then mounted on glass microscope slides with carbon tape. Brunauer-Emmett-Teller (BET) surface area was used to determine particle size and surface area, using a Micromeritics TriStar 3000 apparatus. All samples weighed approximately $0.1 \mathrm{~g}$ and were degassed for 24 hours at $80^{\circ} \mathrm{C}$ prior to nitrogen physisorption.

A Philips CM200 Transmission Electron Microscope (TEM) was used at $200 \mathrm{kV}$ to determine particle morphology and crystal structure. TEM specimens were prepared by depositing particles on a copper grid. Selected-Area Electron Diffraction (SAED) for crystal structure analysis was conducted using a double-tilt specimen holder ( 2 different rotation axes with a maximum tilt angle of $\sim 20^{\circ}$ ).

\subsubsection{Electrochemical Measurements}

All electrochemical testing was performed via a BioLogic VSP Potentiostat/Galvanostat/EIS system using Swagelok-type half-cells assembled in an argon-filled glovebox. The as-prepared and annealed anatase samples were used as active materials for the working electrodes, while lithium foil was used as the counter electrode. 
Electrode sheets were fabricated using $80 \%$ active material, $10 \%$ acetylene black, and 10\% poly(vinylidene) fluoride (PVDF, HSV 900, Solvay) binder dissolved in 1-methyl 2-pyrrolidone (NMP, Sigma Aldrich) solvent. The resulting slurry was then doctor bladed onto aluminum foil sheets and dried at $120^{\circ} \mathrm{C}$ for 24 hours. The electrolyte used in this work was a solution of $1 \mathrm{M} \mathrm{LiPF}_{6}$ in EC:DMC:DEC (2:2:1 by weight) mixed solution (BASF).

Galvanostatic charge/discharge testing was performed on the cells with cutoff voltages at 3 and $1 \mathrm{~V}$. A specific current of $168 \mathrm{~mA} \mathrm{~g}^{-1}$ (corresponding to $1 \mathrm{C}$ rate) was used for both charging and discharging. Cells were first discharged to insert Li-ions into pristine $\mathrm{TiO}_{2}$, and then charged. Testing at $1 \mathrm{C}$ rate was carried out over 100 cycles for as-prepared anatase only. For all samples, cycling at $10 \mathrm{C}$ rate was performed for 100 cycles (and again for 500 cycles for as-prepared and $300{ }^{\circ} \mathrm{C}$ annealed anatase). Rate capability was performed on all samples at specific currents representing 1C, 2C, 5C, 10C, 20C, and back to $1 \mathrm{C}$. Each sample was cycled 10 times at $1 \mathrm{C}$ then 5 times at the subsequent rates.

Electrochemical Impedance Spectroscopy (EIS) was performed on each sample to understand the electrode behavior and diffusion at different states of charge (SOC). The technique used alternating current (AC) impedance, in which AC modulation of known amplitude $(5 \mathrm{mV})$ across a frequency range $(0.5 \mathrm{mHz}$ to 1 $\mathrm{MHz}$ ) is used to measure the in phase and out of phase components. The diffusion coefficient for lithium ion transport was calculated using the Warburg impedance as determined from EIS measurements.[30]

\subsection{Results and Discussion}

\subsubsection{Nanoanatase Characterization}

Figure 5.1 shows the $\mathrm{X}$-ray diffraction $(\mathrm{XRD})$ patterns of the synthesized anatase, both as-prepared and after annealing at $200^{\circ} \mathrm{C}, 300^{\circ} \mathrm{C}$, and $400^{\circ} \mathrm{C}$ (referred to 
as A0, A200, A300, and A400, respectively). The XRD patterns for all of the samples show a main peak at $2 \theta \approx 25.3^{\circ}$, which is in good agreement with the peak corresponding to the (101) plane of anatase. The other peaks seen in the diffraction patterns correspond to anatase, thus there is no evidence of impurity peaks suggesting the formation of a single phase. Percent crystallinity was determined mathematically by comparing the amorphous area with the crystalline area under the pattern (individual patterns are available in Figure S1 and the method is described in detail in the Supporting Information section of this paper). Table 5.1 shows the key physical characterization data for the different anatase nanomaterials. A0 is the least crystalline (70.1\%), while A400 is the most (90.5\%). Using Scherrer's equation, the crystallite size was determined for each sample. As with the percent crystallinity, the crystallite size increases as the anneal temperature increases.

In addition to Scherrer's equation used for nanocrystallite size determination that is known to be associated with error [31], the samples were characterized using Brunauer-Emmett-Teller (BET) surface area and transmission electron microscopy (TEM) techniques. The BET results (Table 1) show that as the anatase is annealed it undergoes a decrease in surface area from $222.6 \mathrm{~m}^{2} \mathrm{~g}^{-1}$ (A0) to $99.6 \mathrm{~m}^{2} \mathrm{~g}^{-1}$, as expected, which corresponds to an increase in crystallite size from 6.37 to 14.24

Table 5.1: Key physical characterization values for the different anatase nanomaterials.

\begin{tabular}{lllll}
\hline $\begin{array}{l}\text { Sample } \\
\text { Name }\end{array}$ & $\begin{array}{l}\text { Percent } \\
\text { Crystallinity }\end{array}$ & $\begin{array}{l}\text { Crystallite } \\
\text { Size* }[\mathrm{nm}]\end{array}$ & $\begin{array}{l}\text { Surface } \\
\text { Area } \\
{\left[\mathrm{m}^{2} \mathrm{~g}^{-1}\right]}\end{array}$ & $\begin{array}{l}\text { BET } \\
\text { Crystallite } \\
\text { Size }[\mathrm{nm}]\end{array}$ \\
\hline A0 & $70.1 \%$ & 9.578 & 222.6 & 6.37 \\
A200 & $74.3 \%$ & 10.322 & 158.7 & 8.93 \\
A300 & $89.5 \%$ & 12.513 & 120.1 & 11.8 \\
A400 & $90.5 \%$ & 15.062 & 99.6 & 14.24 \\
\hline \multicolumn{5}{c}{ *Based on Scherrer's method }
\end{tabular}




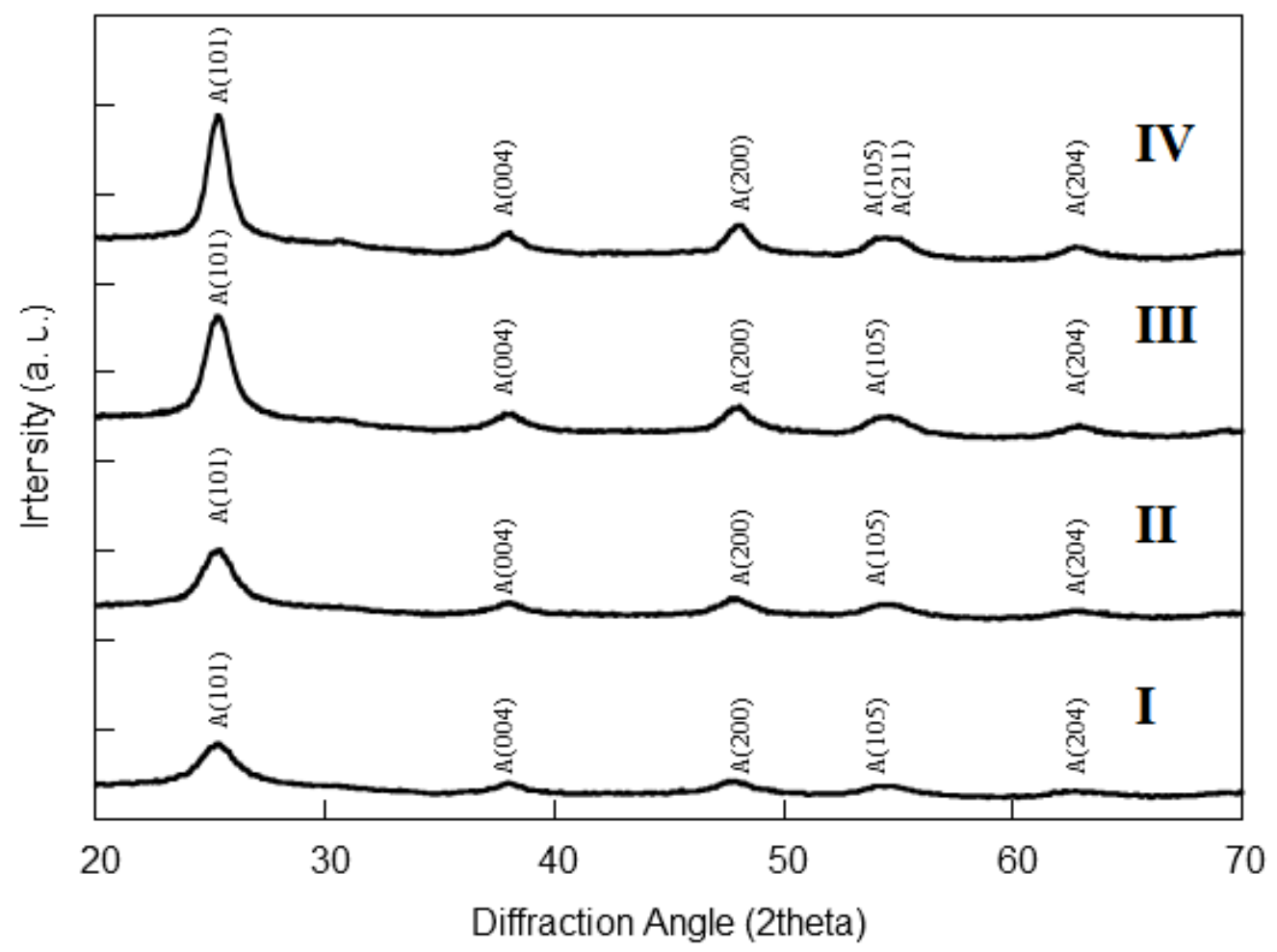

Figure 5.1: $\mathrm{X}$-ray diffraction patterns for as-prepared anatase (A0; I), and anatase samples annealed at $200^{\circ} \mathrm{C}(\mathrm{A} 200 ; \mathrm{II}), 300^{\circ} \mathrm{C}\left(\mathrm{A} 300\right.$; III), and $400^{\circ} \mathrm{C}$ (A400, IV) 
$\mathrm{nm}$. The shape of the isotherm associated with the anatase samples is indicative of a non-porous nanosolid, implying that the crystallite size calculated from the BET surface area measurements should better represent the true size and not the Scherrer method calculated size. This is corroborated with the TEM micrographs, seen in Figure 5.2, which show that the anatase crystallites are non-porous spherical nanoparticles. Particles in the micrograph of A0 measure approximately $6 \mathrm{~nm}$ in diameter, while A200, A300, and A400, measure approximately 9, 12, and $15 \mathrm{~nm}$ in diameter respectively. SAED was also performed on each sample to verify the phase of the particles as anatase (Figure S2).

The decrease in surface area seen in the annealed samples should have an effect on the non-faradaic storage associated with $\mathrm{TiO}_{2}[21,32]$. By lowering the surface area, there will be less surface sites for Li-ions to store. However, by increasing the crystallinity, diffusion should proceed more rapidly as the structure is more ordered, thus increasing the capacity seen at high rate. Annealing has been suggested to lead to high-rate capacity and cycling performance in $\mathrm{TiO}_{2}$ as well as other materials $[26,33,34] . \mathrm{In} \mathrm{TiO}_{2}$ the increase in capacity due to high crystallinity is postulated by Wang, et al. to be due to a more ordered phase transition from tetragonal $\mathrm{TiO}_{2}$ to orthorhombic $\mathrm{Li}_{0.5} \mathrm{TiO}_{2}$ [26]. However, this may have also come from the fact that they used $10 \mathrm{~nm}$ mesoporous nanoparticles, allowing for an increase in surface sites available for lithium storage rather than increased diffusion-based storage. This is deduced from the voltage profiles associated with the lithiation of their particles at various rates [21]. As the rate increased, the diffusive plateau was reduced until, at high rate, the profile consisted of an initial voltage drop then a sloping line that could be attributed to pseudocapacitive behavior.[14] 


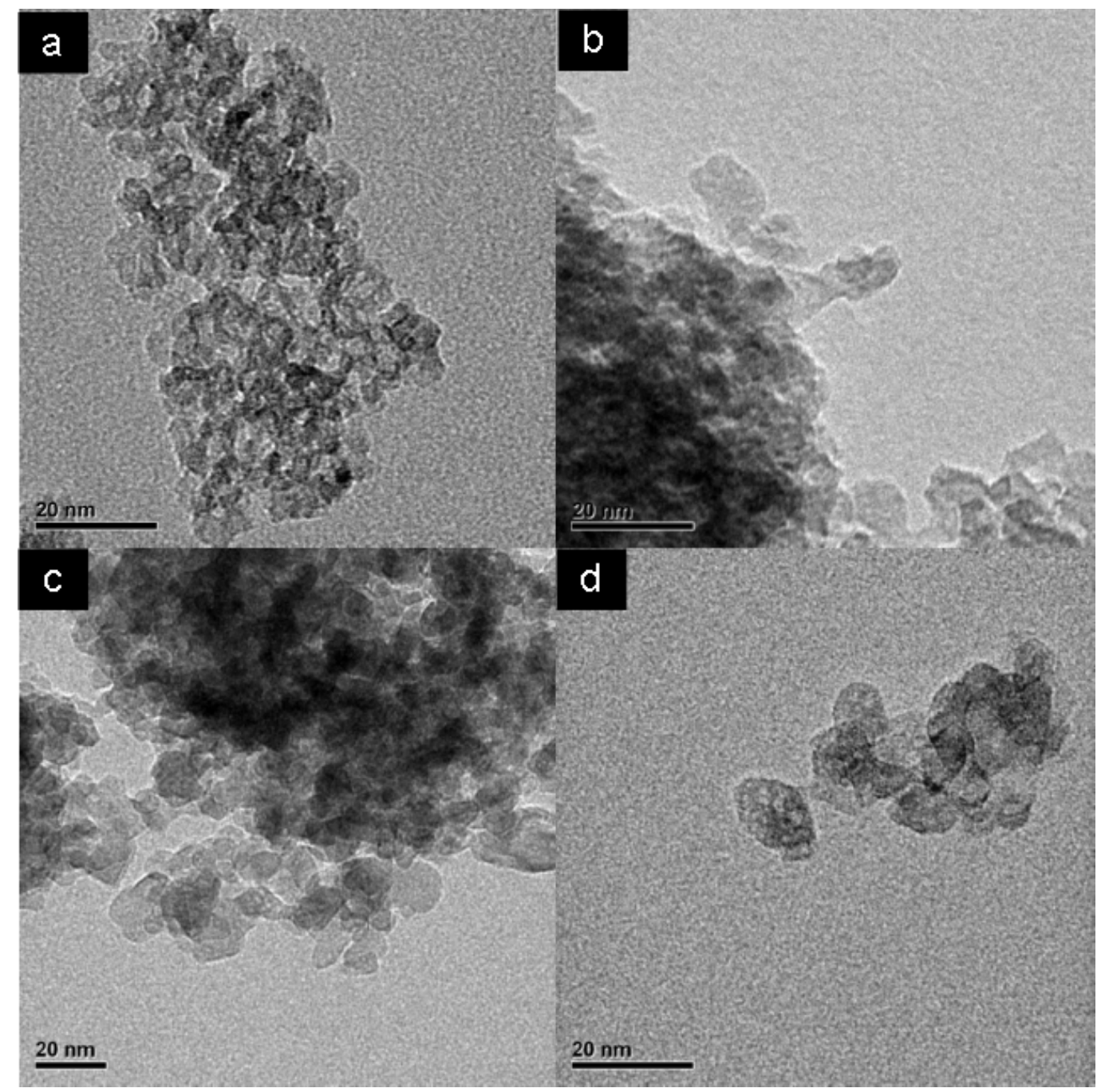

Figure 5.2: Transmission electron micrographs of A0 (a), A200 (b), A300 (c), and A400 (d) anatase samples. 


\subsubsection{Investigation of Storage Mechanisms}

\subsubsection{Galvanostatic Charge/Discharge.}

The charge/discharge performance of A0 was evaluated to understand the lithiation behavior of the as-prepared nanoanatase. Galvanostatic cycling was carried out (Figure S3) between $3 \mathrm{~V}$ (charge limit) and $1 \mathrm{~V}$ (discharge limit). During the formation cycle, A0 achieved a specific capacity of $230 \mathrm{~mA} \mathrm{~h} \mathrm{~g}^{-1}$ after lithiation, but the delithiation capacity was $195 \mathrm{~mA} \mathrm{~h}^{-1}$. This is in good agreement with the work of Jiang, et al., who achieved similar lithiation and delithiation capacities for $6 \mathrm{~nm}$ anatase nanoparticles [21]. However, after the initial lithiation and delithiation, there is capacity fading before the capacity stabilizes. The coulombic efficiency is almost $100 \%$ over 100 cycles leading to a specific reversible capacity of $\sim 150 \mathrm{~mA} \mathrm{~h} \mathrm{~g}^{-1}$. In other works focusing on $6 \mathrm{~nm}$ anatase nanomaterial, a higher reversible capacity is reported, but these values were obtained at lower rate or with particles having a unique morphology [35--37]. Sudant, et al. obtained a $165 \mathrm{~mA} \mathrm{~h} \mathrm{~g}^{-1}$ reversible capacity for $6 \mathrm{~nm}$ nanoparticles with $223 \mathrm{~m}^{2} \mathrm{~g}^{-1}$ surface area, however, this was obtained at $\mathrm{C} / 20$ rate, and after 50 cycles was only at $90 \%$ capacity retention [35]. Zhang, et al. [37] achieved $172 \mathrm{~mA} \mathrm{~h}^{-1}$ but were using mesoporous hollow spheres as their active material. In all these studies the authors invoked an intercalation type of storage arguing the benefits of nanostructuring in terms of a short diffusion length for lithium within the particle.

However, as already mentioned earlier pseudocapacitive storage may also play a role in this system especially at high rate (10C), a central issue of research focus in the present work. In order to fully understand the relationship between crystallinity and surface area of nanoanatase on type of Li-ion storage, all of the samples were cycled at $10 \mathrm{C}$ rate for 100 cycles (Figure 5.3). The Figure shows that A0 achieves the highest capacity after 100 cycles at $115 \mathrm{~mA} \mathrm{~h} \mathrm{~g}^{-1}$. While the trend in capacity mirrors that of A0, A200 has a much lower initial capacity and after 100 cycles,

its final capacity is $90 \mathrm{~mA} \mathrm{~h}^{-1}$. A300 has a different trend in capacity than A0 
and A200. After the initial cycle, the capacity retention is higher. The initial cycle has a lower reversible capacity than $\mathrm{A} 0\left(121 \mathrm{~mA} \mathrm{~h} \mathrm{~g}^{-1}\right.$ compared to $\left.134 \mathrm{~mA} \mathrm{~h} \mathrm{~g}^{-1}\right)$, but the retention of the next cycles is much higher. In the following cycles, while there is some capacity fading, the reversible capacity stabilizes allowing A300 to achieve a capacity of $105 \mathrm{~mA} \mathrm{~h} \mathrm{~g}^{-1}$ after 100 cycles. A400 again shows the same trend as A300, but with a much lower initial capacity of $85 \mathrm{~mA} \mathrm{~h} \mathrm{~g}^{-1}$. After 100 cycles, A400 achieves only $65 \mathrm{~mA} \mathrm{~h} \mathrm{~g}^{-1}$, the least of the 4 samples.

As the crystallinity increases, there is an initial decrease in capacity, associated with the loss of surface area. The crystallinity has only increased by $\sim 4 \%$, while the surface area has decreased by $\sim 30 \%$ as we move from the as-prepared material to that annealed at $200^{\circ} \mathrm{C}$. The significant loss of surface area is apparently the cause of the capacity loss, as the trend in the capacity follows the same as A0 implying that surface-based storage dominates in the lower temperature (lower crystallinity) nanoanatase. A300, however shows a $20 \%$ increase in crystallinity as compared to A0, and while the surface area has decreased significantly, it seems that in this case, the increased crystallinity makes up for the loss of surface area pointing to the increasing importance of diffusion-based storage. Further, with the change in the trend of capacity, there is an apparent difference between the lithiation of A0 and A300. In contrast to A300, A400 shows limited increases in crystallinity, with a major loss in surface area. The capacity trend follows that of A300, but with the loss in surface area, the capacity decreases significantly.

As A0 and A300 showed similar capacities after 100 cycles at 10C, they were compared further in terms of rate capability (Figure 5.4a). The first 10 cycles (at 1C) show that $\mathrm{A} 0$ has a slightly higher formation cycle reversible capacity $\left(195 \mathrm{~mA} \mathrm{~h} \mathrm{~g}^{-1}\right)$ compared to A300 $\left(189 \mathrm{~mA} \mathrm{~h} \mathrm{~g}^{-1}\right)$. However, after the 3 rd cycle, A300 has a higher reversible capacity as it undergoes less capacity fading at low rate compared to A0. At 2C, A300 maintains, slightly, a higher capacity as compared to $\mathrm{A} 0$. However, at $5 \mathrm{C}, \mathrm{A} 0$ shows a higher capacity than $\mathrm{A} 300$. Interestingly, the difference between $\mathrm{A} 300$ and $\mathrm{A} 0$ at both $2 \mathrm{C}$ and $5 \mathrm{C}$ is $5 \mathrm{~mA} \mathrm{~h} \mathrm{~g}^{-1}$. As the specific 


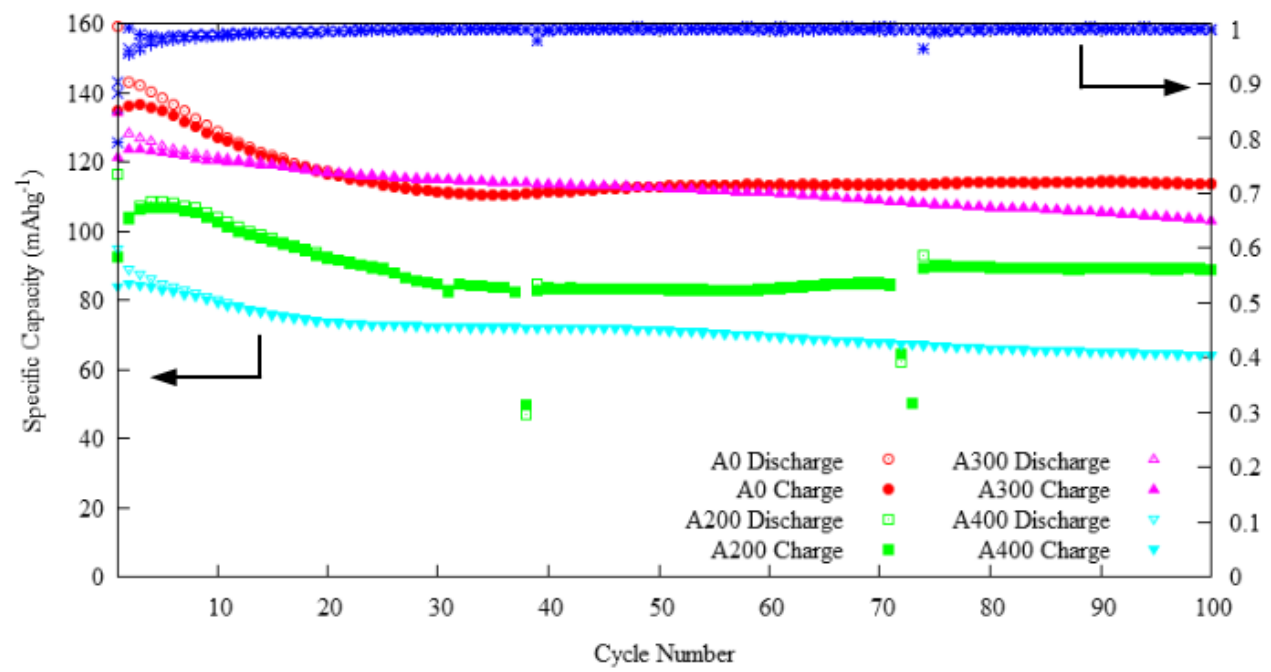

Figure 5.3: Specific capacity and coulombic efficiency data for all samples cycled 100 times at $10 \mathrm{C}$ rate $\left(\mathrm{C}=168 \mathrm{~mA} \mathrm{~h} \mathrm{~g}^{-1}\right)$ between 3 and $1 \mathrm{~V}^{1}$

current increases, the difference in capacity increases further to $50 \mathrm{~mA} \mathrm{~h} \mathrm{~g}^{-1}$ at $20 \mathrm{C}$. However, when the current is brought back down to $1 \mathrm{C}$, A300 again has a higher capacity than A0. The shift in capacity difference indicates that there is some difference in the storage mechanism that the two nanoanatase materials lithiate/delithiate. A0 and A300 were cycled at $1 \mathrm{C}$ rate 100 times to compare low rate performance (data shown in Figure S3). As observed in the rate capability study, here again there is a much smaller initial capacity drop, followed by consistently higher capacity after the third cycle for the A300 crystalline anatase material in comparison to the poorly-crystalline as-prepared A0 nanoanatase. While the increase in capacity is not significant, as cycle numbers increase, the difference in capacity increases showing a higher capacity retention as compared to A0. The higher capacity is likely due to increased diffusion-based lithiation. As the rate increases, the mechanism associated with the lithiation of A0 is less rate-limited apparently due to predominant surface-based storage. There exists a rate threshold (approximately occurring at $4 \mathrm{C}$ rate as shown in Figure $\mathrm{S} 4$ ) at which $\mathrm{A} 0$ and 
A300 nanoanatase materials achieve the same reversible capacity, although their mechanisms of Li-ion storage appear to differ with $\mathrm{A} 0$ dominated by surface storage (pseudocapacitive) and A300 by diffusion storage (intercalation).

By examining the voltage profiles of $\mathrm{A} 0$ and $\mathrm{A} 300$ at each rate (Figures $5.4 \mathrm{~b}$ and 5.4c), the mechanisms associated with lithium storage can be deduced [14]. The diffusion-based lithiation (intercalation) of anatase occurs in multiple steps $[20,25]$. In order to understand the various mechanisms involved in the process we focus on the plateau and tail of the voltage profile. In Figures $5.4 \mathrm{~b}$ and $5.4 \mathrm{c}$, the voltage profile at low rate has a voltage drop to $1.75 \mathrm{~V}$, where the $\mathrm{Li}$-ions insert into the tetrahedral interstitial sites forming a solid solution of $\alpha-\mathrm{Li}_{0.1} \mathrm{TiO}_{2}$ [20]. At $1.75 \mathrm{~V}$ a plateau occurs representative of bi-phasic diffusion within the $\mathrm{TiO}_{2}$ crystal structure. Here, the anatase undergoes a shift from tetragonal $\mathrm{TiO}_{2}$ to orthorhombic $\beta-\mathrm{Li}_{0.5} \mathrm{TiO}_{2}[18,20]$, as the Li-ions diffuse from the tetrahedral to the octahedral sites in the anatase. The diffusion processes end with a sloping tail until $1.5 \mathrm{~V}$, which is indicative that $\beta-\mathrm{Li}_{0.5} \mathrm{TiO}_{2}$ is the stable structure [20]. Below $1.5 \mathrm{~V}$ the storage occurs in the surface sites [21]. Upon charging (delithiation), the surface sites are the first to delithiate, indicated by the curved region up to $2 \mathrm{~V}$, where another diffusion plateau occurs as Li-ions are removed from the lattice once again. In both of these cases, the amount of capacity attributed to surface storage will be dictated by the surface sites available based on the surface area of the particles [38].

The voltage profile for $\mathrm{A} 0$ has a short plateau during its lithiation, even at low current, as compared to A300, which has half of its profile occurring in the plateau. As previously mentioned, the plateau indicates diffusion-based intercalation storage. When comparing the two, the majority of the storage in $\mathrm{A} 0$ comes from surface area-controlled mechanisms, while in A300 it is based primarily on diffusion control. While intuitively it can be assumed that this has to do with the difference

\footnotetext{
${ }^{1}$ Dark blue data points represent Coulombic efficiency. In this case all samples show almost $100 \%$ efficiency after the first 3 to 4 cycles.
} 

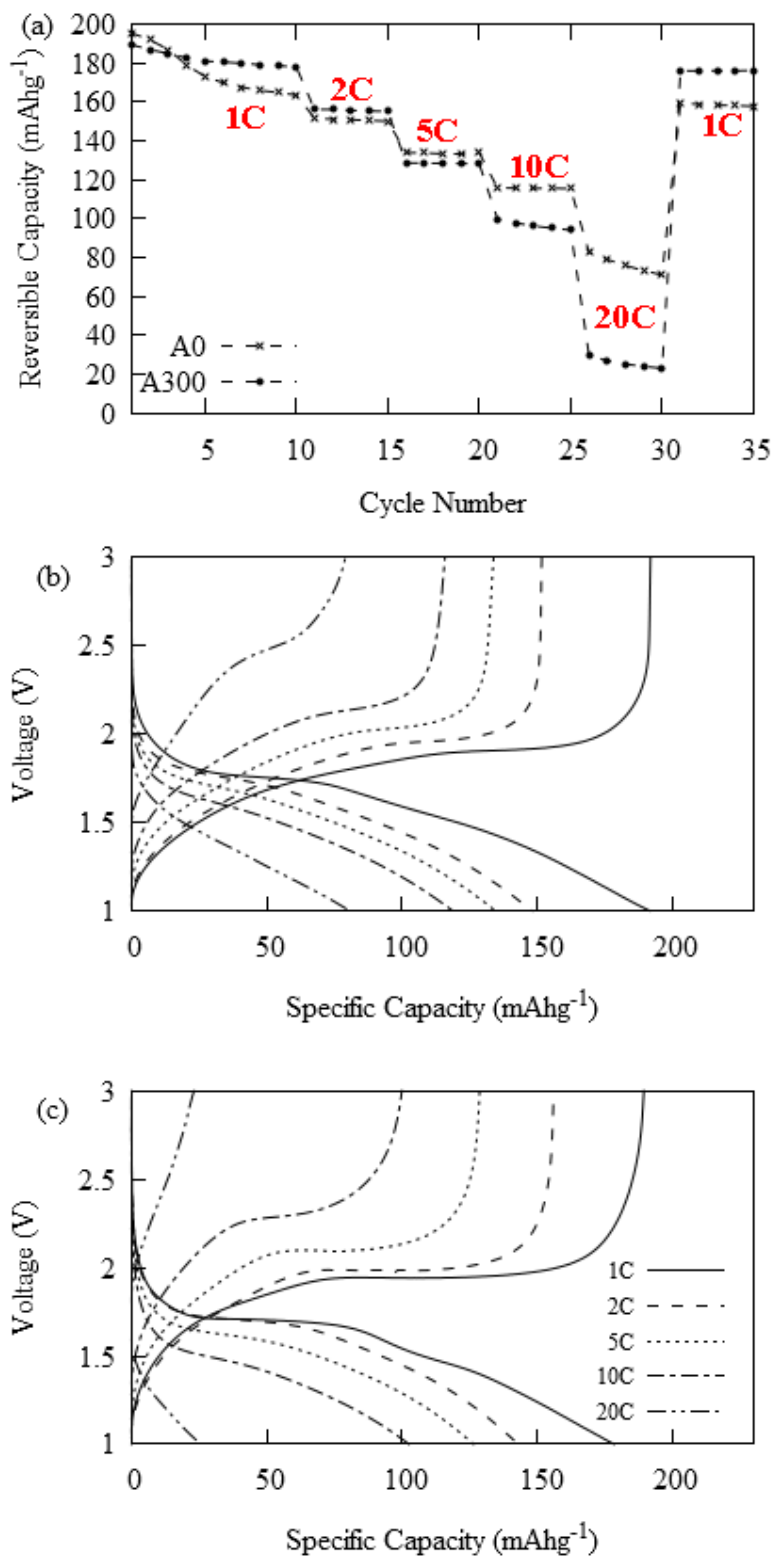

Figure 5.4: Rate capability data for $A 0$ and $A 300$ showing the reversible capacity at $1 \mathrm{C}, 2 \mathrm{C}, 5 \mathrm{C}, 10 \mathrm{C}, 20 \mathrm{C}$ and back at $1 \mathrm{C}$ (a) and the corresponding voltage profiles for $\mathrm{A} 0(\mathrm{~b})$ and $\mathrm{A} 300$ (c). 
in both crystallinity and surface area between the two, it is pertinent to examine the difference in diffusivity between A0 and A300 at various points in the lithiation to understand the dependence of capacity on the storage mechanism.

\subsubsection{Elecrtrochemical Impedance Spectroscopy.}

The diffusivity of $\mathrm{Li}^{+}$in $\mathrm{TiO}_{2}$ was determined through electrochemical impedance spectroscopy (EIS). After cycling 5 times at $1 \mathrm{C}$ rate, the electrodes were lithiated to various states of charge (SOC) corresponding to $0,25,50,75$, and $100 \%$ capacity. This capacity translates to a fraction $\mathrm{x}$ in $\mathrm{Li}_{\mathrm{x}} \mathrm{TiO}_{2}$ corresponding respectively to $\mathrm{x}=0,0.125,0.25,0.375$, and 0.5 . The impedance was measured from $1 \mathrm{MHz}$ to $0.5 \mathrm{mHz}$, after the cell had reached the open circuit voltage (OCV). The Nyquist plots for A0 and A300 at the various stages of lithiation can be seen in Figure 5.5. The Nyquist plots can be broken down into two parts, a semi-circular part and a linear part. The spectra exhibit either two semi-circles or a depressed semi-circle between $1 \mathrm{MHz}$ and $4 \mathrm{~Hz}$. This is indicative of two time constants, as shown by the equivalent circuit in Figure S4. Based on the values associated with the capacitance and resistance of the first time constant, the two semi-circles can coalesce and form a single, depressed semicircle. From $4 \mathrm{~Hz}$ to $0.5 \mathrm{mHz}$, the spectra exhibit a linear tail. This is representative of the Warburg impedance $\left(Z_{W}\right)$, which translates directly to the diffusivity of $\mathrm{Li}^{+}$into $\mathrm{TiO}_{2}$.

The equivalent circuit in Figure S5 was used to fit the spectra in Figure 5.5. $R_{e}$ corresponds to the electrolyte resistance, but also includes the uncompensated bulk resistances from the separator and counter electrode. The $C_{1} R_{1}$ parallel element has been explained to represent the SEI film, electrode roughness, and inhomogeneity of the lithiation reaction on the surface for both $\mathrm{TiO}_{2}$ and other materials $[30,39]$. However, SEI formation in $\mathrm{TiO}_{2}$ does not normally occur for cutoff voltages above $1 \mathrm{~V}$ and are a result of the decomposition of the electrolyte [25]. Instead, the roughness and inhomogeneity of lithiation are represented by the 

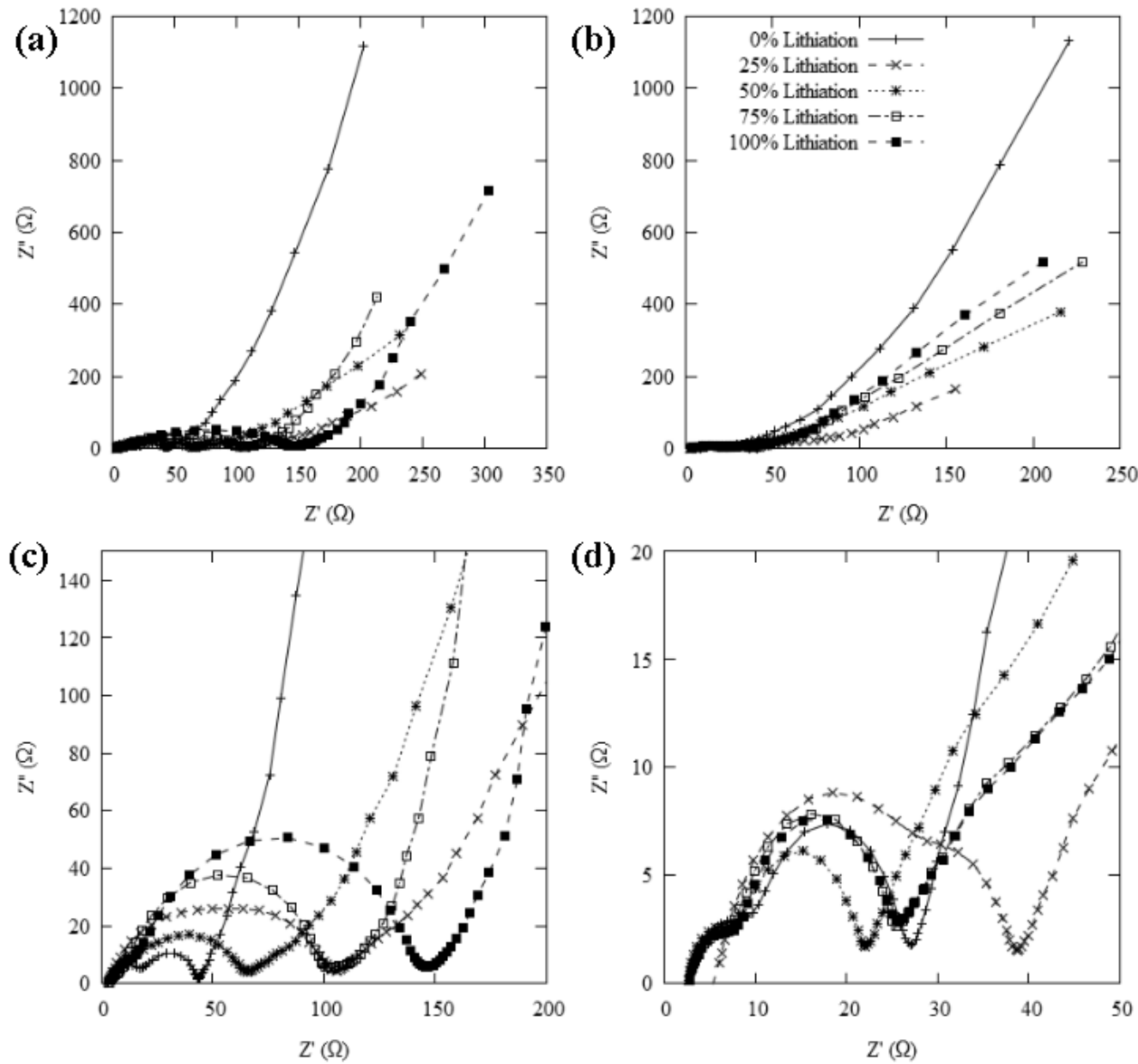

Figure 5.5: Nyquist plots for $A 0$ (a) and A300 (b) at 0, 25, 50, 75, and 100\% lithiation. Zoomed in view of high frequency impedance for A0 (c) and $\mathrm{A} 300(\mathrm{~d}){ }^{2}$ 
parallel element. The other parallel element is a Randall circuit comprised of the double layer capacitance, $C_{d} l$, the charge transfer resistance, $R_{c} t$, and the Warburg impedance, $Z_{W}$. This circuit describes the two time constants (two semicircles) and linear tail displayed in the EIS spectra.

A table of the values associated with the various circuit elements (Table S1) can be found in the supporting information along with plots of the trends in the data throughout the lithiation of both $\mathrm{A} 0$ and $\mathrm{A} 300$ (Figure S6). For both $\mathrm{A} 0$ and $\mathrm{A} 300$, $R_{e}$ remains essentially constant. This is expected as the electrolyte and separator remain constant, and the counter electrode is lithium metal, thus is considered an infinite lithium supply, and therefore will not have major resistance fluctuation. The capacitive part of the $C_{1} R_{1}$ element remains constant as well, in contrast the resistive portion of the element undergoes a significant increase from $0 \%$ lithiation to $25 \%$ lithation for both $\mathrm{A} 0$ and $\mathrm{A} 300$. However, the resistance drops again at $50 \%$ lithiation, though A0 does not see as significant of a decrease as A300. Due to the inhomogeneity of the crystal structure, there are fewer distinct lithiation zones (points) developing during the diffusion-based storage in the bi-phasic plateau of the voltage profile. At $50 \%$ lithiation, the resistance has decreased for both samples but A0 decreases only slightly. This may be again due to the lower crystallinity. As the lithiation mechanism shifts from diffusion-based to surface area control [21], the inhomogeneity still plays a significant role in the storage of lithium within the lattice. Only at $75 \%$ and full lithiation, where the majority storage occurs through surface area control, does the resistance here drop to its original level.

The charge transfer resistance and double layer capacitance of A0 and A300 show contrasting trends. For A300, charge transfer resistance is constant at all points during lithiation, the double layer capacitance, however, sees an initial drop, at $25 \%$ lithiation, which corresponds to the insertion of ions into the lattice, but then an exponential increase in capacitance at $75 \%$ lithiation. A0, on the other hand, has a major increase in charge transfer resistance at $75 \%$ lithiation and only a minor

\footnotetext{
${ }^{2}$ Frequency range for this data was between $1 \mathrm{MHz}$ and $0.5 \mathrm{mHz}$.
} 
increase in capacitance leading to a plateaued value at full lithiation. This indicates that for $\mathrm{A} 0$, there is pseudocapacitive storage in the surface layers, as indicated by the increased charge transfer resistance, most likely due to repulsive forces from $\mathrm{Li}-\mathrm{Li}$ interaction [40, 41]. For A300, however, the lattice more easily intercalates lithium within the fully developed crystalline structure, thus as it approaches the full extent of lithium insertion, the Li-ions would tend to align on the surface rather than inserting into surface layers. This causes a build up of charge, indicated by the increase in capacitance. However, it is likely that that charge is not entering the surface layers as the charge transfer resistance remains constant, thus with lower surface area, the contribution from pseudocapacitance decreases. In A0, because the ions can both insert into the disordered surface layers as well as align on the surface, the pseudocapacitive contribution is much greater, whereas for A300 is much lower due to reduced surface area.

The Warburg impedance describes the diffusion-based resistances within the electrode from which the Li-ion diffusion coefficient can be obtained [30]. The diffusivity of Li-ions in $\mathrm{TiO}_{2}$ was calculated using the equation derived by Ho, et al [42]:

$$
D_{L i^{+}}=\frac{1}{2}\left[\left(\frac{V_{M}}{S F A}\right)\left(\frac{d E}{d X}\right)\right]^{2}
$$

where $V_{M}$ is the molar volume of the active material, $S$ is the contact area between surface and electrolyte, $F$ is Faraday's constant, $A$ is a pre-exponential factor calculated from the Warburg impedance, and $d E / d x$ is the slope obtained from a plot of lithium content vs. OCV. The plots used to calculate the diffusion coefficient are seen in the Supporting Information of this paper. Figure S7a shows the plot of inserted lithium fraction $\left(\mathrm{x}\right.$ in $\mathrm{Li}_{\mathrm{x}} \mathrm{TiO}_{2}$ ) vs. OCV for the various lithiation states. The slope of the line provides the value of $d E / d x$ for $\mathrm{A} 0$ and $\mathrm{A} 300$. The pre-exponential factor, A, changes with state of charge and diffusivity so it was determined by plotting $\omega^{-1 / 2}$ vs $Z^{\prime \prime}$ (Figure S7b and S7c) for frequencies between 
10 and $0.5 \mathrm{mHz}$ at each of the various lithiation states for $\mathrm{A} 0$ and $\mathrm{A} 300$.

The important values and calculated diffusivity at each lithiation point are summarized in Table S2, while a plot of lithiation state vs. diffusivity for A0 and A300 can be seen in Figure S8. The initial diffusivity for both A0 and A300 is approximately $10^{-16} \mathrm{~cm}^{2} \mathrm{~s}^{-1}$. At $25 \%$ lithiation, the voltage profile shows that bi-phasic diffusion dominates. The diffusivity increases for both $\mathrm{A} 0$ and $\mathrm{A} 300$, however, A300 sees an increase of 2 orders of magnitude $\left(10^{-14} \mathrm{~cm}^{2} \mathrm{~s}^{-1}\right)$, while A0 only sees an increase of an order of magnitude $\left(10^{-15} \mathrm{~cm}^{2} \mathrm{~s}^{-1}\right)$. At $50 \%$ lithiation, both samples are no longer in the bi-phasic plateau, and diffusivity decreases significantly, and by $100 \%$ lithiation, the diffusivity is the same as at $0 \%$ lithiation.

With the increase in crystallinity (A300), there is an increase in diffusivity of Li-ions in anatase. This is in good agreement with Wang, et al [26], as the more ordered crystal can more easily change from tetragonal to orthorhombic. By increasing crystallinity, the ions diffuse more effectively as evidenced by the higher diffusion coefficient determined for A300, increasing the amount of possible storage due to bi-phasic diffusion. However, the reliance on diffusion as the major mechanism for charge storage poses a problem for cyclability as in more detail is discussed later. Figures $5.4 \mathrm{~b}$ and $5.4 \mathrm{c}$ show an increase in polarization between lithiation and delithiation. The polarization is caused by the activation energy associated with the change in crystal structure during the insertion and removal of Li-ions. As the current is higher (lithation and delithiation at faster rate) there is more resistance to phase change. At higher rate, as diffusion occurs, the voltage difference between lithiation and delithiation diffusion increases significantly.

\subsubsection{Diffusivity Effects on Pseudocapacitance.}

Having determined that enhanced crystallinity in A300 induced by annealing led to increased Li-ion diffusivity begs the question as to how this affects lithiation at different charging/discharging rate. Differential capacity $(D Q / D V)$ analysis 
allows for a more detailed investigation into the contributions of diffusion and surface area based storage to capacity by translating the plateaus associated with diffusion into peaks [14]. For a voltage profile showing pure diffusion-based storage, sharp peaks at the oxidation and reduction potentials will be seen while the rest of the plot will be approximately at zero. When $D Q / D V$ is greater than zero, but not a peak, it can be assumed that there is some sort of pseudocapacitive charge storage. In the case of $\mathrm{TiO}_{2}$ this happens in the voltage range after reduction and before oxidation. Figure 5.6 shows the $D Q / D V$ curves for $\mathrm{A} 0$ and $\mathrm{A} 300$ at various rates, as well as a comparison between the two at $1 \mathrm{C}$ and $10 \mathrm{C}$. Comparing the curves in Figures 5.6a and 5.6b, we note the peaks, which correspond to diffusion-based capacity are extremely tall for A300, and that pseudocapacitive storage is almost non-existent. Further, as the rate increases, the distance between the peaks increases, representing an increase in polarization between intercalation and deintercalation. While both $\mathrm{A} 0$ and $\mathrm{A} 300$ have intercalation and deintercalation peaks at approximately 1.7 and $2.0 \mathrm{~V}$, respectively, at $1 \mathrm{C}$ rate, A0 sees only $0.2 \mathrm{~V}$ overall increase in polarization at $10 \mathrm{C}$, while the increase for $\mathrm{A} 300$ is $0.6 \mathrm{~V}$.

When a closer, side-by-side examination of the $D Q / D V$ curves for A0 and A300 at $1 \mathrm{C}$ rate is performed (Figure 5.6c), a second peak can be seen at $1.5 \mathrm{~V}$. While the peak is just a hump for A0, it is clearly seen in A300. This hump is associated with the insertion of lithium as the stable $\beta-\mathrm{Li}_{0.5} \mathrm{TiO}_{2}$ phase, as translated from the voltage profile. Theoretically, this would be followed by another plateau where lithium would insert as a second bi-phasic region, this time consisting of $\beta-\mathrm{Li}_{0.5} \mathrm{TiO}_{2}$ and $\gamma-\mathrm{LiTiO}_{2}$ [20]. However, as the $D Q / D V$ plots show, this stable single phase diffusion is followed by surface storage. At $1 \mathrm{C}$, the capacitive contribution to capacity is approximately the same during the lithiation. During delithiation, there is less capacitive contribution in A300, leading to the sharp peak seen in Figure 5.6a (Figure 5.6c has been truncated to show the capacitive contributions). Interestingly, the polarization between intercalation and deintercalation is smaller for A0 than A300, even at low rate, which may be explained by the lower contribution of 
diffusive (intercalation type) storage. By relying less on lithium diffusion, and instead storing in surface sites, A0 does not undergo a large-scale phase change, therefore experiencing less resistance.

Further, at $10 \mathrm{C}$, there is a stark contrast between the $D Q / D V$ curves of the $\mathrm{A} 0$ and A300 nanoanatase materials (Figure 5.6d). Aside from the increased polarization, the contribution from pseudocapacitive storage is almost zero for A300, especially during deintercalation. A300, however, has a higher diffusion peak in the deintercalation half of the curve. It is postulated that because of decreased surface area, A300 has fewer sites for pseudocapacitive storage; as a consequence the bulk of its reversible capacity comes from diffusion-based storage. However, with the insertion of lithium into the lattice, and the phase-change associated with that insertion, the stresses on the crystal structure of A300 may augment significantly more than in the case of A0 that relies mainly on pseudocapacitive storage, an issue that can impact the material's cyclability as discussed next.

\subsubsection{Impact of Mode of Storage on Cyclability.}

In order to determine the effect of mode of storage on nanoanatase crystal structure and capacity retention, A0 (predominantly surface-based storage) and A300 (predominantly diffusion-based storage) were cycled at 10C for 500 cycles. Figure 5.7 shows that for the 1 st 100 cycles, A0 and A300 exhibit macroscopically similar capacity retention behaviour. A300 shows a stable capacity that is slightly higher than A0, then decreases to just below it. After 100 cycles, however, the capacity of A300 drops dramatically until it reaches a capacity of approximately $45 \mathrm{~mA} \mathrm{~h} \mathrm{~g}^{-1}$. A0, however, remains constant until approximately 175 cycles after which its capacity decreases slowly but always remaining higher than that of A300 (70 $\mathrm{mA} \mathrm{h} \mathrm{g}^{-1}$ vs $45 \mathrm{~mA} \mathrm{~h} \mathrm{~g}^{-1}$ ) 

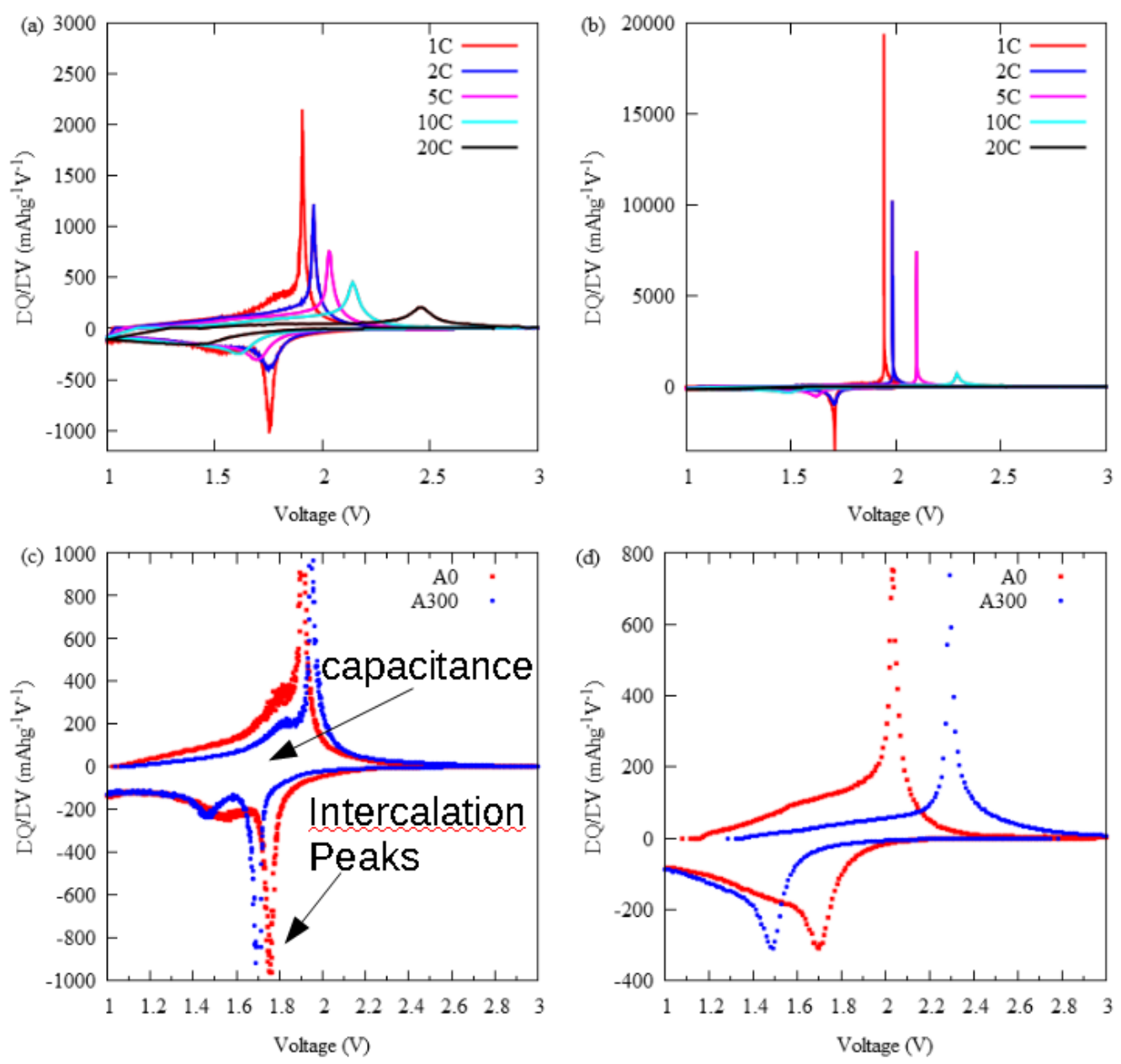

Figure 5.6: Differential capacity (DQ/DV) plots of $A 0(a)$ and $A 300$ (b) at $1 \mathrm{C}, 2 \mathrm{C}$, $5 \mathrm{C}, 10 \mathrm{C}$, and $20 \mathrm{C}$. Comparison of $\mathrm{DQ} / \mathrm{DV}$ of $\mathrm{A} 0$ and $\mathrm{A} 300$ at $1 \mathrm{C}(\mathrm{c})$ and $10 \mathrm{C}(\mathrm{d})$ rates. 


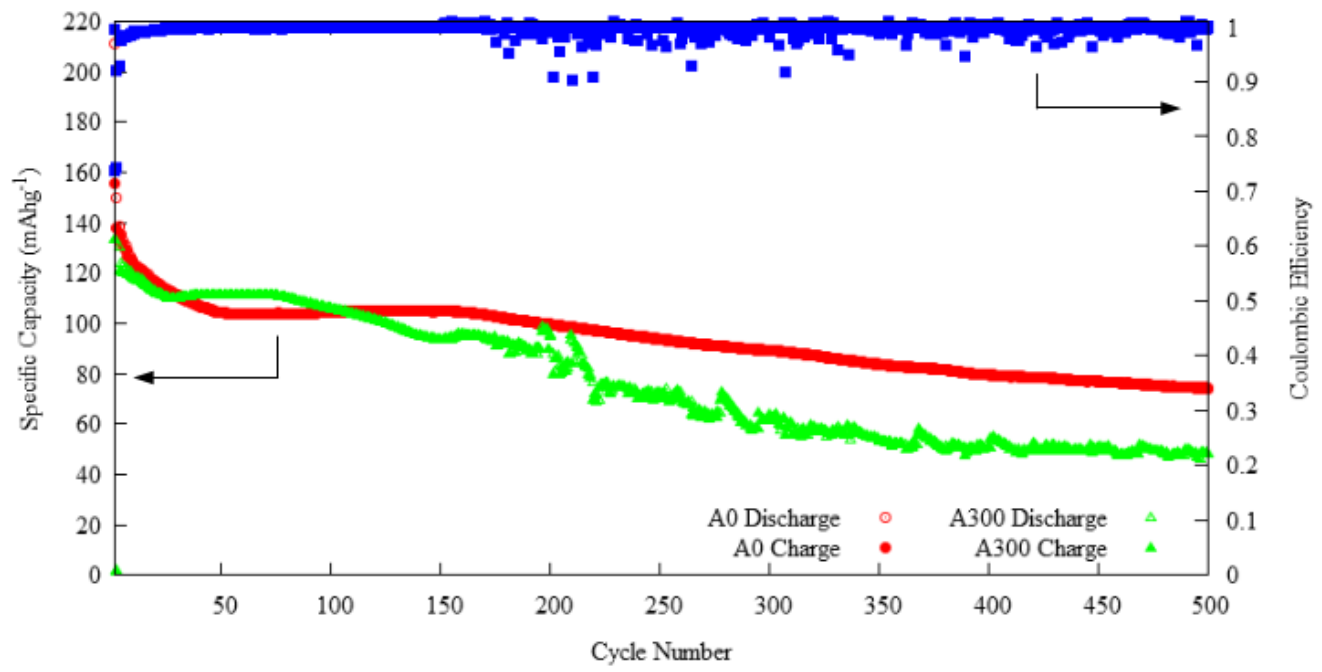

Figure 5.7: Specific capacity and coulombic efficiency for A0 and A300 lithiated at $10 \mathrm{C}$ rate $\left(1 \mathrm{C}=168 \mathrm{~mA} \mathrm{~g}^{-1}\right)$ over 500 cycles. 
The breakdown in capacity exhibited by the A300 material may be linked to the increased crystallinity combined with the loss of surface area. In both A0 and A300, the differential voltage profiles (Figures S8a and S8b) change significantly over 500 cycles. Where the first 100+ cycles show a distinct diffusion plateau, though short due to the high current and fast Li-ion intercalation and deintercalation transport, the plateau eventually disappears for both materials and the curves assume the characteristic shape of essentially sole pseudocapacitive type of storage $[14,15]$. In the case of A300, the lower surface area leads to low storage at the end of 500 cycles, while A0 retains a majority of its capacity. The question still remains as to why this capacity loss occurs. As a first hypothesis it was thought this stems from a structural change that blocks Li-ion diffusion channels. However, XRD patterns of $\mathrm{A} 0$ and $\mathrm{A} 300$ before and after 500 cycles (Figure S10) show that there is only a minor peak shift for both samples. This could be associated with small changes in lattice parameter related to some (but not all) irreversible capacity loss. As no significant structural change was detected the other hypothesis made to explain the capacity loss relates to possible interaction with the electrolyte.

It was seen in Figure 5.6b and 5.6c that with increased rate, there is a large increase in polarization. The resistance brought on by the increased rates (a larger amount seen in A300 as compared to A0) may cause localized voltage drops to below the $1 \mathrm{~V}$ cutoff voltage. As explained by Bresser, et al., a voltages below $1 \mathrm{~V}$ leads to passivation layer formation due to interaction with the electrolyte [25]. Because of this apparent voltage instability, the electrolyte becomes more reactive and catalyzes the formation of a passivation layer, whose presumably increasing thickness is responsible for the increased capacity fading. The same phenomenon is seen during the rate capability testing when the rate is increased to $20 \mathrm{C}$. Even though it is only for 5 cycles, the polarization is so large that a $20 \mathrm{~mA} \mathrm{~h}^{-1}$ capacity fade from the 1 st to the 5 th cycle occurs, however, upon returning to $1 \mathrm{C}$, the polarization is essentially removed, and the capacity fading is negligible. This phenomenon is seen only at high rate because of the large increase 
in polarization associated with attempting to diffuse lithium into the crystal. This is why the capacity loss for A0 is only approximately $30 \mathrm{~mA} \mathrm{~h} \mathrm{~g}^{-1}$ as compared to $60 \mathrm{~mA} \mathrm{~h} \mathrm{~g}^{-1}$ for A300. By taking advantage of pseudocapacitance, A0 exhibits much less polarization as it relies less on diffusion-based storage. Furthermore by lowering the charging/discharging rate polarization is reduced hence avoiding the drastic capacity loss due to electrolyte interaction. This was demonstrated by cycling the A0 material for 500 times but this time at $2 \mathrm{C}$. As can be seen with the cycling data plotted in Figure S11, a much more stable capacity retention is achieved with only a modest capacity fade $\left(\sim 20 \mathrm{~mA} \mathrm{~h} \mathrm{~g}^{-1}\right)$ linked to typical coulombic efficiency behaviour. Further alleviation of the capacity fading problem may be achieved by cycling to a cutoff voltage of $1.2 \mathrm{~V}$, as described by Bresser, et al. [25]. Alternatively, fabrication of an anatase electrode structures that maximize the benefits of increased surface area and crystallinity without sacrificing one for the other may provide a stable and highly reversible Li-ion storage performance.

\subsection{Conclusions}

A new variety of phase pure nano-anatase $\mathrm{TiO}_{2}$ material has been synthesized via a scalable aqueous solution process carried out in a continuous-stirred tank reactor at $80^{\circ} \mathrm{C}$. The anatase has a small nanocrystallite size $(\sim 6 \mathrm{~nm})$ and large surface area $\left(222.6 \mathrm{~m}^{2} \mathrm{~g}^{-1}\right)$, which translates to an excellent candidate for high rate lithiation and delithiation performance. As-synthesized anatase (A0) provided a stable reversible capacity at $1 \mathrm{C}\left(\mathrm{C}=168 \mathrm{~mA} \mathrm{~g}^{-1}\right)$ rate, achieving $150 \mathrm{~mA} \mathrm{~h} \mathrm{~g}^{-1}$ over 100 cycles. By annealing, crystallinity was increased, while surface area decreased allowing for the investigation of the contributions of each toward lithiation and delithiation capacity. A0 and the annealed samples were cycled at high rate (10C) over 100 cycles, where $\mathrm{A} 0$ achieved $115 \mathrm{~mA} \mathrm{~h}^{-1}$ and the sample annealed at $300^{\circ} \mathrm{C}(\mathrm{A} 300)$ achieved the highest reversible capacity (of the annealed samples) of $105 \mathrm{~mA} \mathrm{~h} \mathrm{~g}^{-1}$. When these two samples were compared at various rates, it was found that at low 
rate ( $1 \mathrm{C}$ and $2 \mathrm{C}) \mathrm{A} 300$ had a higher reversible capacity than $\mathrm{A} 0$, due to increased diffusion-based storage as the major mechanism for Li-ion transport. At high rate however, A0 had much higher capacity due to the increased surface area and ability to store Li-ions pseudocapacitively.

Using electrochemical impedance spectroscopy, it was determined that with increased crystallinity, the diffusivity of Li-ions within the lattice increases by an order of magnitude resulting in A300's reliance mainly on diffusion-based intercalation as storage mechanism. As rate increases, the increased crystallinity and decreased surface area causes increased polarization. This manifests in long-term stability loss due to apparent electrolyte reactivity and breakdown. By understanding the effects of anatase crystallinity and surface area on Li-ion storage, the electrode fabrication process can be optimized to provide the best combination of properties for highly stable and reversible performance.

\section{Acknowledgements}

The authors would like to thank Dr. Fuqiang Guo for his help with the synthesis tests, and Dr. Mert Celikin for his help with TEM micrographs. This research is supported with a strategic project grant from the Natural Sciences and Engineering Research Council of Canada (NSERC) and sponsored by Hydro-Quebec's Energy Conversion and Storage department.

\subsection{References}

[1] Z. Yang, D. Choi, S. Kerisit, K. M. Rosso, D. Wang, J. Zhang, G. Graff, J. Liu, J. Power Sources, 192, 588 (2009).

[2] C. Jiang, J. Zhang, J. Mater. Sci. Technol., 29, 97 (2013).

[3] Y. Yang, H. Wang, Q. Zhou, M. Kong, H. Ye, G. Yang, Nanoscale, 5, 10267 (2013). 
[4] J. S. Chen, X. W. Lou, J. Power Sources, 195, 2905 (2010).

[5] Y.-S. Hu, L. Kienle, Y.-G. Guo, J. Maier, Adv. Mater., 18, 1421 (2006).

[6] S. K. Panda, S. Lee, W.-S. Yoon, H. Shin, J. Power Sources, 249, 59 (2014).

[7] X. Su, Q.-L. Wu, X. Zhan, J. Wu, S. Wei, Z. Guo, J. Mater. Sci., 47, 2519 (2012).

[8] D. Dambournet, I. Belharouak, K. Amine, Chem. Mater, 22, 1173 (2010).

[9] A. R. Armstrong, G. Armstrong, J. Canales, R. Garcia, P. G. Bruce, $A d v$. Mater., 17, 862 (2005).

[10] R. Giannuzzi, M. Manca, L. De Marco, M. R. Belviso, A. Cannavale, T. Sibillano, C. Giannini, P. D. Cozzoli, G. Gigli, ACS Appl. Mater. Interfaces, 6, 1933 (2014).

[11] J. Wang, J. Polleux, J. Lim, B. Dunn, J. Phys. Chem. C, 111, 14925 (2007).

[12] K. Zhu, Q. Wang, J. H. Kim, A. A. Pesaran, A. J. Frank, J. Phys. Chem. C, 116, 11895 (2012).

[13] B. Laskova, M. Zukalova, A. Zukal, M. Bousa, L. Kavan, J. Power Sources, 246, 103 (2014).

[14] A. G. Dylla, G. Henkelman, K. J. Stevenson, Acc. Chem. Res., 46, 1104 (2013).

[15] V. Augustyn, P. Simon, B. Dunn, Energy Environ. Sci., 7, 1597 (2014).

[16] L. Kavan, J. Solid State Electrochem., Accepted (2014).

[17] T. Brezesinski, J. Wang, J. Polleux, B. Dunn, S. H. Tolbert, J. Am. Chem. Soc., 131, 1802 (2009).

[18] R. van de Krol, A. Goossens, A. A Meulenkamp, J. Electrochem. Soc., 146, 3150 (1999).

[19] W. J. H. Borghols, D. Lutzenkirchen-Hecht, U. Haake, E. R. H. van Eck, F. M. Mulder, M. Wagemaker, Phys. Chem. Chem. Phys., 11, 5742 (2009). 
[20] A. A. Belak, Y. Wang, A. Van der Ven, Chem. Mater, 24, 2894 (2012).

[21] C. Jiang, M. Wei, Z. Qi, T. Kudo, I. Honma, H. Zhou, J. Power Sources, 166, 239 (2007).

[22] J. W. Kang, D. H. Kim, V. Mathew, J. S. Lim, J. H. Gim, and J. Kim, J. Electrochem. Soc., 158, A59 (2011).

[23] J. S. Chen, X. W. Lou, Electrochem. Commun., 11, 2332 (2009).

[24] H.-W. Shim, D. K. Lee, I.-S. Cho, K. S. Hong, D.-W. Kim, Nanotechnology, 21, 255706 (2010).

[25] D. Bresser, E. Paillard, E. Binetti, S. Krueger, M. Striccoli, M. Winter, S. Passerini, J. Power Sources, 206, 301 (2012).

[26] J. Wang, Y. Zhou, Y. Hu, R. O'Hayre, Z. Shao, J. Mater. Sci. 48 (2013) 2733-2742.

[27] W. Li, F. Wang, S. Feng, J. Wang, Z. Sun, B. Li, Y. Li, J. Yang, A. A. Elzatahry, Y. Xia, D. Zhao, J. Am. Chem. Soc. 135, 18300 (2013).

[28] C. Charbonneau, R. Gauvin, G. P. Demopoulos, J. Electrochem. Soc., 158, H224 (2011).

[29] A. Yasin, F. Guo, G. P. Demopoulos, Materials Research Society Spring Meeting (2013).

[30] H. Joachin, T. D. Kaun, K. Zaghib, J. Prakash, J. Electrochem. Soc., 156, A401 (2009).

[31] R. Delhez, T. H. Keyser, J. I. Langford, D. Louer, E. J. Mitmeijer, E. J. Sonneveld in: R. A. Young (Ed.), The Reitveld Method, IUCr Monograph \#5, Oxford University Press, 132 (1995).

[32] M. Wagemaker, W. J. H. Borghols, F. M. Mulder, J. Am. Chem. Soc., 129, 4323 (2007).

[33] E. Hosono, T. Kudo, I. Honma, H. Matsuda, H. Zhou, Nano Lett. 9, 1045 (2009). 
[34] D. Zhan, Q. Zhang, X. Hu, G. Zhu, T. Peng, Solid State Ionics, 239, 8 (2013).

[35] G. Sudant, E. Baudrin, D. Larcher, J.-M. Tarascon, J. Mater. Chem. 15 (2005) 1263-1269.

[36] H.-G. Jung, S. W. Oh, J. Ce, N. Jayaprakash, Y.-K. Sun, Electrochem. Commun., 11, 756 (2009).

[37] F. Zhang, Y. Zhang, S. Song, H. Zhang, J. Power Sources, 196, 8618 (2011).

[38] M. J. Sussman, N. Brodusch, R. Gauvin, G. P. Demopoulos, J. Electrochem. Soc., 160, A3100 (2013).

[39] J. Wang, Y. Zhou, Y. Hu, R. O'Hayre, Z. Shao, J. Phys. Chem. C, 115, 2529 (2011).

[40] A. G. Dylla, P. Xiao, G. Henkelman, K. J. Stevenson, J. Phys. Chem. Lett. 3, 2015 (2012).

[41] M. J. Sussman, M. Celikin, A. Yasin, G. P. Demopoulos, Electrochim. Acta (Submitted).

[42] C. Ho, I. D. Raisrick, R. A. Huggins, J. Electrochem. Soc., 127, 343 (1980). 



\section{Chapter 6}

\section{Engineering 3-D Li-Ion Electrodes with Enhanced Charge Storage Properties based on Solution-Processed and Sintered Anatase Nanocrystal-Carbon Mesoporous Structures}

In chapter 3, a fabrication method developed in order to remove non-electroactive binders and disperse $\mathrm{TiO}_{2}$ and carbon was developed. In Chapter 5, anatase synthesized using aqueous solution processing was characterized electrochemically. In this chapter the use of aqueous anatase to build hierarchically porous 3-dimensional electrodes is investigated. By using dispersants and controlled sintering, a percolating carbon network, as well as increased crystallinity and inter-particle necking are built into the electrodes. Controlling the sintering allows the particles to neck and become more crystalline without a significant drop in surface area, leading to higher capacity at low rate (1C), and comparable capacity at high rate (10C). This chapter is currently submitted, as a letter, for publication: M. J. Sussman, N. Brodusch, R. Gauvin, G. P. Demopoulos, Engineering 3-D Li-Ion Electrodes with Enhanced Charge Storage Properties based on Solution-Processed and Sintered Anatase Nanocrystal-Carbon Mesoporous Structures, ACS Sustainable Chemistry \& Engineering, under manuscript ID: sc-2014-00739t). The letter format does not follow the traditional sub-sections as seen in previous chapters. 


\subsection{Abstract}

3-Dimensional electrodes are an excellent platform for enhancing the electrochemical properties of Li-ion batteries, but fabrication involves complex, difficult to scale processing. Here, anatase nanocrystallites $(\sim 6 \mathrm{~nm})$ were used as active material in a fabrication process employing $450{ }^{\circ} \mathrm{C}$ sintering of an ethanolic paste to engineer a mesoporous 3-D structure of interconnected particles. These electrodes provide a percolating carbon network with enhanced conductivity and annealing leading to high, stable charge storage characteristics. The electrodes provide greater capacity ( $\sim 27 \%$ ) compared to binder-based electrodes and provide a platform for an easily scalable process applicable to various active materials.

\subsection{3-D Anatase Electrodes}

Commercially, electrodes for lithium ion batteries are fabricated using a paste of particulate active insertion material, conductive carbon, and a polymeric binder [2], usually poly(vinylidene) fluoride (PVDF) in an n-methyl 2-pyrrolidone (NMP) solvent. The porous electrode structure is obtained by applying the paste as a film onto the metallic current collector, drying at $120^{\circ} \mathrm{C}$ and pressing. Avoidance of harsh chemicals like NMP has motivated researchers to investigate alternative, environmentally friendly polymers, such as carboxymethyl cellulose, styrene butadiene rubber and others, which are soluble in water [1, 3--5]. However, these binders still add non-electroactive mass to the electrode, reducing its specific energy. Another problem of the fabrication process is that the resultant particle-carbon-binder composite does not achieve full ionic and electronic conductivity properties due to poor particle-carbon networking [6]. This has prompted research into alternative fabrication methods not involving particles or binders. Monolithic and other binder-free electrodes providing a 3-dimensional electrode structure show promise for use as both anode and cathode [7--9] but typically are not amenable to large 
scale manufacturing.

Previous efforts toward fabricating 3-D $\mathrm{TiO}_{2}$ electrodes showed similar limitations. Nanostructured $\mathrm{TiO}_{2}$, particularly its anatase polymorph, is recognized as an excellent substitute for graphite in anodes due to non-toxicity, safety and high rate capability [10], suggesting utility in 3-D electrode fabrication using various techniques like titanium anodization, sputtering, templating, and electrospinning [11--15]. Of these methods, the most notable is electrospinning of $\mathrm{TiO}_{2}$ fibers using an organic precursor solution followed by uniaxial pressing and calcining at $450^{\circ} \mathrm{C}$, as described by Lee et al. [13]. This fabrication process generated 3-D $\mathrm{TiO}_{2}$ electrodes with higher charge capacity (188 vs. $155 \mathrm{~mA} \mathrm{~h} \mathrm{~g}^{-1}$ after 100 cycles at $0.2 \mathrm{C}$ ) than conventionally fabricated electrodes, proving that the $3-\mathrm{D}$ electrode concept holds promise.

The proposed 3-D electrode fabrication techniques are both complex and costly to implement (requiring templates [12] or sputtering [15]), or make use of special nanostructures other than the nanoparticles and carbon black favored by industry. For example, electrospinning which yielded electrodes with the best properties, produces $\mathrm{TiO}_{2}$ nanofibers via a low throughput and expensive organic precursor solution method. Industry is being called on to provide new generation Li-ion batteries for the emerging electric vehicle transportation sector. As such, there is a need for cost-competitive and easily scalable synthesis and fabrication technologies for nanoparticle-based electrodes [16]. In response, we developed fully solution-processable 3-D electrode structures using screen printable nanoparticle pastes and sintering [17]. This template-free approach is demonstrated using nanoanatase anodes, but can easily be adapted for other anode or cathode nanoparticle materials. This anatase was recently evaluated in standard electrodes and found to store Li-ions via intercalation and pseudocapacitance, the relative contribution of each depending on the thermal treatment history of the anatase nanocrystals [18]. We demonstrate that by engineering the novel 3-D sintered electrodes, the anatase nanocrystalline particles deliver higher reversible storage capacity than in standard 
(PVDF binder-based) electrode configuration via induced inter-particle necking and optimized percolating carbon networking [19]. This fabrication method provides a platform for superior nanoparticle-based Li-ion battery electrodes employing easily scalable processing steps.

Standard electrodes were fabricated by mechanically mixing $6 \mathrm{~nm}$ anatase nanocrystallites, acetylene black, and PVDF in NMP to produce a paste with constitution $80 \mathrm{wt} \% / 10 \mathrm{wt} \% / 10 \mathrm{wt} \%$, respectively. The paste was deposited by doctor blading on aluminum foil and dried for 24 hours at $120^{\circ} \mathrm{C}$. The 3-D electrodes were fabricated using the method we developed and described earlier, following minor modifications [17]. 3-D electrodes had a composition of $90 \mathrm{wt} \%$ anatase and $10 \mathrm{wt} \%$ carbon. Details on anatase synthesis and electrode formulation can be found in Supporting Information. The paste was doctor bladed or screen printed on aluminum foil and subsequently annealed/sintered at $450{ }^{\circ} \mathrm{C}$ following a specific heating profile [20].

Physical characterization of the electrode structure was made via scanning electron microscopy (SEM), X-ray mapping, and BET surface area analysis. Electrochemical characterization was performed using Swagelok-type cells constructed in an argon-filled glovebox using lithium foil as the counter electrode. The electrolyte was $1 \mathrm{M} \mathrm{LiPF}_{6}$ in EC:DMC:DEC (2:2:1 by weight) mixed solvent (BASF). Galvanostatic charge and discharge was performed between 1 and $3 \mathrm{~V}$ at various C-rates $\left(1 \mathrm{C}=168 \mathrm{~mA} \mathrm{~g}^{-1}\right)$. Cycle life and rate capability was performed on all cells. Detailed experimental procedures can be found in Supporting Information (SI).

Secondary electron (SE) and Backscatter electron (BSE) micrographs of both standard and 3-D electrodes reveal stark differences that affect performance. The morphology of the anatase nanocrystallites can be evaluated using transmission electron microscopy, as shown in Figure F.1 of the SI. Figure 6.1 shows topographic and constitutional information of both electrode film types. Figure 6.1a-c and 6.1d-f are of the same regions in the standard and 3-D electrodes, respectively. The first 
significant difference is the size of aggregates (Figure 6.1a and 6.1d). The 3-D electrode has much smaller aggregates (Figure 6.1d), which can be attributed to the use of rheological dispersants (terpineol/ethyl cellulose) and ultrasonication. The nanocrystallites aggregate to form larger, mesoporous secondary particles. In the standard electrodes, as only mechanical mixing is employed, these aggregates are much larger. Investigating the composition in Figure $6.1 \mathrm{~b}$ and $6.1 \mathrm{e}$, where light colored regions represent $\mathrm{TiO}_{2}$ and dark colored regions represent carbon, the 3-D electrodes have uniform intermixing, as opposed to the significant segregation associated with the standard electrodes. Heat maps of the two types of electrodes are shown in Figure 6.1c and 6.1f. The heat maps were built by associating a color gradient to the black-to-white threshold gradient associated with the compositional images (Figure 6.1b and 6.1e). This allows colors to be associated with various features in the image, making it easier to interpret. White is associated with macroporosity in the electrode, red is associated with carbon, green with $\mathrm{TiO}_{2}$, and blue, mesoporosity in the $\mathrm{TiO}_{2}$ aggregates. Examining the heat maps in Figure 6.1c and 6.1f once more, it becomes evident that the carbon is homogeneously distributed through the $\mathrm{TiO}_{2}$ matrix in the case of the 3-D electrode, but not in the standard electrode. However, the micrographs in Figure 6.1 show only the surface of the electrodes. The excellent microstructure of the 3-D electrode can be further evaluated by examining its cross-section (Figure 6.2). As seen throughout the thickness of the film ( $\sim 60$ microns), carbon and $\mathrm{TiO}_{2}$ are uniformly distributed with carbon, providing a conductive percolating network. Finally, Figure F.4 shows a high magnification SE micrograph of the 3-D electrode that clearly reveals that sintering has induced inter-particle necking, resulting in a porous monolithic structure that provides physical support and improved interfacing with the electrolyte for enhanced Li-ion intercalation pathways. Pore size characterization (see SI) of the two electrode types confirmed the superior porous structure of the 3-D electrode. The average pore size in the 3-D film is double $(15 \mathrm{~nm})$ that of the binder-based film $(7.6 \mathrm{~nm})$. It is postulated, based on high-resolution SEM imaging, that the 
PVDF polymeric binder fills part of the pore space via surface attachment/coating, hence the smaller pore size, which consequently interferes with Li-ion shuttling at the particle/electrolyte interface.

Figure 6.3 shows the galvanostatic cycling at $1 \mathrm{C}\left(168 \mathrm{~mA} \mathrm{~g}^{-1}\right)$ over 100 cycles. In the initial cycle (Figure F.5), the standard electrode lithiates (discharge) to $x=0.68 \mathrm{in}_{\mathrm{x}} \mathrm{TiO}_{2}$, the equivalent of $229 \mathrm{~mA} \mathrm{~h} \mathrm{~g}^{-1}$, and has a charge (delithiation) capacity of $196 \mathrm{~mA} \mathrm{~h}^{-1}(x=0.58)$. The 3-D electrode, however, achieves $245 \mathrm{~mA} \mathrm{~h}^{-1}$ during lithiation and $219 \mathrm{~mA} \mathrm{~h} \mathrm{~g}^{-1}$ during delithiation $(x=0.73$ and 0.65 , respectively). This gives initial efficiencies of $85 \%$ and $90 \%$ for standard and 3-D electrodes, respectively. As the electrodes cycle, they both achieve nearly $100 \%$ Coulombic efficiency over 100 cycles, however, the 3-D electrode has a much higher capacity retention. The standard electrode loses $30 \mathrm{~mA} \mathrm{~h} \mathrm{~g}^{-1}$ of reversible capacity during the next 10 cycles, whereas the 3-D electrode loses only $20 \mathrm{~mA} \mathrm{~h} \mathrm{~g}^{-1}$. After 100 cycles, the reversible capacity of the standard electrode is approximately $145 \mathrm{~mA} \mathrm{~h} \mathrm{~g}^{-1}$, while the 3-D electrode retains approximately $185 \mathrm{~mA} \mathrm{~h} \mathrm{~g}^{-1}$ a $27 \%$ higher capacity retention.

Increased capacity can be explained by the better ionic and electronic conductivity properties of the 3-D porous electrode structure. As previously described by Bresser et al. [19], the improved nano $\mathrm{TiO}_{2} /$ carbon dispersion creates an effective percolating conductive network that results in higher capacity. In their work, $\mathrm{TiO}_{2}$ was in the form of nanorods synthesized by an expensive colloidal route that is not easily scalable, while in this work nanoparticles are used, and due to inter-particle necking that occurs as a result of sintering (Figure F.4), mesoporous monolith with optimum electrolyte contacting is built. While particle interconnectivity boosts performance, the sintering itself plays an additional role in attaining higher capacity. As we have reported elsewhere [18], increased crystallinity allows lithium to insert more easily into the octahedral sites of anatase due to favorable diffusion pathways increasing capacity. Here, the sintering step acts as a controlled anneal, increasing crystallinity and increasing diffusion-based Li-ion storage---the major insertion 

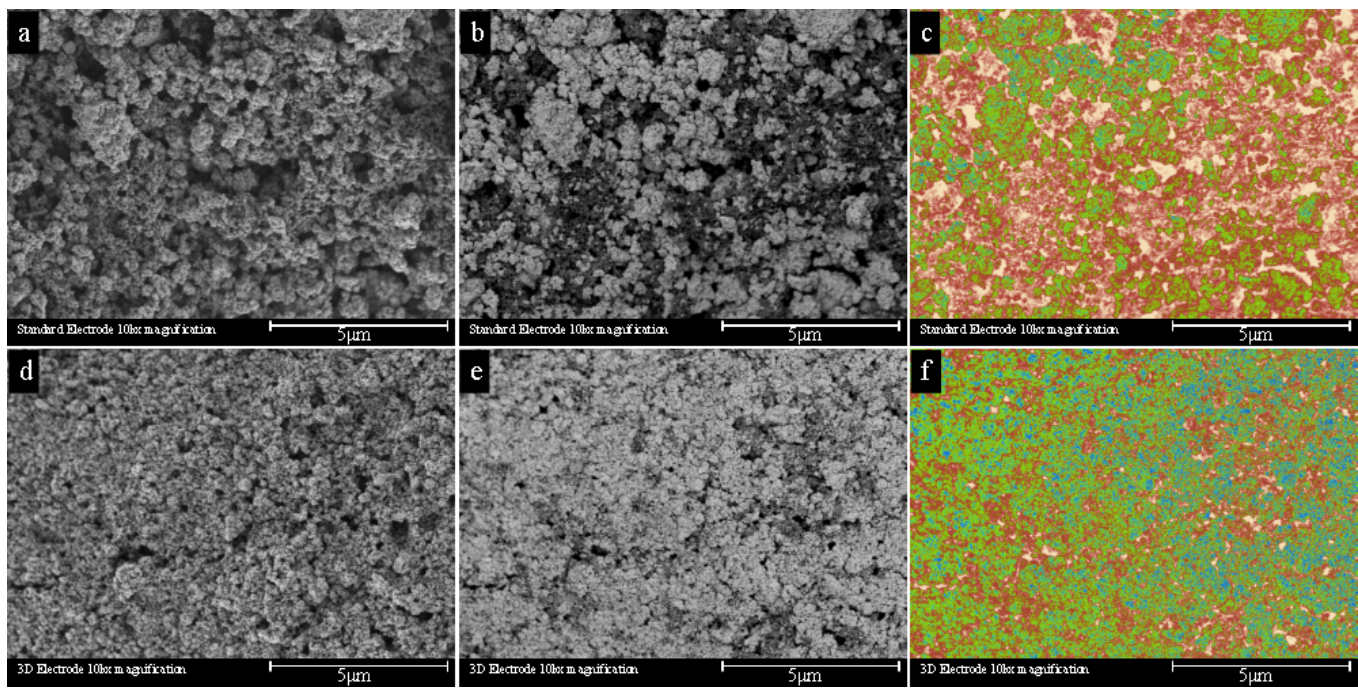

Figure 6.1: SEM micrographs showing topographical, composition, and compositional heat maps for standard (a-c) and 3-D (d-f) electrodes. Heat maps highlight electrode macropores (white), carbon (red), $\mathrm{TiO}_{2}$ (green), and mesopores within $\mathrm{TiO}_{2}$ aggregates (blue). 

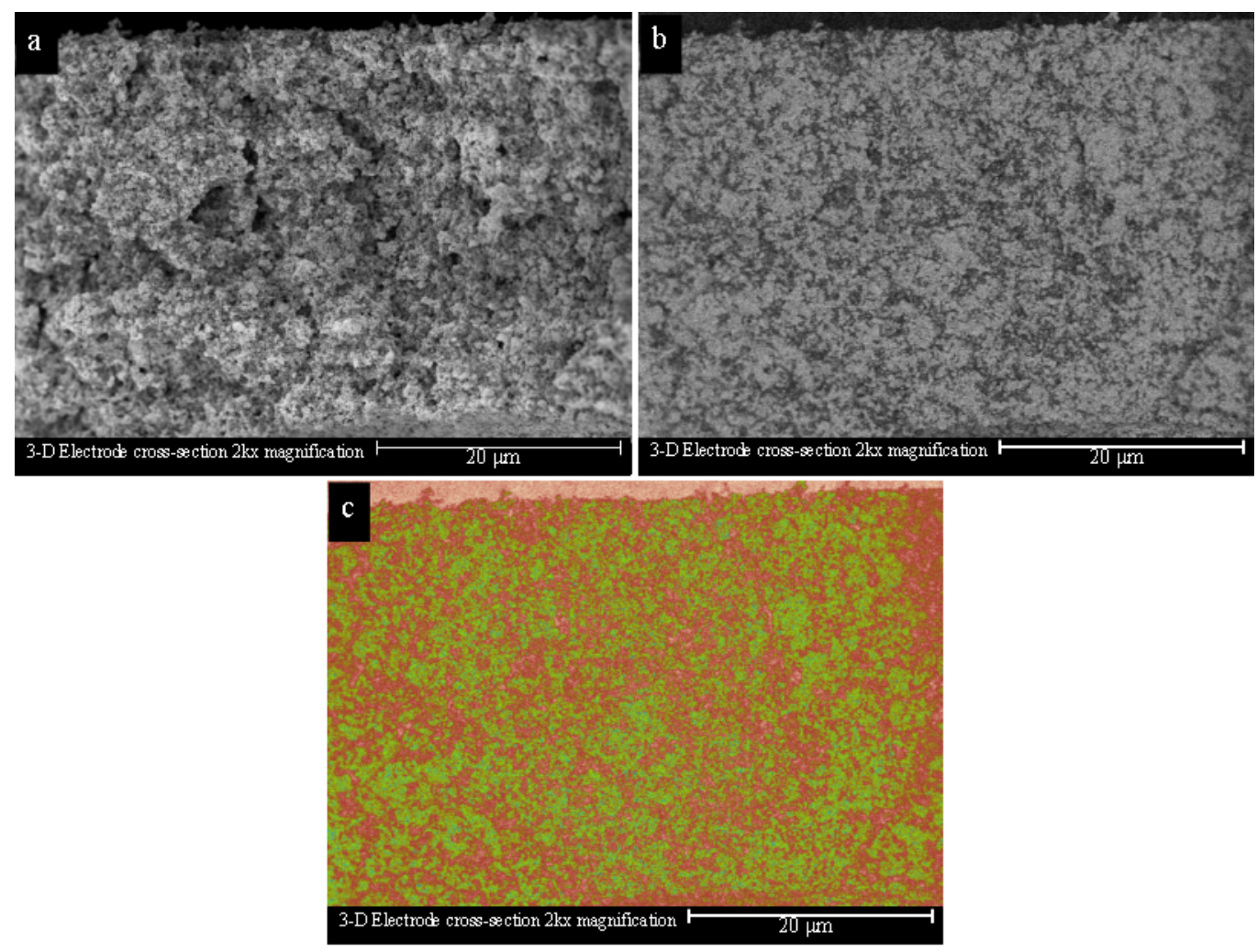

Figure 6.2: Cross-section of 3-D electrode film displaying percolating carbon networks as a topographic micrograph (a), compositional micrograph (b), and a heat map (c) that associates the two images. 


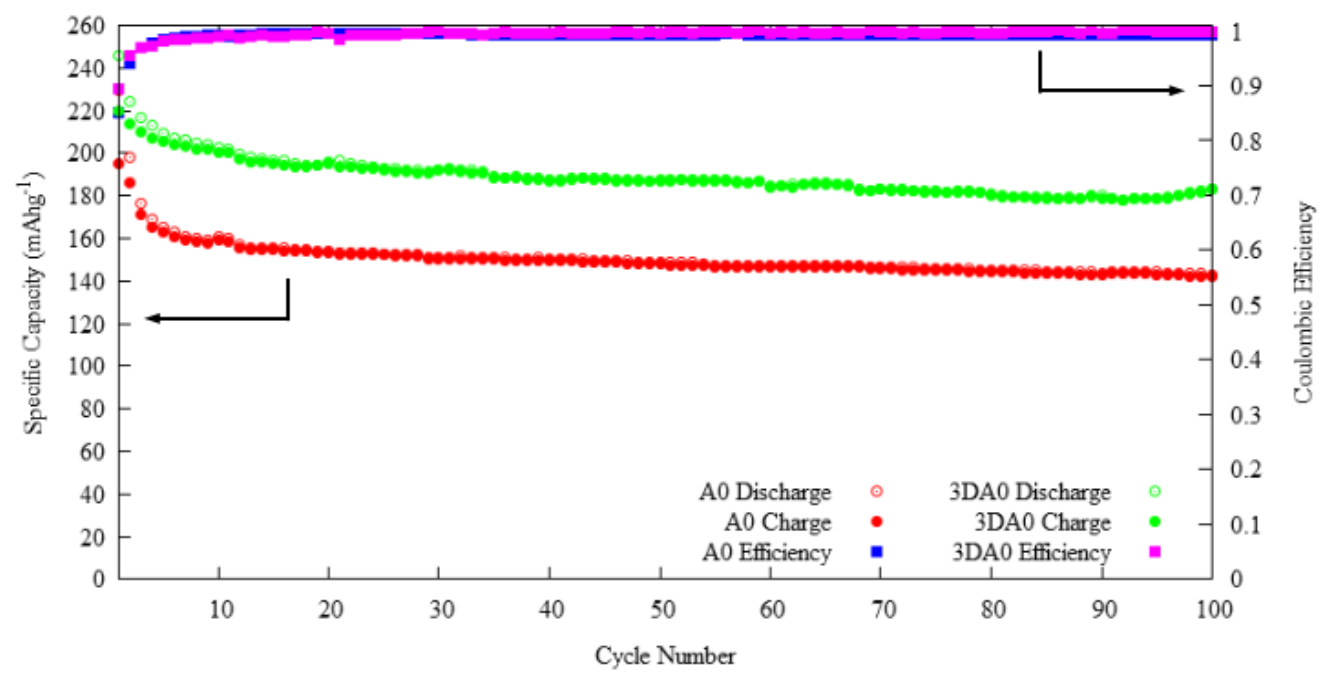

Figure 6.3: Galvanostatic charge/discharge over 100 cycles between cutoff voltages of $1 \mathrm{~V}$ (discharge) and $3 \mathrm{~V}$ (charge) at $1 \mathrm{C}$ rate $\left(168 \mathrm{~mA} \mathrm{~g}^{-1}\right)$

mechanism below $4 \mathrm{C}$ rate.

Further, the relative contribution of diffusion-based (intercalation) and pseudocapacitive storage depends on the degree of crystallinity and surface area of the anatase [18]. This intrinsic Li-ion host behavior of nanoanatase manifests when the rate capability of the 3-D electrodes is examined (Figure 6.4). At low rates (up to 5C), the 3-D electrode exhibits higher capacity than the standard electrode; thereafter the two electrodes have nearly the same capacity, e.g. $105 / 115 \mathrm{~mA} \mathrm{~h}^{-1}$ for the 3-D/standard electrodes, respectively, cycled at 10C rate for 100 cycles (Figure F.6). Due to sintering, the 3-D electrodes anatase particles have better crystallinity- a property that favors diffusion-based storage [18], resulting in higher capacity at low rate. Sintering also leads to reduction of surface area (from $222 \mathrm{~m}^{2} \mathrm{~g}^{-1}$ of the non-sintered crystallites to $100 \mathrm{~m}^{2} \mathrm{~g}^{-1}$ for the 3-D sintered film), causing significant loss of pseudocapacitive storage, hence the lower performance at higher rate.

It is important that sintering is carefully optimized to minimize surface area loss while maintaining the 3-D structure via inter-particle necking, crystal annealing, 
and mesopore formation via volatilization of the paste organic components. In the present system, optimal sintering is achieved with the thermal profile described in Figure F.7. The importance of optimizing sintering temperature and time is demonstrated with additional data (Figure F.8 and F.9) and discussion presented in the SI section.

We have demonstrated a novel electrode fabrication method using $6 \mathrm{~nm}$ aqueous synthesized anatase nanocrystals as active Li-ion host material. A controlled sintering procedure provided optimal 3-D structure without losing surface-area-based storage capability. These 3-D sintered electrodes have superior electrochemical properties. Further, the excellent intermixing of active particles and carbon achieved with the novel formulation and controlled sintering step leads to a highly conductive percolating carbon network. It is clear that this 3-D binder-free sintered electrode structure has potential to provide superior electrodes in a scalable and cost-effective way. While $\mathrm{TiO}_{2}$ was used as active material, it serves only as a platform to demonstrate the 3-D electrode engineering concept, which can be adapted for use with other active materials to produce cells with superior properties, meeting the everincreasing demand for cost-competitive advanced $\mathrm{Li}$-ion batteries by the electric vehicle industry. 


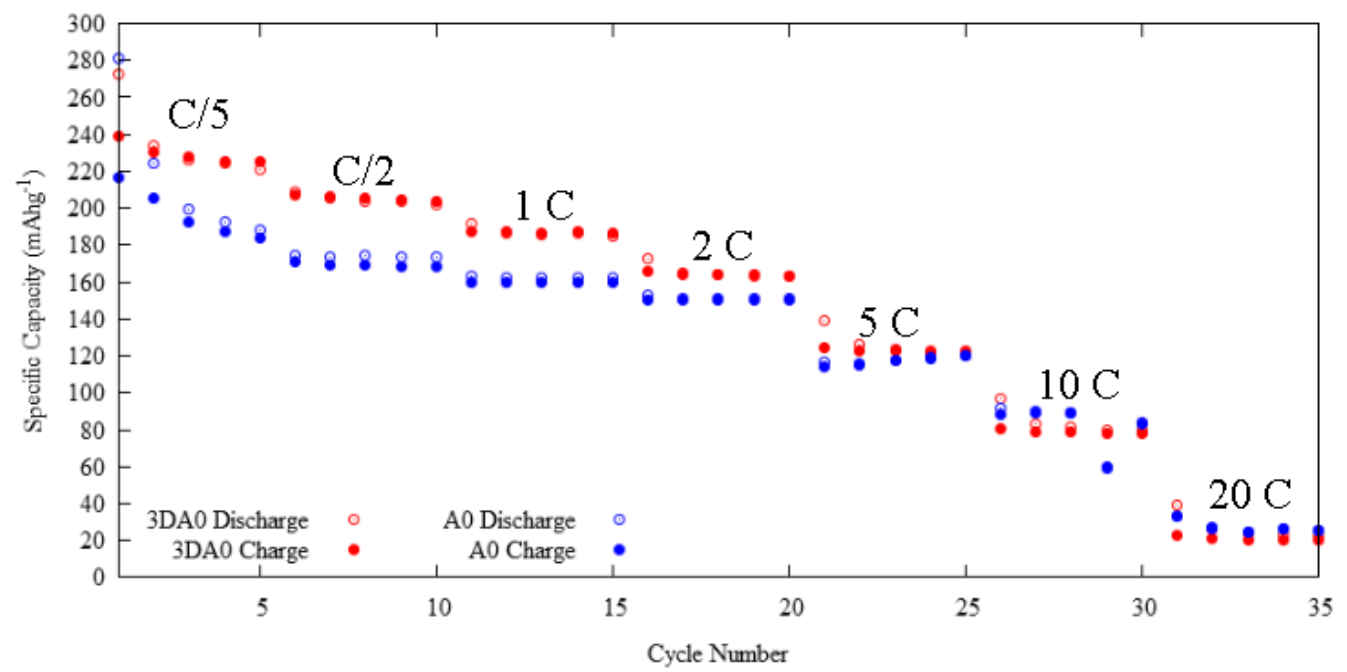

Figure 6.4: Galvanostatic charge/discharge over 100 cycles between cutoff voltages of $1 \mathrm{~V}$ (discharge) and $3 \mathrm{~V}$ (charge) at $1 \mathrm{C}$ rate $\left(168 \mathrm{~mA} \mathrm{~g}^{-1}\right)$

\subsection{Acknowledgements}

This research is supported by a strategic project grant from the Natural Sciences and Engineering Research Council of Canada (NSERC) and sponsored by Hydro-Quebec's Energy Conversion and Storage department. Amrita Yasin and Dr. Fuqiang Guo are thanked for their help with anatase synthesis.

\subsection{References}

[1] J. Li, C. Daniel, D. Wood, J. Power Sources, 196, 2452 (2011).

[2] J. Li, R. B. Lewis, J. R. Dahn, Electrochem. Solid State Lett., 10, A17 (2007).

[3] M. Mancini, F. Nobili, R. Tossici, M. Wohlfahrt-Mehrens, R. Marassi, J. Power Sources, 196, 9655 (2011).

[4] A. Morretti, G.-T. Kim, D. Bresser, K. Renger, E. Paillard, R. Marassi, M. Winter, S. Passerini, J. Power Sources, 221, 419 (2013). 
[5] J. Liu, Q. Zhang, Z.-Y. Wu, J.-H. Wu, J.-T. Li, L. Huang, S.-G. Sun, Chem. Comm., accepted (2014).

[6] Y.-H. Chen, C.-W. Wang, X. Zhang, A. M. Sastry, J. Power Sources, 195, 2851(2010).

[7] K.-T. Lee, J. C. Lytle, N. S. Ergangf, S. M. Oh, A. Stein, Adv. Funct. Mater, 15, 547 (2005).

[8] C. M. Doherty, R. A. Caruso, B. M. Smarsly, P. Adelhelm, C. J. Drummond, Chem. Mater, 21, 5300 (2009).

[9] D.-Y. Ha, M. A. Islam, R. D. Robinson, Nano Lett., 12, 5122 (2012).

[10] Z. Weng, H. Guo, X. Liu, S. Wu, K. W. K. Yeung, P. K. Chu, RSC Adv. 3, 24758 (2013).

[11] G. F. Ortiz, I. Hanzu, T. Djenizian, P. Lavela, J. L. Tirado, P. Knauth, Chem. Mater, 21, 63 (2009).

[12] T. Brezesinski, J. Wang, J. Polleux, B. Dunn, S. H. Tolbert, J. Am. Chem. Soc., 131, 1802 (2009).

[13] S. Lee, J. Ha, J. Choi, T. Song, J. W. Lee, U. Paik, ACS Appl. Mater. Interfaces, 5, 11525 (2013).

[14] P. Tammawat, N. Meethong, J. Nanomater., 2013, 1 (2013).

[15] J. Cheng, B. Wang, H. L. Xin, C. Kim, F. Nie, X. Li, G. Yang, H. Huang, J. Mater. Chem. A, 2, 2701 (2014).

[16] J. Chen, Recent Pat. Nanotech., 7, 2 (2013).

[17] M. J. Sussman, N. Brodusch, R. Gauvin, G. P. Demopoulos, J. Electrochem. Soc., 160, A3100 (2013).

[18] M. J. Sussman, M. Celikin, A. Yasin, G. P. Demopoulos, J. Power Sources Submitted (2014).

[19] D. Bresser, E. Paillard, E. Binetti, S. Krueger, M. Striccoli, M. Winter, S. Passerini, J. Power Sources, 206, 301 (2012). 
[20] K.-E. Lee, C. Charbonneau, G. Shan, G. P. Demopoulos, R. Gauvin, JOM, 61, 52 (2009). 



\section{Chapter 7}

\section{Synopsis}

In this chapter, global conclusions are drawn from the work reported in chapters

4-7. They refer to the binder-free fabrication of $\mathrm{TiO}_{2} / \mathrm{C}$ composite electrodes, characterization of mesoporous brookite nanoplatelets, electrochemical characterization of aqueous anatase nanoparticles, and the development of binder-free electrodes using aqueous anatase nanoparticles.

The conclusions are followed by claims to originality associated with the work presented in this thesis, and some suggested future work in the areas listed above.

\subsection{Global Conclusions}

The following major conclusions can be drawn from the work presented previously. They are presented in the order of the chapters of this thesis.

1. A binder-free fabrication method was developed that uses a chemo-mechanical process to homogeneously disperse active $\mathrm{TiO}_{2}$ and carbon into a paste that after doctor blading is sintered via controlled thermal profile to build a selfsupporting structure.

2. By removing the PVDF binder, and replacing with organic additives ( $\alpha$-terpineol and ethyl cellulose) that volatilize upon sintering, a mesoporous structure is developed with uniformly dispersed carbon exhibiting enhanced conductivity, leading to facile lithium insertion. Sintering the electrode causes interparticle necking which provides better interconnection between particles. Both of 
these lead to an increase in capacity, compared to the standard electrode, of $25 \%$.

Relevance: By removing binders, non-electroactive mass is eliminated from the electrode. The specific capacity of an active material is used to determine the mass required to achieve the desired capacity. However, in standard electrodes a percentage of the electrode mass is given to binders in order to provide structure. By removing this, more active materials loading is possible per unit mass. As well, by increasing capacity, less material is required to achieve the same capacity. Finally, by dispersing the components homogeneously, a more conductive film with smaller aggregates is formed providing better properties without requiring carbon coating of particles or other costly conductivity enhancing techniques.

3. New variety of aqueous synthesized brookite $\mathrm{TiO}_{2}$ having mesoporous nanoplatelet morphology was electrochemically evaluated as a Li-ion host material, and its post-cycling crystal structure was probed. This platelet shape led to a stable specific capacity of $\sim 185 \mathrm{~mA} \mathrm{~h} \mathrm{~g}^{-1}$ at $\mathrm{C} / 10$ rate after 50 cycles, and excellent capacity retention at both low and high rate.

4. Using a combination of electrochemical impedance spectroscopy and X-ray diffraction techniques, the lithiation mechanism was investigated. In-situ EIS was employed during the lithiation in the formation step, as well as XRD after complete lithiation and delithiation, determined that a solid solution of $\mathrm{Li}$ and $\mathrm{TiO}_{2}$ was formed. The resulting $\mathrm{Li}_{\mathrm{x}} \mathrm{TiO}_{2}$ retained the crystal structure of pristine brookite, expanding the crystal slightly to allow for lithium to fit in the interstitial sites.

Relevance: Until now, the synthesis of brookite was only possible using highly acidic or basic environments, or by decomposing complex intermediates. These have also been spherical nanoparticles, while the aqueous synthesized brookite is a 
2-D morphology that can be synthesized in large quantity with a simple stirred-tank reactor. While brookite requires a slow cycling time to achieve high capacity as compared to other phases of $\mathrm{TiO}_{2}$, its morphology provides unparalleled stability, as compared to other phases and previously synthesized brookite morphologies. The effects of the nanoplatelet morphology can also be extended to other phases of $\mathrm{TiO}_{2}$, or other materials, to enhance their intrinsic electrochemical properties. Most importantly, by understanding the effects of lithiation on the crystal structure, the crystal can be manipulated so that the lithium can more easily, and quickly, enter the crystal. This would enhance rate capability, making brookite a more viable active material.

5. Novel aqueous synthesized $6 \mathrm{~nm}$ anatase nanoparticles were annealed at various temperatures to determine the effects of crystallinity and surface area on Li-ion storage properties by differentiating between diffusion-based (intercalation) and surface-area-based (pseudocapacitive) modes.

6. Annealing to increase crystallinity results in increased Li-ion diffusivity within the anatase crystal, increasing the capacity at low rate $(\leq 4 C)$. While annealing causes particle coarsening and loss of surface area, annealing at $300{ }^{\circ} \mathrm{C}$ increases the crystallinity enough that diffusion-based storage is still facile at high rate $(10 \mathrm{C})$, resulting in a reversible capacity that is comparable to that of as-synthesized anatase. However, as the rate increases, the increased crystallinity and decreased surface area causes increased polarization, which manifests in long-term (500+ cycles) stability loss due to apparent electrolyte reactivity and breakdown.

Relevance: The aqueous synthesis of anatase nanoparticles provides an opportunity to expand $\mathrm{TiO}_{2}$ into the realm of commercially viable Li-ion active materials. Understanding the relationship between annealing-induced crystallinity and surface area makes it easier to tailor the physical properties of nanoparticles in order to 
achieve the desired electrochemical characteristics. The end result is that a balance between surface area and crystallinity provides the optimal conditions for lithiation.

7. 3-Dimensional electrodes engineered using the binder-free fabrication method with the unannealed aqueous synthesized anatase nanoparticles were characterized physically and electrochemically. Further confirmation of the percolating carbon networks, and understanding of the importance of controlled sintering was obtained.

8. At $1 \mathrm{C}$ rate, there was a significant increase in capacity over 100 cycles between the industry standard and 3-D electrodes. The industry standard electrode achieved $150 \mathrm{~mA} \mathrm{~h} \mathrm{~g}^{-1}$ while the 3-D electrode achieved $180 \mathrm{~mA} \mathrm{~h} \mathrm{~g}^{-1}$, both with a Coulombic efficiency of $\sim 100 \%$. At high rate (10C) the 3-D electrode had slightly lower capacity compared to the standard electrode (105 $\mathrm{mA} \mathrm{h} \mathrm{g}^{-1}$ compared to $115 \mathrm{~mA} \mathrm{~h} \mathrm{~g}^{-1}$, respectively), but showed higher capacity retention.

Relevance: While it was known (from work seen in previous chapters) that using the binder-free fabrication process, and annealing provides significant increases to the capacity of anatase electrodes at low rate (1C), the full effect of the process was not understood. By controlled sintering, high temperature at short times provides annealing with minor coarsening, giving the low rate benefit seen with the anatase nanopowder annealed at $300^{\circ} \mathrm{C}$. However, the added benefit of dispersants and inter-particle necking provides better conductive networks (both ionic and electronic), allowing for increased capacity at high rate ( $\geq 5 C$ rate) as compared to pre-annealed nanopowders. 


\subsection{Claims to Originality}

1. It is the first time that a nanaotitania/carbon paste has been formulated, which upon controlled sintering following doctor blading (or screen printing), is demonstrated as binder-free fabrication process for Li-ion electrodes. While other self-supporting electrode fabrication processes exist, never before has a method been developed that could take commercially available active and conductive components and build electrodes with significant increases in capacity.

2. For the first time, a quantitative analysis technique using scanning electron microscopy and image analysis was successfully applied to quantitatively characterize the distribution of conductive additives, and therefore active material within a Li-ion electrode.

3. It is the first time that aqueous synthesized mesoporous brookite nanoplatelets have been characterized electrochemically. All previous morphologies of brookite $\mathrm{TiO}_{2}$ were nanoparticles, thus no electrochemical data existed for 2-D nanoplatelets.

4. For the first time, the effect of multi-cycle lithium insertion and extraction on the relaxation of the crystal structure of brookite was successfully examined, experimentally confirming previously reported theoretical simulations. In addition, it was discovered upon repeated cycling the structure of brookite nanoplatelets to undergo crystal refinement resulting in highly stable reversible electrode performance.

5. For the first time, aqueous synthesized anatase nanocrystallites $(6 \mathrm{~nm}$ diameter) were electrochemically characterized in terms of their Li-ion storage properties distinguishing between pseudocapacitive and intercalation modes. 
6. While surface area has been investigated in its role in pseudocapacitive storage, it has never been compared to diffusion-based intercalation (found to depend on degree of crystallinity) to determine the point where it becomes the main mechanism for lithium-ion storage in anatase.

7. This is the first time that aqueous anatase has been fabricated into electrodes using the binder-free fabrication process to produce 3-D monolithic electrodes. This method had only been tested on commercially available nanoparticles previously.

8. Finally, it is the first time that the importance of annealing time and temperature control has been investigated.

\subsection{Future Work}

The following recommendations on potential further investigation is presented, relating to aqueous synthesized brookite and anatase, as well as 3-D electrodes assembled with aqueous anatase particles using the binder-free fabrication method:

- While the effect of lithiation on the crystal structure of brookite was investigated, an ex-situ XRD was required due to the current lack of in-situ test set-up. It is recommended that the in-situ XRD to understand the real-time changes in crystal structure during lithiation be performed. This, coupled with XPS to determine if surface storage plays a significant part in lithiation, would provide a global understanding of the mechanisms involved in the lithiation of brookite.

- Annealing of aqueous synthesized anatase was performed at various temperatures for 24 hours. This led to significant loss of surface area. A further study of annealing for various lengths of time at $300^{\circ} \mathrm{C}$ as well as $400{ }^{\circ} \mathrm{C}$ 
and $450^{\circ} \mathrm{C}$ to increase crystallinity and limit loss of surface area, should be performed in order to optimize synthesis product for use in electrodes.

- Fabrication of 3-D electrodes using spacers with lower burn-out temperature, while experimenting with annealing/necking temperatures lower than $450{ }^{\circ} \mathrm{C}$ could be pursued as an avenue to further processing simplification. As well, experimentation with multi-layered electrode films to add active mass to each electrode without having to contend with increased substrate mass would be a logical next step.

Finally, a parallel direction should be taken to attempt to carbon-coat the aqueous anatase nanocrystallites. Once coated successfully, it should be characterized using galvanostatic, potentiostatic, and EIS techniques. The coated anatase should be built into 3-D electrodes potentially eliminating altogether the use of conductive additives without sacrificing Li-ion storage capacity. 



\section{Appendix A}

\section{X-ray Diffraction Peak List}

On the following pages are the diffraction pattern peak lists for anatase, rutile, brookite, and $\mathrm{Li}_{0.5} \mathrm{TiO}_{2}$. Listed are the $h k l$ values for the planes associated with the peaks, the d-spacings and the $2 \theta$ values. These values were used to determine phases and to aid in mathematical refinement using the Rietveld method. The peak

locations and relative intensities are shown graphically in the experimental section of this thesis. 
Table A.1: JCPDS crystal phase parameters of anatase (\#00-021-1272)

\begin{tabular}{ccccc}
\hline $\mathrm{h}$ & $\mathrm{k}$ & 1 & $\mathrm{~d}(\AA)$ & $2 \theta$ \\
\hline 1 & 0 & 1 & 3.520 & 25.281 \\
1 & 0 & 3 & 2.431 & 36.947 \\
0 & 0 & 4 & 2.378 & 37.801 \\
1 & 1 & 2 & 2.332 & 38.576 \\
2 & 0 & 0 & 1.892 & 48.050 \\
1 & 0 & 5 & 1.699 & 53.891 \\
2 & 1 & 1 & 1.667 & 55.062 \\
2 & 1 & 3 & 1.493 & 62.121 \\
2 & 0 & 4 & 1.481 & 62.090 \\
1 & 1 & 6 & 1.364 & 68.762 \\
2 & 2 & 0 & 1.338 & 70.311 \\
1 & 0 & 7 & 1.279 & 74.031 \\
2 & 1 & 5 & 1.265 & 75.032 \\
3 & 0 & 1 & 1.251 & 76.020 \\
0 & 0 & 8 & 1.189 & 80.727 \\
3 & 0 & 3 & 1.172 & 82.139 \\
2 & 2 & 4 & 1.166 & 82.662 \\
3 & 1 & 2 & 1.160 & 83.149 \\
2 & 1 & 7 & 1.060 & 93.221 \\
3 & 0 & 5 & 1.051 & 94.182 \\
3 & 2 & 1 & 1.043 & 95.143 \\
1 & 0 & 9 & 1.018 & 98.319 \\
2 & 0 & 8 & 1.007 & 99.804 \\
3 & 2 & 3 & 0.996 & 101.221 \\
3 & 1 & 6 & 0.955 & 107.448 \\
4 & 0 & 0 & 0.946 & 108.963 \\
3 & 0 & 7 & 0.924 & 112.841 \\
3 & 2 & 5 & 0.919 & 113.861 \\
4 & 1 & 1 & 0.913 & 114.909 \\
2 & 1 & 9 & 0.896 & 118.439 \\
2 & 2 & 8 & 0.889 & 120.104 \\
4 & 1 & 3 & 0.881 & 121.725 \\
4 & 0 & 4 & 0.879 & 122.336 \\
4 & 2 & 0 & 0.846 & 131.036 \\
3 & 2 & 7 & 0.830 & 135.998 \\
4 & 1 & 5 & 0.826 & 137.391 \\
3 & 0 & 9 & 0.810 & 143.888 \\
4 & 2 & 4 & 0.797 & 150.039 \\
0 & 0 & 12 & 0.792 & 152.634 \\
\hline & & & &
\end{tabular}


Table A.2: JCPDS crystal phase parameters of rutile (\#00-021-1276)

\begin{tabular}{ccccc}
\hline $\mathrm{h}$ & $\mathrm{k}$ & 1 & $\mathrm{~d}(\AA)$ & $2 \theta$ \\
\hline 1 & 1 & 0 & 3.247 & 27.447 \\
1 & 0 & 1 & 2.487 & 36.086 \\
2 & 0 & 0 & 2.297 & 39.188 \\
1 & 1 & 1 & 2.188 & 41.226 \\
2 & 1 & 0 & 2.054 & 44.052 \\
2 & 1 & 1 & 1.687 & 54.323 \\
2 & 2 & 0 & 1.623 & 56.642 \\
0 & 0 & 2 & 1.479 & 62.742 \\
3 & 1 & 0 & 1.452 & 64.040 \\
2 & 2 & 1 & 1.424 & 65.480 \\
3 & 0 & 1 & 1.359 & 69.010 \\
1 & 1 & 2 & 1.346 & 69.790 \\
3 & 1 & 1 & 1.304 & 72.410 \\
3 & 2 & 0 & 1.273 & 74.411 \\
2 & 0 & 2 & 1.244 & 76.510 \\
2 & 1 & 2 & 1.200 & 79.822 \\
3 & 2 & 1 & 1.170 & 82.335 \\
4 & 0 & 0 & 1.148 & 84.260 \\
4 & 1 & 0 & 1.114 & 87.464 \\
2 & 2 & 2 & 1.093 & 89.557 \\
3 & 3 & 0 & 1.082 & 90.708 \\
4 & 1 & 1 & 1.042 & 95.275 \\
3 & 1 & 2 & 1.036 & 96.017 \\
4 & 2 & 0 & 1.027 & 97.177 \\
3 & 3 & 1 & 1.016 & 98.514 \\
4 & 2 & 1 & 0.970 & 105.099 \\
1 & 0 & 3 & 0.964 & 106.019 \\
1 & 1 & 3 & 0.943 & 109.406 \\
4 & 0 & 2 & 0.907 & 116.227 \\
5 & 1 & 0 & 0.900 & 117.527 \\
2 & 1 & 3 & 0.889 & 120.059 \\
4 & 3 & 1 & 0.877 & 122.788 \\
3 & 3 & 2 & 0.873 & 123.660 \\
4 & 2 & 2 & 0.843 & 131.847 \\
3 & 0 & 3 & 0.829 & 136.549 \\
5 & 2 & 1 & 0.819 & 140.052 \\
4 & 4 & 0 & 0.812 & 143.116 \\
5 & 3 & 0 & 0.787 & 155.870 \\
\hline & & & &
\end{tabular}


Table A.3: JCPDS crystal phase parameters of brookite (\#00-016-0617)

\begin{tabular}{ccccc}
\hline $\mathrm{h}$ & $\mathrm{k}$ & 1 & $\mathrm{~d}(\AA)$ & $2 \theta$ \\
\hline 1 & 2 & 0 & 3.512 & 25.340 \\
1 & 1 & 1 & 3.465 & 25.689 \\
1 & 2 & 1 & 2.900 & 30.808 \\
2 & 0 & 0 & 2.729 & 32.791 \\
0 & 1 & 2 & 2.476 & 36.252 \\
2 & 0 & 1 & 2.406 & 37.297 \\
1 & 3 & 1 & 2.370 & 37.934 \\
2 & 2 & 0 & 2.344 & 38.371 \\
2 & 1 & 1 & 2.332 & 38.576 \\
0 & 4 & 0 & 2.296 & 39.205 \\
1 & 1 & 2 & 2.254 & 39.967 \\
0 & 2 & 2 & 2.244 & 40.153 \\
2 & 2 & 1 & 2.133 & 42.340 \\
0 & 3 & 2 & 1.636 & 46.060 \\
2 & 3 & 1 & 1.896 & 48.023 \\
1 & 3 & 2 & 1.851 & 49.184 \\
2 & 1 & 2 & 1.833 & 49.699 \\
2 & 4 & 0 & 1.757 & 52.006 \\
3 & 2 & 0 & 1.691 & 54.198 \\
2 & 4 & 1 & 1.662 & 55.223 \\
1 & 5 & 1 & 1.649 & 55.697 \\
1 & 1 & 3 & 1.610 & 57.168 \\
2 & 3 & 2 & 1.597 & 57.677 \\
1 & 2 & 3 & 1.541 & 59.983 \\
0 & 5 & 2 & 1.494 & 62.075 \\
1 & 6 & 0 & 1.473 & 63.060 \\
3 & 1 & 2 & 1.466 & 63.396 \\
2 & 5 & 1 & 1.461 & 63.639 \\
2 & 0 & 3 & 1.452 & 64.080 \\
1 & 3 & 3 & 1.442 & 64.578 \\
2 & 1 & 3 & 1.434 & 64.982 \\
1 & 6 & 1 & 1.417 & 65.860 \\
4 & 0 & 0 & 1.364 & 68.768 \\
3 & 3 & 2 & 1.336 & 70.420 \\
4 & 0 & 1 & 1.319 & 71.465 \\
2 & 3 & 3 & 1.312 & 71.906 \\
0 & 0 & 4 & 1.285 & 73.662 \\
0 & 2 & 4 & 1.238 & 76.956 \\
4 & 3 & 1 & 1.211 & 79.001 \\
\hline & & & &
\end{tabular}


Table A.4: JCPDS crystal phase parameters of $\mathrm{Li}_{0.5} \mathrm{TiO}_{2}(\# 00-038-0269)$

\begin{tabular}{ccccc}
\hline $\mathrm{h}$ & $\mathrm{k}$ & 1 & $\mathrm{~d}(\AA)$ & $2 \theta$ \\
\hline 1 & 0 & 1 & 3.720 & 23.901 \\
0 & 1 & 1 & 3.524 & 25.252 \\
1 & 0 & 3 & 2.421 & 37.105 \\
1 & 1 & 2 & 2.372 & 37.901 \\
0 & 0 & 4 & 2.257 & 39.911 \\
2 & 0 & 0 & 2.037 & 44.439 \\
0 & 2 & 0 & 1.909 & 47.596 \\
2 & 1 & 1 & 1.763 & 51.816 \\
1 & 2 & 1 & 1.697 & 53.991 \\
0 & 1 & 5 & 1.630 & 56.403 \\
2 & 0 & 4 & 1.511 & 61.300 \\
0 & 2 & 4 & 1.458 & 63.785 \\
2 & 2 & 0 & 1.392 & 67.198 \\
2 & 1 & 5 & 1.274 & 74.405 \\
1 & 2 & 5 & 1.246 & 76.372 \\
2 & 2 & 4 & 1.185 & 81.09 \\
\hline
\end{tabular}





\section{Appendix B}

\section{Experimental Methods}

\section{B.1 Introduction}

In this chapter, all experimental details relating to the chemicals, materials, laboratory equipment, analytical equipment, procedures and methods used are provided. The first section describes all of the chemicals and materials used in the synthesis of active material, fabrication of electrodes, and construction of electrochemical cells. In the second section, active material synthesis, substrate treatment, electrode fabrication, and cell assembly methods are described. Finally, the analytical tools employed in the characterization of the active materials and electrode films are provided and for each characterization method, the sample preparation and operating conditions are explained.

\section{B.2 Chemicals and Materials}

\section{B.2.1 Synthesis of Active Materials}

The synthesis of the aqueous nanotitania involved forced hydrolysis of an aqueous $\mathrm{TiCl}_{4}$ solution of variable concentrations to produce various phases of titania. The reaction controlling this forced hydrolysis reaction is

$$
\mathrm{TiCl}_{4(\mathrm{l})}+2 \mathrm{H}_{2} \mathrm{O}_{(1 / \mathrm{g})} \longrightarrow \mathrm{TiO}_{2(\mathrm{~s})}+4 \mathrm{HCl}+\Delta H
$$


Synthesis requires titanium tetrachloride $\left(\mathrm{TiCl}_{4}\right.$, Sigma Aldrich), previously diluted to a $2 \mathrm{M}$ solution, as well as ammonium hydroxide (29\% assay, Fisher Scientific). Synthesis was carried out in a continuous stirred-tank reactor as per the method developed in our laboratory [1], following initial investigations by Charbonneau, et al. [2,3]. A schematic of the synthesis process can be seen in Figure B.2. In a typical synthesis run for brookite a $0.1 \mathrm{M} \mathrm{TiCl}_{4(\text { (aq) }}$ solution was continuously pumped into a stirred reactor preheated to $80^{\circ} \mathrm{C}$. After the attainment of steady-state operation at 1 hour mean residence time a colloidal nanobrookite suspension was produced. The outflow was collected and treated with $\mathrm{NH}_{4} \mathrm{OH}$ to $\mathrm{pH}=3$ to promote colloid aggregation, an important step in its recovery via centrifugation [1]. The material was washed 3 times with DI water and once with anhydrous ethanol, then dried at $80^{\circ} \mathrm{C}$. For anatase, the $\mathrm{pH}$ is controlled within the reactor to $\mathrm{pH}=3$, rather than treated after outflow.
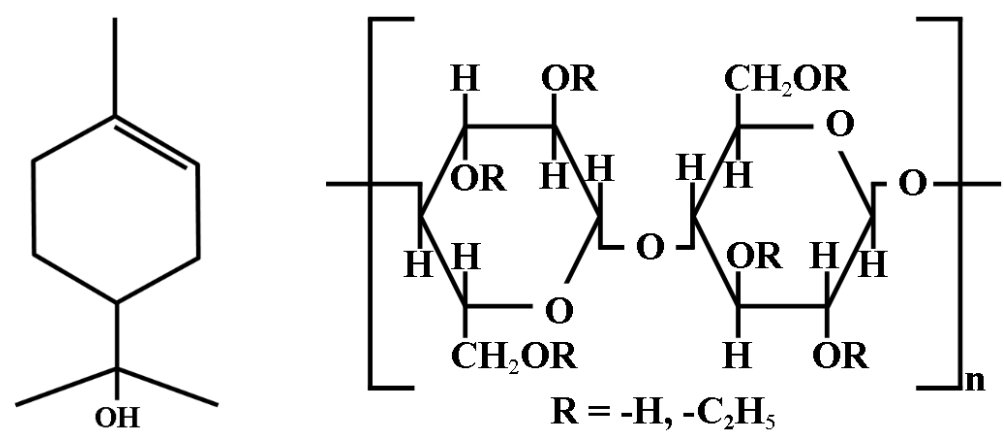

Figure B.1: Chemical structures of $\alpha$-terpineol (left) and ethyl cellulose (right). 


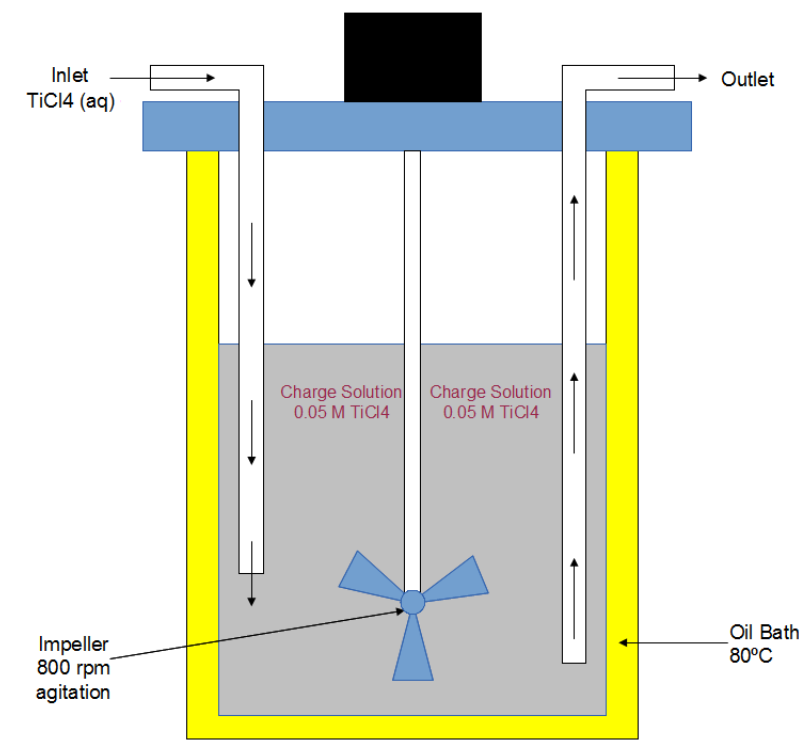

Figure B.2: Schematic of the continuous synthesis process.

\section{B.2.2 Electrode Fabrication}

The preparation of the electrodes involved the use of various powders, organic solvents, and reagents. Powders used were aqueous synthesized nanotitania phases as well as commerically available titania, namely P25 (Evonik, 80\% anatase, 20\% rutile, $\sim 30 \mathrm{~nm}$ ) [4], anatase (Alfa Aesar, $\sim 15 \mathrm{~nm}$, acetylene black (Sigma Aldrich), ethyl cellulose (ethoxy content 48\%, $100 \mathrm{cps}$, Acros Organics), poly(vinylidene) fluoride (PVDF 6020, Solvay), and oxalic acid crystals ( $\geq 99 \%$, Sigma Aldrich). Solvents and reagents include anhydrous ethanol (Fisher Scientific), acetic acid (glacial, 99.7\% assay, ACS reagent, Fisher Scientific), $\alpha$-terpineol (97+\%, Acros Organics), and n-methyl 2-pyrrolidone (NMP, $\geq 99 \%$ spectrophotometric grade, Sigma Aldrich).

FTO-coated conductive glass (Fluorine-doped Tin Oxide-coated glass, $7 \Omega \mathrm{cm}^{-2}$, Sigma Aldrich), and high purity aluminum foil (15 $\mu \mathrm{m}$ thickness, MTI Corp) were used as substrate for the preparation of the working electrode. Pre- and post- 
treatment of the glass substrates used $\mathrm{TiCl}_{4}$ aqueous solution $\left(99 \% \mathrm{TiCl}_{4}\right.$, Fisher Scientific), while a $10 \%$ solution of sodium hydroxide (99.3\%, Fisher Scientific) was used with the aluminum foil.

\section{B.2.3 Electrochemical Cell Assembly}

Two types of electrochemical cells were used. Three-electrode cells were used for cyclic voltammetry and initial galvanostatic charge/discharge testing, while two electrode Swagelok-type cells were used for cycle life testing and rate capability. Three electrode cells used lithium metal as the reference and counter electrode. Swagelok-type cells used lithium metal as the counter electrode. Electrolyte was $\mathrm{LiPF}_{6}$ salt dissolved in ethylene carbonate (EC), dimethyl carbonate (DMC), and diethyl carbonate (DEC) at a ratio of 2:2:1 by weight (Selectilyte Sample Series A2, BASF Group). Separators used were porous polypropylene sheet (MTI Corp).

\section{B.3 Treatment and Fabrication}

\section{B.3.1 Pre-treatment of Electrode Substrates}

FTO-glass and aluminum foils were used as substrates for the electrodes characterized throughout this work. FTO glass was cut into $2 \times 4.5 \mathrm{~cm}$ rectangles. FTO glass used with the binder-free fabrication method was first cleaned using ethanol and then UV-Ozone treated for 30 minutes. The glass was then chemically pretreated in $50 \mathrm{mM} \mathrm{TiCl}_{4}$ aqueous solution at $80{ }^{\circ} \mathrm{C}$ for 30 minutes. After treatment, the FTO glass was washed with deionized water and ethanol. The aluminum foil was cleaned with ethanol and chemically pre-treated in $10 \% \mathrm{NaOH}$ solution for 10 seconds to promote paste adhesion via etching of the aluminum oxide surface layer. 


\section{B.3.2 Electrode Fabrication}

\section{B.3.2.1 Binder-based Electrodes}

Binder based electrodes were fabricated by using a mechanical mixing method. First, a $10 \mathrm{wt} \%$ solution of PVDF in NMP was made. Then the active material and conductive additive (carbon black) were mixed and ground with a glass mortar and pestle, until a visibly homogeneous powder mixture remained. This mixture was transfered to a beaker, and the PVDF-NMP solution was added. The mixture was mixed using a stand mixer at $700 \mathrm{rpm}$ for 30 minutes, adding extra NMP to achieve the desired slurry viscosity.

The resulting slurry was then doctor bladed onto untreated FTO glass and aluminum foil. The electrodes were then dried in a vacuum oven for 24 hours at $95{ }^{\circ} \mathrm{C}$ at $-100 \mathrm{kPa}$ vacuum. The electrodes on FTO glass were put immediately into an environmentally controlled glovebox. The electrodes on aluminum foil were pressed (in the case of electrodes using brookite active material) at $590 \mathrm{MPa}$ for 5 minutes, then returned to the vacuum oven overnight at $120^{\circ} \mathrm{C} .15 \mathrm{~mm}$ diameter electrode discs were punched from the electrode film deposited on aluminum foil. The electrode discs were put into the vacuum oven overnight at $100{ }^{\circ} \mathrm{C}$ to remove moisture then transferred to the glovebox.

\section{B.3.2.2 Binder-Free Electrodes}

The fabrication method of the binder-free electrodes was based on a previous work by Ito, et al [5]. $6 \mathrm{~g}$ of titania active material was mixed with $0.5 \mathrm{~g}$ of acetylene black (conductive additive). The powders were ground using a glass mortar and pestle until the resulting mixture was a uniform grey color. $1 \mathrm{~mL}$ of acetic acid was added to the powder mixture and ground. $20 \mathrm{~mL}$ of anhydrous ethanol are added to the mortar $1.5 \mathrm{~mL}$ at a time, grinding after each addition. After the addition of the $20 \mathrm{~mL}$ of anhydrous ethanol, another $15 \mathrm{~mL}$ was added alternation $2.5 \mathrm{~mL}$ and 
grinding. The mixture was ground until no aggregates were visible, then $100 \mathrm{~mL}$ of anhydrous ethanol was added in order to dilute the colloidal suspension further. The suspension was transferred from the mortar to a beaker and mixed with a magnetic stirrer for 1 minute, then ultrasonicated using an ultrasonic horn in pulse mode for 2 seconds work and 2 seconds rest, then stirred again for 1 minute. $20 \mathrm{~g}$ of $\alpha$-terpineol was added, and the suspension was stirred, utrasonicated, and stirred. $3 \mathrm{~g}$ ethyl cellulose was added slowly to the suspension as it stirred. The suspension underwent three more stir/ultrasonication/stir cycles before being transferred to the rotary evaporator. The suspension was condensed to paste at $45^{\circ} \mathrm{C}$, removing $95 \mathrm{~mL}$ of ethanol.

The resulting paste was doctor bladed on to treated FTO glass or aluminum foil. The electrodes were then sintered in a controlled sintering process seen in Figure B.3. The sintering process involved a stepwise heating in order to decompose/volatilize organics and promote necking. The first temperature plateau occurred at $150^{\circ} \mathrm{C}$ for 15 minutes after heating at $120^{\circ} \mathrm{Ch}^{-1}$ from room temperature to $150^{\circ} \mathrm{C}$. After the first plateau, the temperature rises at $240^{\circ} \mathrm{Ch}^{-1}$ to $325^{\circ} \mathrm{C}$ for 5 minutes. Then the temperature increases at $120^{\circ} \mathrm{Ch}^{-1}$ to $375^{\circ} \mathrm{C}$ and holds for 5 minutes. The last plateau occurs at $450{ }^{\circ} \mathrm{C}$ after a $60^{\circ} \mathrm{Ch}^{-1}$ temperature increase. Finally, cooldown occurs at a rate of $60^{\circ} \mathrm{Ch}^{-1}$ to room temperature. Annealing was performed in a programmable furnace in air.

After sintering, electrodes deposited on FTO glass were post-treated in $50 \mathrm{mM}$ $\mathrm{TiCl}_{4}$ aqueous solution at $80^{\circ} \mathrm{C}$ for 30 minutes to achieve chemical bonding. The film was then lightly rinsed with deionized water and ethanol and annealed using the same temperature profile as was used in sintering. Once annealing was completed the electrodes were put in the vacuum oven over night to remove moisture from the porous structures, then put into the Ar-atmosphere controlled glovebox. 


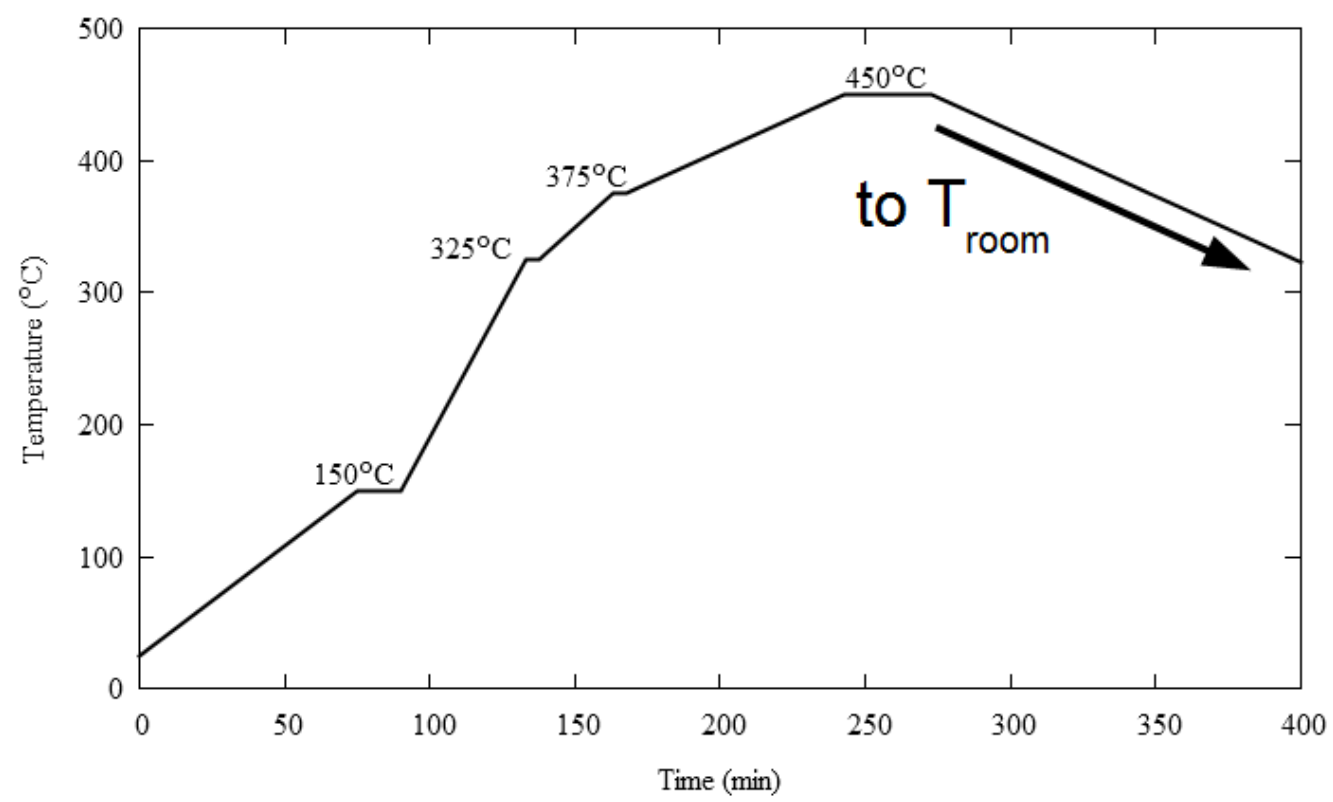

Figure B.3: Time/Temperature profile for controlled sintering process [6]

\section{B.3.3 Electrochemical Cell Fabrication}

All cell construction occurred within the confines of an argon filled glovebox. Two different three electrode cells were fabricated for this research, a commercial cell and an in-house cell. The commercial cell (Hohsen Corp., Osaka, Japan) utilized electrodes with aluminum foil substrate while the in-house cell used aluminum sheet or FTO glass substrate electrodes. For the commercial cell, the working electrode was the aluminum foil substrate electrode discs, which was fitted as seen in Figure B.4. A $24 \mathrm{~mm}$ electrolyte ( $\mathrm{LiPF}_{6}$ salt in EC:DMC:DEC) soaked separator was then placed on top of it. The reference electrode was a lithium metal disc with inner and outer diameters of 16 and $24 \mathrm{~mm}$, respectively. Another soaked separator was added, then a $16 \mathrm{~mm}$ lithium metal disc acting as the counter electrode. The cell was sealed tightly with bolts and removed from the glovebox. The cell was allowed to reach equilibrium for 4 hours before testing. 


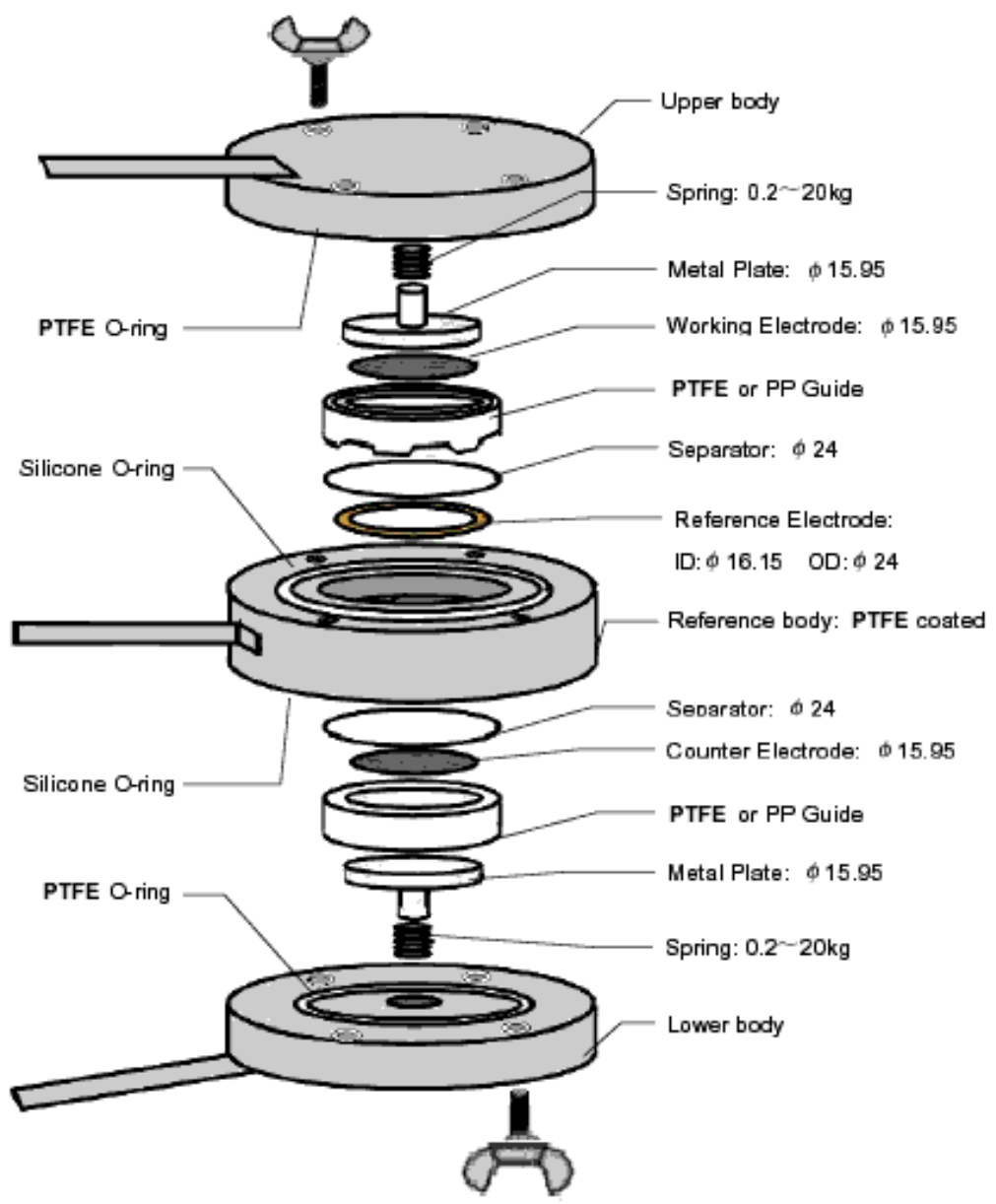

Figure B.4: Schematic of commercial three-electrode cell setup [7]. 
In-house three electrode cells utilized FTO glass and aluminum sheet rectangles for working electrodes. As can be seen in the schematic in Figure B.5, the electrodes hang in close proximity to each other. The working electrode was a $2 \times 4.5 \mathrm{~cm}$ rectangle with a $2 \times 2 \mathrm{~cm}$ square of electrode film. The reference and counter electrodes were lithium metal. The three electrodes were immersed in electrolyte, and the cell was sealed.

The cell casing was a specimen container with a double locking twist top. To seal the cell, the top is tightened then parafilm was added tightly. Upon removal from the glovebox, aluminum foil tape and hot glue were used to seal completely. The sealed cell was allowed to reach equilibrium for 4 hours before testing.

Two-electrode Swagelok-type cells are constructed similarly to the commercial three-electrode setup (Figure B.6). A base, which also acts as the contact for the working electrode holds all of the components and is connected to the cap with a threaded Teflon coupling. Inside of the cell a lithium metal disc is used as the counter electrode, with an electrolyte soaked separator placed over top. A guide is then inserted into the cell to prevent the electrode from shifting and breaking contact with the counter electrode. The working electrode (10 $\mathrm{mm} \mathrm{disc})$ is inserted into the cell with the substrate facing up, and a metal contact is placed on top to provide connection between the working electrode and the cap. The cap is screwed on tightly and the seams of the cap and coupling are sealed with petroleum jelly to prevent air from entering the cell and allowed to reach equilibrium for 4 hours before testing.

\section{B.4 Physical Characterization Methods}

\section{B.4.1 Brunauer-Emmett-Teller (BET) Surface Area}

Analyses of both powders and films were performed using a Micrometrics TriStar 3000 apparatus under nitrogen controlled atmosphere. A 55 point specific surface 


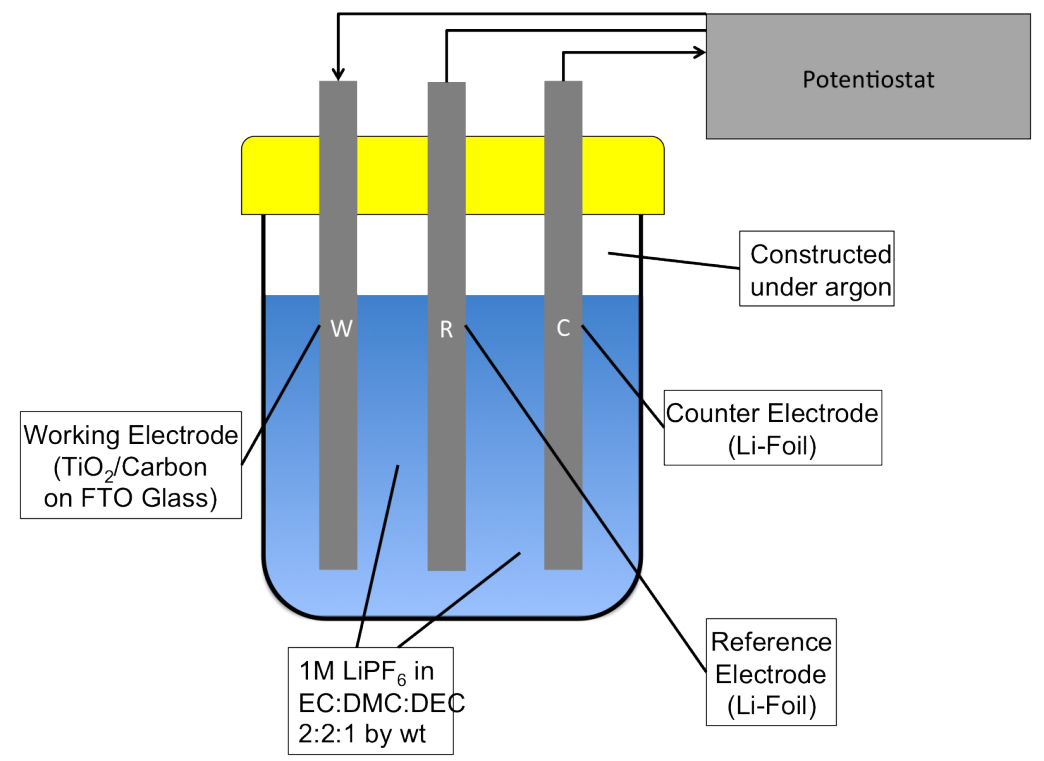

Figure B.5: Schematic of in-house three electrode cell setup.

area and pore size analysis was completed. Powder and film samples were weighed to $100 \mathrm{mg}$, and degassed under nitrogen overnight prior to nitrogen physisorption. For powders, the degassing temperature was $70^{\circ} \mathrm{C}$. Standard method films were degassed at $120^{\circ} \mathrm{C}$, while the novel method films were degassed at $300^{\circ} \mathrm{C}$. Powder samples were analyzed as prepared (dried and ground), while films were applied to untreated aluminum foil and peeled off. The pieces removed from the foil were broken down to fit into sample holder, but kept large enough to minimize the effects of sample prepartion on the results.

\section{B.4.2 X-Ray Diffraction}

X-Ray Powder Diffraction analysis was carried out on a Philips PW1710 powder diffractometer. $\mathrm{Cu}-\mathrm{K} \alpha$ radiation with a wavelength of $1.506 \AA$ was employed. The diffractometer used a voltage and current of $40 \mathrm{kV}$ and $40 \mathrm{~mA}$, respectively. Samples were scanned between $20^{\circ}$ and $90^{\circ}(2 \theta)$ with a $0.05^{\circ}$ step and a 7 sec- 


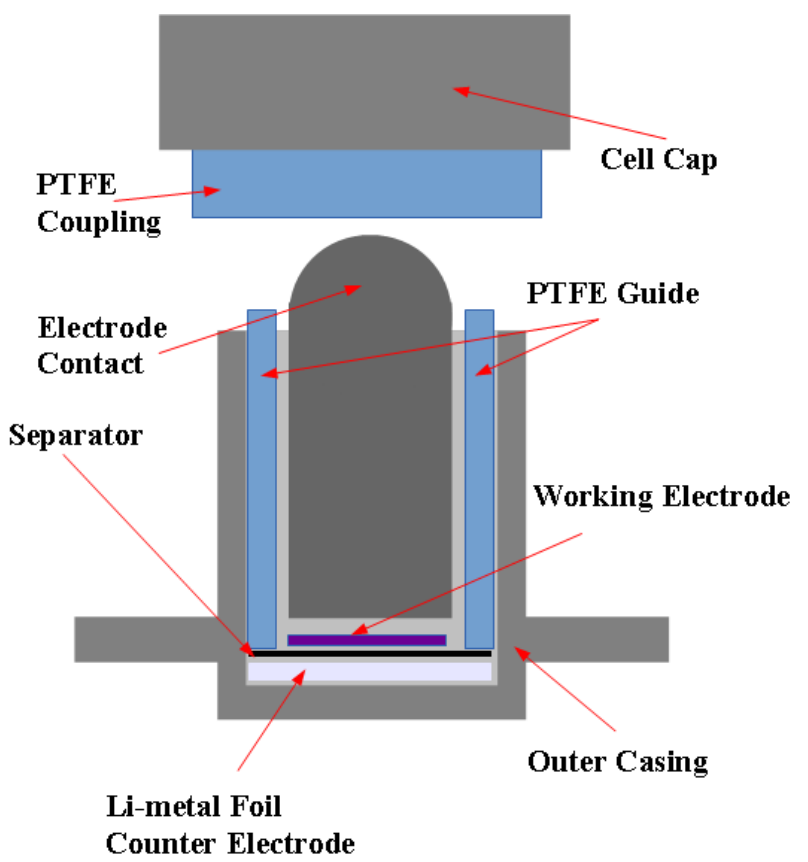

Figure B.6: Schematic of Swagelok-type cell set up.

ond acquisition time per step. For the $\mathrm{TiO}_{2}$ powders, samples were ground and packed into metal sample holders designed for powder analysis. A Bruker D8 Discover Diffractometer equipped with a copper target $\left(\lambda_{C u K \alpha}=0.15406 \mathrm{~nm}\right)$ was used to characterize aqueous synthesized anatase. $\mathrm{TiO}_{2}$ films were deposited on glass microscope slides and characterized as prepared. The patters obtained for both the powder and film samples were compared to the JCPDS reference data of anatase (\#00-021-1272), rutile (\#00-021-1276), and brookite (\#00-016-0617). Lithiated films were compared to $\mathrm{Li} 0.5 \mathrm{TiO}_{2}$. The patterns and parameters are seen in Figure B.7 and Tables A.1-A.4 in Appendix A, respectively. 

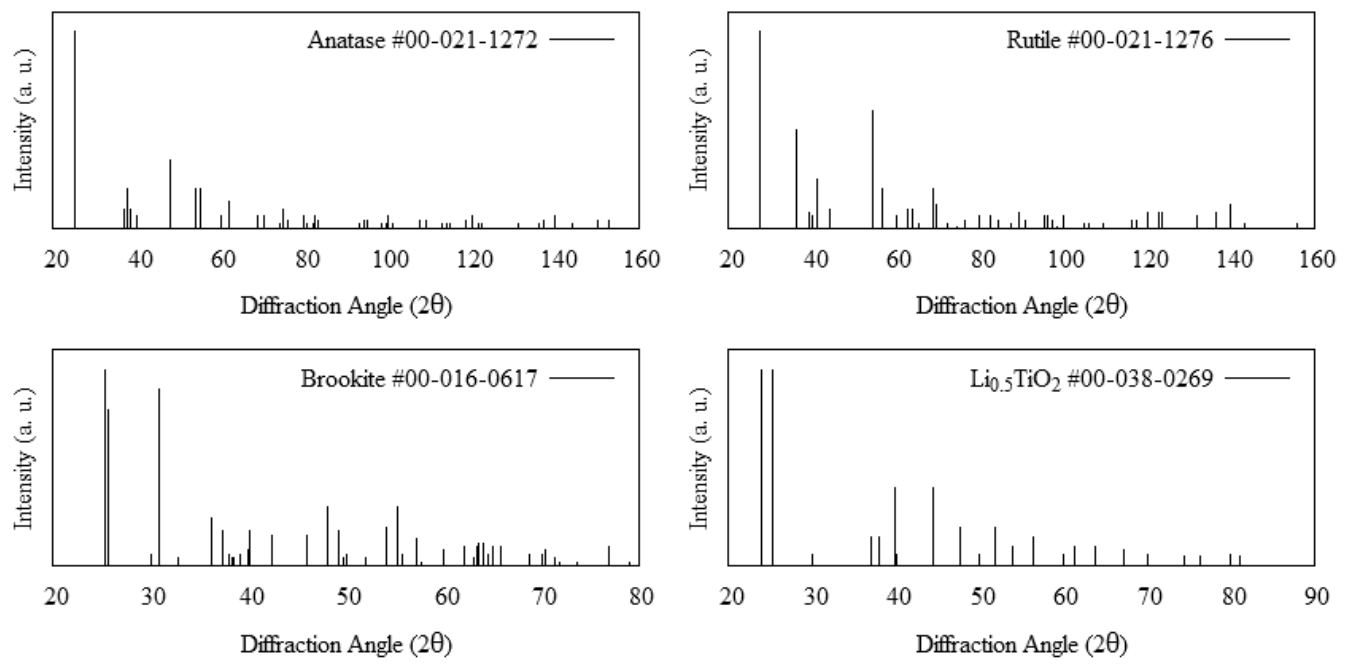

Figure B.7: JCPDS X-ray diffraction patterns of anatase (\#00-021-1272), rutile (\#00-021-1276), brookite (\#00-016-0617) and $\mathrm{Li}_{0.5} \mathrm{TiO}_{2}$ (\#00-038-0269)

\section{B.4.3 Scanning Electron Microscopy}

Physical characterization was done with a Hitachi SU-8000 cold field-emission scanning electron microscope (Hitachi, Rexdale, Canada) equipped with a Xmax silicon drift detector (SDD) as the energy dispersive spectrometer (EDS) and an electron backscatter diffraction (EBSD) Nordlys II system, both provided by Oxford Instruments (Oxford Instruments, Concord, US). The EBSD system was composed of charge-coupled device (CCD) camera of $1344 \times 1024$ pixels and a phosphorescent screen of $38 \times 27 \mathrm{~mm}^{2}$ to acquire the Kikuchi pattern. The CCD camera was used with a $4 \times 4$ binning. The diffuse background was acquired with a large number of grains and subtracted to the raw pattern by flat fielding.

Secondary electrons (SE) and scanning transmission electron microscopy (STEM) imaging were used in this work. SE imaging was carried out at an accelerating voltage of $0.5 \mathrm{kV}$ using the deceleration mode of the microscope. In this mode, a 
voltage is applied to the sample to decelerate the primary electrons before hitting the surface (landing voltage). Because the primary electron energy (before deceleration) is higher than the landing energy (after deceleration), the probe size remains small at very low landing voltages (down to $100 \mathrm{eV}$ ) and provides a high spatial resolution. The signal was then collected with two high efficiency through-the-lens detectors (upper and top detectors). STEM imaging was carried out at an accelerating voltage of $30 \mathrm{kV}$. The transmitted electrons (TE) were collected by means of an Yttrium Aluminum Garnet (YAG) type scintillator placed at the bottom of the specimen chamber, below the thin specimen. A set of apertures were used to select the collection semi-angle for bright-field (BF) imaging. In this work, a $10 \mathrm{mrad}$ semi-angle was used.

Transmission electron forwardscatter diffraction (t-EFSD) in the SEM was used to characterize the crystallography of the $\mathrm{TiO}_{2}$ blend [8--10]. In this technique, a thin specimen is placed above the commercial EBSD camera and a Kikuchi pattern is recorded. The pattern is composed of pairs of Kikuchi lines which are representative of the angular distribution of the specific Bragg diffracted transmitted electron [11]. Medians of the parallel Kikuchi lines are projections of the lattice planes on the phosphorescent screen of the camera. The phase identification is then automatically carried out by the conventional EBSD software by comparing simulated patterns of expected phases with the experimental pattern [12]. In this work, the HKL Channel 5 package was used, including the acquisition software Flamenco. A homemade specimen holder was used to perform simultaneously t-EFSD, STEM-BF imaging and $\mathrm{x}$-ray microanalysis by EDS and additional information can be found in Brodusch et al. [10].

The thin specimen used for STEM imaging and t-EFSD was prepared as follows: the coating was scraped off from the glass substrate, embedded in LR White resin (Electron Microscopy Sciences, Hatfield, US) and cured in a vacuum oven at $60^{\circ} \mathrm{C}$ for 48 hours. After trimming, the polymerized block was cut with a diamond knife with a LKB Ultrotome NOVA (LKB, Bromma, Sweden) ultramicrotome. The 
sections were retrieved on a TEM copper grid with a lacy carbon film. The sections thicknesses were between 70 and $90 \mathrm{~nm}$ and determined by the resin color under reflected light (silver to gold). To prevent carbon contamination in the specimen chamber by the organic carbon at the specimen surface, the samples were treated for 20 minutes in a Hitachi ZoneSEM ozone cleaner (Hitachi High Technologies, Rexdale, Canada) [13].

\section{B.4.4 Transmission Electron Microscopy}

A Philips CM200 Transmission Electron Microscope (TEM) was used at 200 $\mathrm{kV}$ to determine particle morphology and crystal structure. TEM specimens were prepared by depositing particles on a copper grid. A solution of sample particles in isopropanol was made. The particles in solution were deposited on the grid with an eye-dropper, and left to dry at room temperature for several minutes as the isopropanol evaporated. Selected-Area Electron Diffraction (SAED) for crystal structure analysis was conducted using a double-tilt specimen holder (2 different rotation axes with a maximum tilt angle of $\sim 20^{\circ}$ ).

\section{B.5 Electorchemical Characterization Methods}

\section{B.5.1 Cyclic Voltammetry}

Cyclic voltammetry was performed on a Biologic VSP Potentiostat/Galvanostat/EIS system using three-electrode cells constructed in an argon-filled glovebox. Titania anodes were the working electrodes, and lithium metal acted as the reference and counter electrodes. $\mathrm{LiPF}_{6}$ salt in EC:DMC:DEC was used as the electrolyte. The voltage was cycled between $3 \mathrm{~V}$ and $1 \mathrm{~V}$. The cycling rate depended on the test performed. For general lithiation and delithiation, cycling occurred at a rate of $0.1 \mathrm{mV} \mathrm{s}^{-1}$. For determination of pseudocapacitive properties, the samples were cycled at rates from 0.1 to $50 \mathrm{mV} \mathrm{s}^{-1}$. For each rate, the sample was cycled three 
times and the lithiation peak height was averaged.

\section{B.5.2 Galvanostatic Cycling}

Galvanostatic cycling was performed using both three-electrode cells and two electrode half cells (coin cells). In all cases, titania anodes were used as the working electrode and lithium metal was used as the reference and counter electrodes, and $\mathrm{LiPF}_{6}$ salt in EC:DMC:DEC was used as the electrolyte. Cycling was performed using a Biologic VSP Potentiostat/Galvanostat/EIS system.

\section{B.5.2.1 Charge/Discharge Testing}

Charge discharge testing was performed on both two- and three-electrode cells. The cutoff voltages were $3 \mathrm{~V}$ (upper cutoff) and either 1 or $1.2 \mathrm{~V}$ depending on the material and fabrication method used--P25 fabricated using the novel method, and standard methods were cutoff at $1 \mathrm{~V}$. Charge and discharge were performed at $0.16 \mathrm{~A} \mathrm{~g}^{-1}$ (equivalent of $1 \mathrm{C}$ rate for titania). For cutoff of $1.2 \mathrm{~V}$, cells were cycled 100 times, while cells cycled with $1 \mathrm{~V}$ cutoff were cycled 11 times.

Cells were allowed to reach equilibrium, then anode lithiated with a current of $-168 \mathrm{~mA} \mathrm{~g}^{-1}$. The negative current caused lithium ions to flow from the lithium source into the pristine electrode material. The cells incurred a voltage drop until they reached the cutoff voltage, and then the current was reversed to $168 \mathrm{~mA} \mathrm{~g}^{-1}$ for delithiation. The cell underwent an increase in voltage until it reached the upper cutoff voltage. This was one cycle.

\section{B.5.2.2 Rate Capability}

Rate capability was performed on two-electrode half cells. The cutoff voltages used were $3 \mathrm{~V}$ (upper) and $1 \mathrm{~V}$ (lower). In this test, the cells were cycled at various

rates. First they started with 10 cycles at $168 \mathrm{mAg}^{-1}$. Then they were cycled 5 times at each of the other rates. The rate capability testing was performed at 168 , 
$335,840,1600$ and $5050 \mathrm{~mA} \mathrm{~g}^{-1}$. This is equivalent to $1 \mathrm{C}, 2 \mathrm{C}, 5 \mathrm{C}, 10 \mathrm{C}$, and $30 \mathrm{C}$ rates.

\section{B.5.3 Electrochemical Impedance Spectroscopy}

Electrochemical Impedance Spectroscopy was performed with a three-electrode. All measurements were taken using a Biologic VSP Potentiostat/Galvanostat/EIS system. EIS was performed in the frequency range from 100,000 to $0.01 \mathrm{~Hz}$, and A.C. modulation was controlled at $5 \mathrm{mV}$. EIS was performed at various points throughout the lithiation and delithiation. Lithium ions were intercalated into the $\mathrm{TiO}_{2}$ by discharge process at a fixed current density of $0.1 \mathrm{~mA} \mathrm{~cm}^{-2}$. The cell was given various designated cutoff potentials (e.g. 2.7, 2.4, 1.7, 1.3, etc.) versus $\mathrm{Li} / \mathrm{Li}^{+}$. The cells were kept at open-circuit condition for 1 hour before performing impedance tests to ensure equilibrium within the cell.

\section{B.6 References}

[1] A. Yasin, F. Guo, G. P. Demopoulos, Materials Research Society Spring Meeting (2013).

[2] C. Charbonneau, R. Gauvin, G. P. Demopoulos, J. Cryst. Growth 312, 86 (2009).

[3] C. Charbonneau, R. Gauvin, G. P. Demopoulos, J. Electrochem. Soc., 158, H224 (2011).

[4] B. Ohanti, O. O. Prieto-Mahaney, D. Li, R. Abe, J. Photoc.Photobio., 216, 179 (2010).

[5] S. Ito, P. Chen, P. Comte, M. K. Nazeeruddin, P. Pechy, M. Grätzel, Prog. Photovoltaics, 15, 603 (2007).

[6] K.-E. Lee, C. Charbonneau, G. Shan, G. P. Demopoulos, R. Gauvin, JOM, 61, 52 (2009). 
[7] Hohsen Corporation, Schematic of 3-Electrode Cell, http://www.hohsen.co.jp/en/products/detail.php?id=3.

[8] R. R. Keller and R. H. Geiss, J. Microsc., 245, 245 (2012).

[9] P. W. Trimby, Ultramicroscopy, 120, 16 (2012).

[10] N. Brodusch, H. Demers, R. Gauvin, J. Microsc., 250, 1 (2013).

[11] S. Zaefferer, Ultramicroscopy, 107, 254 (2007).

[12] S. I. Wright, in Electron Backscatter Diffraction in Materials Science, A. J. Schwartz, M. Kumar, B. L. Adams, Editors, p 51 Plenum, New York (2000).

[13] C. Soong, P. Woo, D. Hoyle, Microsc. Today, 20(6), 44 (2012). 



\section{Appendix C}

\section{Electrochemical Testing of Novel and Standard P25 Electrodes}

This section provides extended testing on P25 electrodes to fully understand the differences in electrochemical performance between the standard and novel fabricated electrodes. In Chapter 4, P25 was applied to FTO glass and cycled 11 times (formation plus 10 cycles). There was a strong correlation between the fabrication process and the electrochemical properties, however, the data was limited, and did not look at extended cycling comparisons. This section looks at the two electrodes on aluminum foil and cycled in two electrode cells to examine the properties after 100 cycles at $1 \mathrm{C}$ rate.

\section{C.1 Experimental}

The electrodes were fabricated using $\mathrm{P} 25 \mathrm{TiO}_{2}$ (Evonik Aeroxide ${ }^{\circledR}$ ) as active material, acetylene black as conductive additive, and Polyvinylidene fluoride (PVDF; HSV 900, Arkema) in n-methyl 2-pyrrolidone (NMP; Sigma Aldrich) solvent as a binder. For the standard fabrication method, the electrodes are comprised of 80 $\mathrm{wt} \%$ active material, $10 \mathrm{wt} \%$ conductive additive, and $10 \mathrm{wt} \%$ binder. In order to maintain consistency in the conductive additive, the novel fabrication method was performed using $90 \mathrm{wt} \%$ active material and $10 \mathrm{wt} \%$ conductive additive. The two pastes were doctor bladed onto aluminum foil that was pre-treated with $1 \mathrm{M}$ $\mathrm{NaOH}$ for 10 seconds. The standard method electrode was dried for 24 hours at $120^{\circ} \mathrm{C}$ and the novel method electrode was dried at $80^{\circ} \mathrm{C}$ and then sintered with 
the profile seen in Appendix B.

Electrochemical testing was performed via a BioLogic VSP Potentiostat/Galvanostat/EIS system using Swagelok-type half cells assembled in an argon-filled glovebox. The $\mathrm{TiO}_{2}$ electrodes were the working electrodes, with lithium metal as the counter electrode. Celgard polypropylene separators were used and a solution of $1 \mathrm{M} \mathrm{LiPF}_{6}$ in EC:DMC:DEC (2:2:1 by weight) mixed solution (BASF) was used as the electrolyte. A current of $168 \mathrm{~mA} \mathrm{~g}^{-1}$ (representative of $1 \mathrm{C}$ rate) was used for both charging and discharging. Cells were first discharged to a cutoff voltage of $1 \mathrm{~V}$ to insert $\mathrm{Li}$-ions into pristine $\mathrm{TiO}_{2}$, and then charged to a cutoff voltage of $3 \mathrm{~V}$.

\section{C.2 Results}

As seen in Chapter 3, the novel fabrication method produced electrodes that had excellent cycling capacity and capacity retention compared to electrodes fabricated with the standard method. However, this testing was performed with FTO glass, in a three-electrode cell in which the electrodes were hung in the electrolyte. This subjected the electrode films to external forces, namely gravity, which meant that the electrode could not be cycled past what was performed. Presently, aluminum foil is used as the substrate for the electrode films, allowing for the cells to be used in a two-electrode setup where the working and counter electrodes are pressed against each other (with a separator between them to prevent short-circuit), removing external forces that were seen in the three-electrode cells.

Figure C.1 shows the cycling data for electrodes fabricated with the novel and standard fabrication methods. As seen previously, the novel method electrode initially has a reversible capacity of approximately $185 \mathrm{~mA} \mathrm{~h} \mathrm{~g}^{-1}$, and over the next ten cycles endures a slight drop in capacity to $160 \mathrm{~mA} \mathrm{~h}^{-1}$ but an increase in efficiency to almost $100 \%$. In contrast, the standard electrode has an initial reversible capacity of $\sim 150 \mathrm{~mA} \mathrm{~h} \mathrm{~g}^{-1}$ and a much larger capacity drop to $60 \mathrm{~mA} \mathrm{~h} \mathrm{~g}^{-1}$ after 10 cycles. With the aluminum foil substrate, the standard method electrode has a much 
higher coulombic efficiency, which reaches almost 100\% (like the novel method electrode), but it requires more cycles to reach that equilibrium. After the initial capacity drop, the standard method electrode stabilizes and cycles continuously with a reversible capacity of $60 \mathrm{~mA} \mathrm{~h}^{-1}$.

The novel method electrode, however, shows a constant decrease in capacity. While that capacity remains much higher than the standard method $\left(135 \mathrm{~mA} \mathrm{~h} \mathrm{~g}^{-1}\right.$ after 100 cycles) an maintains its excellent coulombic efficiency, there is a visibly trend of decreasing capacity. This may have to do with particle coarsening during the sintering step. Because the particles start out at $\sim 21 \mathrm{~nm}$ in diameter, they will not grow significantly, however, by connecting them, there is some loss in surface area. This, couple with a small amount of irreversible capacity could lead to the gradual decrease in capacity seen over 100 cycles. However, after approximately 80 cycles, the capacity seems to plateau. This may mean that the novel method electrode with P25 as its active material requires more cycles before it reaches a stable capacity. It is unknown at this time, why this may occur, but it is likely that the expansion and contraction of the active material (though only $4 \%$ overall) may effect the capacity slightly causing the drop seen over these cycles.

Nonetheless, the significant capacity difference between the standard and novel methods show that the removal of binder provides an excellent platform for higher capacity electrodes. While this data is preliminary, as P25 would not be used as an active material due to the large size of the particles, it provides excellent insight into the effects of sintering and material dispersion on the performance of binder-free electrodes. 


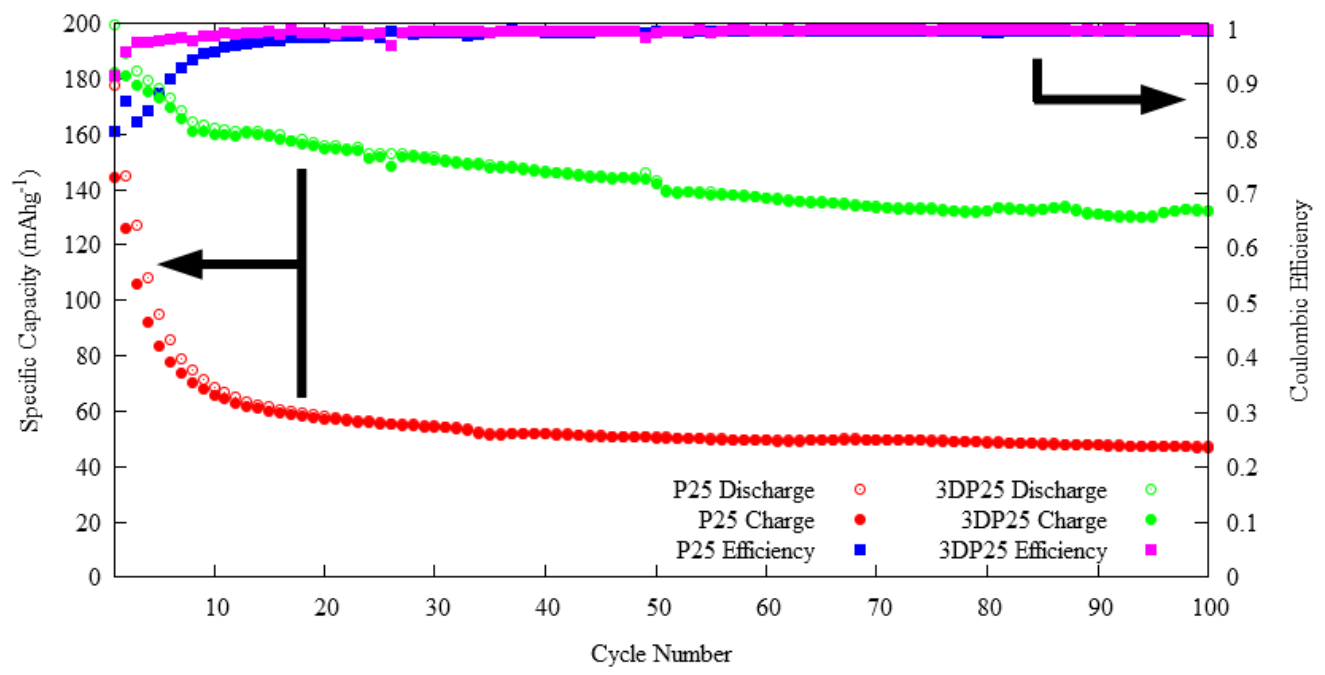

Figure C.1: Galvanostatic charge/discharge over 100 cycles between cutoff voltages of $1 \mathrm{~V}$ (discharge) and $3 \mathrm{~V}$ (charge) at $1 \mathrm{C}$ rate $\left(168 \mathrm{~mA} \mathrm{~g}^{-1}\right)$ 


\section{Appendix D}

\section{Supplementary Information for Mesoporous Brookite Nanoplatelets with Superior Lithium Intercalation Stability}

This section provides supporting data for the paper whose title is presented above. Figure D.1 shows the Reitveld refinement model for the synthesized brookite material, as well as the deconvolution of the two main peaks corresponding to the (120) and (111) planes respectively. Figure D.2 shows the N2 adsorption curve for the brookite nanoplatelet material. It depicts a type II isotherm indicative of a porous nanosolid [1]. Figure D.3 is the voltage profile for the fisrt lithiation of the mixed-phase brookite ( $73 \%$ brooktie and $27 \%$ rutile). The profile shows that there is no plateau at 1.4 volts that is a characteristic feature of rutile [2]. Figure D.4 shows the Nyquist plots of the synthesized brookite at various levels of lithiation during the formation cycle. In each plot there is a single semicircle whose first intercept is associated with the electrolyte resistance $\left(R_{e}\right)$; height is associated with the double layer capacitance $\left(C_{d l}\right)$; and diameter is associated with the charge-transfer resistance $\left(R_{c t}\right)$. Each of the plots also has a linear tail associated with the Warburg impedance, which describes diffusion related resistances. Figure D.5 shows the trend in phase angle of the Warburg element over the voltage profile. Table D.1 gives the important data associated with the EIS spectra shown in Figure D.4. Figure D.6 shows the equivalent circuit diagrams used with ZView software to fit the electrochemical impedence spectra. Figure D. 6 depicts the equivalent circuit used 
to fit the EIS data for both the pristine and delithiated brookite, while Figure D.6 is the equivalent circuit used to fit the EIS data for brookite during lithiation. Figure D.7 shows the Nyquist plots for pristine brookite and brookite that has been cycled 10 times. Each of these has 2 semicircles. The first, as with the plots in Figure D.4, has a first intercept associated with the electrolyte resistance, but the height and diameter are associated with the charge-separation capacitance and resistance that occurs with the lithium gradient in the system when the $\mathrm{TiO}_{2}$ is not lithiated. Table D. 2 gives the charge and discharge capacities and the coulombic efficiencies associated with the brookite nanoplatelets at $\mathrm{C} / 10,1 \mathrm{C}$, and $10 \mathrm{C}$ rates (corresponding to Figure $4.6 \mathrm{~b}$ of the main paper). Figure D. 8 is a zoomed-in view of the X-ray diffraction patterns for the pristine, lithiated, and delithiated brookite showing peak shifts (marked by dotted and solid lines through the apexes of the peaks) from $2 \theta=25.69^{\circ}$ to $2 \theta=25.25^{\circ}$, and the loss of the peak associated with the (121) plane of brookite, as the active material lithiates.

\section{D.1 References}

[1] J. B. Condon, Surface Area and Porosity Determinations by Physisorption: Measurement and Theory, Elsevier Science (2006).

[2] P. Kubiak, M. Pfanzelt, J. Geserick, U. Hormann, N. Husing, U. Kaiser, M. Wohlfahrt-Mehrens, J. Power Sources, 194, 1099 (2009). 

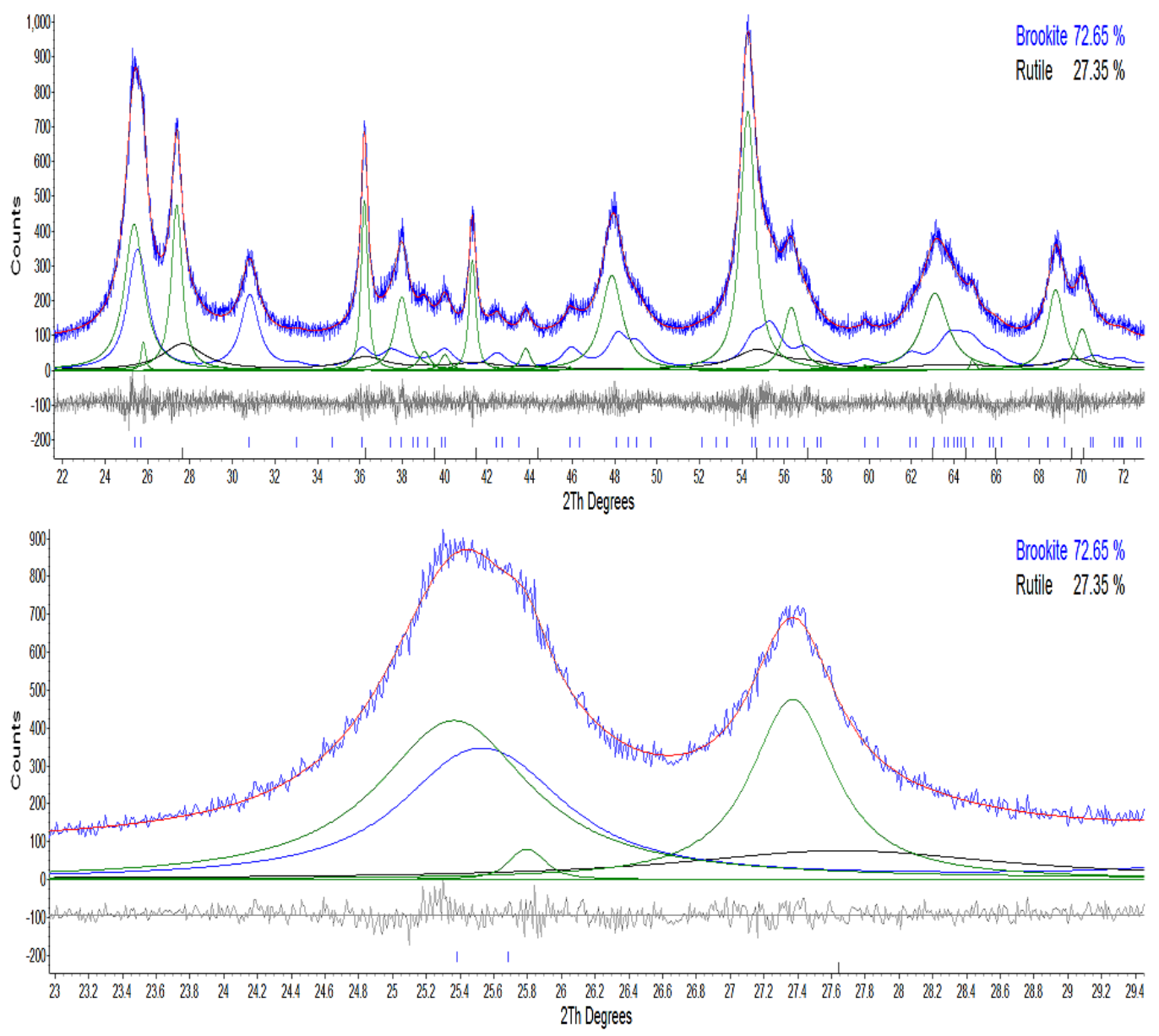

Figure D.1: Reitveld refinement of synthesized brookite (a) and the deconvolution of the two main brookite peaks (b). The individual peak convolutions are below the observed and refined patterns, and the gray pattern below the $\mathrm{x}$-axis is the refinement error. 


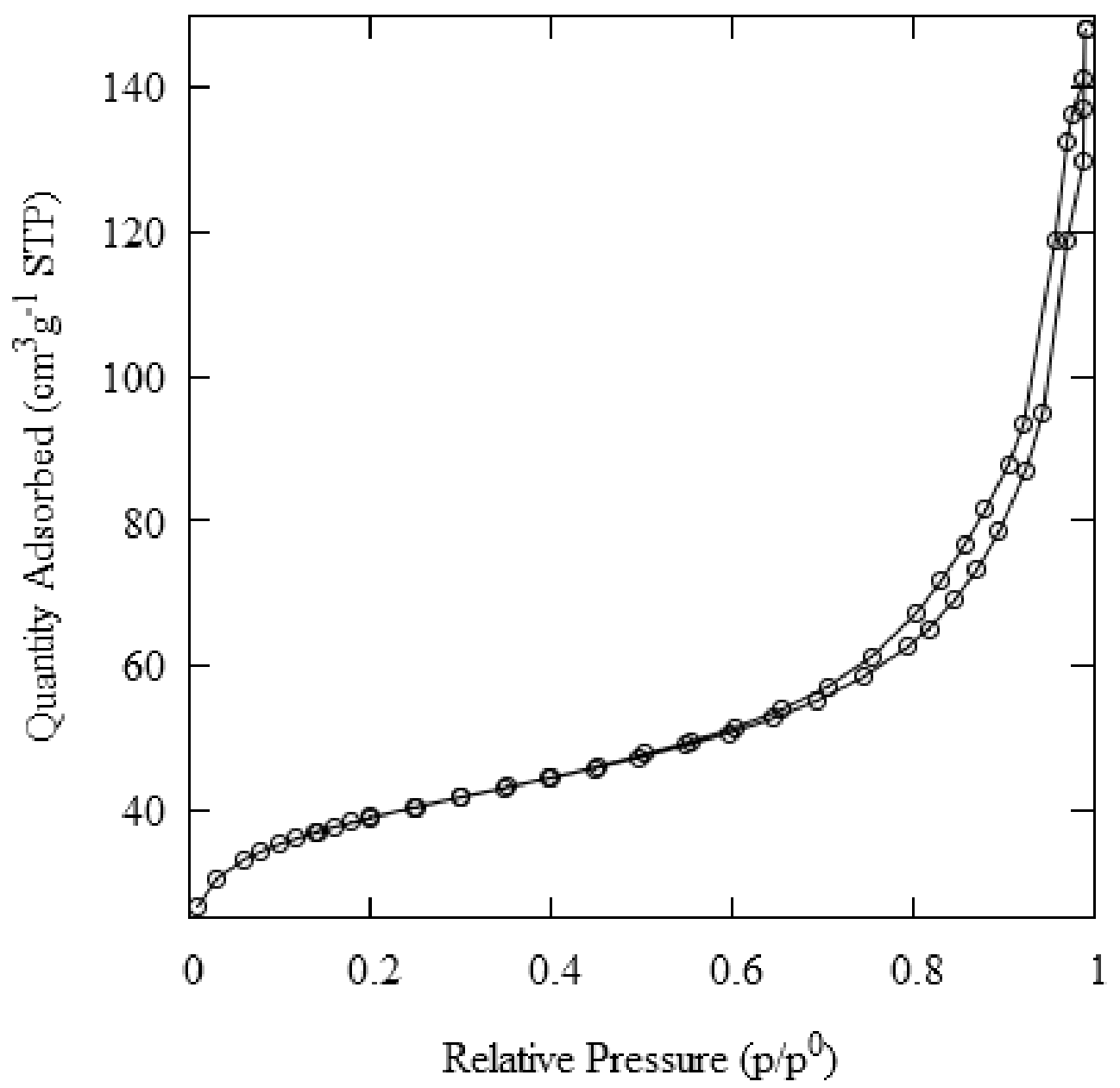

Figure D.2: The $\mathrm{N}_{2}$ isotherm for aqueous synthesized brookite 


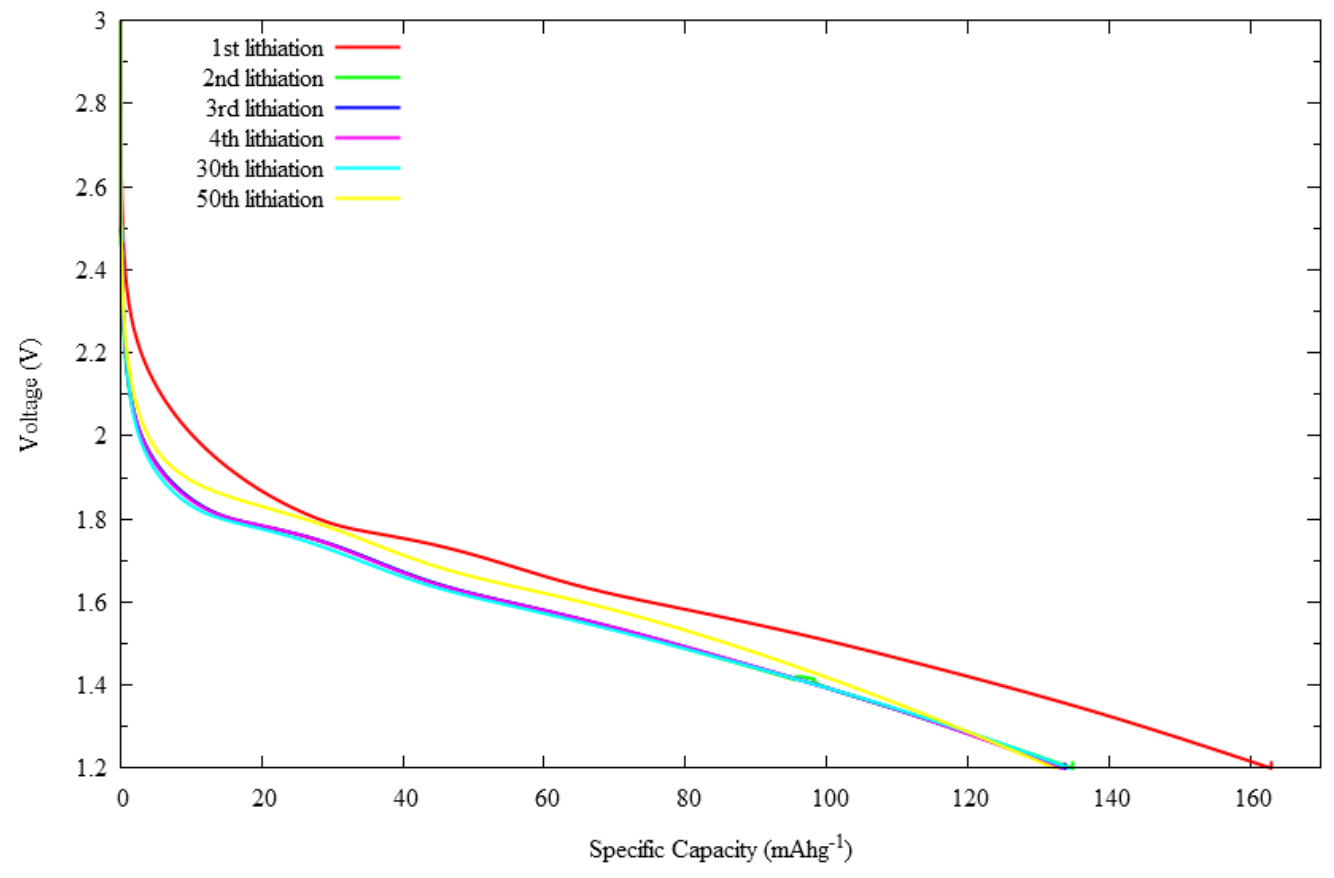

Figure D.3: Voltage profiles for the lithiation at various cycles (1-4, 30 and 50) at $\mathrm{C} / 10$ rate of the as synthesized brookite. The capacity is calculated based on the total mass of $\mathrm{TiO}_{2}$. 


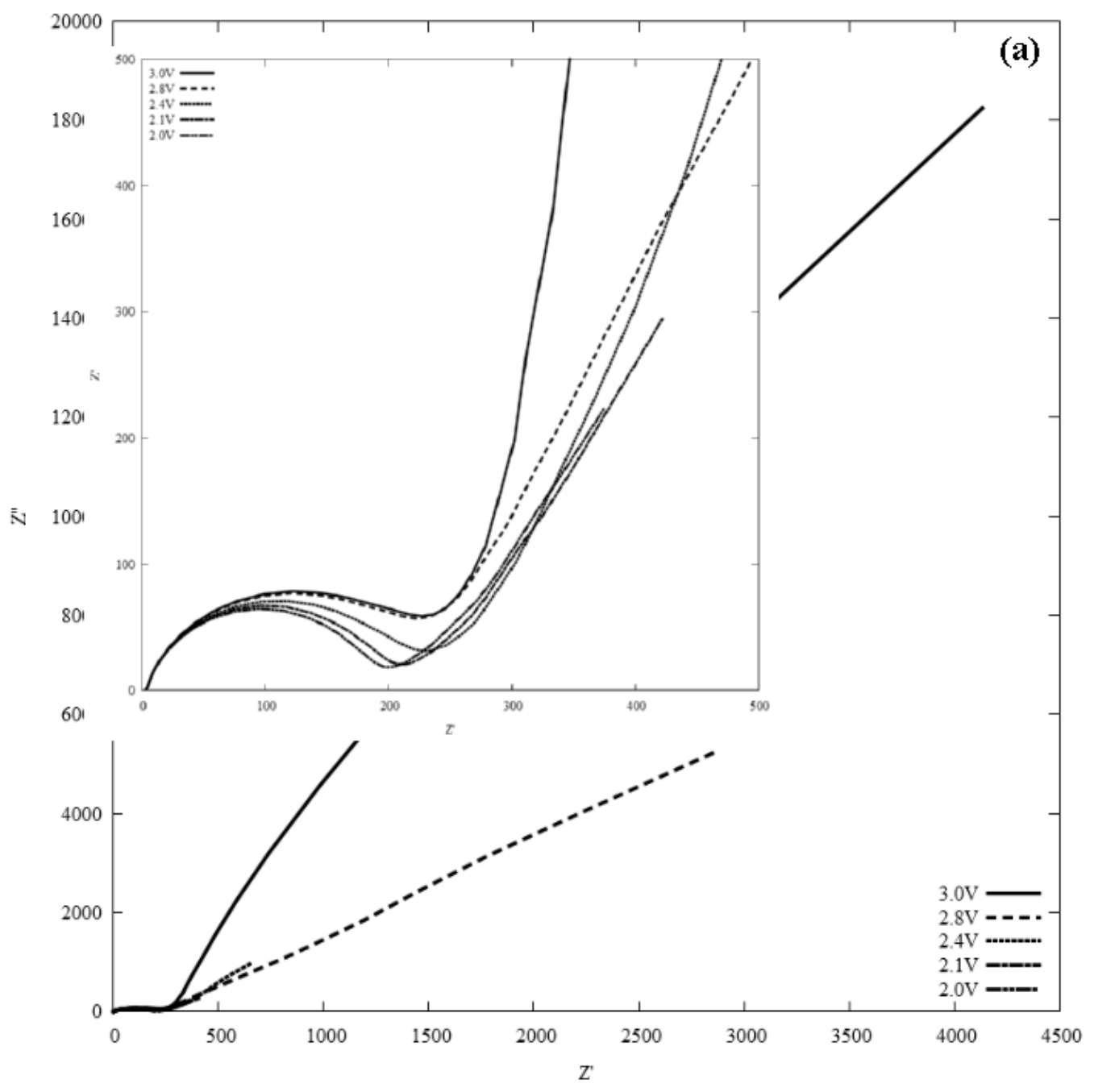

186 


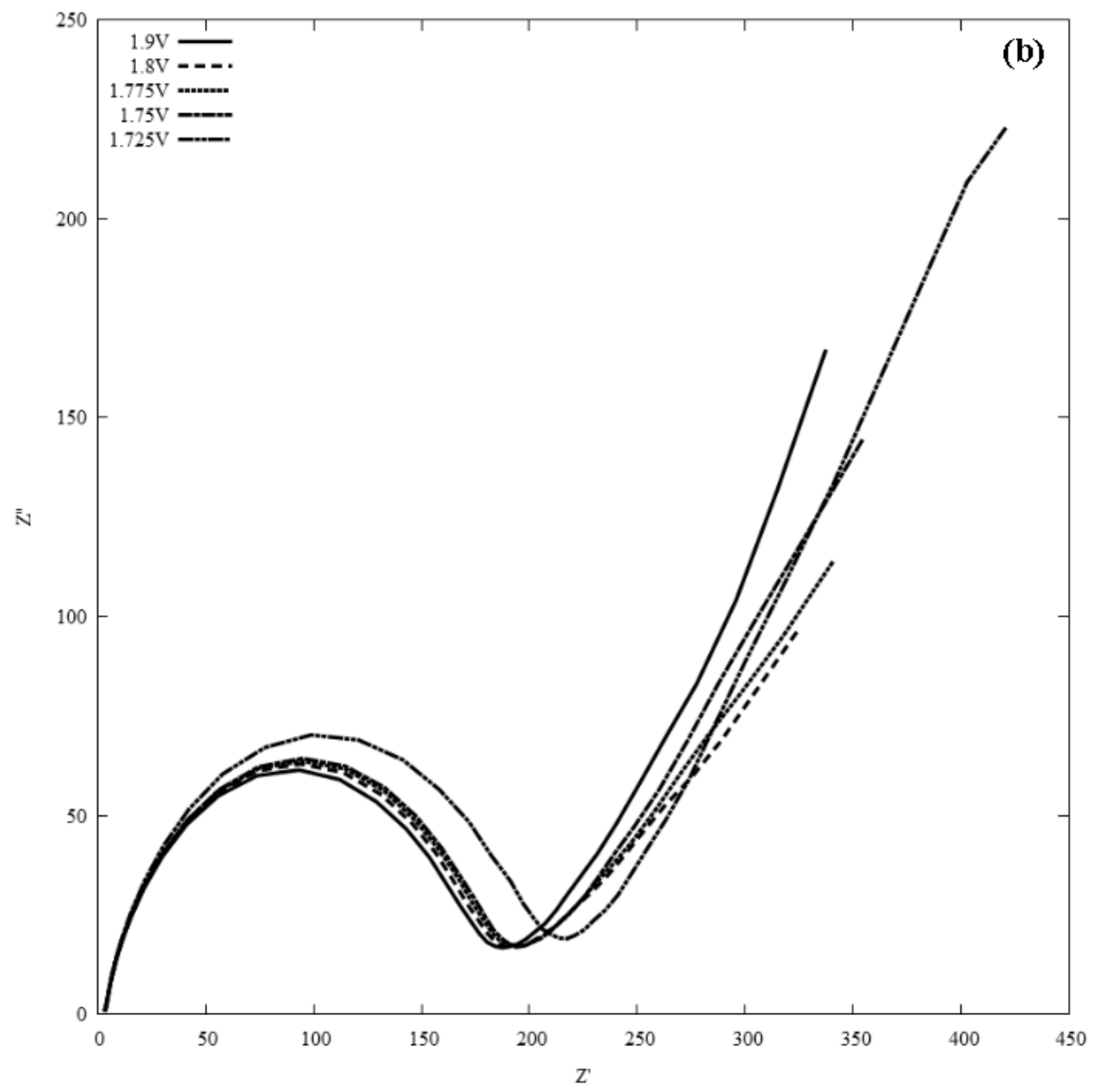




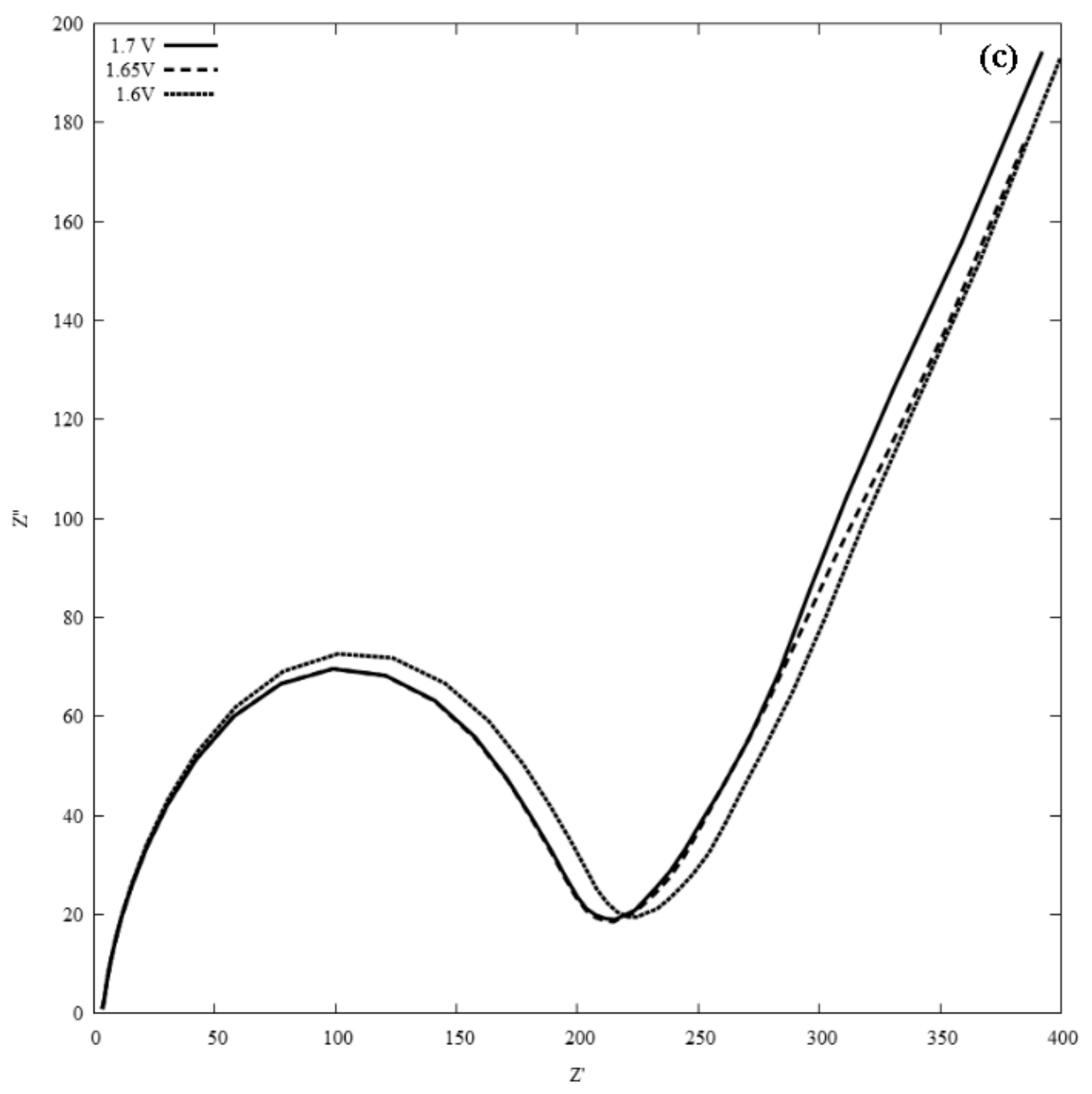




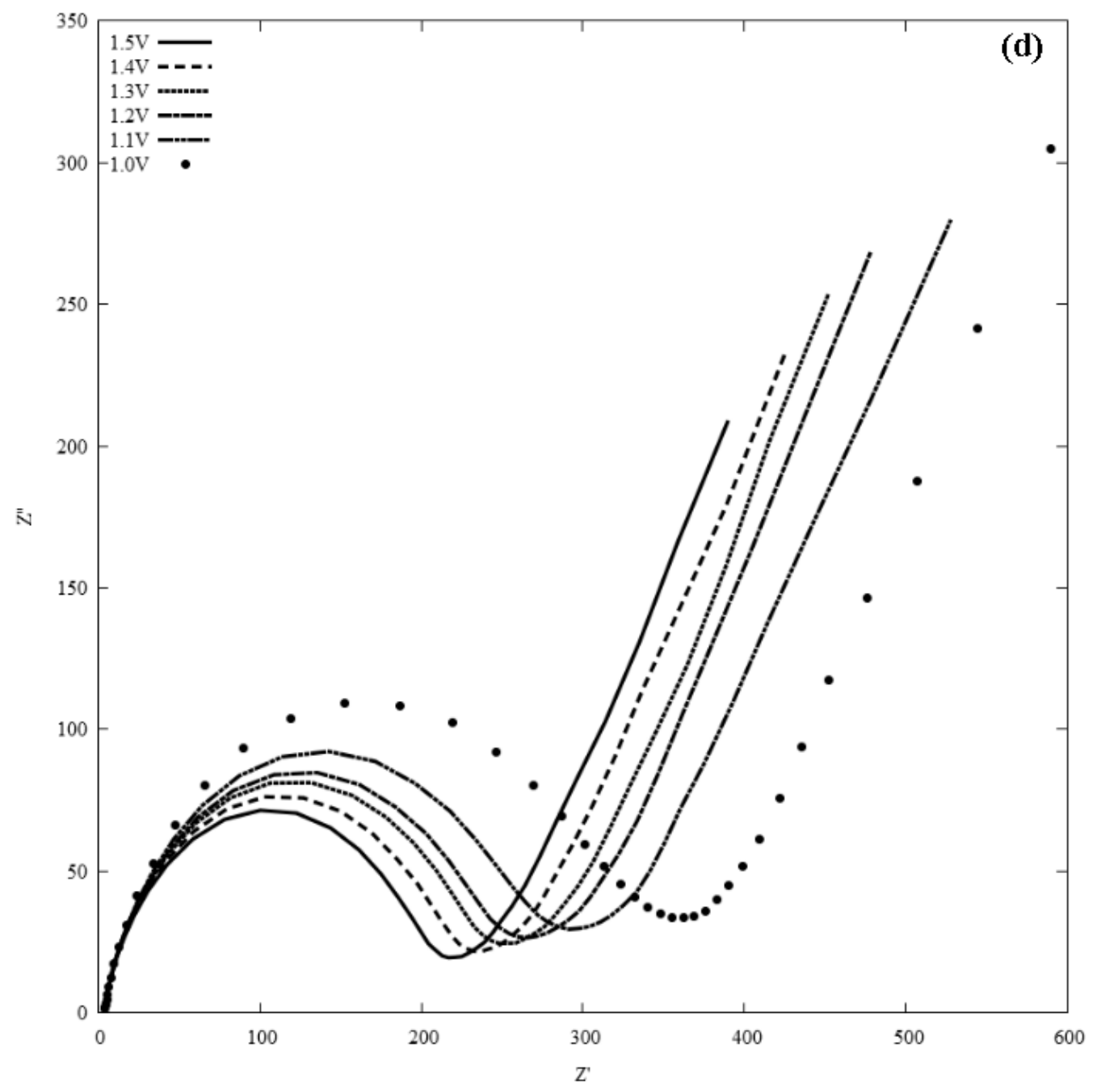

Figure D.4: Nyquist plots of brookite at various levels of lithiation during the formation step---refer to Figure 4.4 in the published paper. During the initial voltage drop from $3.0 \mathrm{~V}$ to $2.0 \mathrm{~V}$ (a) the diffusion tail length decreases. From $1.9 \mathrm{~V}$ to $1.725 \mathrm{~V}$ (b) the diffusion tail length remains constant. As the voltage $1.7 \mathrm{~V}$ to $1.6 \mathrm{~V}$ (c) the charge-transfer resistance increases while everything else remains constant. Finally as the voltage drops from $1.5 \mathrm{~V}$ to the cutoff voltage at $1 \mathrm{~V}(\mathrm{~d})$, the diffusion tail and charge-transfer resistance increase. 


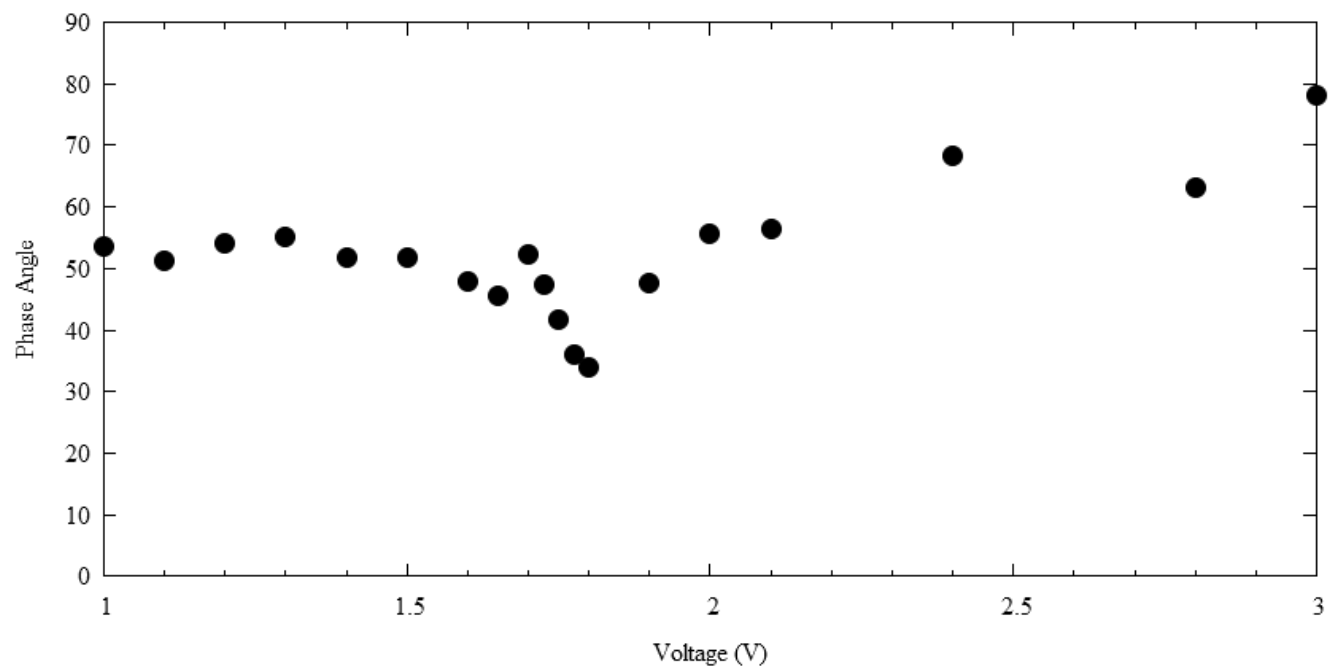

Figure D.5: Plot of $Z_{W}$ phase angle versus voltage. 
Table D.1: Important parameters associated with EIS over the formation cycle of brookite nanoplatelets.

\begin{tabular}{cccccc}
\hline Voltage $(\mathrm{V})$ & $R_{e}(\Omega)$ & $R_{c t}(\Omega)$ & $C_{d l}(F)$ & Phase Angle & $Z_{w}$ \\
\hline 3 & 3.8 & 160.9 & $1.41 \mathrm{E}-005$ & 78.02 & 18652.24 \\
2.8 & 3.394 & 186.2 & $1.15 \mathrm{E}-004$ & 63.24 & 5925.2 \\
2.4 & 3.301 & 196.5 & $1.38 \mathrm{E}-004$ & 68.22 & 1072.72 \\
2.1 & 3.265 & 180.5 & $1.07 \mathrm{E}-004$ & 56.33 & 354.65 \\
2 & 3.237 & 171.5 & $9.76 \mathrm{E}-005$ & 55.62 & 272.35 \\
1.9 & 3.133 & 158.9 & $9.31 \mathrm{E}-005$ & 47.63 & 225.52 \\
1.8 & 3.182 & 152.4 & $8.18 \mathrm{E}-005$ & 33.9 & 172.11 \\
1.775 & 3.188 & 157.2 & $8.27 \mathrm{E}-005$ & 36.11 & 193.32 \\
1.75 & 3.182 & 162.9 & $8.58 \mathrm{E}-005$ & 41.71 & 216.5 \\
1.725 & 3.22 & 182 & $8.41 \mathrm{E}-005$ & 47.51 & 301.74 \\
1.7 & 3.208 & 181 & $8.51 \mathrm{E}-005$ & 52.26 & 245.29 \\
1.65 & 3.201 & 179.9 & $8.31 \mathrm{E}-005$ & 45.61 & 246.53 \\
1.6 & 3.231 & 189.9 & $8.23 \mathrm{E}-005$ & 47.8 & 260.4 \\
1.5 & 3.215 & 189.1 & $8.68 \mathrm{E}-005$ & 51.71 & 265.52 \\
1.4 & 3.248 & 202.3 & $8.54 \mathrm{E}-005$ & 51.72 & 295.27 \\
1.3 & 3.3 & 216.5 & $8.49 \mathrm{E}-005$ & 55.2 & 306.05 \\
1.2 & 3.34 & 226.1 & $8.60 \mathrm{E}-005$ & 54.14 & 330.84 \\
1.1 & 3.535 & 246.7 & $9.15 \mathrm{E}-005$ & 51.24 & 357.96 \\
1 & 3.628 & 300 & $9.58 \mathrm{E}-005$ & 53.7 & 378.32 \\
\hline
\end{tabular}



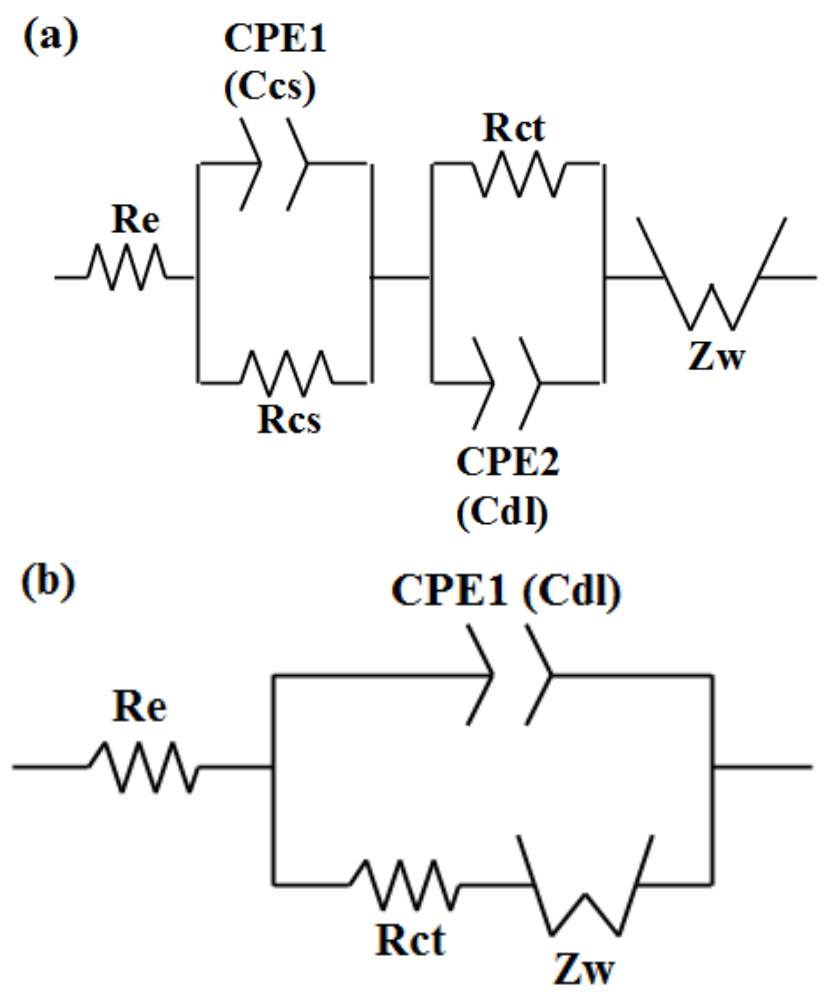

Figure D.6: Equivalent circuit for both pristine and brookite cycled 10 times (a) and equivalent circuit for brookite during lithiation (b). $R_{e}$ is the electrolyte resistance, $R_{c} s$ is the charge separation resistance, $C_{c} s$ is the charge separation capacitance (the combination of which gives the charge separation overpotential), $R_{c t}$ is the chrage transfer resistance, $C_{d l}$ is the double layer capacitance and $Z_{W}$ is the Warburg impedance. 


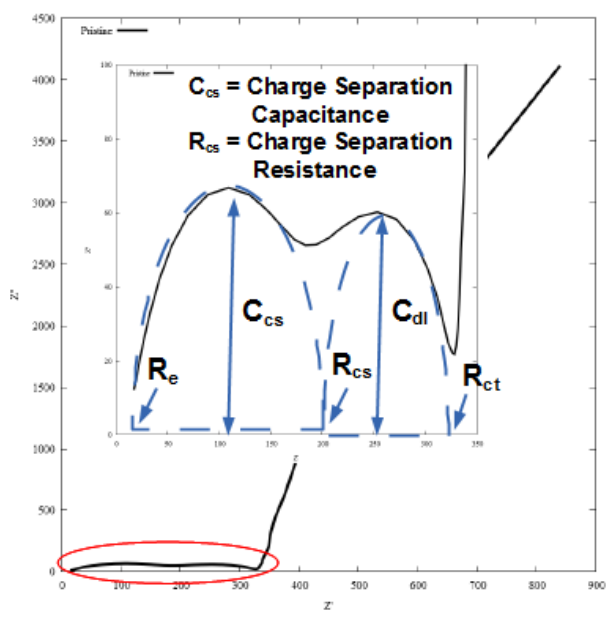

(a)

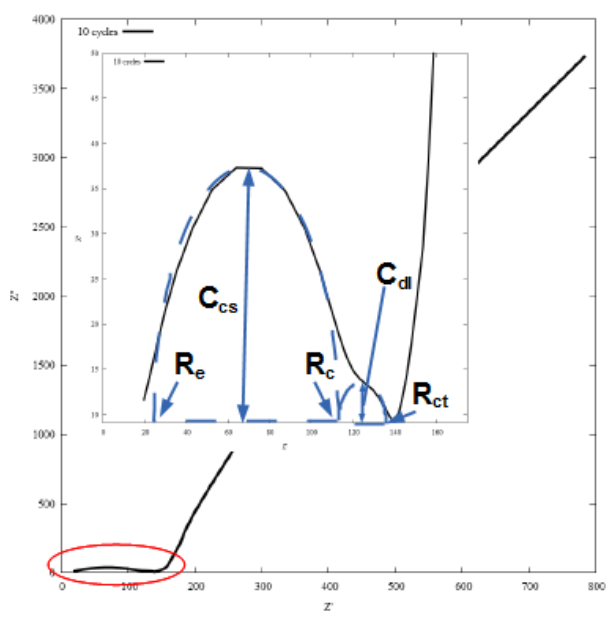

(b)

Figure D.7: Annotated Nyquist plots for pristine brookite (a) and delithiated brookite after 10 cycles (b). Re is the electrolyte resistance, $C_{c s}$ is the charge-separation capacitance, $R_{c s}$ is the charge-separation resistance, $C_{d l}$ is the double layer capacitance, and $R_{c t}$ is the charge-transfer resistance. 
Table D.2: Capacities (based on the mNP brookite fraction only) associated with the charge and discharge as well as the coulombic efficiency for brookite at $\mathrm{C} / 10,1 \mathrm{C}$, and $10 \mathrm{C}$ rate

\begin{tabular}{|c|c|c|c|}
\hline $\begin{array}{c}\text { Rate } \\
(\mathrm{C}=168 \mathrm{~mA} / \mathrm{g})\end{array}$ & $\begin{array}{c}\text { Lithiation } \\
\text { Capacity }\left(\mathrm{mA} \mathrm{h}^{-1}\right)\end{array}$ & $\begin{array}{c}\text { Delithiation } \\
\text { Capacity }\left(\mathrm{mA} \mathrm{h}^{-1}\right)\end{array}$ & $\begin{array}{l}\text { Coulombic } \\
\text { Efficiency }\end{array}$ \\
\hline $\mathrm{C} / 10$ & 223.287 & 172.836 & 0.7720377809 \\
\hline $\mathrm{C} / 10$ & 184.889 & 179.66 & 0.9717155993 \\
\hline $\mathrm{C} / 10$ & 184.592 & 183.413 & 0.9936129409 \\
\hline $\mathrm{C} / 10$ & 184.357 & 183.967 & 0.9978845392 \\
\hline $\mathrm{C} / 10$ & 181.976 & 178.975 & 0.9835127508 \\
\hline $\mathrm{C} / 10$ & 178.091 & 174.85 & 0.9818000063 \\
\hline $\mathrm{C} / 10$ & 178.451 & 175.404 & 0.9829256898 \\
\hline $\mathrm{C} / 10$ & 175.284 & 172.913 & 0.986473702 \\
\hline $\mathrm{C} / 10$ & 174.542 & 172.237 & 0.986795223 \\
\hline $\mathrm{C} / 10$ & 177.257 & 176.078 & 0.9933490209 \\
\hline $\mathrm{C} / 10$ & 178.78 & 177.501 & 0.9928473226 \\
\hline $\mathrm{C} / 10$ & 177.955 & 176.744 & 0.993193094 \\
\hline $\mathrm{C} / 10$ & 179.848 & 178.24 & 0.9910617671 \\
\hline $\mathrm{C} / 10$ & 179.592 & 178.368 & 0.993183189 \\
\hline $\mathrm{C} / 10$ & 180.406 & 178.966 & 0.9920211633 \\
\hline $\mathrm{C} / 10$ & 180.354 & 178.974 & 0.9923457628 \\
\hline $\mathrm{C} / 10$ & 180.445 & 179.096 & 0.9925204973 \\
\hline $\mathrm{C} / 10$ & 181.262 & 179.954 & 0.9927829996 \\
\hline $\mathrm{C} / 10$ & 181.126 & 179.879 & 0.9931182246 \\
\hline $\mathrm{C} / 10$ & 180.983 & 179.834 & 0.9936498641 \\
\hline $\mathrm{C} / 10$ & 182.177 & 181.024 & 0.9936678997 \\
\hline $\mathrm{C} / 10$ & 182.129 & 181.084 & 0.9942637759 \\
\hline $\mathrm{C} / 10$ & 182.749 & 181.776 & 0.9946789344 \\
\hline $\mathrm{C} / 10$ & 183.895 & 182.924 & 0.9947189885 \\
\hline $\mathrm{C} / 10$ & 182.808 & 181.905 & 0.9950608693 \\
\hline $\mathrm{C} / 10$ & 182.223 & 181.375 & 0.9953465947 \\
\hline $\mathrm{C} / 10$ & 182.625 & 181.7 & 0.9949310702 \\
\hline $\mathrm{C} / 10$ & 182.993 & 182.204 & 0.9956882304 \\
\hline
\end{tabular}




\begin{tabular}{|c|c|c|c|}
\hline $\begin{array}{c}\text { Rate } \\
(\mathrm{C}=168 \mathrm{~mA} / \mathrm{g})\end{array}$ & $\begin{array}{c}\text { Lithiation } \\
\text { Capacity }\left(\mathrm{mA} \mathrm{h}^{-1}\right)\end{array}$ & $\begin{array}{c}\text { Delithiation } \\
\text { Capacity }\left(\mathrm{mA} \mathrm{h}^{-1}\right)\end{array}$ & $\begin{array}{l}\text { Coulombic } \\
\text { Efficiency }\end{array}$ \\
\hline $\mathrm{C} / 10$ & 183.536 & 182.837 & 0.9961937527 \\
\hline $\mathrm{C} / 10$ & 183.516 & 182.701 & 0.995562925 \\
\hline $\mathrm{C} / 10$ & 183.408 & 182.691 & 0.9960892413 \\
\hline $\mathrm{C} / 10$ & 183.85 & 183.18 & 0.9963562805 \\
\hline $\mathrm{C} / 10$ & 183.411 & 182.794 & 0.9966363189 \\
\hline $\mathrm{C} / 10$ & 183.278 & 182.66 & 0.9966255706 \\
\hline $\mathrm{C} / 10$ & 183.846 & 183.256 & 0.9967940156 \\
\hline $\mathrm{C} / 10$ & 183.771 & 183.167 & 0.9967131643 \\
\hline $\mathrm{C} / 10$ & 183.561 & 183.062 & 0.9972849789 \\
\hline $\mathrm{C} / 10$ & 184.443 & 183.918 & 0.9971495058 \\
\hline $\mathrm{C} / 10$ & 183.609 & 183.157 & 0.9975400343 \\
\hline $\mathrm{C} / 10$ & 183.637 & 183.119 & 0.9971814024 \\
\hline $\mathrm{C} / 10$ & 184.117 & 183.719 & 0.9978344179 \\
\hline $\mathrm{C} / 10$ & 184.43 & 184.115 & 0.9982945568 \\
\hline $\mathrm{C} / 10$ & 184.296 & 183.978 & 0.9982697218 \\
\hline $\mathrm{C} / 10$ & 184.486 & 183.935 & 0.9970091667 \\
\hline $\mathrm{C} / 10$ & 183.339 & 182.994 & 0.9981180402 \\
\hline $\mathrm{C} / 10$ & 183.519 & 183.171 & 0.9981010032 \\
\hline $\mathrm{C} / 10$ & 184.118 & 183.593 & 0.9971462414 \\
\hline $\mathrm{C} / 10$ & 183.925 & 183.811 & 0.9993781173 \\
\hline $\mathrm{C} / 10$ & 184.504 & 184.227 & 0.9984985456 \\
\hline $\mathrm{C} / 10$ & 183.338 & 182.968 & 0.997980387 \\
\hline $1 \mathrm{C}$ & 132.143 & 131.983 & 0.9987948996 \\
\hline $1 \mathrm{C}$ & 132.691 & 132.445 & 0.9981431506 \\
\hline $1 \mathrm{C}$ & 133.452 & 133.157 & 0.9977919666 \\
\hline $1 \mathrm{C}$ & 132.896 & 132.783 & 0.999148213 \\
\hline $1 \mathrm{C}$ & 132.554 & 132.071 & 0.9963568715 \\
\hline $10 \mathrm{C}$ & 64.46 & 64.366 & 0.9985462831 \\
\hline $10 \mathrm{C}$ & 64.247 & 64.118 & 0.997996387 \\
\hline $10 \mathrm{C}$ & 63.402 & 63.319 & 0.998690997 \\
\hline $10 \mathrm{C}$ & 63.189 & 63.066 & 0.9980506264 \\
\hline $10 \mathrm{C}$ & 63.083 & 62.978 & 0.9983384925 \\
\hline
\end{tabular}




\begin{tabular}{cccc}
\hline $\begin{array}{c}\text { Rate } \\
(\mathrm{C}=168 \mathrm{~mA} / \mathrm{g})\end{array}$ & $\begin{array}{c}\text { Lithiation } \\
\text { Capacity }\left(\mathrm{mA} \mathrm{h}^{-1}\right)\end{array}$ & $\begin{array}{c}\text { Delithiation } \\
\text { Capacity }\left(\mathrm{mA} \mathrm{h}^{-1}\right)\end{array}$ & $\begin{array}{c}\text { Coulombic } \\
\text { Efficiency }\end{array}$ \\
\hline $10 \mathrm{C}$ & 62.539 & 62.403 & 0.9978259433 \\
$10 \mathrm{C}$ & 62.668 & 62.476 & 0.9969400471 \\
$10 \mathrm{C}$ & 62.43 & 62.307 & 0.9980341203 \\
$10 \mathrm{C}$ & 62.198 & 62.047 & 0.9975669123 \\
$10 \mathrm{C}$ & 62.096 & 61.945 & 0.9975769641 \\
$10 \mathrm{C}$ & 62.122 & 62.033 & 0.9985601608 \\
\hline
\end{tabular}




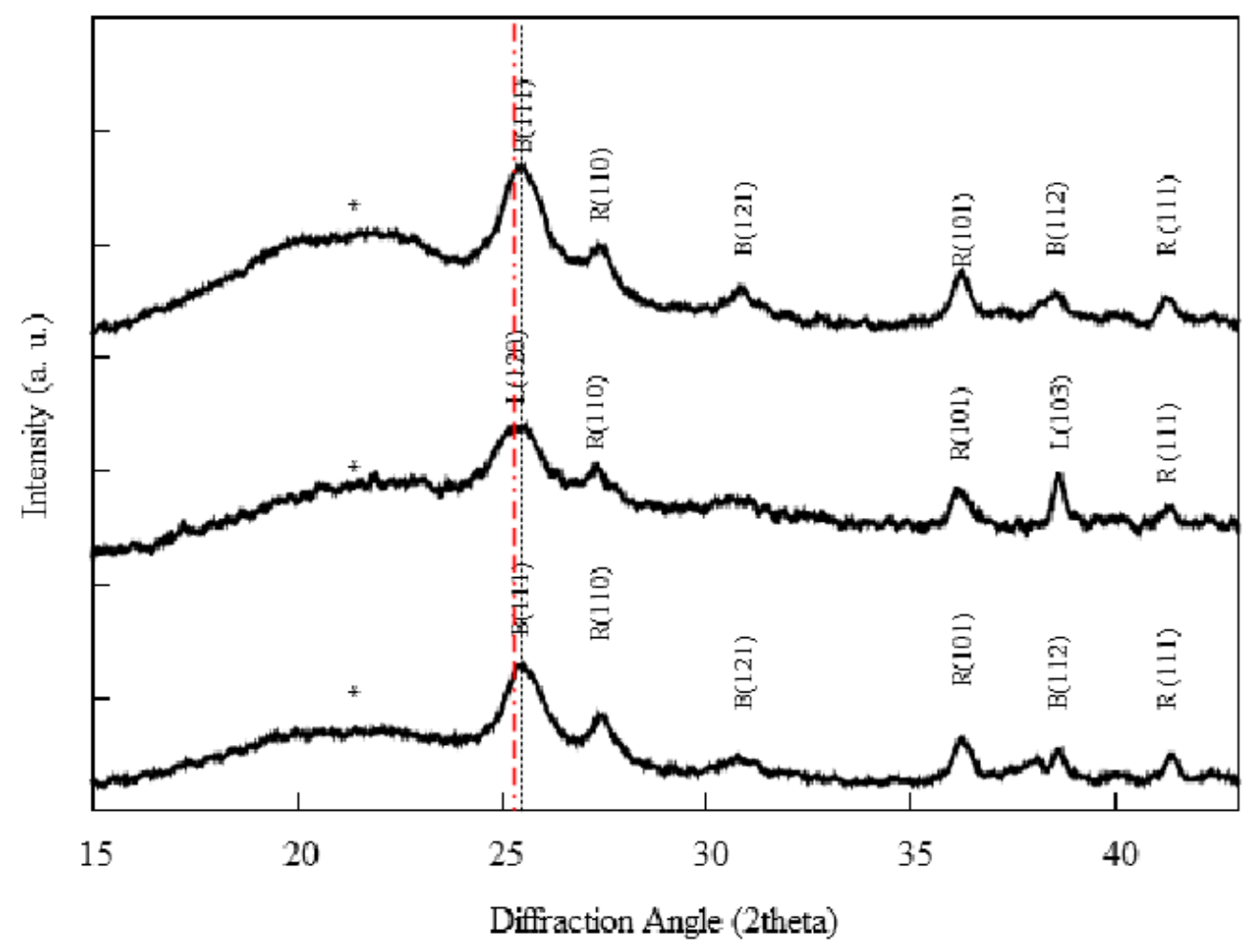

Figure D.8: Zoomed in XRD patterns of pristine (I), lithiated (II) and delithiated(III) electrodes showing peak shift and loss of 121 peak of brookite upon lithiation. The amorphous hump (indicated by "*") is caused by mounting the samples to glass slides. 


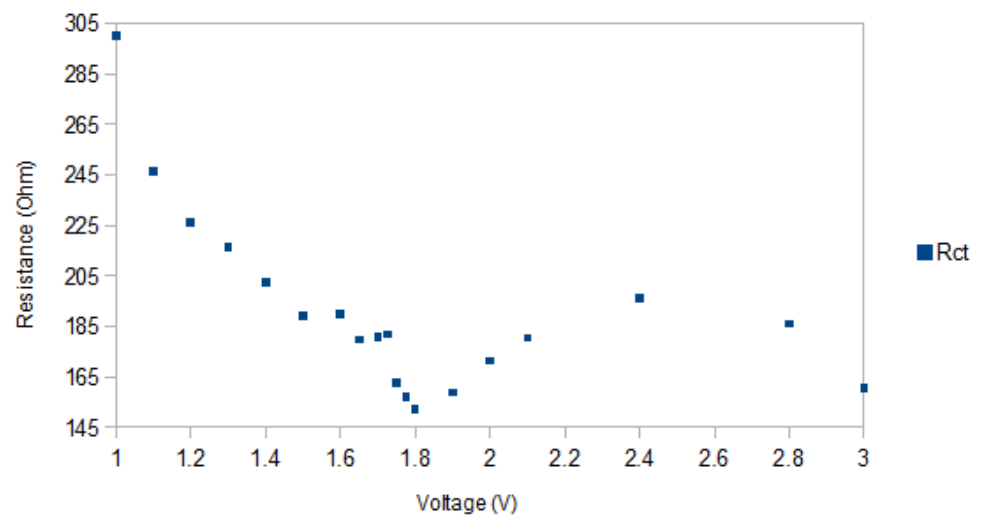

Figure D.9: Higher resolution version of Figure 4.4c, charge transfer resistance as a function of voltage.

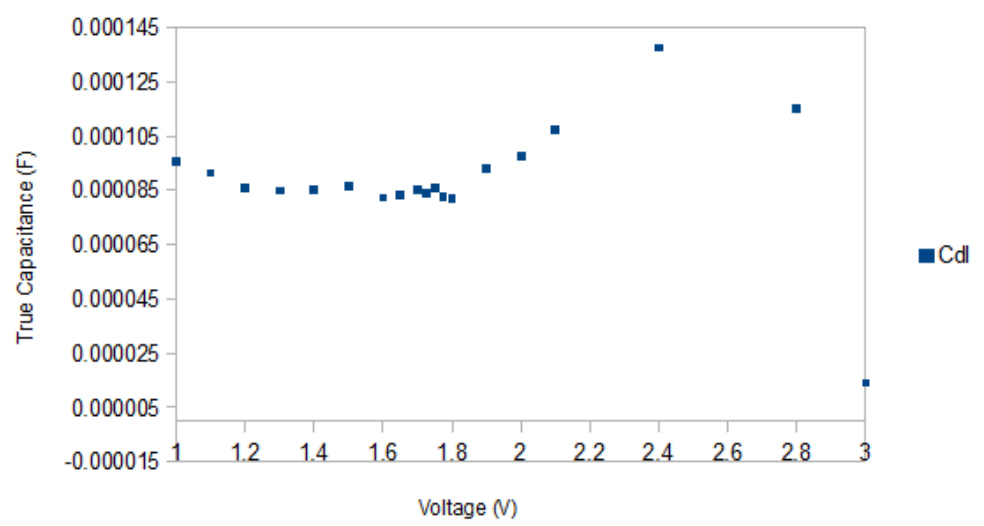

Figure D.10: Higher resolution version of Figure 4.4d, double layer capacitance as a function of voltage. 


\section{Appendix E}

\section{Supplementary Information for On the Complex Interplay of Crystallinity and Surface Area on Li-Ion Diffusion and Pseudocapacitive Storage Properties of Nanocrystalline Anatase}

This section provides supporting information for the paper whose title is above. Figure E.1 shows the individual X-ray diffraction (XRD) patterns for A0, A200, A300, and A400. This allows for the decrease in the size of the amorphous hump to be seen as the annealing temperature increases. Figure E. 2 shows the low rate $\left(1 \mathrm{C} ; \mathrm{C}=168 \mathrm{~mA} \mathrm{~g}^{-1}\right)$ cycling data for A0 over 100 cycles. Figure E. 3 is a plot of the capacity difference between A0 and A300 at various cycling rates. The fact that A0-A300 becomes positive at rates above $4 \mathrm{C}$ represents a shift in the major mechanism (from diffusion-based to surface area controlled) for insertion and extraction of lithium. Figure E.4 is the equivalent circuit model used with ZView software for fitting the electrochemical impedance spectroscopy data. $R_{e}$ is the electrolyte resistance, $R_{1}$ and $C_{1}$ form a parallel element representative of the electrode surface roughness and the inhomogeneity of the lithiation of the active material. $R_{c t}$ is the charge-transfer resistance, $C_{d l}$ is the double layer capacitance and $Z_{W}$ is the Warburg impedance. $R_{c t}, C_{d l}$ and $Z_{W}$ form a Randall's circuit that explains the direct interaction between lithium ions and the active material. Table E.1 gives the important data associated with the EIS spectra shown in Figure 5 in the paper. Figure E.5 shows graphically the trend in the values associated with the circuit elements presented in Table E.1. Figure E.6 shows important plots used to calculate $D_{L} i$. Figure E.6a plots open circuit voltage (OCV) vs. $x$ in $\mathrm{Li}_{\mathrm{x}} \mathrm{TiO}_{2}$ used 
to calculate $d E / d x$. Figures E.6b and E.6c plot $Z$ " vs $\omega^{-1 / 2}$ at various points during the lithathiation of $\mathrm{A} 0$ and $\mathrm{A} 300$, respectively, to determine the pre-exponential factor, $A$. Table E. 2 summarizes the calculated parameters determined from the plots in Figure E.6 as well as gives the diffusion coefficients for A0 and A300 at various states of lithiation. Figure E.7 shows a plot of $x$ in $\mathrm{Li}_{\mathrm{x}} \mathrm{TiO}_{2}$ vs. $D_{L} i$ for $\mathrm{A} 0$ and A300. Figure E. 8 gives the differential voltage profiles for $\mathrm{A} 0$ and A300, highlighting the changes in profile shape between the 1st and 500th cycle. Figure E.9 gives a comparisson of the XRD patterns of pristine electrodes and electrodes cycled 500 times for both A0 and A300. Minor peak shift is marked on the patterns with solid black lines. Figure E. 10 shows cycling data at $2 \mathrm{C}$ for $\mathrm{A} 0$ over 500 cycles showing only minor capacity loss. 

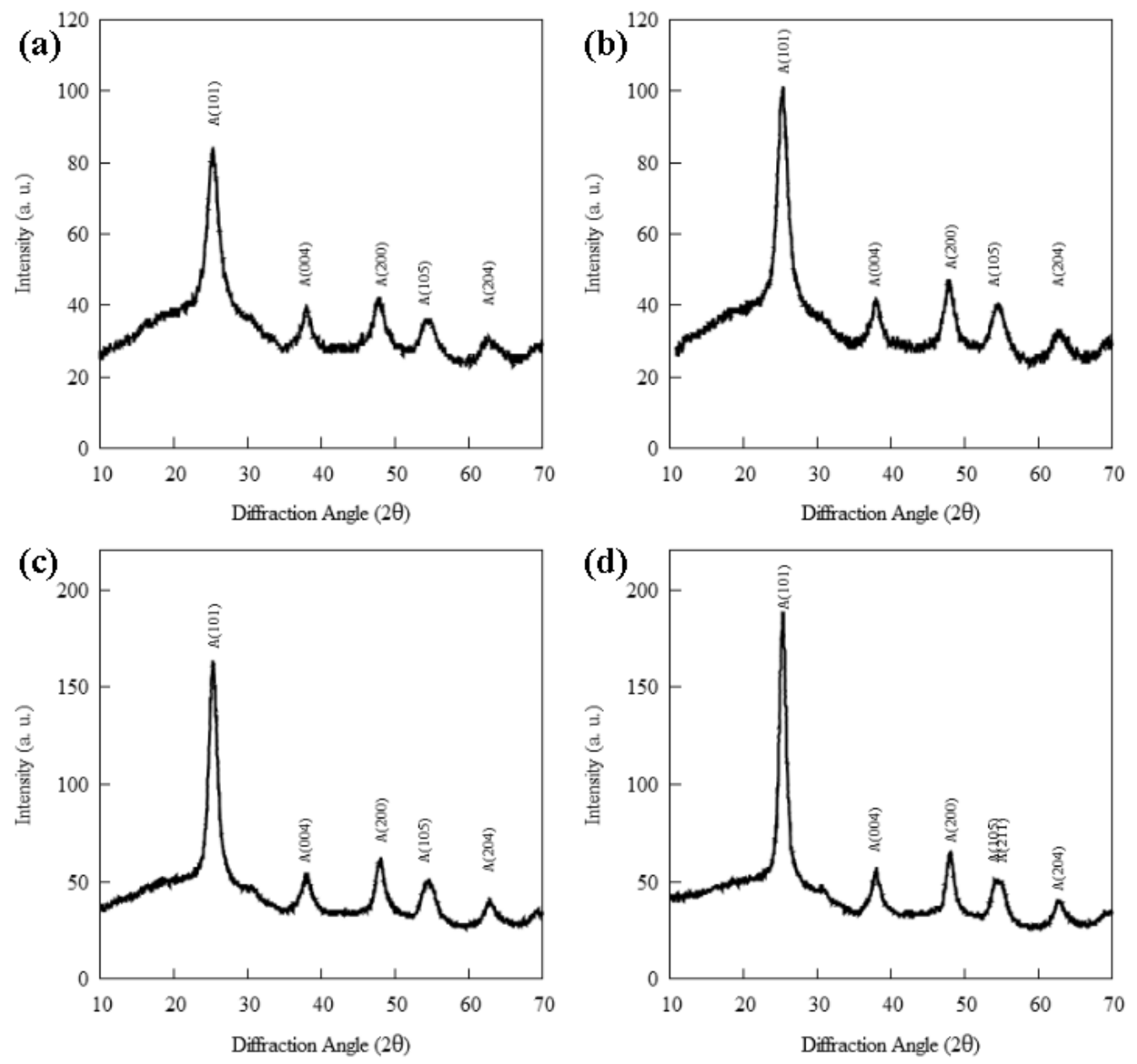

Figure E.1: Individual X-ray diffraction patterns for A0 (a), A200 (b), A300 (c) and A400 (d). The patterns show the decrease in amorphous hump due to increased crystallinity. 


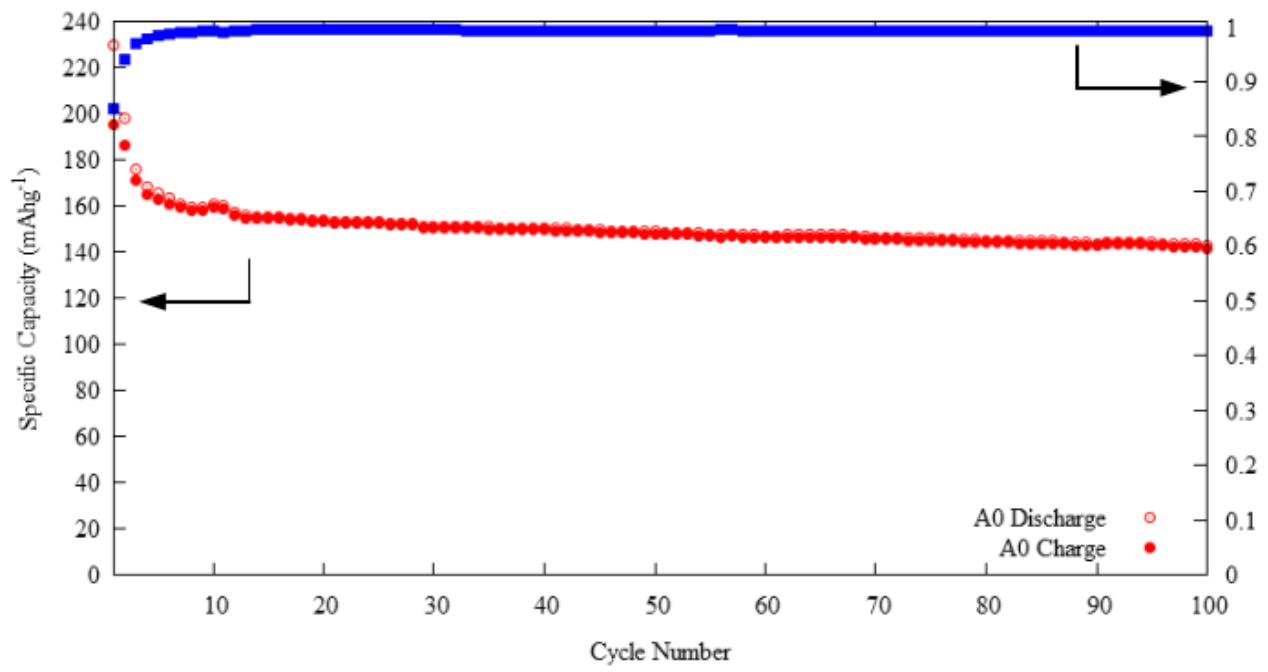

Figure E.2: Specific capacity and coulombic efficiency data for as-synthesized anatase (A0) cycled 100 times at $1 \mathrm{C}$ rate $\left(\mathrm{C}=168 \mathrm{~mA} \mathrm{~g}^{-1}\right)$.

Table E.1: Table of the important values at each point during the lithation for A0 and A300, determined by fitting the equivalent circuit to the Nyquist plots.

\begin{tabular}{ccccccc}
\hline Lithiation Point & $R_{e}(\Omega)$ & $R_{1}(\Omega)$ & $C_{1}(\mathrm{~F})$ & $R_{c t}(\Omega)$ & $C_{d l}(F)$ & Phase Angle $(\theta)$ \\
\hline A0: $0 \%$ & 3.709 & 16.31 & $7.55 \mathrm{E}-005$ & 20.39 & $3.62 \mathrm{E}-005$ & 79.72 \\
A0: $25 \%$ & 2.998 & 67.66 & $1.55 \mathrm{E}-004$ & 25.27 & $1.59 \mathrm{E}-005$ & 39.79 \\
A0: $50 \%$ & 1.629 & 48.5 & $4.94 \mathrm{E}-003$ & 13 & $1.99 \mathrm{E}-005$ & 53.52 \\
A0: $75 \%$ & 2.513 & 12.58 & $9.94 \mathrm{E}-005$ & 117.9 & $6.36 \mathrm{E}-005$ & 63.12 \\
A0: $100 \%$ & 4.181 & 11.66 & $2.19 \mathrm{E}-005$ & 124.5 & $5.68 \mathrm{E}-005$ & 67.04 \\
A300: $0 \%$ & 2.671 & 8.209 & $6.50 \mathrm{E}-004$ & 13.17 & $4.09 \mathrm{E}-005$ & 78.95 \\
A300: $25 \%$ & 5.605 & 24.74 & $1.44 \mathrm{E}-004$ & 5.613 & $6.15 \mathrm{E}-006$ & 46.48 \\
A300: $50 \%$ & 2.423 & 6.376 & $1.42 \mathrm{E}-004$ & 9.804 & $1.33 \mathrm{E}-005$ & 60.37 \\
A300: $75 \%$ & 2.877 & 4.691 & $8.68 \mathrm{E}-005$ & 15.88 & $8.54 \mathrm{E}-005$ & 66.18 \\
A300: $100 \%$ & 3.009 & 4.789 & $5.91 \mathrm{E}-005$ & 16.42 & $1.89 \mathrm{E}-004$ & 68.32 \\
\hline
\end{tabular}




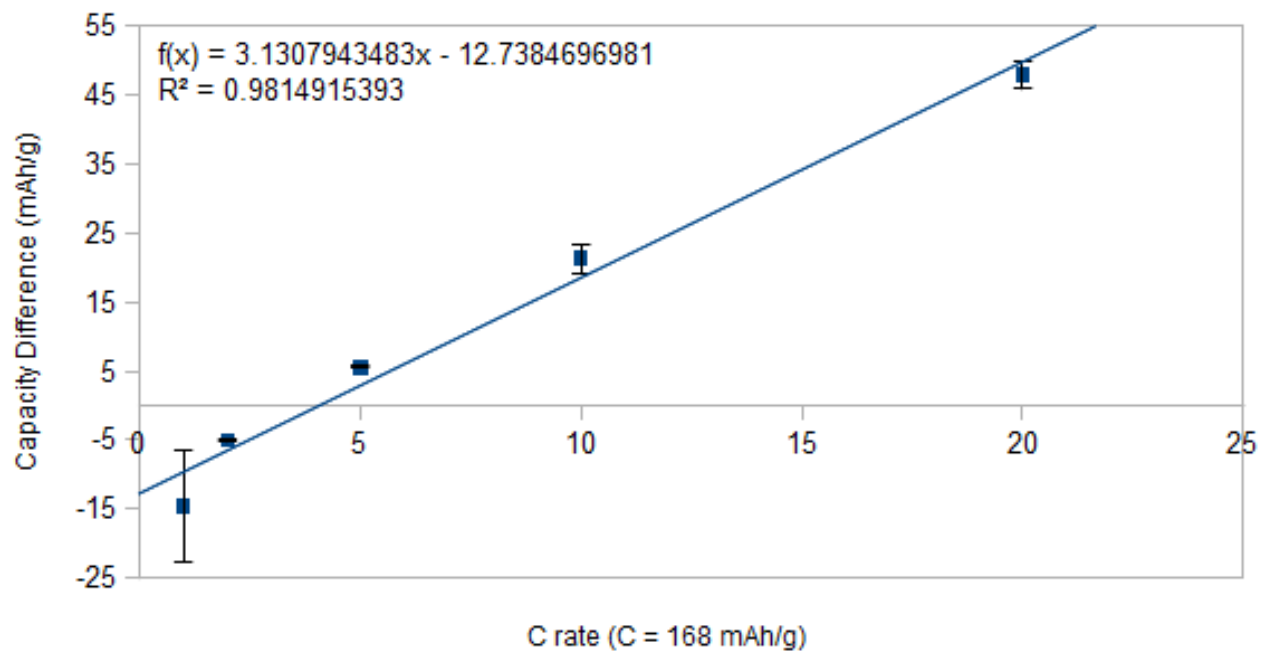

Figure E.3: Plot of reversible capacity difference between $\mathrm{A} 0$ and $\mathrm{A} 300$ at various rates. At $\sim 4 \mathrm{C}$ the capacity should be the same indicating a balance between diffusion-based and surface area based as the major charge storage method.

Table E.2: Summary of diffusion coefficient parameters.

\begin{tabular}{cccc}
\hline $\begin{array}{c}\text { Lithiation } \\
\text { Point }\end{array}$ & $x$ in $\mathrm{Li}_{\mathrm{x}} \mathrm{TiO}_{2}$ & $\begin{array}{c}\text { Pre-exponential } \\
\text { Factor }(A)\end{array}$ & $\begin{array}{c}\text { Diffusion } \\
\text { Coefficient }\left(\mathrm{cm}^{2} \mathrm{~g}^{-1}\right)\end{array}$ \\
\hline A0: $0 \%$ & 0 & 29.024 & $1.11 \mathrm{E}-016$ \\
$\mathrm{~A} 0: 25 \%$ & 0.127 & 4.9295 & $3.84 \mathrm{E}-015$ \\
$\mathrm{~A} 0: 50 \%$ & 0.245 & 7.75 & $1.55 \mathrm{E}-015$ \\
$\mathrm{~A} 0: 75 \%$ & 0.371 & 10.723 & $8.12 \mathrm{E}-016$ \\
$\mathrm{~A} 0: 100 \%$ & 0.484 & 18.415 & $2.75 \mathrm{E}-016$ \\
$\mathrm{~A} 300: 0 \%$ & 0 & 29.111 & $2.33 \mathrm{E}-016$ \\
$\mathrm{~A} 300: 25 \%$ & 0.127 & 3.807 & $1.36 \mathrm{E}-014$ \\
$\mathrm{~A} 300: 50 \%$ & 0.229 & 9.5284 & $2.17 \mathrm{E}-015$ \\
$\mathrm{~A} 300: 75 \%$ & 0.387 & 13.312 & $1.11 \mathrm{E}-015$ \\
A300: $100 \%$ & 0.5 & 13.328 & $1.11 \mathrm{E}-015$ \\
\hline
\end{tabular}




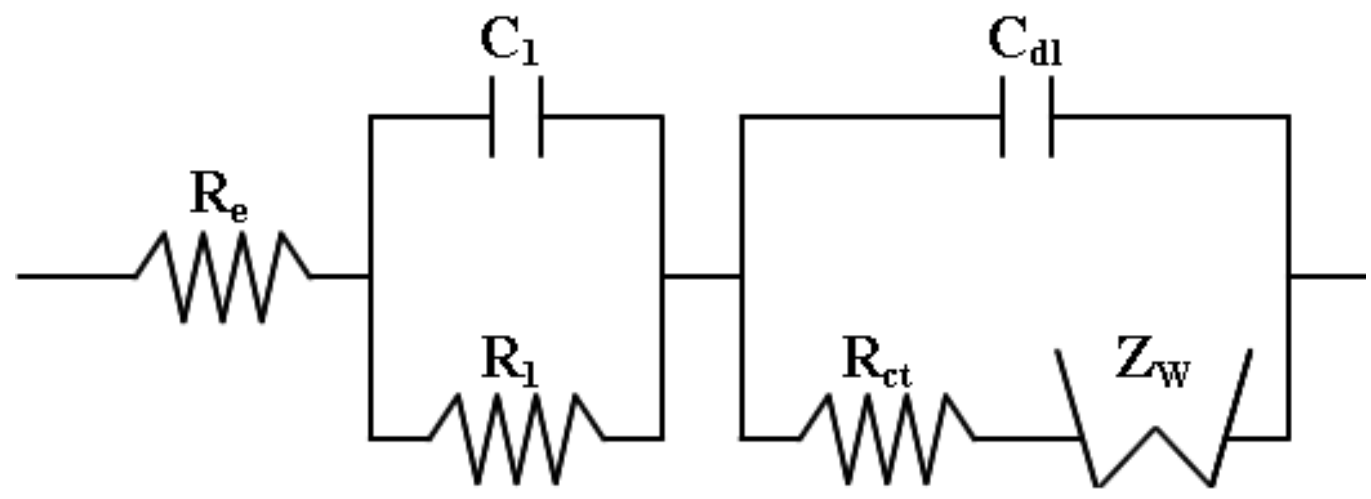

Figure E.4: Equivalent circuit model for fitting the electrochemical impedance spectroscopy data. $R_{e}$ is the electrolyte resistance, $R_{1}$ and $C_{1}$ form a parallel element representative of the electrode surface roughness and the inhomogeneity of the lithiation of the active material. $R_{c t}$ is the charge-transfer resistance, $C_{d l}$ is the double layer capacitance and $Z_{W}$ is the Warburg impedance. $R_{c t}, C_{d l}$ and $Z_{W}$ form a Randall's circuit that explains the direct interaction between lithium ions and the active material. 

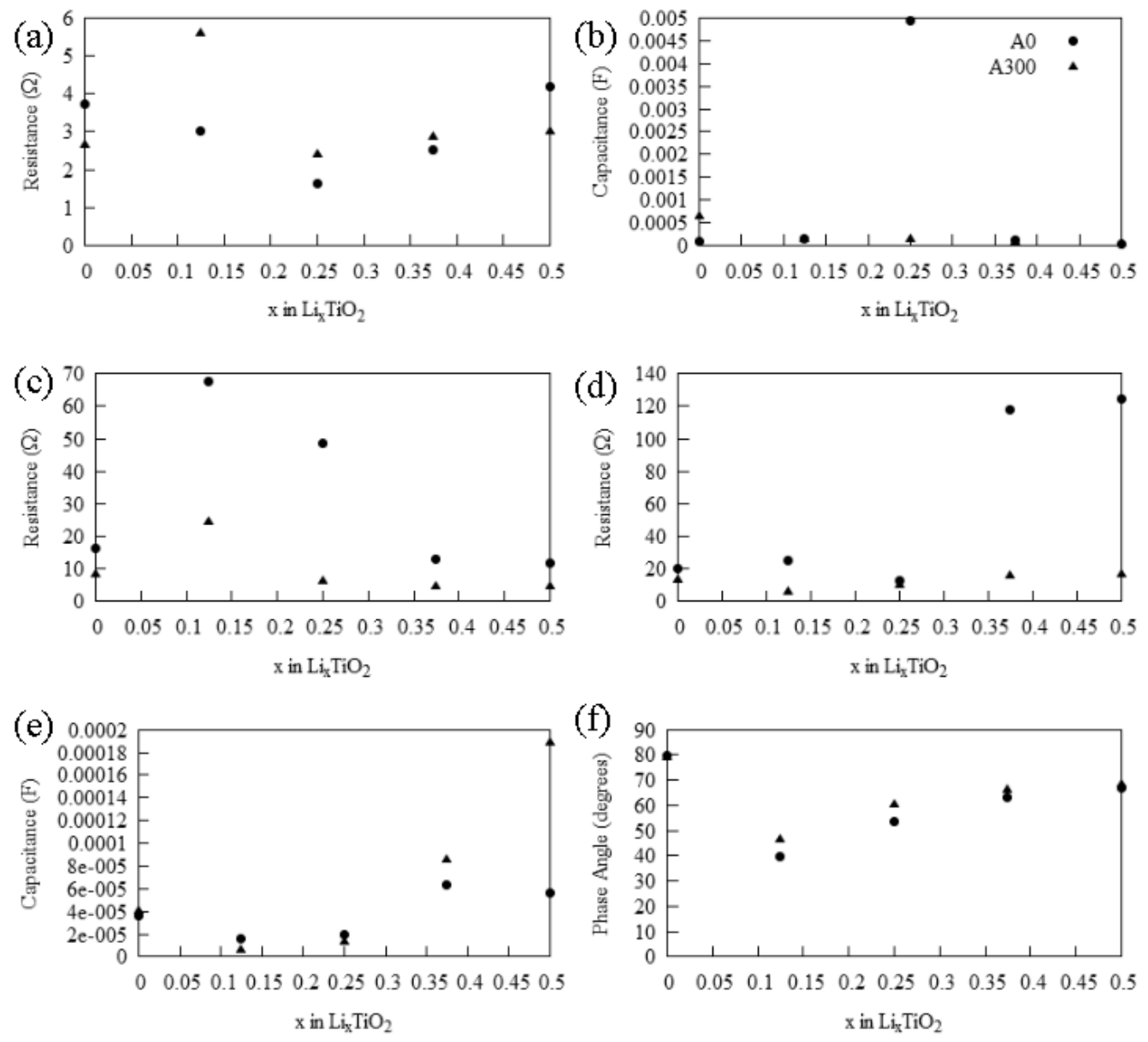

Figure E.5: Plots showing the trend of $R_{e}(\mathrm{a}), C_{1}(\mathrm{~b}), R_{1}(\mathrm{c}), R_{c t}$ (d), $C_{d l}$ (e), and phase angle (f) at various points during lithiation. 

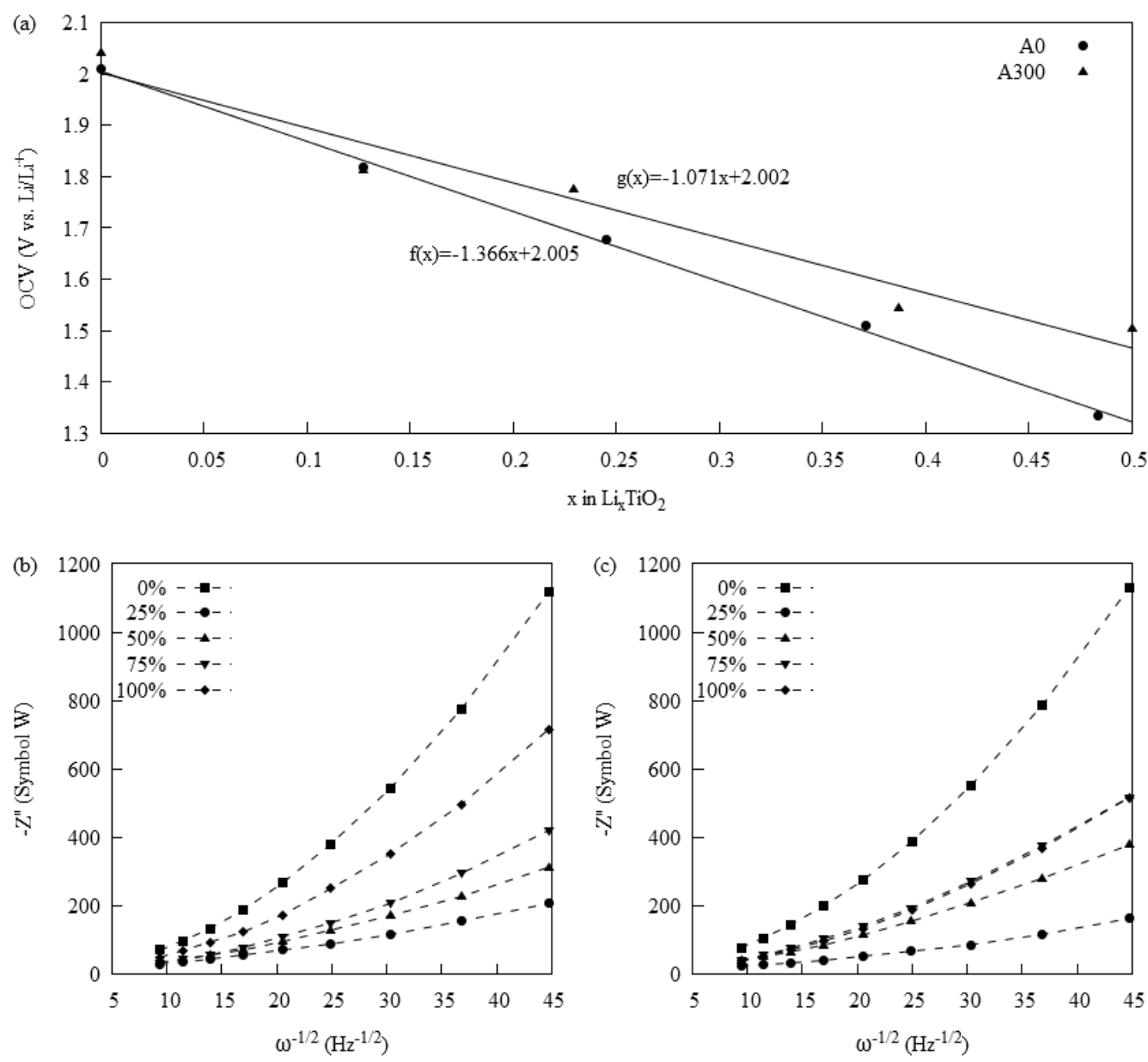

Figure E.6: Plots of OCV vs. $x$ in $\mathrm{Li}_{\mathrm{x}} \mathrm{TiO}_{2}$ used to calculate $d E / d x$ (a) and Z" vs $\omega^{-1 / 2}$ at various points during the lithathiation of $\mathrm{A} 0$ (b) and $\mathrm{A} 300$ (c) used to determine the pre-exponential factor, $A$, used in the calaculation of $D_{L} i$. 


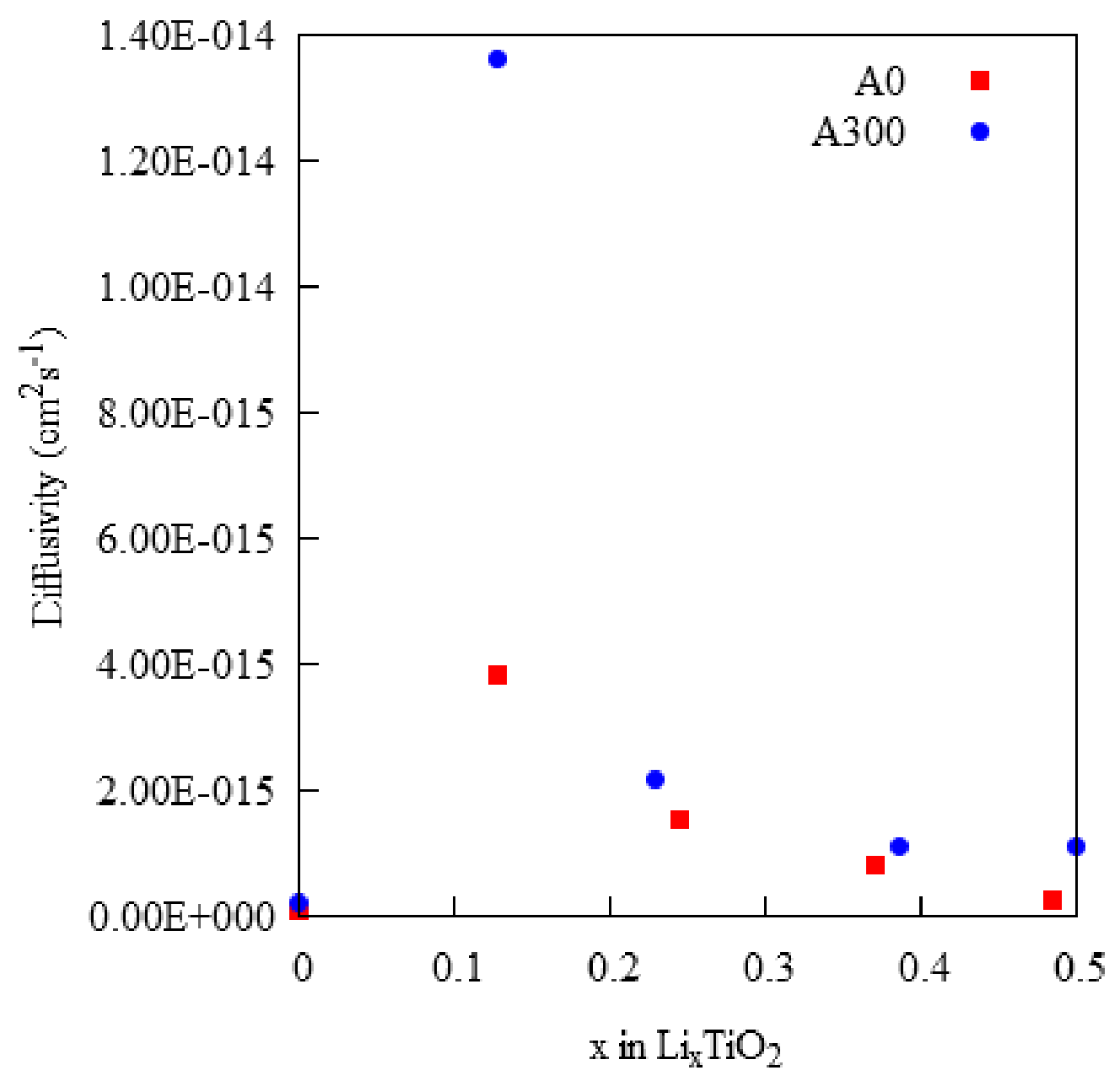

Figure E.7: Plot comparing the diffusion coefficients of A0 and A300 at various lithiation states. 

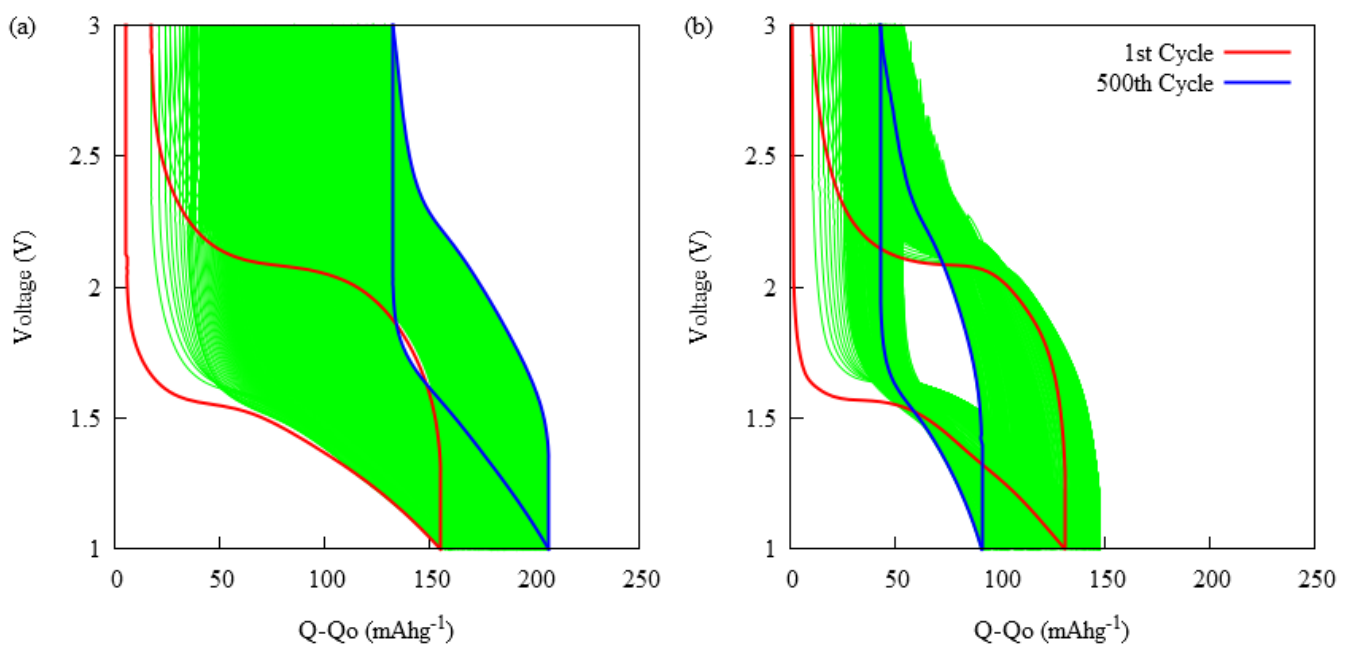

Figure E.8: Differential voltage profiles for A0 (a) and A300 (b) highlighting the changes in profile shape between the 1 st and 500th cycle. 

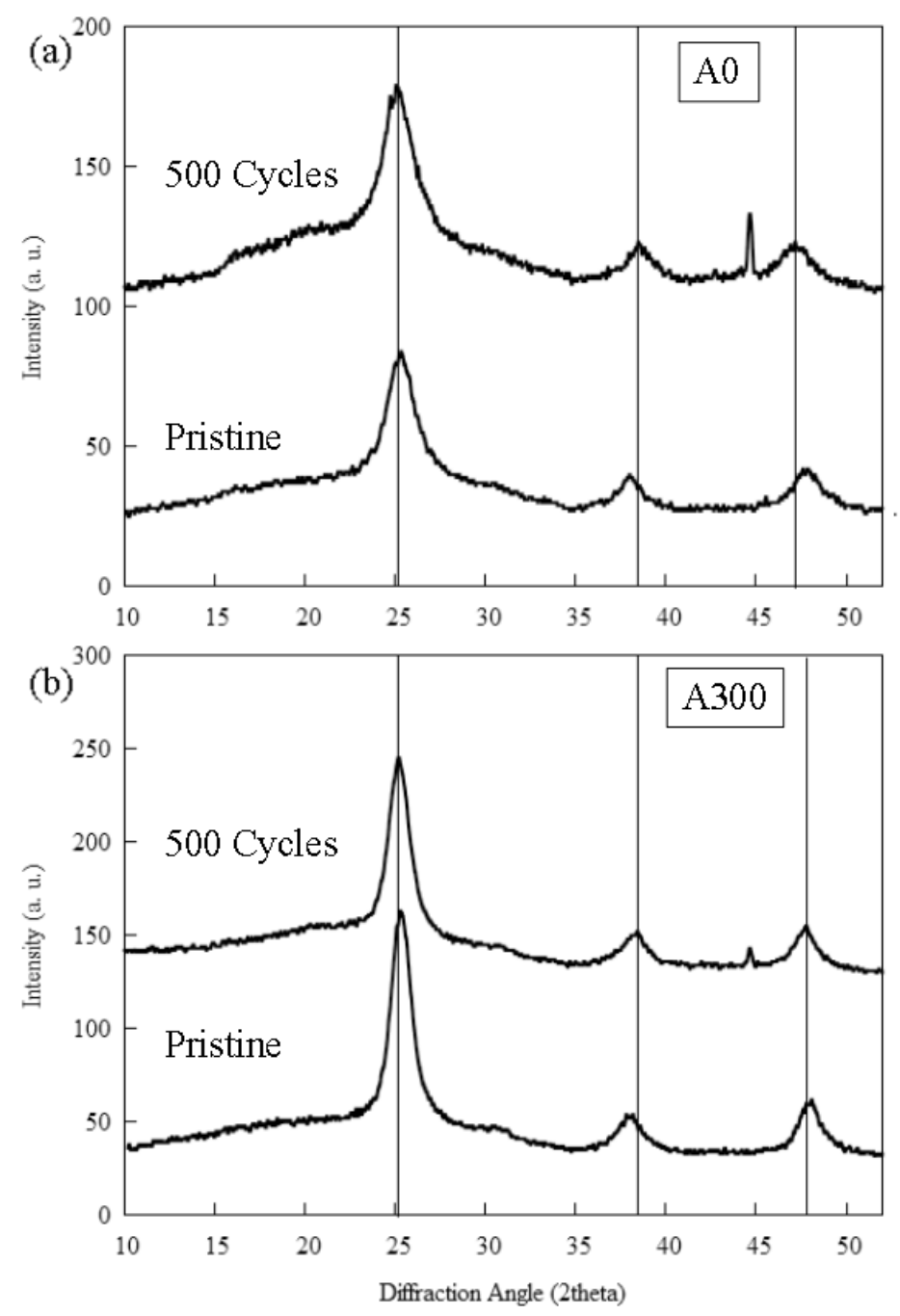

Figure E.9: XRD patterns for $\mathrm{A} 0$ (a) and $\mathrm{A} 300$ (b) comparing the pristine electrode and the electrode after 500 cycles at $10 \mathrm{C}$. Peak shift is marked by lines through the pattern for the electrode after 500 cycles. 


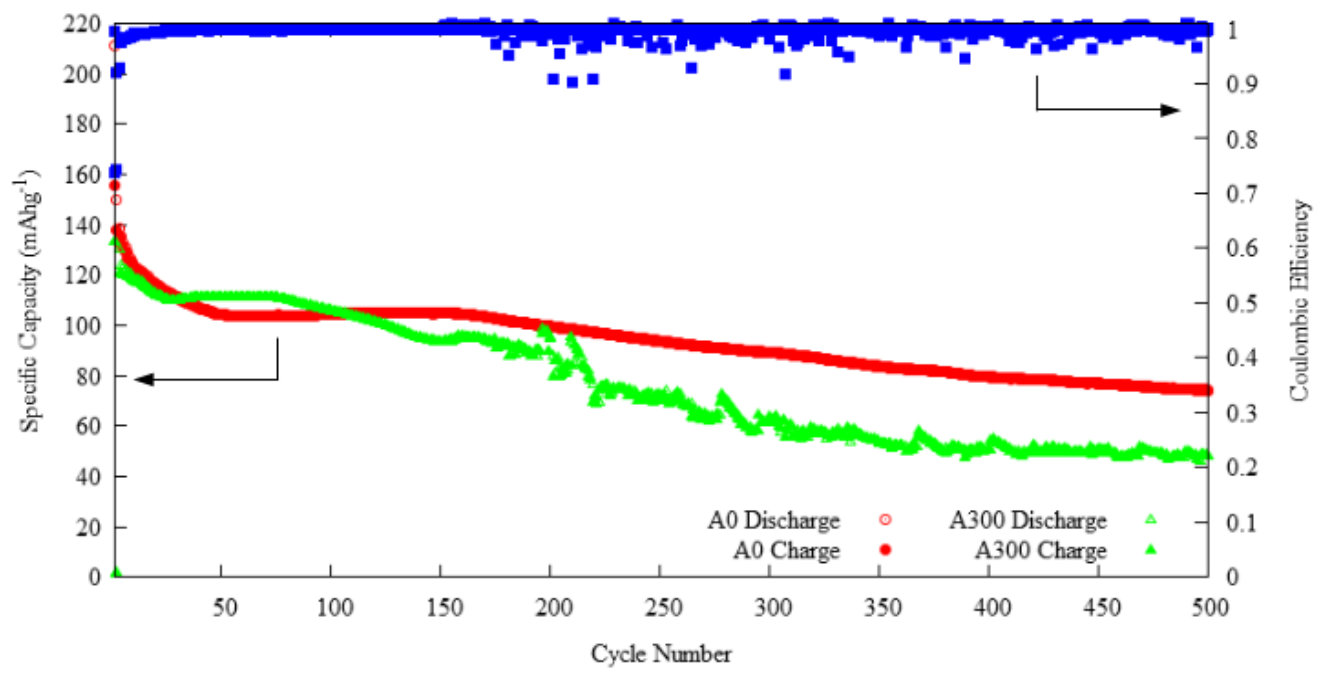

Figure E.10: Specific capacity and coulombic efficiency data for as-synthesized anatase (A0) cycled 500 times at $2 \mathrm{C}$ rate $\left(\mathrm{C}=168 \mathrm{~mA} \mathrm{~g}^{-1}\right)$. 


\section{Appendix F}

\section{Supplementary Information for Engineering 3-D \\ Li-Ion Electrodes with Enhanced Charge Storage Properties based on Solution-Processed and Sintered Anatase Nanocrystal-Carbon Mesoporous Structures}

This section provides supporting information for the paper whose title is presented above. The supporting information comprises supplementary material and electrochemical characterization data; discussion of the effect of different sintering parameters; and details concerning the various experimental procedures used for synthesis, fabrication, SEM and image analysis.

\section{F.1 Supplementary Characterization Data}

Figure F.1 provides a transmission electron micrograph of the nanoparticles used in this work. The aqueous synthesized anatase nanoparticles were previously characterized for size and surface area [1]. It was found that they were $\sim 6 \mathrm{~nm}$ in diameter with $222 \mathrm{~m}^{2} \mathrm{~g}^{-1}$ surface area. The crystallites had an isotherm (Figure reffig:3Disotherm) that is indicative of a non-porous nanosolids. After fabrication, the standard and 3-D electrode films were investigated. The shape of the isotherms of both films is the same, and is indicative of mesoporous materials. The lower adsorption and desorption as compared to the particulate anatase indicates a lower 
surface area. During the sintering process (applicable to 3-D electrodes), interparticle necking occurs as well as some particle coarsening associated with the elevated temperature required for sintering. However, the standard films are not exposed to high temperature; this implies that the observed reduced surface area is not due crystallite coarsening but rather postulated to be associated with binder coating of particles. In order to adhere particles together, the binder must partially coat the particles. This is explained by the difference in pore size between the two electrodes.

Figure F.3 shows the pore size distribution for the two electrode films. The standard film has an average pore size of $7.6 \mathrm{~nm}$, making it mesoporous, with 25 $\mathrm{nm}$ macropores formed due to aggregation of nanoparticles. The 3-D electrode has an average pore size of $15 \mathrm{~nm}$, but as the figure shows, there are maxima at 22,17 , and $10 \mathrm{~nm}$. The dispersants added to the paste formulation, as well as ultrasonication, help to limit the aggregation of nanocrystallites into large secondary particles. While aggregation does occur, the limited size allows for an hierarchy of porosity. Pore formation is facilitated in the 3-D electrode via volatilization of the organic paste components, namely $\alpha$-terpineol and ethyl cellulose. Electrolyte infiltration and Li-ion shuttling is easier with the 3-D electrode pore distribution. 


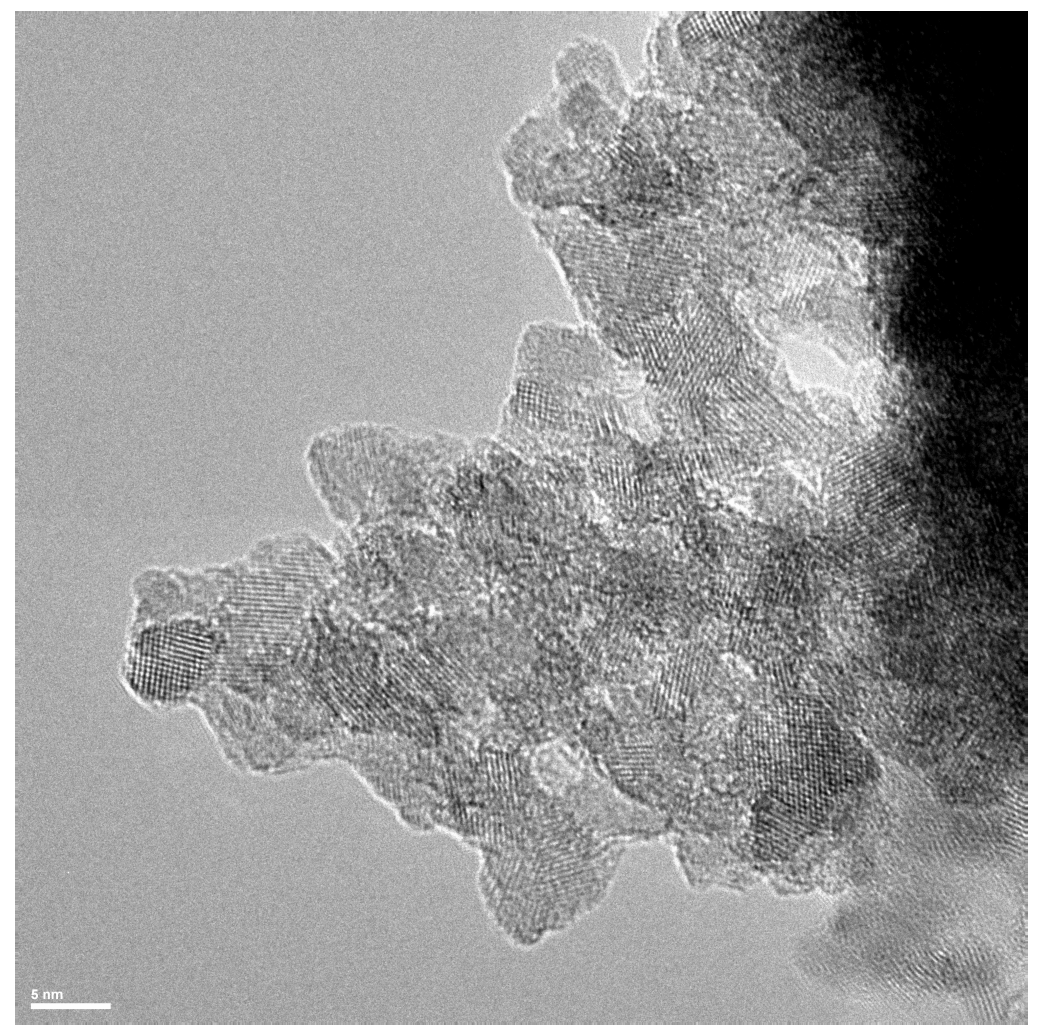

Figure F.1: Transmission electron micrograph of the as-prepared anatase crystals. Micrograph shows a non-porous nanospherical morphology, and an average crystallite size of approximately $6 \mathrm{~nm}$ 


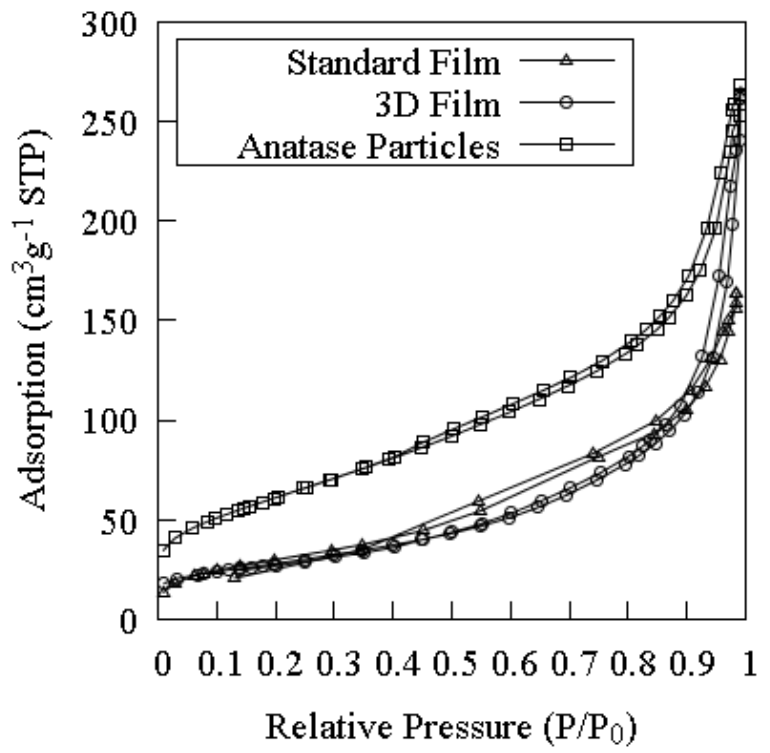

Figure F.2: Pore size distribution for standard and 3-D electrode films. 


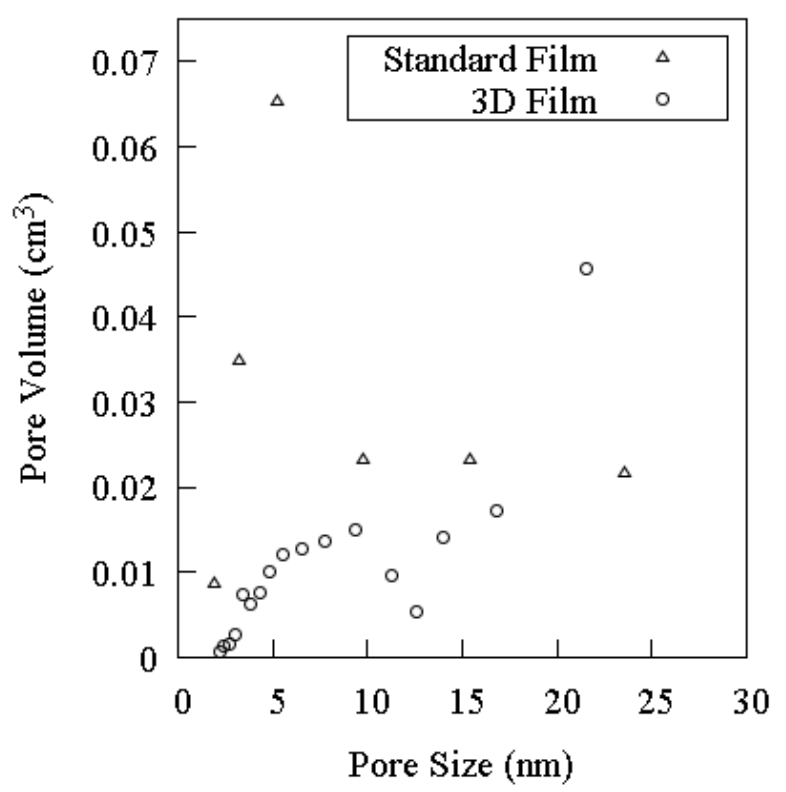

Figure F.3: $\mathrm{N}_{2}$ Isotherms for anatase nanocrystallites, standard electrode, and 3-D electrode films. 
The isotherms in Figure F.2 show that the films have a lower surface area compared to the crystallites. As previously mentioned, the anatase nanoparticles have a surface area of $222 \mathrm{~m}^{2} \mathrm{~g}^{-1}$. Both the standard and 3-D films, however, have a surface area of $\sim 100 \mathrm{~m}^{2} \mathrm{~g}^{-1}$ (Table F.1). As PVDF (representing 10\% of the total mass) thickens the particles via a loss in surface area occurs. For the 3-D electrode this loss is explained by the coarsening of particles during the sintering step.

Figure F.4 shows a high magnification scanning electron micrograph of the structure of the 3-D electrode coupled with schematics of the standard and 3-D electrode structures. This micrograph highlights the mesoporous structure as well as inter-particle necking caused by sintering, while the schematics provide a simple illustration of the differences in electrode structures. Figure F.5 shows the initial voltage profiles of both the standard and 3-D electrodes at C/5 rate. Figure F.6 shows the high rate (10C) cycling of the standard and 3-D electrodes over 100 cycles. 

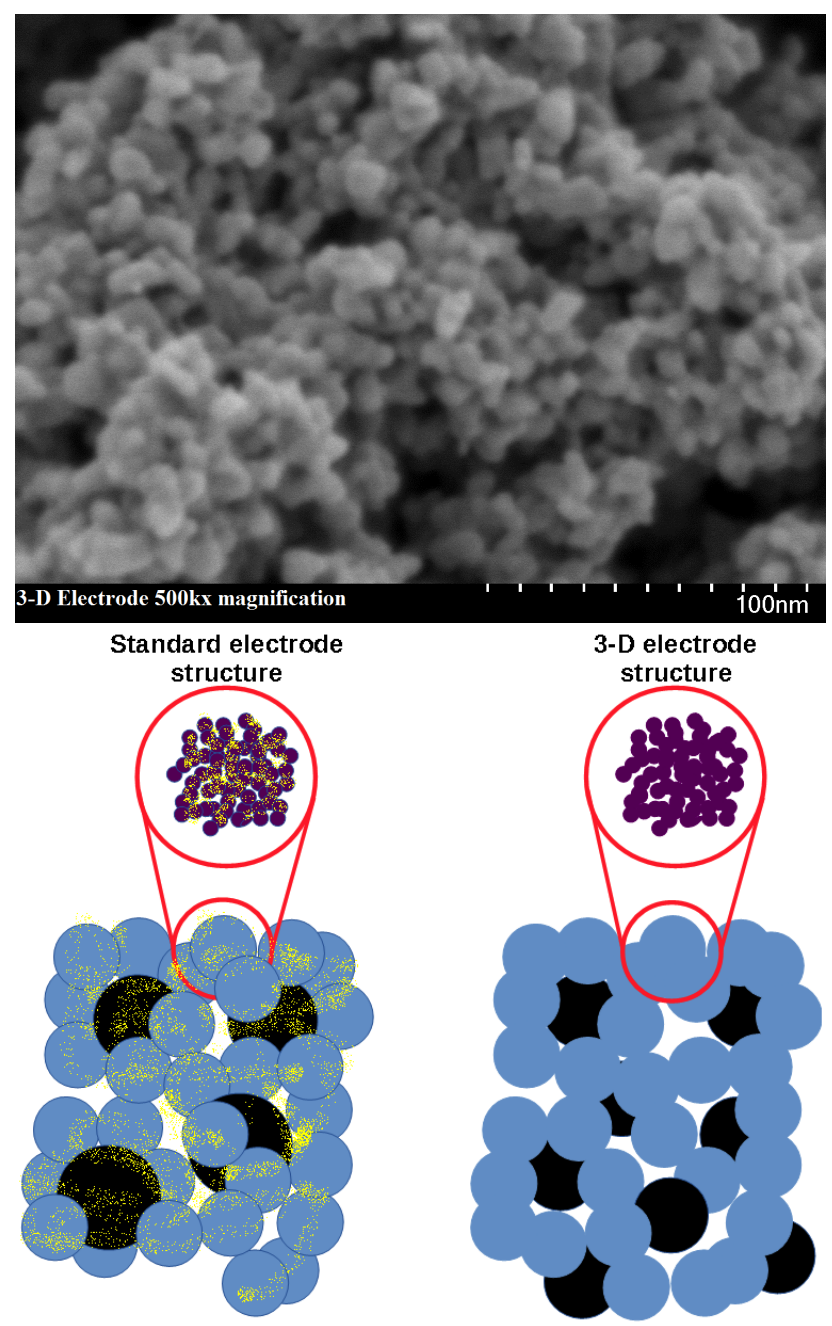

Figure F.4: High magnification secondary electron micrograph of the 3-D electrode structure (top) coupled with schematics of the standard and 3-D electrode structures to highlight differences. Standard electrodes have individual nanoparticles (purple) forming a larger secondary particle (blue). These secondary particles contact each other and large carbon clusters (black) at single point and are held together by PVDF binder (yellow). The 3-D electrodes have nanoparticles that neck to form a rod-like structure which builds the secondary particles. These particles also neck to each other and contact well dispersed carbon particles at multiple places within the structure. 


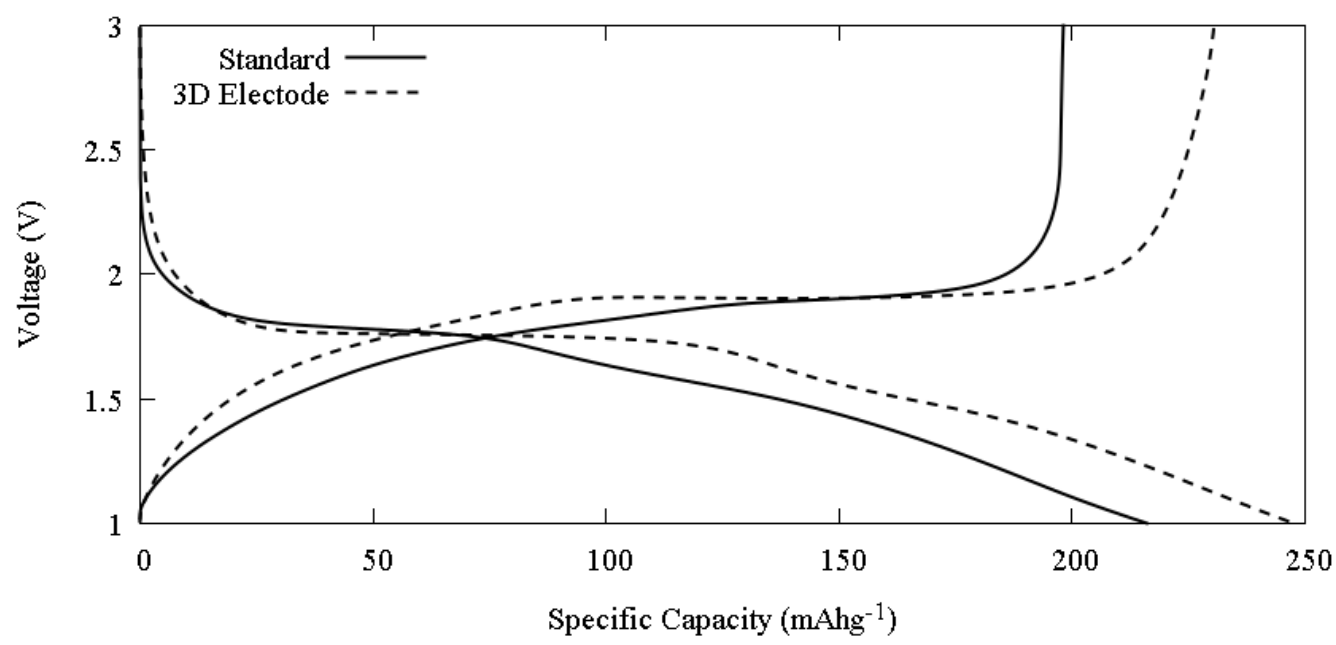

Figure F.5: Voltage-capacity profiles for standard (solid line) and 3-D (dashed line) electrodes during the initial lithiation and delithiation cycle at $\mathrm{C} / 5$ rate

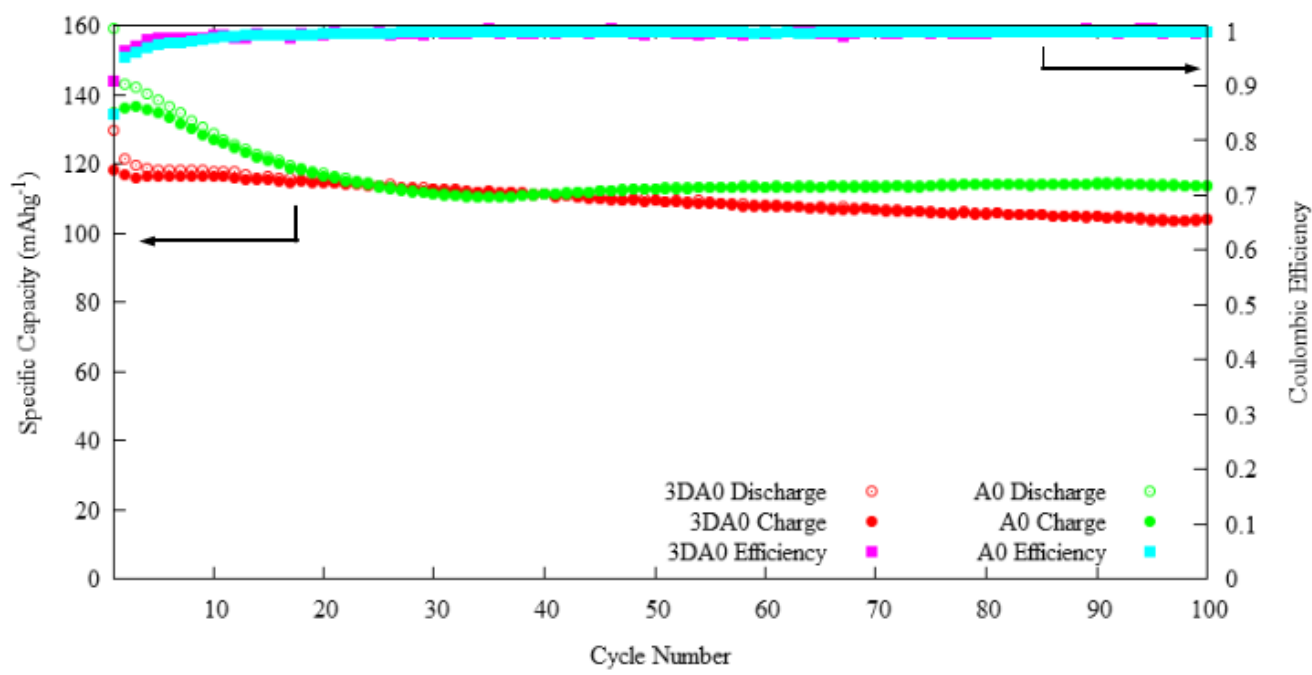

Figure F.6: Galvanostatic charge/discharge over 100 cycles between cutoff voltages of $1 \mathrm{~V}$ (discharge) and $3 \mathrm{~V}$ (charge) at $10 \mathrm{C}$ rate $\left(1680 \mathrm{~mA} \mathrm{~g}^{-1}\right)$ 
Table F.1: Key properties of active material and electrode films based on BET surface area analysis.

\begin{tabular}{ccccc}
\hline Sample & $\begin{array}{c}\text { Surface Area } \\
\left(\mathrm{m}^{2} \mathrm{~g}^{-1}\right)\end{array}$ & $\begin{array}{c}\text { Crystallite } \\
\text { Size }(\mathrm{nm})\end{array}$ & $\begin{array}{c}\text { Average Pore } \\
\text { Width }(\mathrm{nm})\end{array}$ & $\begin{array}{c}\text { Percent } \\
\text { Porosity (\%) }\end{array}$ \\
\hline Active material (anatase) & 222 & 6.23 & $\diamond$ & $\diamond$ \\
Standard electrode & 102 & $\diamond$ & 7.6 & 54 \\
3-D electrode & 100 & $\diamond$ & 15 & 52 \\
\hline
\end{tabular}

\section{F.2 Sintering Effects}

The 3-D electrodes are fabricated using a method adapted from the dye-sensitized solar cell field $[2,3]$. The method consists of making a paste for doctor blading or screen printing followed by annealing/sintering. For paste making $\mathrm{TiO}_{2}$ and acetylene black are dispersed in anhydrous ethanol. As a rheologic agent (and to provide dispersion for $\mathrm{TiO}_{2}$ ), $\alpha$-terpineol is added to the solvent, then ethyl cellulose is added to improve the consistency of the paste as well as help build the porous structure of the electrode upon volatilization during the sintering step. The ethanolic solution is evaporated to the desired viscosity using a rotary evaporator (operated at $45^{\circ} \mathrm{C}$ and $0.26 \mathrm{~atm}$ ), applied to $\mathrm{Al}$ substrate by doctor blading and dried overnight at $80^{\circ} \mathrm{C}$. The resulting film is then sintered using the sintering profile seen in Figure F.7 [2]. The first step in the process removes any moisture that has become trapped in the film. The second step ramps the temperature well above the boiling point $\left(219^{\circ} \mathrm{C}\right)$ for $\alpha$ - terpineol, before ramping a second time to burn out ethyl cellulose and begin particle inter-necking of $\mathrm{TiO}_{2}$. The final temperature step promotes crystal annealing.

Sintering causes the particles to neck and undergo crystal annealing, however, it also promotes particle coarsening that leads to a loss of surface area from $222 \mathrm{~m}^{2} \mathrm{~g}^{-1}$ associated with the active materials nanocrystals to $100 \mathrm{~m}^{2} \mathrm{~g}^{-1}$ for the sintered 3-D film (Table F.1). In the meantime due to volatilization of the organic components used to make the paste ( $\alpha$-terpineol and ethyl cellulose), the 3-D film has larger 


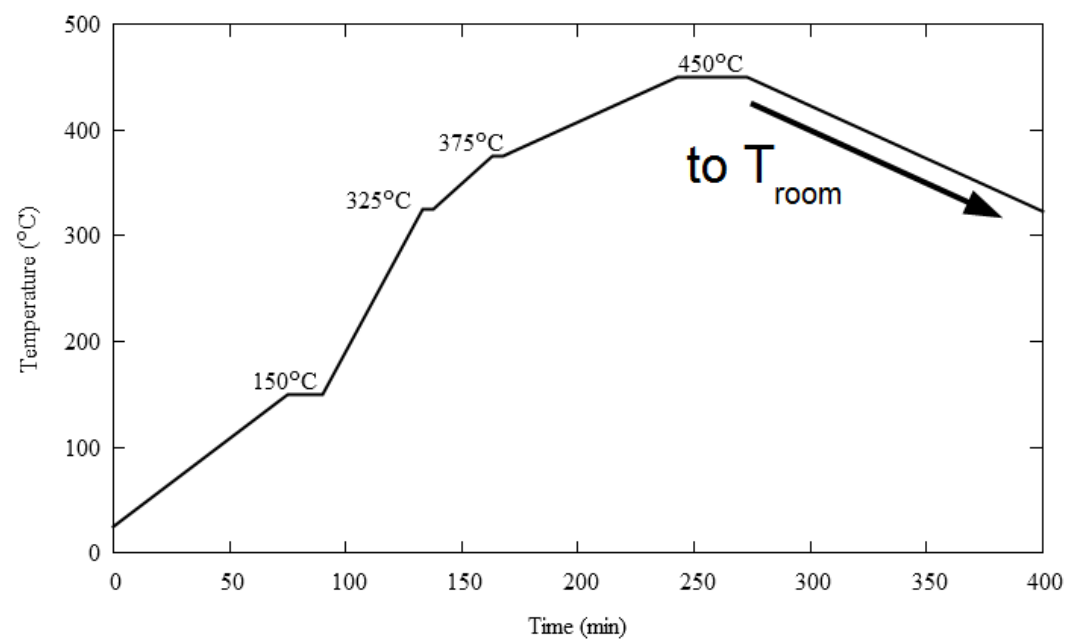

Figure F.7: Sintering profile for 3-D electrodes with temperature steps highlighted. Temperature returns to $25^{\circ} \mathrm{C}$ after 7 hours (not shown on profile)

average pore size $(15 \mathrm{~nm})$ than the PVDF-based standard film $(7.6 \mathrm{~nm}$ average pore size). Otherwise, because of particle aggregation, the binder-based electrode maintains the same overall porosity percentage at approximately $54 \%$ compared to $52 \%$ for the 3-D electrode.

The selected thermal profile for sintering is very important and needs to be optimized for different active materials. For one thing the temperature and time should be high/long enough to promote volatilization of the organic components (terpineol and ethyl cellulose) (at least $375^{\circ} \mathrm{C}$; [4]) responsible for creating the mesopores as well annealing of the crystals (at least $300^{\circ} \mathrm{C}$ [1]). On the other hand excessive temperatures and times during sintering should be avoided as they lead to loss of nanoscale properties (crystallite size and surface area) [1]. To demonstrate the importance of sintering temperature/time conditions we prepared two 3-D sintered electrodes, one following the profile of Figure F.7 and one following the profile of Figure F.8 featuring longer heating time at $400^{\circ} \mathrm{C}$. The temperature profile is similar to that of Figure F.7 until it reaches $325^{\circ} \mathrm{C}$, where it ramps directly 
to $400{ }^{\circ} \mathrm{C}$. This allows for the burnout/volatilization of $\alpha$-terpineol/ethyl cellulose, crystal annealing and inter-particle necking. The temperature then decreases to $25^{\circ} \mathrm{C}$ the same way as the original profile did.

Rate capability electrochemical testing was performed on the two sintered 3-D electrodes with the optimum sintering profile (Figure F.7) and the non-optimized sintering profile (Figure F.8). The results are shown in Figure F.9. At C/5 rate, the formation cycle of the $400{ }^{\circ} \mathrm{C}$ annealed sample (3D400) (Profile in Figure F.8) has an initial insertion capacity of $296.4 \mathrm{~mA} \mathrm{~h} \mathrm{~g}^{-1}$ which is the equivalent of $x=0.88$ in $\mathrm{Li}_{\mathrm{x}} \mathrm{TiO}_{2}$, almost the theoretical capacity of anatase $\left(335 \mathrm{~mA} \mathrm{~h}^{-1}\right)$. This is compared to the standard profile electrode (3D), which is a bit lower at $272.2 \mathrm{~mA} \mathrm{~h} \mathrm{~g}^{-1}$ $\left(x=0.81\right.$ in $\left.\mathrm{Li}_{\mathrm{x}} \mathrm{TiO}_{2}\right)$. However, the charge (delithiation) capacities for 3D400 and $3 \mathrm{D}$ are in the reverse order, i.e. 194 and $238 \mathrm{~mA} \mathrm{~h} \mathrm{~g}^{-1}$, respectively. That is the over-sintered electrode exhibits large polarization. As the electrodes cycle, even at low rate, there is sizable capacity loss in 3D400 that is not seen in 3D. The Coulombic efficiencies finally equalize at just under $100 \%$, however, at this point the capacity difference between the two electrodes is extremely high. This set of electrochemical data proves that sintering needs to be carefully conducted by balancing the requirements for inter-particle necking, crystal annealing and pore creation without excessive particle coarsening and surface area loss. 


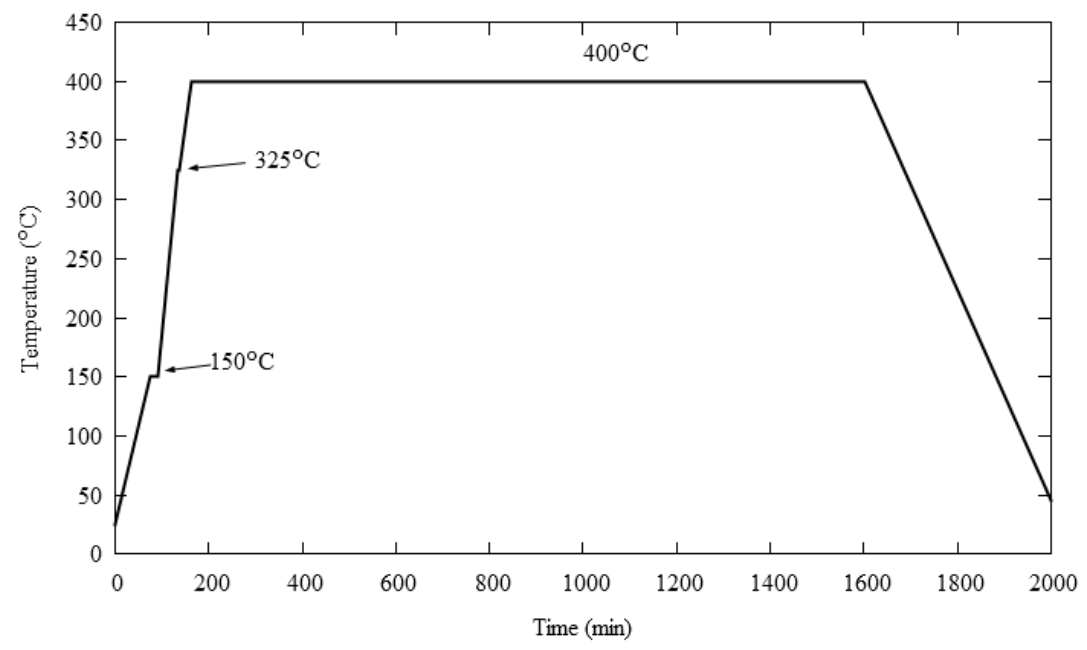

Figure F.8: Secondary sintering profile with lower necking temperature. Temperature is held for longer period of time before returning back to $25^{\circ} \mathrm{C}$

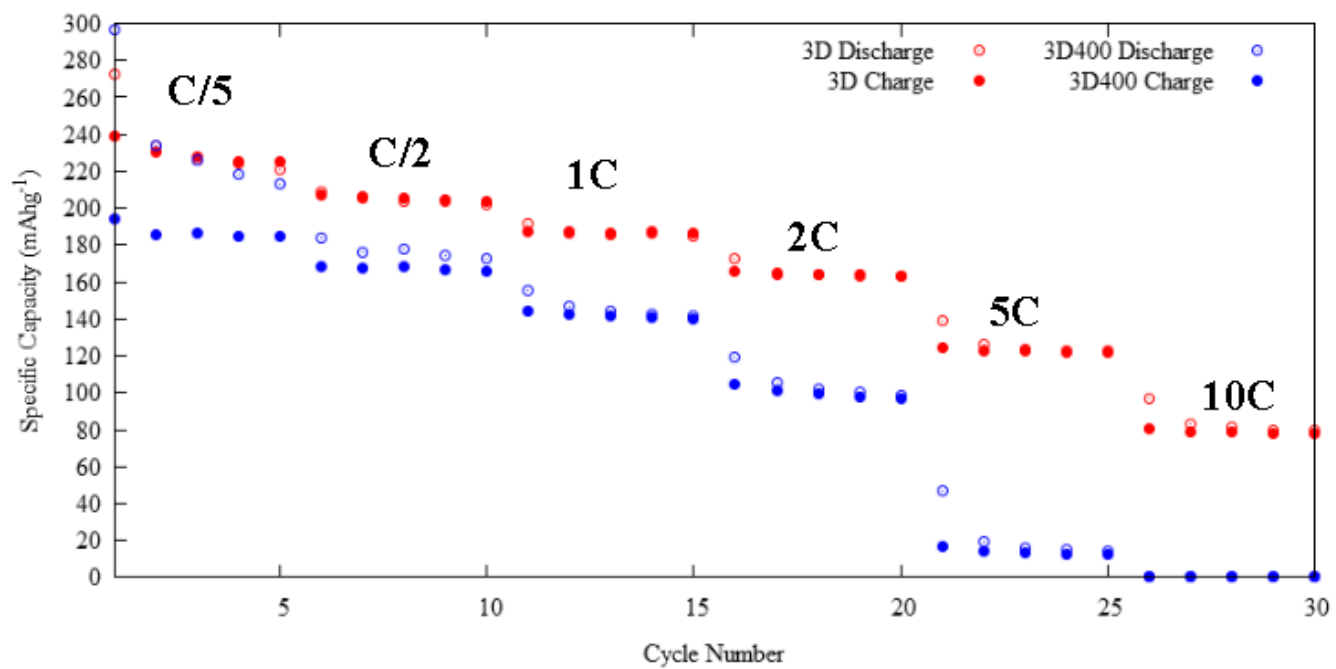

Figure F.9: Rate capability for 3D and 3D400 cycled between upper and lower cutoff voltages of 3 and $1 \mathrm{~V}$, respectively 


\section{F.3 Experimental Methods}

Aqueous synthesized anatase nanoparticles were used as the active material in all electrodes. Their synthesis was made via forced hydrolysis of an aqueous solution of $\mathrm{TiCl}_{4}$ at $80^{\circ} \mathrm{C}$ using a continuous-stirred tank reactor as explained in a previous publication [1]. The forced hydrolysis process results in a colloidal suspension of anatase nanoparticles, which are centrifuged and washed in deionized water 4 times and once in anhydrous ethanol before being dried overnight at $80^{\circ} \mathrm{C}$ and ground manually to powder form. Acetylene black (Sigma Aldrich) was used as a conductive additive. For standard electrodes PVDF (HSV 900, Arkema) was used as binder, with NMP (Sigma Aldrich) as solvent. Aluminum foil was used as the current collector for all electrodes. Substrates were first pre- treated with $1 \mathrm{M}$ $\mathrm{NaOH}$ solution to improve adhesion of pastes and prevent delamination.

Electrodes fabricated with standard methods had a compositional weight ratio of 80:10:10 anatase to carbon to PVDF. A sol of PVDF mixed with excess n-methyl 2-pyrrolidone ( $\mathrm{nmp}$ ) was mixed with anatase and carbon black. The paste mixture was doctor bladed onto Aluminum foil and dried at $120^{\circ} \mathrm{C}$ for 24 hours. 3-D electrodes were fabricated using a chemo-mechanical method described previously [3]. Anatase and carbon are dry mixed at a ratio $90: 10$ by weight. $140 \mathrm{ml}$ of ethanol is slowly added to the powder mixture stirring constantly. The solution is allowed to stir for 2 minutes before pulse ultrasonication for 2 minutes ( 2 seconds work and 2 seconds rest intervals). The solution returns to stirring and $\alpha$-terpineol is slowly added, before the solution is ultrasonicated again for 2 minutes. The 2 minute stir/ 2 minute sonication cycle is repeated 3 times. Ethyl cellulose is slowly added to the stirring solution at an anatase to ethyl cellulose ratio of $2: 1$ by weight. Another 3 cycles of stirring and sonication are performed before stirring for 5 minutes. The ideal paste viscosity is then reached by evaporating ethanol in a rotary evaporator at $45^{\circ} \mathrm{C}$ and $0.26 \mathrm{~atm}$. The paste is then doctor bladed or screen printed on aluminum foil substrate and dried overnight at $80^{\circ} \mathrm{C}$. The dried film is then sintered at $450{ }^{\circ} \mathrm{C}$ 
following a specific heating profile described in detail in Figure F.7.

A Philips CM200 Transmission Electron Microscope (TEM) was used at 200 $\mathrm{kV}$ to determine particle morphology and crystal structure. TEM specimens were prepared by depositing particles on a copper grid. Brunaer-Emmett-Teller (BET) surface area analysis was performed using a Micromeritics TriStar 3000 apparatus to determine surface area, particle size, and pore size distribution. SEM micrographs were taken using a Hitachi SU-8230 cold-field emission scanning electron microscope (CFE-SEM) (Hitachi High Technologies, Rexdale, Canada) providing high brightness and stable current due to the auto-flash technology. The accelerating voltage was $4 \mathrm{kV}$ and the deceleration mode was used by applying a bias of $3.5 \mathrm{kV}$ leading to a final landing voltage of $0.5 \mathrm{kV}$. The working distance was between 1 and $3 \mathrm{~mm}$. Two through-the-lens detectors were used for secondary (SE) and backscatter (BSE) electron imaging. A filtering grid was used with the top detector in some images to remove SEs and low energy BSEs from the total signal reaching the top detector and was labelled $F x, x$ being the filtering grid bias value (percentage of the deceleration voltage). X-ray mapping was done using a four segments annular retractable Bruker Flat Quad detector located on top of the specimen with a sample surface to Flat Quad detector distance of 1 to $2 \mathrm{~mm}$ providing a solid angle of $1.2 \mathrm{sr}$. The accelerating voltage was $2 \mathrm{kV}$ and the working distance $10 \mathrm{~mm}$.

Compositional electron micrographs were processed to highlight important features. The images were first given values for black-to-white thresholding, and a histogram was produced. This histogram was assigned to a black-to-white gradient. Using ImageMagick software and command prompt command convert a gradient is built as follows:

convert -size $1 \times 33$ gradient:wheat-brown gradient:Brown-LawnGreen $\backslash$
gradient:DodgerBlue-Navy -append gradient_image.png
convert gray_image.jpg $\backslash($ gradient_image.png -flip $\backslash)$-clut $\backslash$
color_image.jpg

First a gradient size 1 pixel by 33 pixels with gradients from wheat to brown, blue 
to lawn green, and dodger blue to navy are built into the image GradientImage.png. Then, using the gradient image, a grayscale image is converted into a new image with color highlighting. This is seen in a variety of images throughout the paper.

Electrochemical characterization was performed using Swagelok-type cells with a BioLogic VSP potentiostat/galvanostat/EIS system. Cells were constructed in an argon-filled glovebox using the titania electrodes as working electrodes and lithium foil as the counter electrode. The electrolyte used was $1 \mathrm{M} \mathrm{LiPF}_{6}$ in EC:DMC:DEC (2:2:1 by weight) mixed solvent (BASF). Galvanostatic charge and discharge was performed between 1 and $3 \mathrm{~V}$ for 100 cycles at both $168 \mathrm{mAg}^{-1}$ and $1680 \mathrm{~mA} \mathrm{~g}^{-1}$, representing $1 \mathrm{C}$ and $10 \mathrm{C}$ rates respectively. Rate capability with equal charge and discharge currents was performed at $\mathrm{C} / 5, \mathrm{C} / 2,1 \mathrm{C}, 2 \mathrm{C}, 5 \mathrm{C}, 10 \mathrm{C}$, and $20 \mathrm{C}$ rate. For all cells, discharge was performed first to insert lithium into pristine $\mathrm{TiO}_{2}$.

\section{F.4 References}

[1] M. J. Sussman, A. Yasin, G. P. Demopoulos, J. Power Sources (Submitted).

[2] K.-E. Lee, C. Charbonneau, G. Shan, G. P. Demopoulos, R. Gauvin, JOM, 61, 52 (2009).

[3] M. J. Sussman, N. Brodusch, R. Gauvin, G. P. Demopoulos, J. Electrochem. Soc., 160(5), A3100 (2013).

[4] S. Ito, P. Chen, P. Comte, M. K. Nazeeruddin, P. Liska, P. Pechy and M Gratzel, Prog. Photovolt.: Res. Appl., 15, 603 (2007). 